Der Medizinischen Fakultät der Universität Göttingen eingereicht von Prof. Dr. Monika Reuss-Borst

\title{
Vergleich verschiedener Messmethoden zur Bestimmung der körperlichen Leistungsfähigkeit und Körperzusammensetzung im Rahmen der „KOLIBRI-Studie“
}

\author{
INAUGURAL-DISSERTATION \\ zur Erlangung des Doktorgrades \\ der Medizinischen Fakultät der \\ Georg-August-Universität zu Göttingen
}

vorgelegt von

Fabian Tobias Joos

aus

Bietigheim-Bissingen

Göttingen 2019 
Die vorliegende Dissertation wurde in Göttingen und Ludwigsburg im Zeitraum von Januar 2016 bis Juli 2018 unter der Betreuung von Prof. Dr. Monika Reuss-Borst (Rehabilitations- \& Präventionszentrum Bad Bocklet) angefertigt.

Dekan:

\section{Betreuungsausschuss}

Betreuer/in

Ko-Betreuer/in:

\section{Prüfungskommission}

Referent/in

Ko-Referent/in:

Drittreferent/in:
Prof. Dr. Monika Reuss-Borst

Prof. Dr. rer. nat. H. K. Kroemer

Prof. Dr. Monika Reuss-Borst

PD Dr. Thomas Ellrott

PD Dr. Thomas Ellrott

Prof. Dr. Thomas Meyer 
Hiermit erkläre ich, die Dissertation mit dem Titel "Vergleich verschiedener Messmethoden zur Bestimmung der körperlichen Leistungsfähigkeit und Körperzusammensetzung im Rahmen der „KOLIBRI-Studie“" eigenständig angefertigt und keine anderen als die von mir angegebenen Quellen und Hilfsmittel verwendet zu haben. 


\section{Inhaltsverzeichnis}

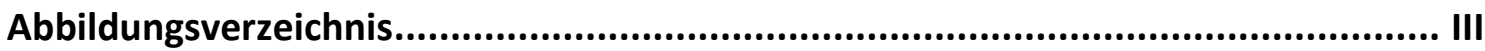

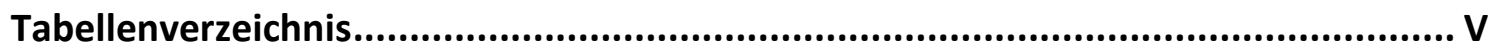

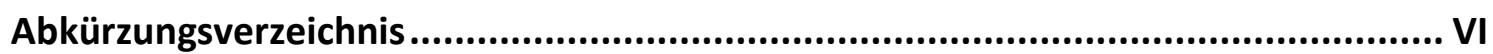

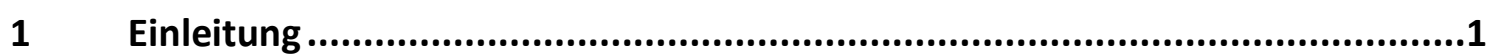

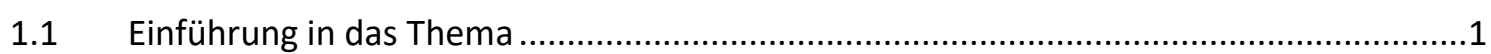

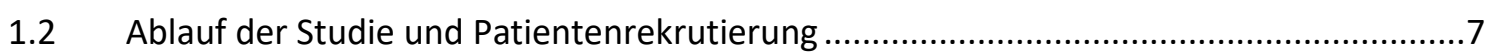

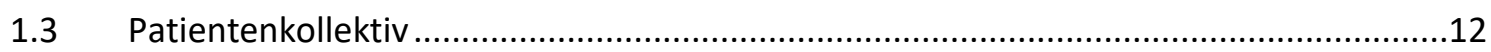

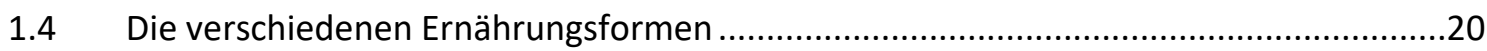

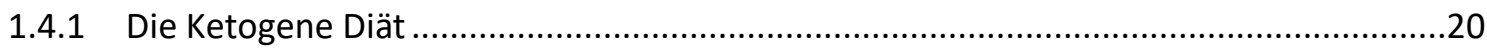

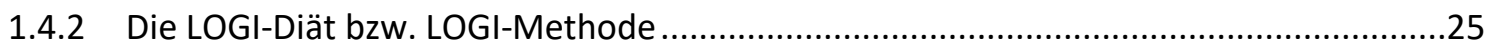

1.4.3 Die Standard-Diät nach den Empfehlungen der Deutschen Gesellschaft für

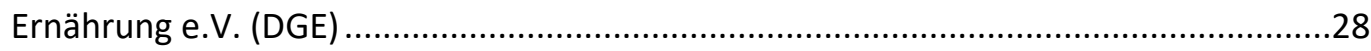

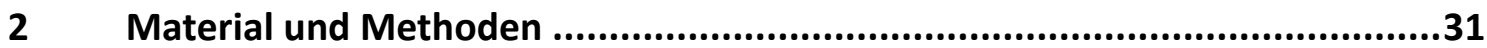

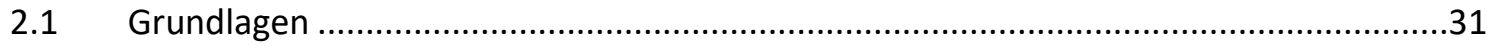

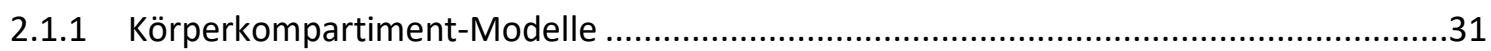

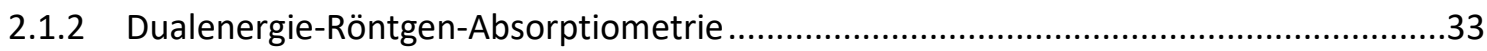

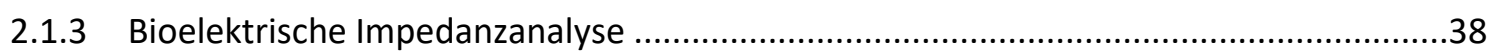

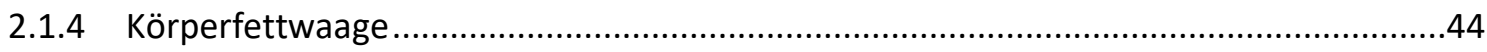

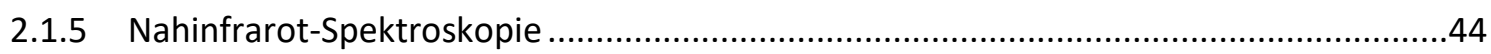

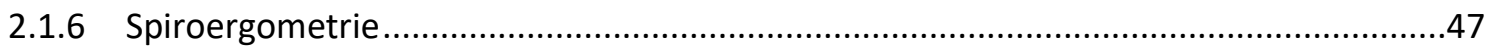

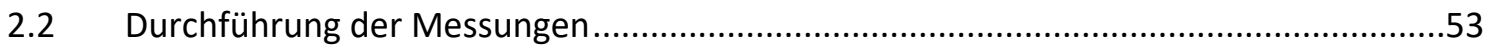

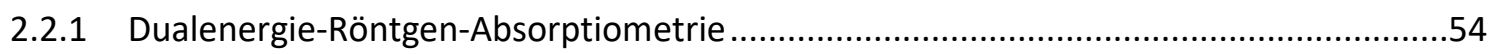

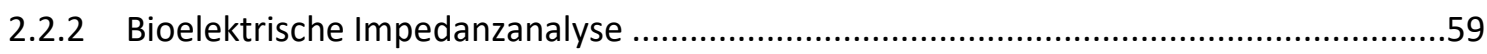

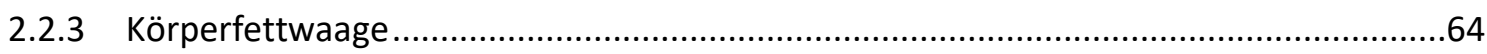

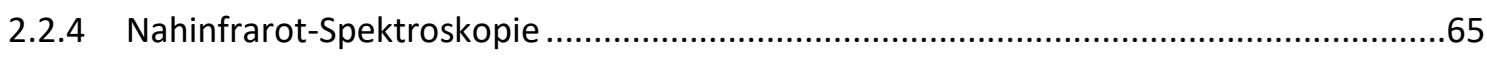

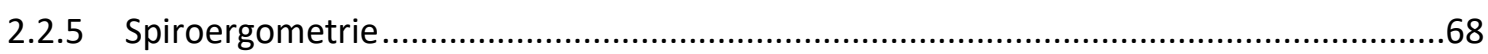

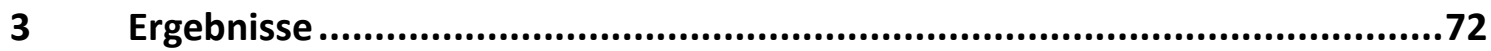

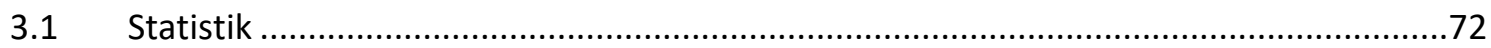

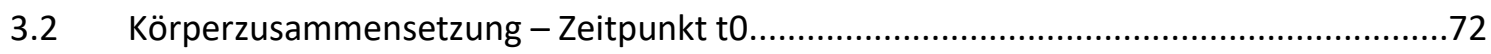

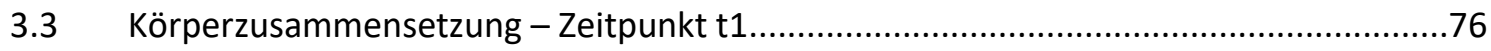

3.4 Körperzusammensetzung - Zeitlicher Verlauf t0 - t1 ...................................................79

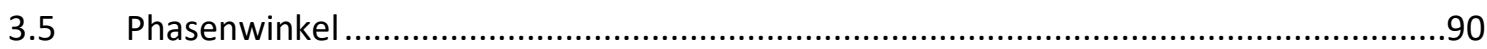

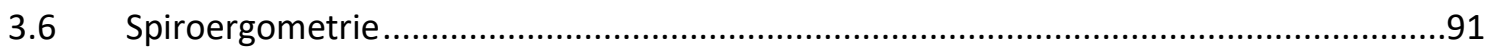




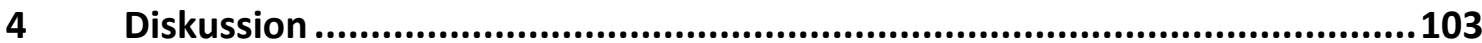

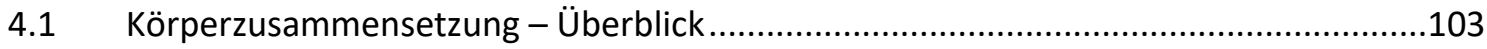

4.2 Körperzusammensetzung - Diskussion der Ergebnisse............................................105

4.3 Körperzusammensetzung - Grundsätze und Einschränkungen der Messmethoden ...111

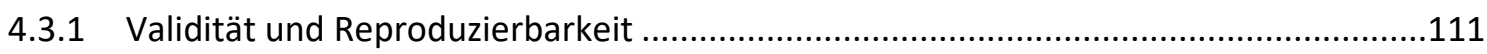

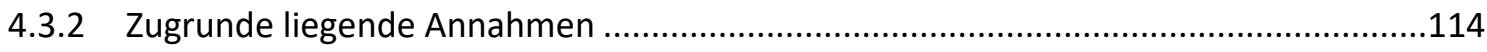

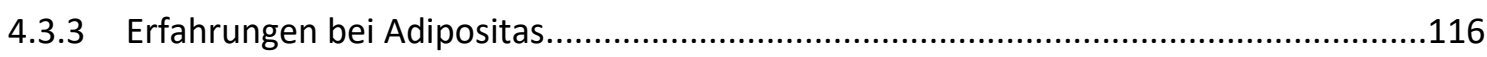

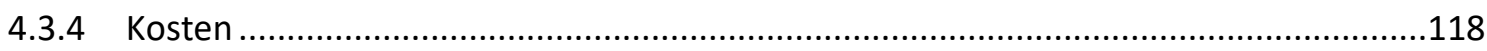

4.4 Körperzusammensetzung - Vergleich der Messmethoden ..........................................119

4.5 Spiroergometrie - Diskussion der Ergebnisse ........................................................123

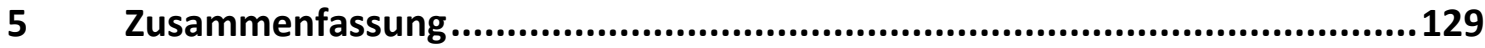

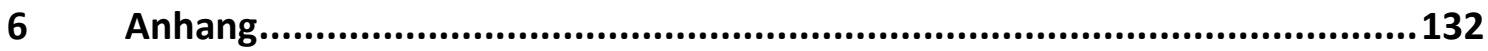

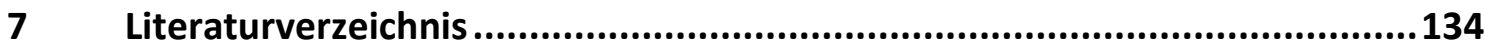

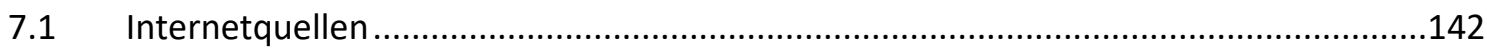




\section{Abbildungsverzeichnis}

Abbildung 1: Altersverteilung im Gesamtkollektiv ....................................................12

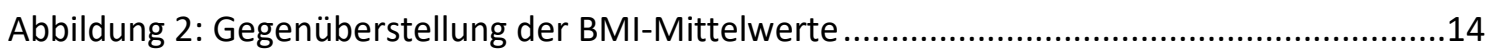

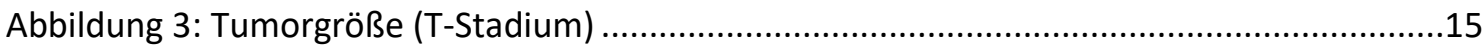

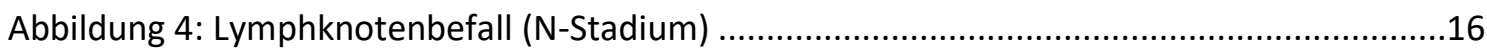

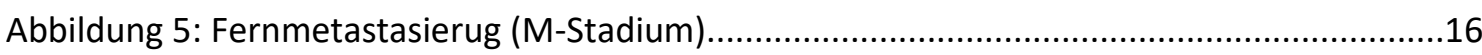

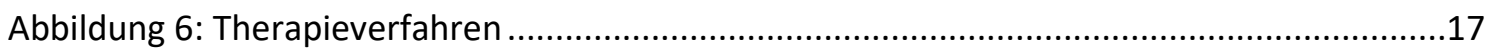

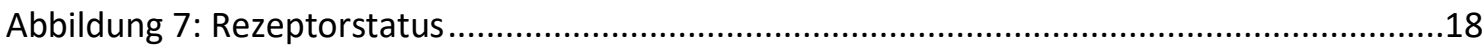

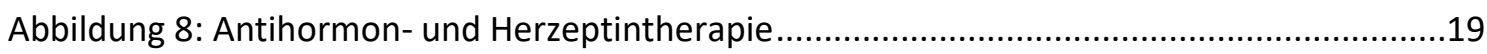

Abbildung 9: Begleiterkrankungen ..............................................................................20

Abbildung 10: Exemplarische Abbildung davon, was alles gegessen werden kann, bis die Kohlenhydratmenge eines einzigen normalen Marmeladenbrötchens erreicht ist .......22

Abbildung 11: Die Original-LOGI-Pyramide nach Dr. Nicolai Worm ....................................27

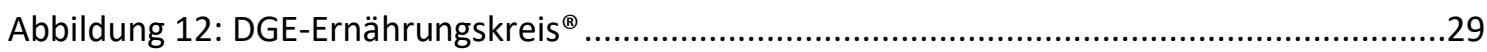

Abbildung 13: Schematische Darstellung der Kompartimentmodelle ...................................31

Abbildung 14: Ausschnitt aus „Modellvorstellung zur Körperzusammensetzung des

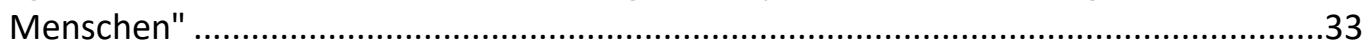

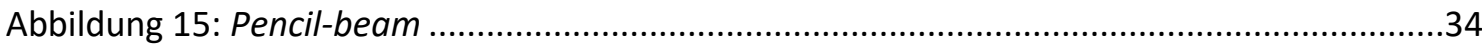

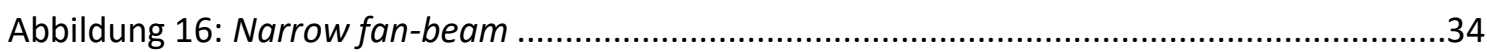

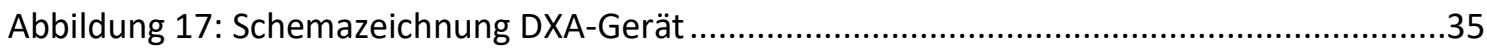

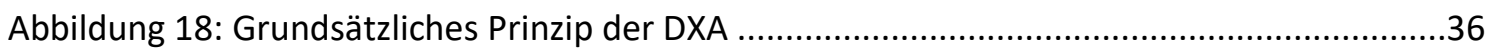

Abbildung 19: Die Bestimmung von $\mathrm{R}$ und $\mathrm{Xc}$ aus Impedanz und Phasenwinkel ......................39

Abbildung 20: Die Entstehung des Phasenwinkels an der Zellmembran - Intakte Zelle..............39

Abbildung 21: Die Entstehung des Phasenwinkels an der Zellmembran - Geschädigte Zelle .....40

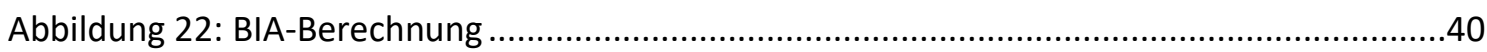

Abbildung 23: Multifrequenzmessung: die Leitungswege verschiedener Frequenzen im

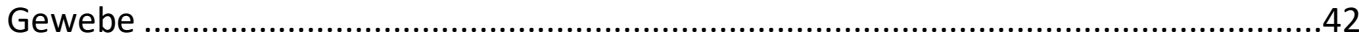

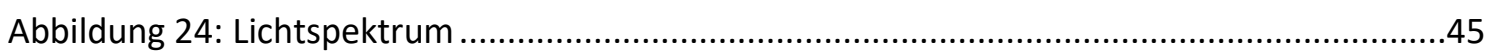

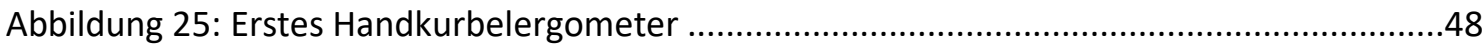

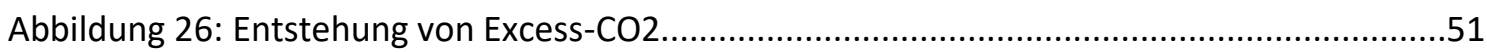

Abbildung 27: In der Studie verwendetes (Original-) DXA-Gerät .....................................55

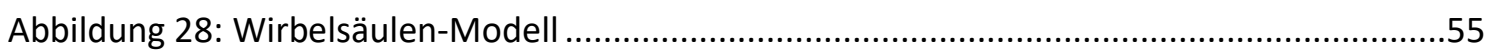

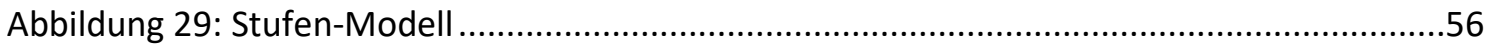

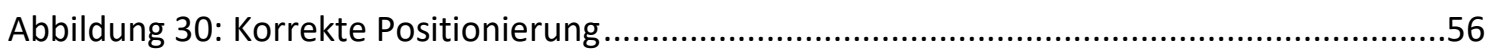

Abbildung 31: Anpassungen im Line- und Point-Mode ...............................................57

Abbildung 32: Ergebnisse für BMC und BMD für die einzelnen Körperregionen ......................58

Abbildung 33: Normbereich der BMD bezogen auf das Alter ............................................58

Abbildung 34: Zusammenfassung aller Messergebnisse (außer BMD) ..................................59

Abbildung 35: In der Studie verwendetes (Original-) BIA-Gerät.........................................59

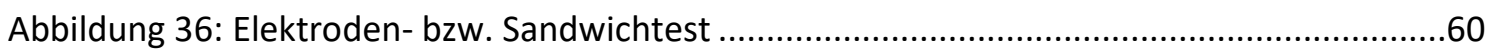

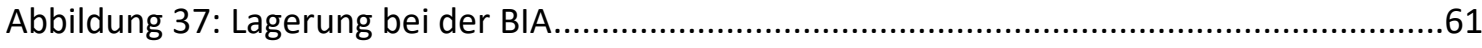


Abbildung 38: Elektrodenpositionierung .......................................................................61

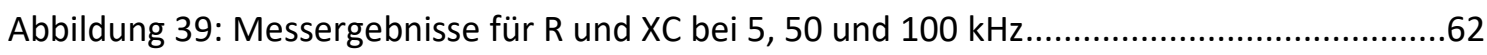

Abbildung 40: Messergebnisse der restlichen Kompartimente............................................63

Abbildung 41: In der Studie verwendete Körperfettwaage ...............................................64

Abbildung 42: Aktivitätsniveau ................................................................................64

Abbildung 43: Berechnete und angezeigte Messergebnisse ................................................65

Abbildung 44: Minimaler- bzw. maximaler Messbereich ....................................................65

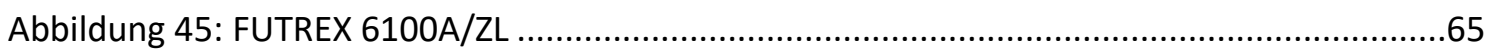

Abbildung 46: Bestimmung des Messpunktes am Biceps................................................66

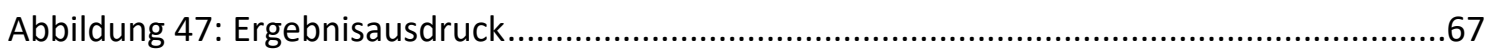

Abbildung 48: Weitere Differenzierung der Kompartimente mittels FUTREX-

Auswertesoftware ................................................................................67

Abbildung 49: Beispielhaftes Schaubild einer kompletten Belastung .....................................71

Abbildung 50: Entsprechende Messergebnisse der Belastung (passend zu Abbildung 49) ........71

Abbildung 51: Mittelwert-Plot - Körpergewicht (t0) ......................................................73

Abbildung 52: Mittelwert-Plot - Fettmasse (t0) ............................................................ 74

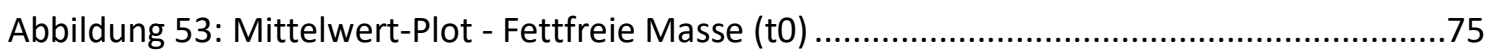

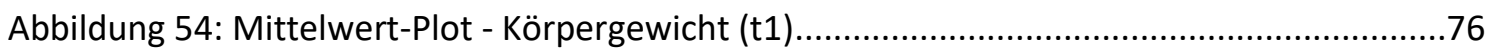

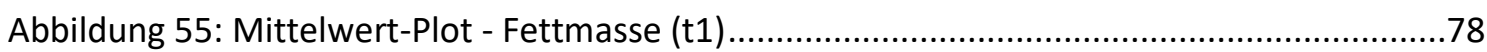

Abbildung 56: Mittelwert-Plot - Fettfreie Masse (t1) ...................................................79

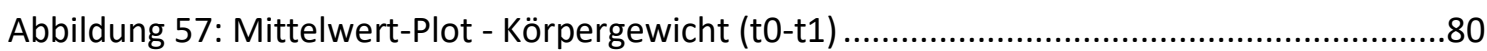

Abbildung 58: Mittelwert-Plot - Körpergewicht KETO (t0-t1) ..........................................81

Abbildung 59: Mittelwert-Plot - Körpergewicht LOGI (t0-t1) ............................................81

Abbildung 60: Mittelwert-Plot - Körpergewicht DGE (t0-t1) .........................................82

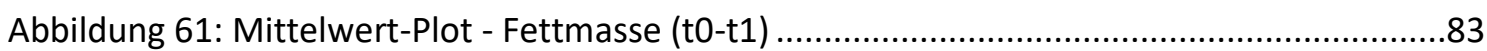

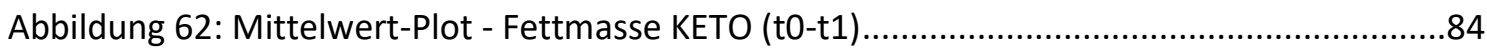

Abbildung 63: Mittelwert-Plot - Fettmasse LOGI (t0-t1) ...................................................85

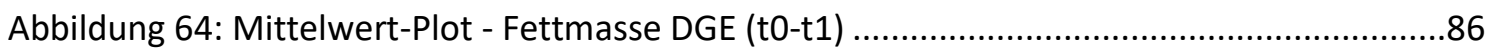

Abbildung 65: Mittelwert-Plot - Fettfreie Masse (t0-t1) ...................................................87

Abbildung 66: Mittelwert-Plot - Fettfreie Masse KETO (t0-t1) ...........................................88

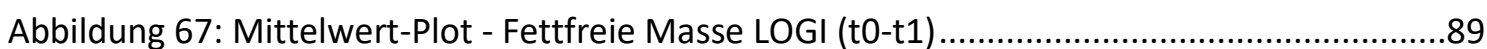

Abbildung 68: Mittelwert-Plot - Fettfreie Masse DGE (t0-t1) ..............................................89

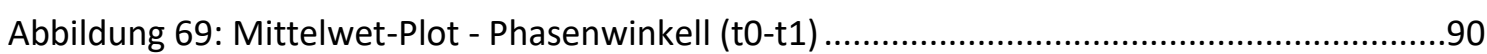

Abbildung 70: Mittelwert-Plot - Ruheumsatzbestimmung ...............................................92

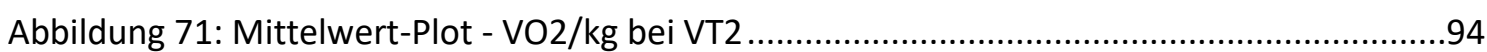

Abbildung 72: Mittelwert-Plot - VO2/kg MAX ............................................................95

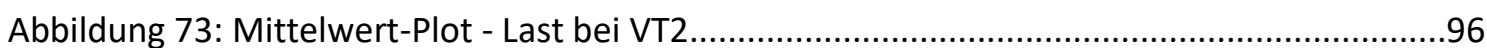

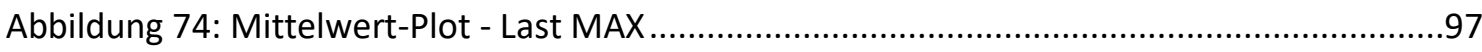

Abbildung 75: Mittelwert-Plot - Zeit bis zum Ende der Belastung........................................100

Abbildung 76: Mittelwert-Plot - Laktatmessung 3 min. nach Belastung .................................101

Abbildung 77: : Interpretation der Tendenzbalken, Anzeigebereich der Messergebnisse und Referenzwerte zu Körperfett und Körperwasser bei der Messung der Körperzusammensetzung mittels Körperfettwaage 


\section{Tabellenverzeichnis}

Tabelle 1: Gewichtsklassifikation Erwachsener anhand des BMI .........................................13

Tabelle 2: Metastasierungsorte ...............................................................................

Tabelle 3: Theoretische R-Werte ausgewählter chemischer Elemente und Verbindungen........37

Tabelle 4: Signifikanzwerte der Post-hoc-Tests bei der Spiroergometrie zum Zeitpunkt t0 .......92

Tabelle 5: Erreichte Fitnesskategorien nach Cooper zum Zeitpunkt t0..................................98

Tabelle 6: Erreichte Fitnesskategorien nach Cooper zum Zeitpunkt t1 .................................98

Tabelle 7: Vergleich der Ergebnisse für LastMAX mit den Sollwerten für die maximale Leistung [Watt] unter Bezug auf das Alter und Körpergewicht nach dem ACSM zum Zeitpunkt t0

Tabelle 8: Vergleich der Ergebnisse für LastMAX mit den Sollwerten für die maximale Leistung [Watt] unter Bezug auf das Alter und Körpergewicht nach dem ACSM zum Zeitpunkt t1

Tabelle 9: Durchschnittliche quantitative Veränderungen der Parameter der Spiroergometrie (t0 - t1)

Tabelle 10: Direkt und indirekt gemessene Zielgrößen der Messmethoden zur Erfassung der Körperzusammensetzung ....

Tabelle 11: Durchschnittliche Differenzen bei der Muskelmasse [kg] t0 - t1 .......................108

Tabelle 12: Durchschnittliche Differenzen beim Körperwasser [l] t0 - t1.............................108

Tabelle 13: Überblick über die Anschaffungskosten bzw. Preisspanne der vier Messgeräte ...118

Tabelle 14: Differenzen der Mittelwerte - FFM (t0)

Tabelle 15: Anteil und Zugehörigkeit zu den jeweiligen Fitnesskategorien derjenigen

Patientinnen, die im Verlauf aus der Studie ausgeschieden waren.

Tabelle 16: Veränderungen der Fitnesskategorien t0 - t1

Tabelle 17: Anteil derjenigen Patientinnen, die im Verlauf aus der Studie ausgeschieden waren und deren Zugehörigkeit zu den Kategorien Sollwert erreicht und Sollwert nicht erreicht

Tabelle 18: Veränderungen bei den Sollwerten t0 - t1

Tabelle 19: Kennzahlen der Verteilung bei der FFM zum Zeitpunkt t0 als Grundlage für die Berechnung der Wilcoxon-Vorzeichen-Rang-Tests. 


\section{Abkürzungsverzeichnis}

\begin{tabular}{|c|c|}
\hline ANOVA & analysis of variance \\
\hline $\mathrm{BCM}$ & body cell mass \\
\hline BIA & Bioelektrische Impedanzanalyse \\
\hline BMI & body mass index \\
\hline $\mathrm{CA}$ & Karzinom \\
\hline COPD & chronic obstructive pulmonary disease \\
\hline $\mathrm{CO} 2$ & Kohlenstoffdioxid \\
\hline $\mathrm{CT}$ & Computertomographie \\
\hline DGE & Deutsche Gesellschaft für Ernährung e.V. \\
\hline $\mathrm{dkfz}$ & Deutsches Krebsforschungszentrum \\
\hline DXA/DEXA & Dualenergie-Röntgen-Absorptiometrie \\
\hline ECM & extracellular mass \\
\hline ECW & extracellular water (extrazelluläres Wasser) \\
\hline FDA & Food and Drug Administration \\
\hline FFM & fat free mass \\
\hline FM & fat mass \\
\hline FUTREX & Nahinfrarot-Spektroskopie-Gerät der Firma FUTREX \\
\hline GI & glykämischer Index \\
\hline GL & glykämische Last \\
\hline HER2 & human epidermal growth factor receptor 2 \\
\hline ICW & intracellular water \\
\hline KETO & Kurzform für ketogene Ernährung/Diät \\
\hline KOLIBRI & Kohlenhydrat-Limitierte-Brustkrebs-Intervention \\
\hline LBM & Lean body mass \\
\hline LOGI & Low Glycemic and Insulinemic Diet (nach Nicolai Worm) \\
\hline NIRS & Nahinfrarot-Spektroskopie \\
\hline NYHA & New York Heart Association \\
\hline $\mathrm{O} 2$ & Sauerstoff \\
\hline RER & respiratory exchange rate/ratio \\
\hline $\mathrm{RQ}$ & respiratorischer Quotient \\
\hline TBW & total body water \\
\hline VCO2 & Kohlenstoffdioxidabgabe \\
\hline VO2 & Sauerstoffaufnahme \\
\hline VT & ventilatory threshold \\
\hline 4C-Model & 4-Kompartimentmodell \\
\hline
\end{tabular}




\section{$1 \quad$ Einleitung}

\subsection{Einführung in das Thema}

Laut Robert Koch-Institut ist Brustkrebs die häufigste Krebserkrankung bei Frauen in Deutschland. 2012 wurden knapp 70.000 Neuerkrankungen registriert (hierzu kamen noch mindestens 5.500 in situ Tumoren), während sich diese Zahl 2004 noch auf etwa 57.000 belief. Die entsprechenden 5-Jahres-Prävalenzen lagen bei 236.000 (2004) bzw. 317.200 (2012).

Das durchschnittliche Erkrankungsalter hat sich über die Jahre nicht wesentlich verändert (2004: 63 Jahre, 2012: 64 Jahre), liegt aber generell deutlich niedriger als bei den meisten anderen Krebserkrankungen.

Trotz gestiegener Inzidenz ist die Anzahl der jährlichen Sterbefälle an Brustkrebs leicht gesunken (Bertz et al. 2010, Robert Koch-Institut 2015). Mit knapp 20\% verursachte die Erkrankung 2012 trotzdem immer noch die meisten krebsbedingten Todesfälle bei Frauen (Becker und Holzmeier).

Die verbesserten Überlebenschancen sind v. a. durch die Möglichkeit der Diagnosestellung zu einem früheren Zeitpunkt der Erkrankung durch konsequente Vorsorge und durch die Fortschritte in der Therapie zu erklären (Robert Koch-Institut 2015). Eine wichtige Rolle spielt dabei auch das zunehmende Wissen über Risikofaktoren und deren Einfluss auf das Brustkrebsrisiko und die Prognose der Erkrankung.

Einige der bekannten Risikofaktoren für die Entstehung von Brustkrebs sind nicht modifizierbar. Hierzu zählen das Alter und die genetische Disposition.

Den Ergebnissen der MARIE-Studie zufolge ist das Risiko an Brustkrebs zu erkranken nicht in jedem Lebensabschnitt gleich hoch. Vor dem 35. Lebensjahr ist zum Beispiel nur eine von 625 Frauen betroffen, während dieses Risiko mit zunehmendem Alter deutlich ansteigt und so schließlich jede 13. Frau bis zum 75. Lebensjahr erkrankt (dkfz 2011).

Mutationen in den sog. Brustkrebsgenen BRCA1 und BRCA2 erhöhen zwar das Brustkrebsrisiko deutlich (beispielsweise senkt sich das durchschnittliche Erkrankungsalter 
dadurch um etwa acht Jahre), sind allerdings nur bei ca. 5 -10\% aller Brustkrebserkrankungen zu finden und damit eher selten (dkfz 2011, Robert Koch-Institut 2015).

Zu den bedingt modifizierbaren Risikofaktoren gehören die sog. Reproduktionsfaktoren, die Hormontherapie in den Wechseljahren und ionisierende Strahlen (Bruhn 2012, dkfz 2011).

Reproduktionsfaktoren, die das Brustkrebsrisiko erhöhen, sind eine frühe Menarche, ein später Beginn der Menopause, Kinderlosigkeit sowie ein höheres Alter bei der Geburt des ersten Kindes. Mehrere bzw. frühe Geburten und Stillen senken hingegen das Brustkrebsrisiko. Insgesamt, so schätzt man, stehen rund 50\% der Brustkrebserkrankungen mit diesen Faktoren in direktem Zusammenhang (dkfz 2011, Robert KochInstitut 2015).

Eine Hormonersatztherapie zur Linderung von Wechseljahresbeschwerden (v. a. bei einer kontinuierlichen Kombinationstherapie aus Östrogenen und Gestagenen über mehr als fünf Jahre) steigert ebenfalls das Brustkrebsrisiko. Dieser Zusammenhang wurde in den Ergebnissen der MARIE-Studie bestätigt (dkfz 2011) und in der Breast Cancer and the Environment-Studie, die im Dezember 2011 auf dem 34. Internationalen Brustkrebs-Symposium vorgestellt wurde, mit großer Evidenz ebenfalls nachgewiesen (Institute of Medicine (U.S.) 2011).

Ionisierende Strahlen, denen Patientinnen beispielsweise bei einer computertomographischen Untersuchung ausgesetzt sind, gehören laut letztgenannter Studie ebenfalls zu den risikosteigernden Faktoren (Institute of Medicine (U.S.) 2011).

Da hier der Handlungsspielraum sehr begrenzt ist, wurde und wird verstärkt nach weiteren Risikofaktoren für die Erkrankung gesucht, die zum einen durch Präventionsmaßnahmen günstig zu beeinflussen sind ( $d k f z$ 2011) und zum anderen aber auch bei bereits bestehender Krebserkrankung durch Modifikation den weiteren Krankheitsverlauf positiv beeinflussen könnten.

Modifizierbare Lebensstilfaktoren sind das Rauchen und Alkoholkonsum, Übergewicht, mangelnde körperliche Aktivität sowie die Ernährung. 
Die Datenlage zum Rauchen ist noch nicht eindeutig. Beim Patientenkollektiv der MARIE-Studie fand sich beispielsweise kein erhöhtes Risiko für Raucherinnen (dkfz 2011). Das Institute of Medicine (U.S.) (2011) führt in seiner Studie an, dass es zum einen groß-angelegte Untersuchungen gab, die nur einen begrenzten Zusammenhang des Rauchens mit dem Brustkrebsrisiko feststellen konnten, zum anderen aber auch bedeutende und gleichzeitig zuverlässige Berichte zu finden sind, die eindeutig zu dem Schluss gelangen, dass das Rauchen kausal mit dem Brustkrebsrisiko zusammenhängt. Zu diesem Ergebnis kamen auch Dossus et al. (2014). Bei ihrem Patientenkollektiv war sowohl aktives als auch passives Rauchen mit einem erhöhten Brustkrebsrisiko verbunden.

Für Alkoholkonsum, Übergewicht und körperliche Aktivität gibt es dagegen eindeutigere Ergebnisse.

In vielen Studien konnte zwischenzeitlich eine klare Assoziation von Alkoholkonsum und einem erhöhten Brustkrebsrisiko nachgewiesen werden. Dabei steigt das Brustkrebsrisiko bereits bei moderatem Alkoholkonsum um $30-50 \%$ (McDonald et al. 2013). Romieu et al. (2015) konnten bei ihrem Kollektiv aus der EPIC-Kohorte sowohl einen positiven Zusammenhang von Alkoholkonsum und Brustkrebsrisiko bei prä- und postmenopausalen Frauen bestätigen als auch eine signifikante Dosis-WirkungsBeziehung $(p<0,01)$ aufzeigen.

Bezüglich des Einflusses von Alkohol auf die Prognose einer bereits bestehenden Brustkrebs-Erkrankung ist die Datenlage noch nicht eindeutig. „Die Mehrzahl der Beobachtungsstudien zeigte keinen Zusammenhang zwischen Alkohol und Prognose“, sodass hier definitiv noch Klärungsbedarf herrscht (Reuss-Borst 2011, S. 1013).

Aus Beobachtungsstudien ist schon lange bekannt, dass Übergewicht einen Risikofaktor für postmenopausalen Brustkrebs darstellt (dkfz 2013). Eine starke Gewichtszunahme lässt laut Emaus et al. (2014) das Brustkrebsrisiko sogar bereits im mittleren Erwachsenenalter (40. - 50. Lebensjahr) ansteigen.

Einige Studien weisen zudem eindeutig darauf hin, dass Übergewicht sowohl vor als auch nach der Diagnose Brustkrebs mit einer schlechteren Krankheitsprognose assoziiert ist. So wurden für prä- und postmenopausale Patientinnen höhere Rezidivraten und ein bis zu 30\% höheres Mortalitätsrisiko beschrieben (Reuss-Borst 2011). 
Übergewicht und Adipositas können durch einen vermehrten Energieverbrauch, d. $h$. Steigerung der körperlichen Aktivität günstig beeinflusst werden. Unabhängig vom Krebsrisiko sinkt dadurch auch das Risiko für weitere Erkrankungen wie beispielsweise Herz-Kreislauf-Erkrankungen, Schlaganfall, Diabetes mellitus oder Osteoporose (dkfz 2016b).

Der Zusammenhang zwischen körperlicher Aktivität und dem Brustkrebsrisiko wurde laut National Cancer Institute (2009) in mehr als 60 weltweit publizierten Studien umfangreich untersucht. Obwohl die prozentuale Risikoreduktion zwischen den Studienergebnissen stark schwankt, weisen die Ergebnisse insgesamt darauf hin, dass körperliche Aktivität sowohl bei prä- als auch postmenopausalen Frauen zu einer Reduktion des Brustkrebsrisikos führt. Die Evidenz für die Intensität und Häufigkeit des zu empfehlenden Trainings ist begrenzt und deshalb noch nicht klar definiert. Laut National Cancer Institute (2009) deuten die meisten Studienergebnisse allerdings darauf hin, dass tägliche moderate bis sehr intensive körperliche Aktivität von 30 - 60 Minuten Dauer mit einer Risikoreduktion für Brustkrebs assoziiert ist und stimmt damit mit den Empfehlungen der WHO von 2010 überein. Auch die WHO ist davon überzeugt, dass dieses Minimum an täglicher körperlicher Aktivität erreicht werden muss, um das Brustkrebsrisiko signifikant senken zu können. Körperliche Aktivität hat außerdem zahlreiche andere pleiotrope Wirkungen. Sie steigert die körperliche Belastbarkeit, erhöht die Lebensqualität und bessert depressive Symptome sowie eine mögliche Fatigue (dkfz 2016a).

Bei der Ernährung spielt nicht nur die Gesamtkalorienzufuhr als eine weitere Stellschraube der Gewichtsmodifikation, sondern v. a. ihre Zusammensetzung eine bedeutende Rolle, die durchaus noch kontrovers diskutiert wird.

Zusammengefasst scheint sich herauszukristallisieren, dass die Analyse von sog. Ernährungsmustern vermutlich aussagekräftiger ist als nur die Analyse einzelner Nahrungsbestandteile. Bezogen auf die Krankheitsinzidenz scheint eine sog. gesunde Ernährung - beispielsweise in den meisten von Brennan et al. (2010) untersuchten Fall-Kontrollund Kohorten-Studien als reich an pflanzlicher Nahrung, aber eher fett- und fleischarm beschrieben - einen gewissen protektiven Effekt zu haben (Reuss-Borst 2011). 
Ein hoher Gemüseverzehr war auch bei Emaus et al. (2016) mit einem niedrigeren Brustkrebsrisiko (hauptsächlich für Hormonrezeptor-negative Tumoren) assoziiert, Buckland et al. (2013) berichteten, dass ein konsequentes Einhalten einer mediterranen Diät ebenfalls das Brustkrebsrisiko senkt (für postmenopausale Frauen und auch eher für Hormonrezeptor-negative Tumoren) und die Ergebnisse von Romieu et al. (2012) deuten darauf hin, dass eine Diät mit hoher glykämischer Last und hohem Kohlenhydratanteil unter postmenopausalen Frauen positiv mit einem erhöhten Risiko für die Entwicklung von Hormonrezeptor-negativen Tumoren assoziiert ist.

Zur Beantwortung der Frage, ob sich durch eine derartige Kost auch die Krankheitsprognose günstig beeinflussen lässt, gibt es kaum Studien.

Eine für die Prävention als günstig geltende Kost muss nicht zwangsweise auch bei Erkrankung günstig sein.

In den letzten Jahren wurden v. a. in der Grundlagenwissenschaft Studien publiziert die zeigen, dass Tumorzellen einen geänderten Stoffwechsel aufweisen und bevorzugt Glukose verstoffwechseln.

Die ungewöhnliche Eigenschaft von Tumorzellen, ihre Energiegewinnung trotz ausreichendem Sauerstoffangebot zum überwiegenden Teil auf die anaerobe Glykolyse zu beschränken, wurde erstmals 1930 vom deutschen Nobelpreisträger Otto Warburg beobachtet und beschrieben.

Aufgrund des deutlich geringeren Wirkungsgrades im Vergleich zur Energiegewinnung durch Zellatmung, war der funktionelle Nutzen dieses glycolytic switch lange Zeit nicht ersichtlich. Heutzutage ist bekannt, dass der Glukoseabbau in Tumorzellen nicht nur der Energiegewinnung dient, sondern ein Großteil der Zwischenprodukte wiederum als Grundbausteine neuer Zellbestandteile verwendet wird.

Um diesen enormen Glukosebedarf decken zu können, wird in Tumorzellen beispielsweise die Anzahl an Glukosetransportern GLUT1, die für eine gesteigerte insulinunabhängige Glukoseaufnahme in die Zelle sorgen, hochreguliert. Diese gesteigerte Aufnahme und Verwertung von Glukose konnte mittlerweile in vielen menschlichen Tumorzellen mittels Positronen-Emissions-Tomographie nachgewiesen werden (Hanahan und Weinberg 2011, Kämmerer 2010). Dabei wird Patienten/-innen ein radioaktiv markierter Zucker (in den meisten Fällen 18F-Fluordeoxyglukose) verabreicht und des- 
sen Anreicherung im Tumorgewebe sichtbar gemacht (Krause et al. 2007). Die Kombination mit der CT-Bildgebung (FDG-PET/CT) liefert dann zusätzliche anatomische und funktionelle Informationen, um so beispielsweise eine gezielte Strahlentherapieplanung optimieren zu können (Shukla und Kumar 2006).

Im Gegensatz dazu stehen die von Breitkreutz et al. (2005) beschriebenen tumorinduzierten Veränderungen des Gesamtstoffwechsels bei Krebspatienten. In diesem Zusammenhang am bemerkenswertesten ist die Tatsache, dass gesunde Zellen eine erheblich gestörte Glukoseverwertung aufweisen und Fette als bevorzugte Energiequelle verwenden.

All diese Beobachtungen haben mit dazu beigetragen, die über Jahrzehnte als gesund geltende Ernährung (kohlenhydratreich und fettarm), wie sie beispielsweise von der Deutschen Gesellschaft für Ernährung e.V. (DGE) empfohlen wird, bei Krebspatienten zu hinterfragen und über eine sog. metabolisch adaptierte Ernährung nachzudenken. Laut Blauem Ratgeber - Ernährung bei Krebs deuten immer mehr Studien darauf hin, dass eine eher fett- und proteinreiche, aber gleichzeitig kohlenhydratarme Kost einer normalen Kost überlegen sein könnte (Deutsche Krebshilfe und Deutsche Krebsgesellschaft Bonn 2015). Auch Breitkreutz et al. (2005) sind davon überzeugt, dass solch eine Diät bei Krebspatienten mit verändertem Stoffwechsel im Sinne einer gestörten Glukoseverwertung und vermehrter Fettoxidation gut geeignet ist und gleichzeitig den Tumorzellen keinen Vorteil bietet. „Allerdings reichen die Ergebnisse noch nicht für eine allgemeine Empfehlung aus" (Deutsche Krebshilfe und Deutsche Krebsgesellschaft Bonn 2015, S. 35).

Ziel der hier ausgewerteten KOLIBRI („,KOhlenhydrat-LImitierte-BRustkrebs-Intervention“) -Studie war es, unterschiedliche Ernährungsformen bei Brustkrebspatientinnen bezüglich ihrer Verträglichkeit und Akzeptanz sowie deren Auswirkungen auf die Lebensqualität, bestimmte Laborparameter, die Körperzusammensetzung und die körperliche Leistungsfähigkeit zu untersuchen.

Bei den drei Ernährungsformen handelt es sich um die sehr kohlenhydratarme und 
gleichzeitig sehr fettreiche ketogene Diät, um die kohlenhydratreduzierte LOGI ${ }^{1}$-Diät und um die kohlenhydratreiche Standard-Diät nach den Empfehlungen der DGE.

Studienhypothese war, dass eine ketogene Diät - oder zumindest eine LOGI-Diät - der Standard-Diät nach DGE bezüglich der o. g. Studienendpunkte überlegen ist. Ein weiteres wichtiges Anliegen der Studie war es zu untersuchen, ob die drei Kostformen (v. a. die ketogene Diät als Extremform) in der Praxis über einen Zeitraum von 4 Monaten überhaupt umsetzbar sind. Eine Aussage zu Krankheitsverlauf oder Prognose kann angesichts der kurzen Beobachtungsdauer bei insgesamt günstiger Prognose der Brustkrebserkrankung nicht getroffen werden.

Die klinische Relevanz der erwarteten Studienergebnisse liegt darin Daten zu gewinnen, die zukünftig eine bessere Basis für die Empfehlung einer bestimmten Kostform bilden können. Ferner sollte durch diese Machbarkeitsstudie ggf. die Grundlage für ein größeres Projekt in der Versorgungsforschung gelegt werden (Reuss-Borst 2013).

Das Hauptaugenmerk dieser Dissertation liegt einerseits auf der Darstellung und statistischen Auswertung der Ergebnisse der Körperzusammensetzung und der körperlichen Leistungsfähigkeit bei den untersuchten Ernährungsformen.

Andererseits sollen die vier in der Studie zur Erfassung der Körperzusammensetzung verwendeten Messmethoden vergleichend diskutiert und wenn möglich eine davon für zukünftige Untersuchungen als Standard-Messmethode empfohlen werden.

\subsection{Ablauf der Studie und Patientenrekrutierung}

Bei der KOLIBRI-Studie (Studiennummer NCT02092753 unter ClinicalTrials.gov) handelt es sich um eine Beobachtungsstudie (Lebensstilintervention), die über einen Zeitraum von zwei Jahren durchgeführt wurde. Die Rekrutierung der Patientinnen erfolgte im Rahmen einer stationären Rehabilitation an der Rehaklinik Am Kurpark in Bad Kissingen. Im Vorfeld war an Brustzentren, bei Selbsthilfegruppen und beim sozialmedizinischen Dienst der Rentenversicherung als zuständigen Kostenträger der onkologischen Rehabilitation über die Studie informiert worden. Über eine Studienaufnahme entschied letztlich der aufnehmende Arzt / die aufnehmende Ärztin der Rehaklinik auf-

\footnotetext{
${ }^{1}$ Die Marke LOGI sowie die LOGI-Methode sind für die systemed GmbH, 44534 Lünen, geschützt.
} 
grund der Anamneseerhebung und anhand der Einschlusskriterien bei Antritt der Reha-Maßnahme sowie die Bereitschaft zur Studienteilnahme der Patientinnen.

Eingeschlossen wurden alle Brustkrebspatientinnen im Alter von 18 - 70 Jahren, bei denen entweder eine Erst-Reha nach primärer Therapie oder eine Folge-Reha bei persistierender Erkrankung - unabhängig vom Krankheitsstadium - durchgeführt werden sollte. Weitere Einschlusskriterien waren ein Karnowsky-Index $>70$ und die Bereitschaft, sich auf eine der drei Ernährungsformen (KETO, LOGI oder DGE) für mindestens 4 Monate konsequent umzustellen.

Während des Rekrutierungszeitraumes von 20 Monaten wurden 549 Patientinnen mit Brustkrebs zur stationären Rehabilitation aufgenommen. Folgende Gründe reduzierten die Zahl der Studienteilnehmerinnen auf 152:

1) Ausschlusskriterien der Studie: 149 Patientinnen

- Zweitmalignom (Ausschließlich Mamma-CA wurden eingeschlossen)

- Mangelnde Deutschkenntnisse, die zu Problemen beim Folgen des Unterrichts und zu Schwierigkeiten bei der Nutzung der Informationsmaterialien geführt hätten

- Psychiatrische Erkrankungen oder Suchterkrankungen (Mangelnde Einverständnisfähigkeit oder Compliance)

- Schwerwiegende internistische Begleiterkrankungen (Insulinpflichtiger Diabetes, Herzinsuffizienz, Herzinfarkt nicht länger als 6 Monate vor Studienbeginn, Herzrhythmusstörungen, dekompensierte Herzinsuffizienz ab NYHA II, Pankreasinsuffizienz, Schwere Niereninsuffizienz oder Dialysepflicht, schwere Leberinsuffizienz)

- Angeborene Stoffwechselerkrankungen, die mit den Ernährungskonzepten inkompatibel gewesen wären

- Lebenserwartung $<12$ Monate

- Schwere Infektionen

- Schwangerschaft (HCG-Test bei prämenopausalen Frauen zum Ausschluss)

- (gleichzeitige) Teilnahme an anderen Studien

2) Fehlendes Interesse an der Studie: 122 Patientinnen 
3) Organisatorische Probleme: 38 Patientinnen (z.B. fehlende Vereinbarkeit mit dem beruflichen Alltag)

4) Sonstige Gründe: 88 Patientinnen

Vor der Aufteilung der 152 Teilnehmerinnen auf die drei verschiedenen Gruppen erfolgte eine ausführliche neutrale Aufklärung und Schulung über alle drei Ernährungsformen.

Auf eine Randomisierung musste verzichtet werden, weil zum einen das konsequente Umsetzen der Vorgaben eine hohe Identifikation und Compliance erforderte und zum anderen weil viele Patientinnen durch die Medien oder eigene Recherchen bereits über verschiedene Ernährungsformen vorinformiert waren und somit eine gewisse Erwartungshaltung vorlag.

Die Gruppengrößen stellten sich wie folgt dar:

- KETO: 29 Patientinnen

- LOGI: 92 Patientinnen

- DGE: 31 Patientinnen

Die rehabilitative Behandlung während der ersten, dreiwöchigen stationären Phase erfolgte nach dem DRV-Therapie-Standard Brustkrebs (ETM KTL 2007). Dabei handelt es sich um ein multimodales Therapiekonzept auf dem Boden des biopsychosozialen Modells von Krankheit und Gesundheit (Deutsche Rentenversicherung 2009). Neben der professionellen Ernährungsberatung mit praktischer Umsetzung und Anwendung der jeweiligen Ernährungsform in der Lehrküche und weiteren edukativen Beratungsund Lehrangeboten (Vorträge und Informationen zu Therapiestandards Brustkrebs, Rückenschule, Herz-Kreislauf und Sport \& Krebs) standen als weitere Hauptbestandteile v. a. auch psychoonkologische und körperlich-funktionelle Aspekte im Fokus der Rehabilitation.

Im Bereich der Psychoonkologie sind hier beispielweise Gesprächsgruppen, Sozialberatung, autogenes Training, Qi Gong und Ergotherapie zu nennen, körperlich aktiviert und gefordert wurden die Patientinnen im Rahmen der Sport- und Bewegungstherapie. 
Auf die stationäre Phase folgte ein 16-wöchiger Zeitraum, in dem die Patientinnen die gewählte Ernährungsform konsequent im häuslichen Umfeld realisieren mussten.

Den Abschluss bildete schließlich die stationäre Etappenwoche (20. Woche), in der die Abschlusserhebung erfolgte.

Während dieser 20-wöchigen Reha-Phase schieden insgesamt 31 Patientinnen (20,4\%) aus der Studie aus, sodass am Ende noch 121 Patientinnen ausgewertet werden konnten.

Die Gründe für das Ausscheiden aus der Studie wurden folgendermaßen zusammengefasst:

1) Gesundheitliche Probleme / verstorben

\begin{tabular}{|c|c|c|}
\hline KETO & LOGI & DGE \\
\hline 2 Patientinnen & 4 Patientinnen & 1 Patientin \\
\hline
\end{tabular}

2) Fehlende Therapieadhärenz

\begin{tabular}{|c|c|c|}
\hline KETO & LOGI & DGE \\
\hline 4 Patientinnen & 3 Patientinnen & 0 Patientinnen \\
\hline
\end{tabular}

3) Private / familiäre / berufliche Probleme

\begin{tabular}{|c|c|c|}
\hline KETO & LOGI & DGE \\
\hline 1 Patientin & 9 Patientinnen & 4 Patientinnen \\
\hline
\end{tabular}

4) Keine Kostenübernahme der Etappen-Reha

\begin{tabular}{|c|c|c|}
\hline KETO & LOGI & DGE \\
\hline 2 Patientinnen & 0 Patientinnen & 1 Patientin \\
\hline
\end{tabular}

Somit beliefen sich die einzelnen Gruppen am Ende der Reha bzw. bei der Abschlusserhebung auf folgende Größen:

KETO: 20 Patientinnen LOGI: 76 Patientinnen DGE: 25 Patientinnen

Alle eben genannten Zahlen sind auf der folgenden Seite noch einmal übersichtlich als Schaubild dargestellt. 
Potenzielle Anzahl an Patientinnen

für die Aufnahme in die Studie
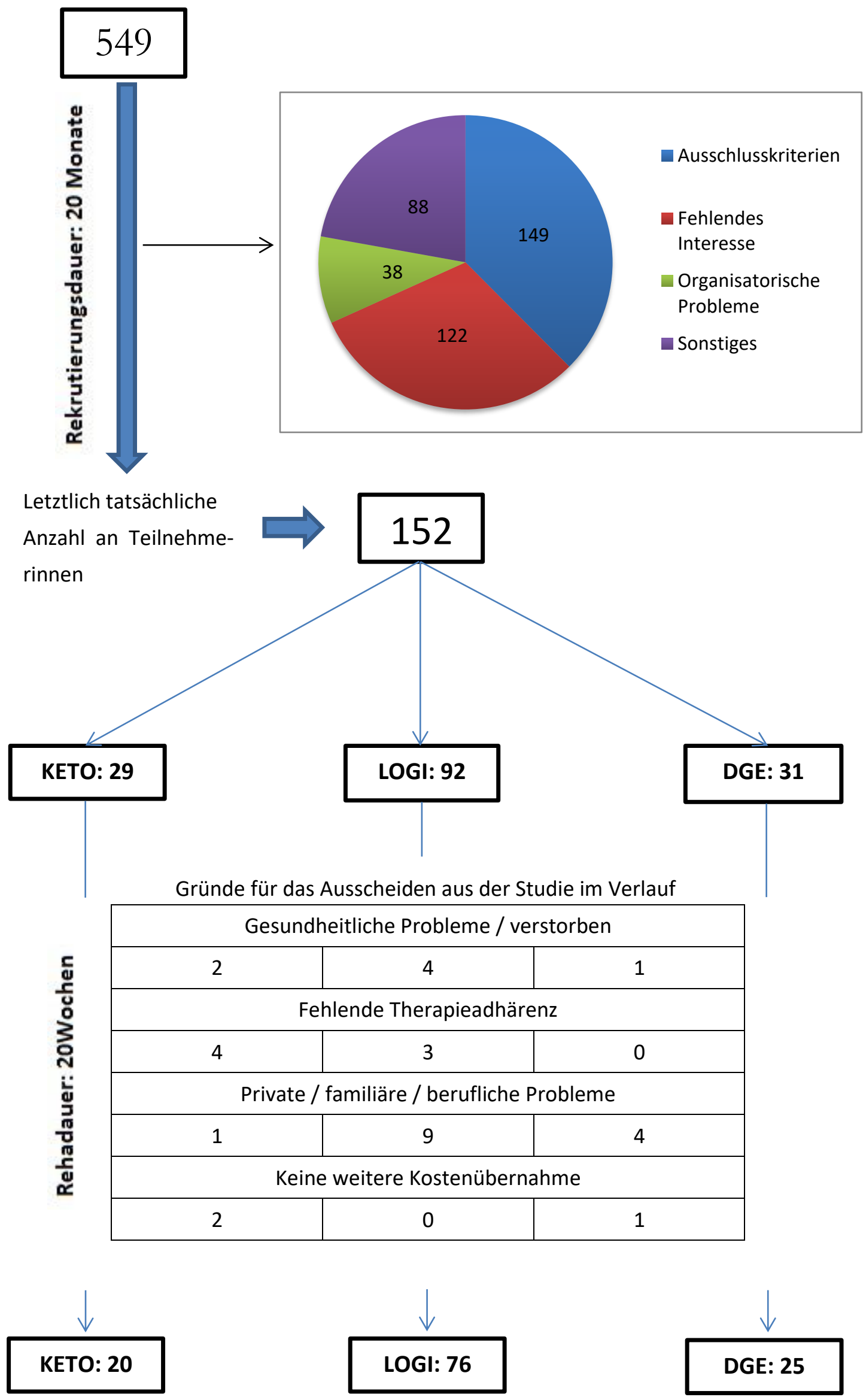

DGE: 25 


\subsection{Patientenkollektiv}

Zum Zeitpunkt t0 (=Rehabilitationsbeginn) wurden bei den 152 Brustkrebspatientinnen soziodemographische Merkmale und klinische Daten erhoben.

Alter

Das durchschnittliche Alter der Patientinnen betrug 51,70 Jahre und unterschied sich in den drei Gruppen fast nicht (KETO = 51,17 Jahre; LOGI = 51,50 Jahre; DGE = 52,77 Jahre). Die jüngste Patientin war 26 Jahre, die Älteste 69 Jahre alt.

84\% der Patientinnen waren jünger als 60 Jahre. Dies spiegelt sich auch in den wenigen Begleiterkrankungen (s. u.) wider.

Einen Überblick über die genaue Altersverteilung im Gesamtkollektiv gibt Abbildung 1.

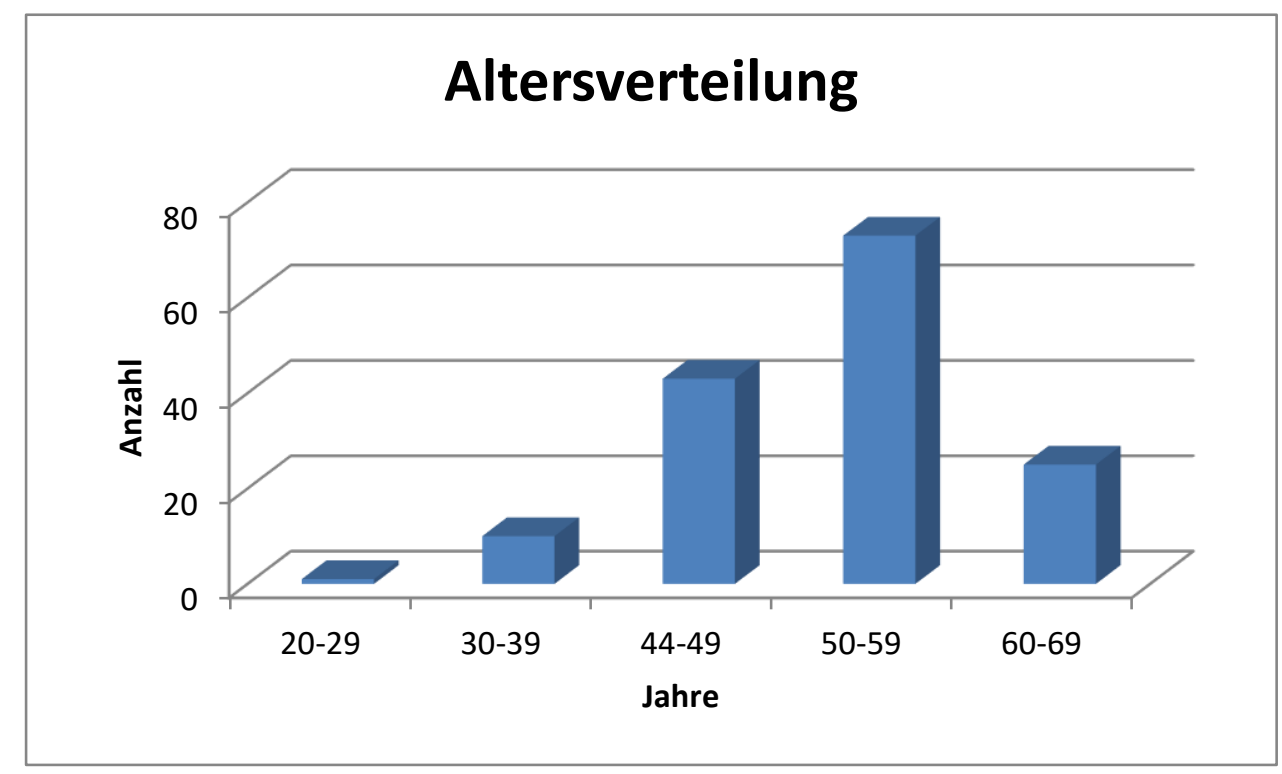

Abbildung 1: Altersverteilung im Gesamtkollektiv

\section{Familienstatus}

Etwa drei Viertel der Patientinnen waren verheiratet bzw. lebten in einer festen Partnerschaft (65,79\% bzw. 7,89\%), während nur etwa ein Viertel aller Patientinnen alleinstehend war (26,32\%). Bei der Verteilung der drei Kategorien verheiratet, in fester Partnerschaft und alleinstehend gab es zwischen den drei Gruppen keine signifikanten Unterschiede $(p=0,989)$. 
Herkunft

Hierbei war auffällig, dass beinahe $90 \%$ der Patientinnen aus der KETO-Gruppe und beinahe 85\% der Patientinnen aus der LOGI-Gruppe* deutscher Herkunft waren, während dieser Prozentsatz in der DGE-Gruppe nur bei knapp 75\% lag.

Insgesamt belief sich der Ausländer-/Migrantenanteil auf 16,67\%.

*von zwei Patientinnen aus der LOGI-Gruppe wurde die Herkunft nicht erhoben, sodass sich alle in diesem Abschnitt angegebenen Prozentwerte auf eine Gesamtzahl von nur 150 Patientinnen beziehen.

\section{Berufstätigkeit}

Zur besseren Übersichtlichkeit werden die sechs erhobenen Kategorien zur Berufstätigkeit hier zu folgenden drei Kategorien zusammengefasst: Berufstätig (Vollzeit/Teilzeit/400€), nicht berufstätig (wie beispielsweise Hausfrauen; inkl. Rentnerinnen) und arbeitslos. Die Verteilung zwischen den drei Gruppen ergab keine signifikanten Unterschiede $(p=0,953)$.

Im Durchschnitt waren mehr als 70\% der Patientinnen berufstätig und davon arbeiteten wiederum mehr als die Hälfte in jeder Gruppe in Vollzeit.

BMI (Body Mass Index)

Laut WHO können anhand des BMI verschiedene Gewichtskategorien unterschieden werden (siehe Tabelle 1).

Tabelle 1: Gewichtsklassifikation Erwachsener anhand des BMI (modifiziert nach: WHO 2000, S. 9. Die Verwendung der Tabelle erfolgt mit freundlicher Genehmigung der World Health Organization)

\begin{tabular}{l|l} 
Kategorie & BMI \\
\hline Untergewicht & $<18,50$ \\
Normalgewicht & $18,50-24,99$ \\
Übergewicht & $>25$ \\
$\quad$ Präadipositas & $25-29,99$ \\
$\quad$ Adipositas Grad I & $30,00-34,99$ \\
Adipositas Grad II & $35,00-39,99$ \\
Adipositas Grad III & $>40$
\end{tabular}


Die BMI-Mittelwerte für jede Gruppe sind in Abbildung 2 dargestellt. Im Gruppenvergleich waren die Patientinnen aus der LOGI- und DGE-Gruppe deutlich übergewichtiger als diejenigen aus der KETO-Gruppe.

Zwischen KETO und LOGI bestand ein signifikanter Gewichtsunterschied $(p<0,01)$, ebenso wie zwischen KETO und DGE $(p=0,048)$. Im Gegensatz dazu war der Unterschied zwischen LOGI und DGE nicht signifikant $(p=0,800)$.

Insgesamt waren 39,5\% der Patientinnen normalgewichtig, d. h. 60,5\% ( $n=92)$ waren demnach übergewichtig bis adipös.

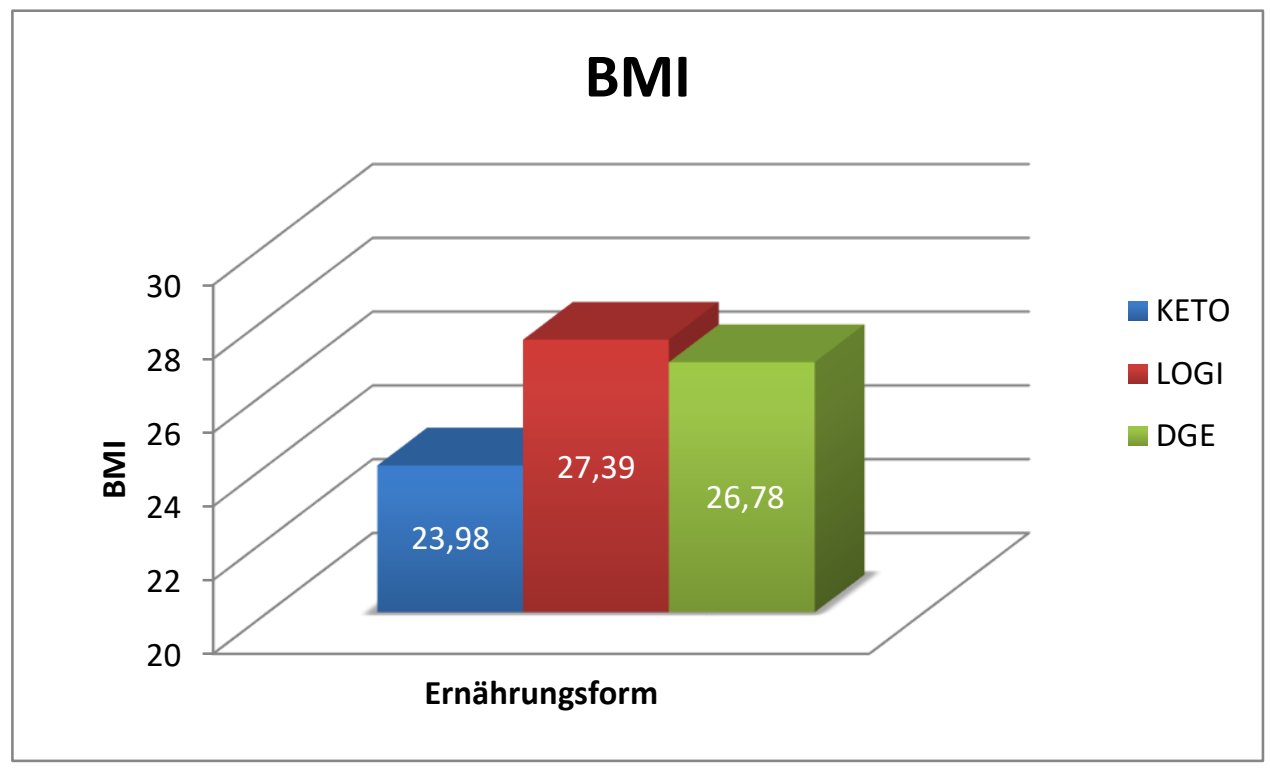

Abbildung 2: Gegenüberstellung der BMI-Mittelwerte

\section{$\underline{\text { TNM - Tumorstadium }}$}

Da bei einer Patientin aus der LOGI-Gruppe keinerlei Angaben zum TNM-Stadium vorlagen, beziehen sich die angegebenen Prozentwerte in dieser Gruppe jeweils auf eine Gesamt-Patientinnen-Anzahl von $\mathrm{n}=91$.

Der deutliche Unterschied beim T0-Stadium (siehe Abbildung 3) zwischen den Gruppen rührt daher, dass bei vier Patientinnen der KETO-Gruppe nur das postoperative TStadium (урт0) bekannt war. Um eine weitere Kategorie und Unübersichtlichkeit zu vermeiden, wurden diese Patientinnen dem Stadium TO zugeordnet. Das initiale präoperative T-Stadium war nicht bekannt. 
In der Verteilung der anderen T-Stadien (T1 - T4) gab es im Gruppenvergleich keine signifikanten Unterschiede $(p=0,278)$.

Insgesamt hatten weniger als $20 \%$ aller Patientinnen ein T-Stadium $\geq$ T3.

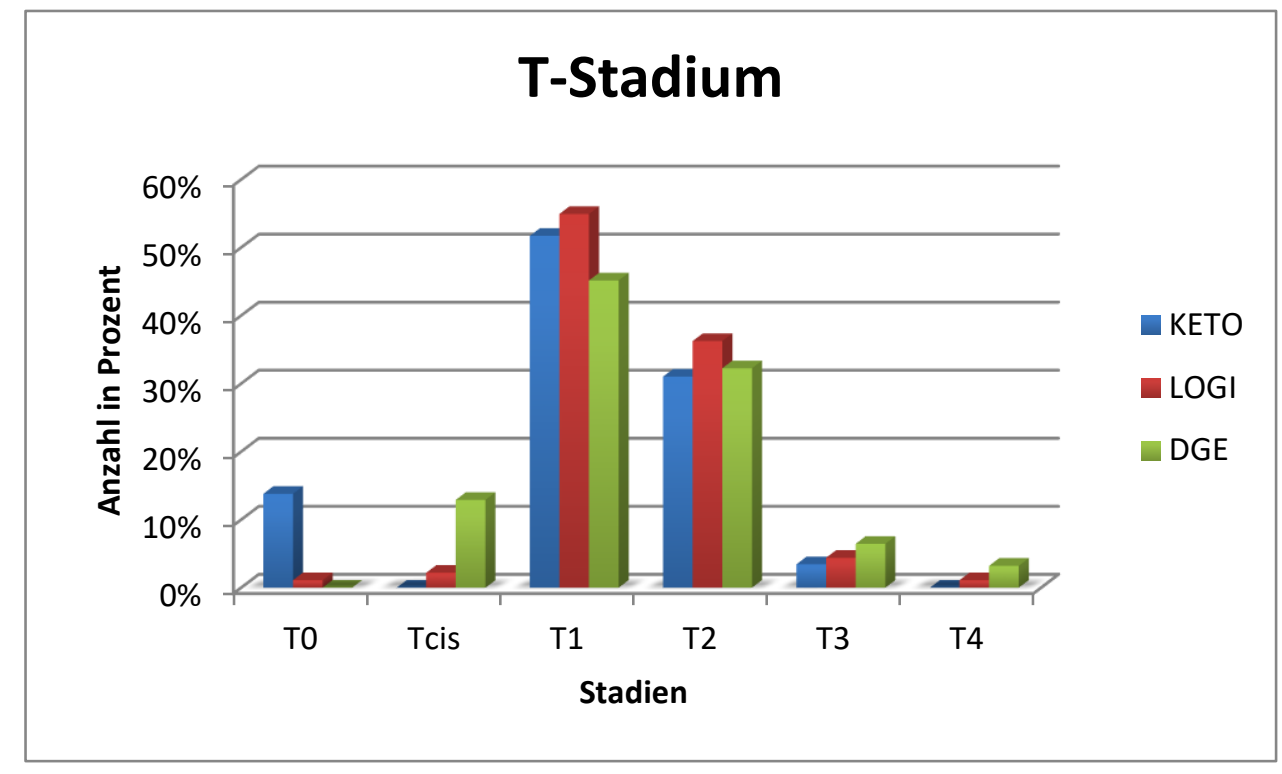

Abbildung 3: Tumorgröße (T-Stadium)

Auch beim N-Stadium (siehe Abbildung 4) gab es zwischen den Gruppen keine signifikanten Unterschiede $(p=0,910)$. Auffällig ist allerdings, dass durchschnittlich $68,3 \%$ aller Patientinnen keinen Lymphknotenbefall aufwiesen (NO) und durchschnittlich nur $18,1 \%$ dem Stadium N1 zugeordnet werden konnten, sodass insgesamt kein ausgeprägter Lymphknotenbefall vorlag.

Bei insgesamt vier Patientinnen (3x KETO und 1x LOGI) war nur das postoperative $\mathrm{N}$ Stadium (ypNO) bekannt. Diese wurden jeweils dem Stadium NO zugeordnet.

Bei einer Patientin der KETO-Gruppe war kein definitives N-Stadium bekannt ( $\mathrm{Nx}=$ Lymphknotenmetastasen vermutet, aber [noch] nicht nachgewiesen).

Im Gruppenvergleich lagen in der KETO-Gruppe deutlich häufiger bereits Fernmetastasen vor als in den anderen beiden Gruppen (siehe Abbildung 5). Der Unterschied zwischen KETO und LOGI war signifikant $(p<0,01)$, zwischen KETO und DGE nicht $(p=0,127)$. Zwischen LOGI und DGE gab es ebenfalls keinen signifikanten Unterschied $(p=0,264)$. Bei insgesamt vier Patientinnen (1x KETO, 2x LOGI, 1x DGE) wurden Fernmetastasen vermutet $(\mathrm{Mx}=$ Fernmetastasen können nicht beurteilt werden - vermutet, aber [noch] nicht nachgewiesen) und bei zwei Patientinnen der KETO-Gruppe wa- 
ren keine Angaben zum M-Stadium bekannt ( $M$ (unbek.)). Am häufigsten von einer Fernmetastasierung betroffen war das Skelettsystem, gefolgt von Lunge und Leber (siehe Tabelle 2).

Tabelle 2: Metastasierungsorte (Sowohl in der KETO- als auch in der LOGI-Gruppe gab es Mehrfachmetastasierungen)

\begin{tabular}{|l|c|c|c|}
\hline Metastasierungsort & KETO $(\mathbf{n}=\mathbf{2 6})$ & LOGI $(\mathbf{n}=\mathbf{9 0})$ & DGE $(\mathbf{n}=\mathbf{3 0})$ \\
\hline Knochen & $19,23 \%$ & $2,22 \%$ & $6,67 \%$ \\
\hline Lunge & $11,54 \%$ & $1,11 \%$ & $0,00 \%$ \\
\hline Leber & $11,54 \%$ & $1,11 \%$ & $0,00 \%$ \\
\hline
\end{tabular}

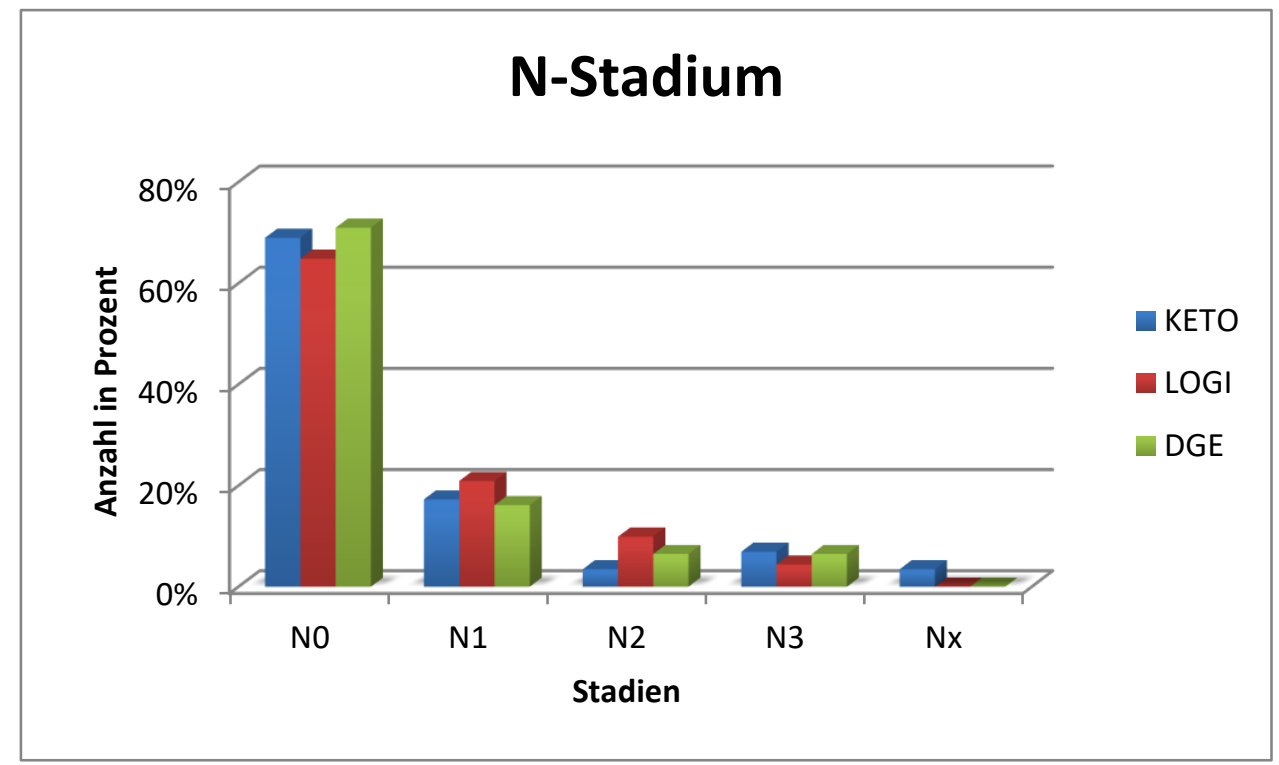

Abbildung 4: Lymphknotenbefall (N-Stadium)

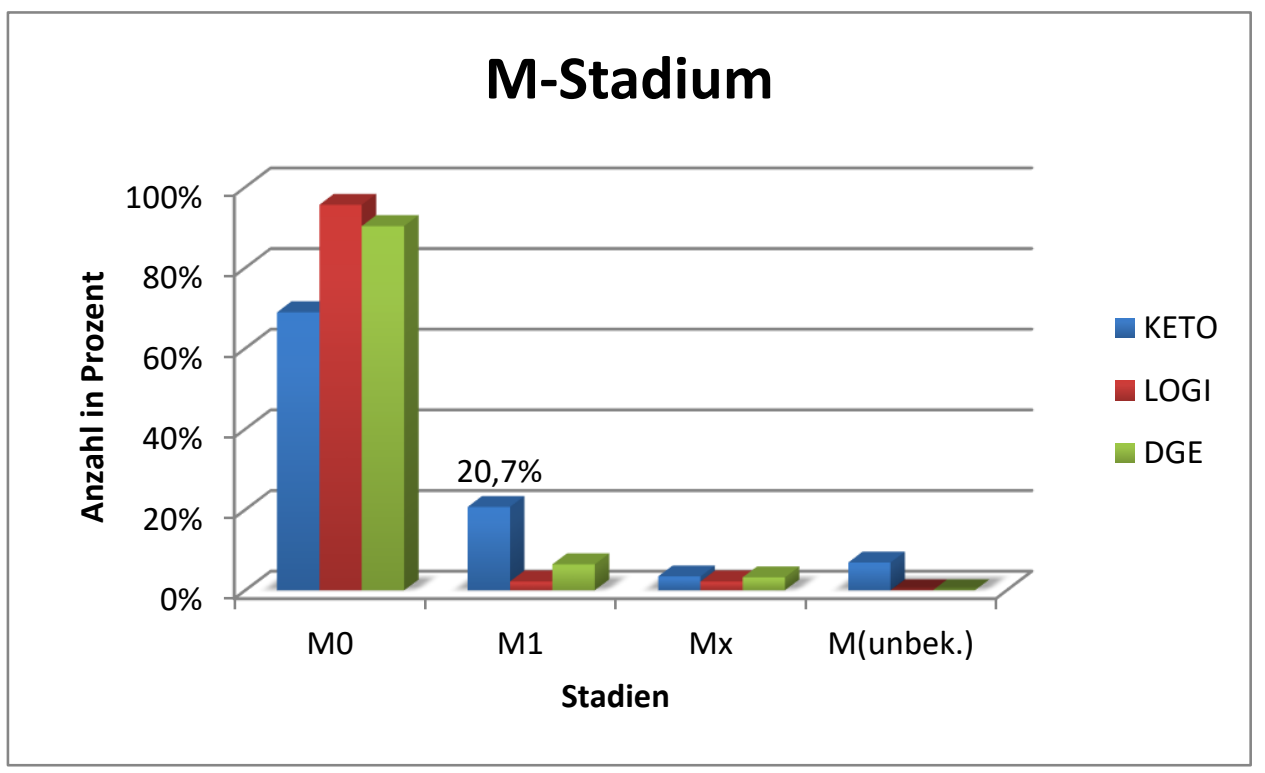

Abbildung 5: Fernmetastasierug (M-Stadium) 


\section{$\underline{\text { Radio- und Chemotherapie }}$}

Im Gruppenvergleich ist zu erkennen, dass insgesamt weniger Patientinnen aus der KETO-Gruppe bestrahlt wurden als Patientinnen aus den anderen beiden Gruppen (siehe Abbildung 6).

Im Gegensatz dazu erhielten die Patientinnen der KETO-Gruppe häufiger eine neoadjuvante Chemotherapie. Die Unterschiede im Gruppenvergleich waren allerdings nicht signifikant ( nneoadj. $=0,276 ;$ padj. $=0,738 ;$ pRT=0,192).

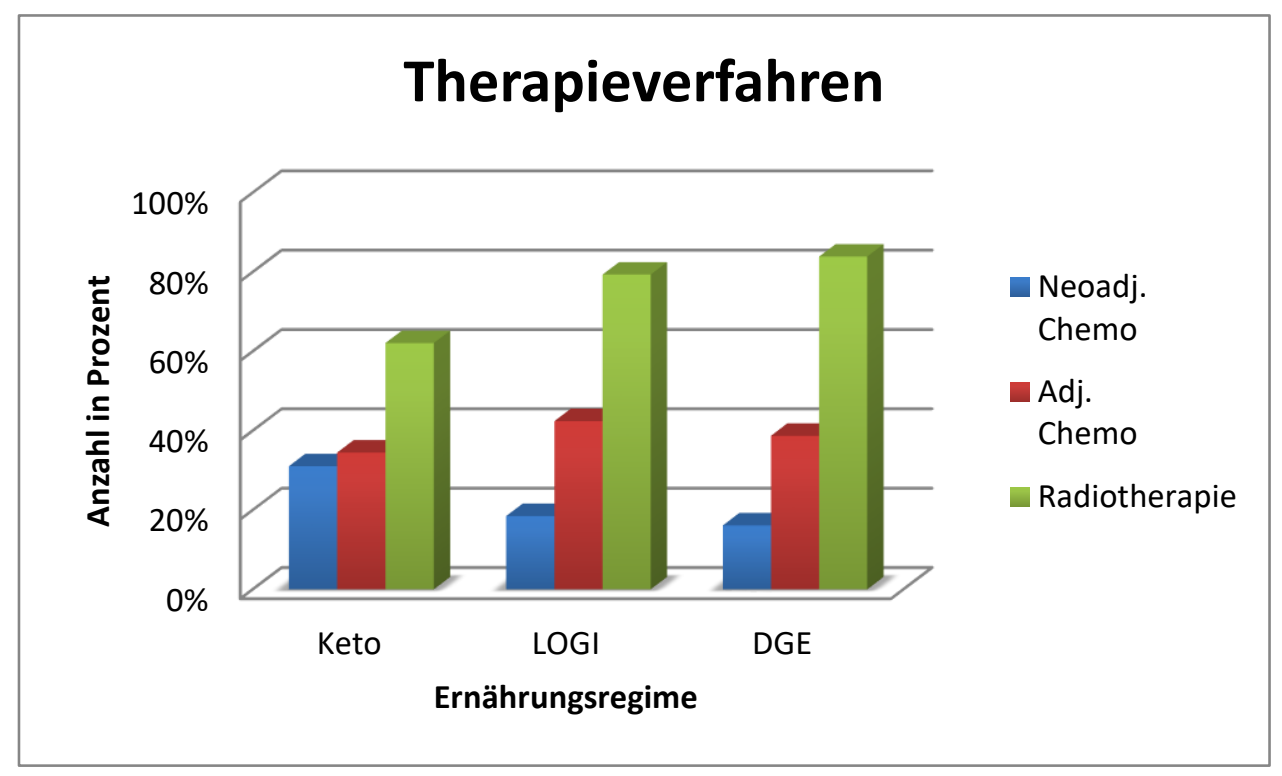

Abbildung 6: Therapieverfahren

\section{Rezeptorstatus und entsprechende Therapien}

Die in Abbildung 7 angegebenen Prozentwerte beziehen sich pro Rezeptor auf die Gesamt-Patientinnen-Anzahl innerhalb jeder Gruppe (bei einer Patientin der KETOGruppe war der Östrogen- und Progesteronrezeptor-Status nicht bekannt).

Zunächst erwähnenswert ist, dass sich die Verteilung pro Rezeptor zwischen den drei Gruppen nicht signifikant unterschied ( $p O ̈ R=0,128 ; p P R=0,112 ; p H E R 2=0,493$ ). Bei der vergleichenden Betrachtung der ersten beiden Rezeptoren fällt auf, dass innerhalb der drei Gruppen jeweils der prozentuale Anteil an Östrogenrezeptor-positiven Patientinnen beinahe gleich groß war wie derjenige an Progesteronrezeptor-positiven Patientinnen.

Im Gegensatz dazu war in allen drei Gruppen jeweils der Anteil an HER2-neu-positiven Patientinnen signifikant geringer als an Östrogen- bzw. Progesteronrezeptor-positiven 
Patientinnen ( $p$-Wert jeweils $<0,01$ ).

Der Anteil an triple-negativen Patientinnen war innerhalb der KETO-Gruppe mit 20,7\% im Vergleich zur LOGI-Gruppe mit 12,0\% $(p=0,236)$ und im Vergleich zur DGE-Gruppe mit 6,5\% $(p=0,140)$ am größten.

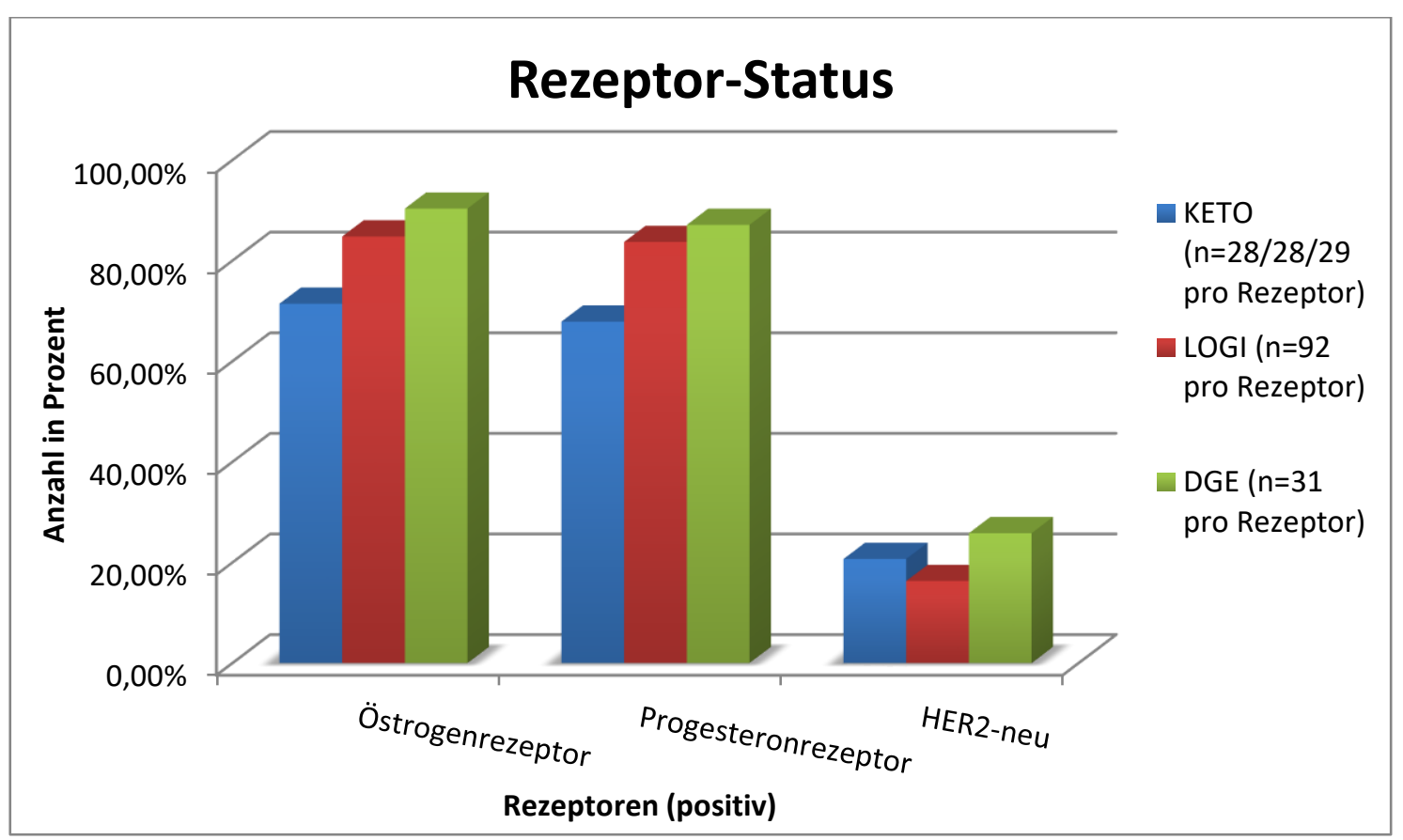

Abbildung 7: Rezeptorstatus

Auffallend bei der Betrachtung der Antihormontherapie (siehe Abbildung 8) im Vergleich zum Östrogen- bzw. Progesteronrezeptor-Status ist, dass nicht alle Patientinnen, bei denen der Primärtumor mindestens einen der beiden Hormonrezeptoren exprimierte, auch entsprechend antihormonell mit Tamoxifen bzw. Aromatasehemmern behandelt wurden.

Innerhalb der KETO-Gruppe war diese Differenz mit 16,26\% (스 4Patientinnen) am größten und innerhalb der LOGI-Gruppe mit 3,26\% (^ 3Patientinnen) am geringsten.

Bei der Herzeptin-Gabe im Vergleich zum HER2-neu-Status gab es ebenfalls Differenzen. Auch hier wurden nicht alle HER2-neu-positiven Patientinnen mit dem entsprechenden Antikörper behandelt. Allerdings waren diese Differenzen hier nicht so ausgeprägt (maximal 6,46\% ( $\triangleq 2$ Patientinnen) innerhalb der DGE-Gruppe). 


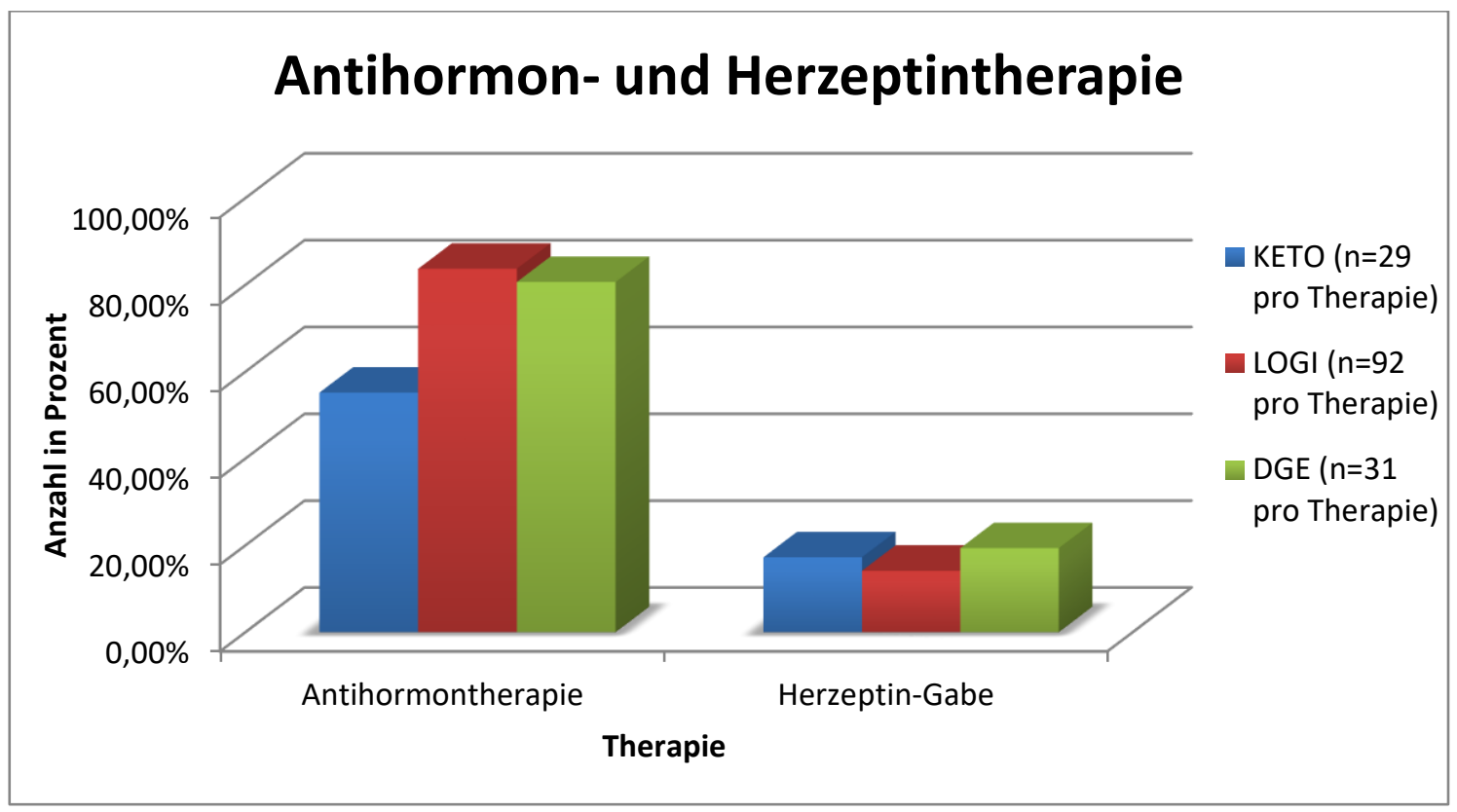

Abbildung 8: Antihormon- und Herzeptintherapie (die hier angegeben Prozentwerte beziehen sich pro Therapie auf die Gesamt-Patientinnen-Anzahl innerhalb jeder Gruppe)

\section{Begleiterkrankungen}

Hier wird nun die anfangs bereits erwähnte Tatsache deutlich, dass die Patientinnen neben der Brustkrebserkrankung an relativ wenigen Begleiterkrankungen gelitten haben.

Erfasst wurden Hypertonie, Diabetes mellitus, periphere Polyneuropathie (pPNP), Hyperurikämie, Arthrose und COPD, wovon in Abbildung 9 nur die drei häufigsten Krankheitsbilder vorgestellt werden.

Mit dem Wissen über die Verteilung des BMI (siehe Abbildung 2: Gegenüberstellung der BMI-Mittelwerte) kann man die Verteilung von Hypertonie und Diabetes mellitus sehr gut damit korrelieren. In der LOGI-Gruppe war der BMI mit durchschnittlich 27,4 am höchsten, dementsprechend gab es in dieser Gruppe insgesamt am meisten Hypertonikerinnen und Diabetikerinnen.

In den anderen beiden Gruppen war der Anteil entsprechend niedriger.

Deutlich zu erkennen ist, dass in jeder Gruppe jede vierte bis fünfte Patientin an einer peripheren Polyneuropathie litt. Ursache hierfür ist sicherlich der Einsatz von Taxanen im Rahmen der Chemotherapie. 
Bei der Verteilung der drei beschriebenen Erkrankungen gab es im Gruppenvergleich keine signifikanten Unterschiede ( $p$ Hypertonie=0,057; pDiabetes=0,413; $p p P N P=0,916$ ).

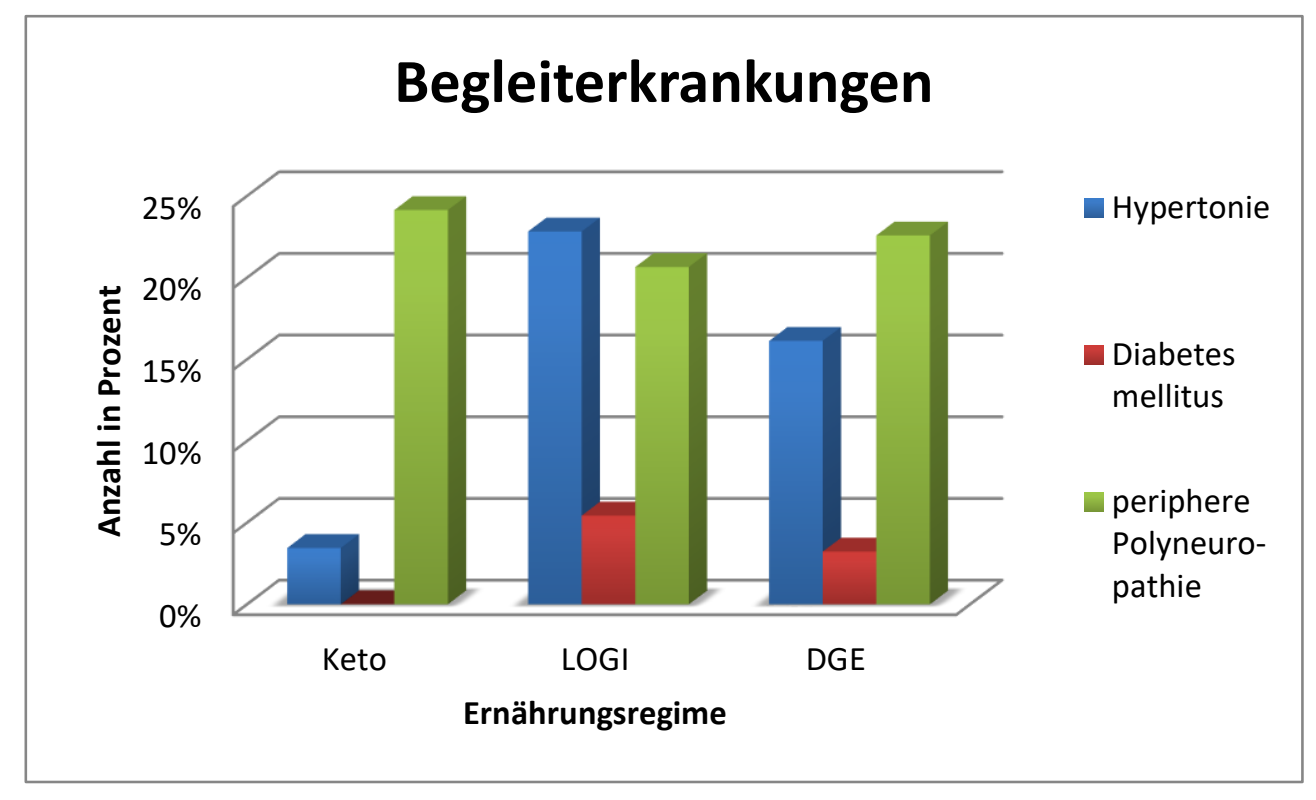

Abbildung 9: Begleiterkrankungen

\subsection{Die verschiedenen Ernährungsformen}

\subsubsection{Die Ketogene Diät}

„Wichtigstes Prinzip der ketogenen Ernährung ist die stark eingeschränkte Zufuhr von Glukose bzw. Kohlenhydraten [...] bei deutlich erhöhter Zufuhr von guten Fetten und ausgeglichener Eiweißbilanz" (Kämmerer 2010, S. 16). Bei dieser Ernährungsform stellt der Körper auf Energiegewinnung aus Fettsäuren und Ketonkörpern um. Die dann noch in kleinen Mengen v. a. für Nervenzellen, Zellen der Nebennieren und Erythrozyten benötigte lebensnotwendige Glukose kann aus den übrigen noch in der Nahrung vorhandenen Kohlenhydraten oder im Rahmen der Gluconeogenese gewonnen werden, sodass der Nüchternblutzucker-Spiegel konstant gehalten wird.

Als physiologische Ketose bezeichnet man einen Zustand im Körper, bei dem es infolge des gesteigerten Fettsäureabbaus zur Produktion von sogenannten Ketonkörpern (Acetoacetat, $\beta$-Hydroxybutyrat und Aceton) in der Leber kommt. Abgesehen von Aceton können diese Ketonkörper von fast allen Zellen des Körpers zur Energiegewinnung 
genutzt werden. Überschüssige Ketonkörper werden mit dem Urin ausgeschieden, wo sie mit Teststreifen nachgewiesen werden können (Kämmerer 2010).

Da Tumorzellen für ihr Wachstum auf enorme Mengen an Kohlenhydraten angewiesen sind und in ihnen oftmals die Fähigkeit zur Fettverbrennung reduziert oder sogar ganz abgeschaltet ist, stellt die ketogene Ernährung hier einen attraktiven Angriffspunkt dar. Gesunde Zellen werden ausreichend mit Energie in Form von Fetten und Eiweißen versorgt, während für Tumorzellen die Bereitstellung von Zuckern als Energiequelle deutlich reduziert wird.

Ein weiterer wichtiger Nachteil, der sich unmittelbar hieraus für die Tumorzellen ergibt ist, dass insgesamt deutlich weniger Insulin ausgeschüttet wird. Da Insulin für viele Tumoren auch ein Wachstumssignal darstellt, bedeuten niedrige Insulinspiegel entsprechend weniger Wachstumsanregung (Kämmerer 2010).

Um sicher den Zustand der Ketose zu erreichen, sollte der Anteil aus Fett an der Gesamtenergie mindestens 75 - $85 \%$ betragen, während sich die verbleibenden 15 - 25\% auf Eiweiß und Kohlenhydrate verteilen (Kämmerer et al. 2012 - 2013).

Hierfür wird der ketogene Quotient (KQ) angesetzt. Ein KQ von 2:1 bedeutet, dass auf $2 \mathrm{~g}$ Fett, $1 \mathrm{~g}$ Eiweiß und Kohlenhydrate kommen. Da mehr Eiweiß verzehrt werden soll, lautet die Faustregel (bezogen auf das Gewicht der Nahrungsmittel):

$2 \mathrm{~g}$ Fett : 0,75 g Eiweiß und 0,25 g Kohlenhydrate

Bei einem geringeren Anteil an Fettenergie ist bei den meisten Menschen eine stabile Ketose nicht zu erreichen; im Falle der Epilepsietherapie wird oft sogar ein KQ von 4:1 oder mehr angestrebt (Baumeister 2004).

Um dies erreichen zu können, müssen kohlenhydrathaltige Sättigungsbeilagen wie beispielsweise Kartoffeln, Reis, Nudeln, Brot und Getreideflocken aus der Ernährung gestrichen bzw. durch kohlenhydratarme Alternativen ersetzt werden. Dies erfordert eine sorgfältige Auswahl der Produkte beim Einkauf, ist allerdings mit normalen, alltäglichen Lebensmitteln möglich (Kämmerer 2010). 
Mit der Abbildung 10 soll gezeigt werden, dass bei einer ketogenen Diät trotz der Restriktionen keine Einbußen beim Genuss befürchtet werden müssen.

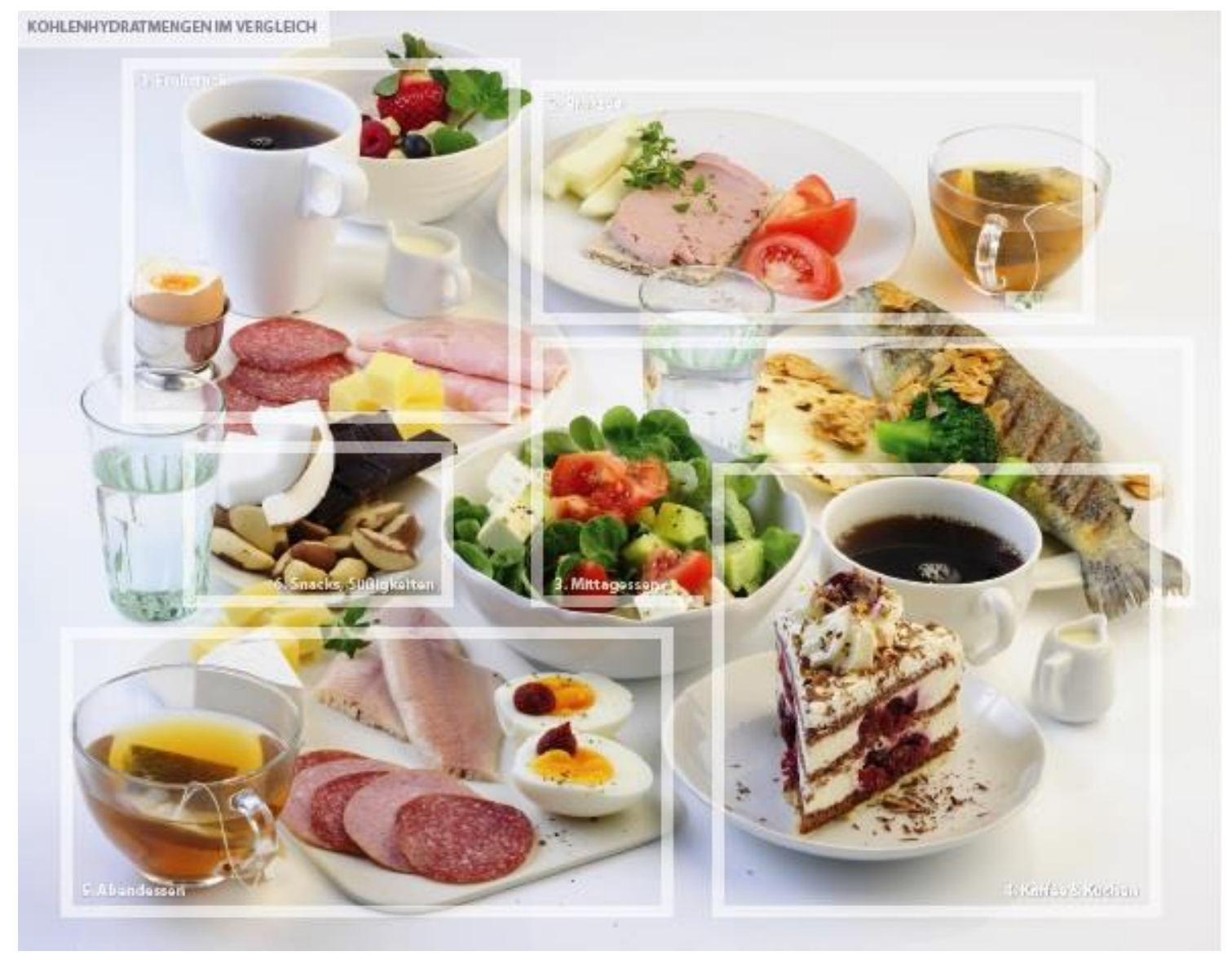

Abbildung 10: Exemplarische Abbildung davon, was alles gegessen werden kann, bis die Kohlenhydratmenge eines einzigen normalen Marmeladenbrötchens erreicht ist (aus Kämmerer et al. 2012 - 2013; Die Verwendung der Abbildung erfolgt mit freundlicher Genehmigung des systemed Verlags)

Um die Bedenken vor zu viel Fett zu reduzieren, soll gleich vorab erwähnt werden, dass viele wissenschaftliche Studien eindeutig belegen, dass Fettkonsum nichts mit den klassischen kardiovaskulären Erkrankungen zu tun hat (Kämmerer 2010). Im Rahmen der ketogenen Diät sollten demnach v. a. fetthaltige Produkte verzehrt werden.

Im Folgenden werden einige der im Rahmen der Ketogenen Diät empfohlenen Nahrungsmittel(gruppen) zusammengefasst dargestellt.

Bei Milchprodukten ist auf eine hohe Fettgehaltsstufe zu achten, bei Käse sind besonders fettreiche Sorten und gut gereifter Bergkäse zu bevorzugen und bei Fisch sind v. a. 
die fetten Kaltwasserfische wie beispielsweise Hering, Makrele und Lachs gut geeignet. Letztere sind sehr reich an Omega-3-Fettsäuren und daher besonders empfehlenswert.

Bei Fleisch- und Wurstwaren sollte so oft wie möglich auf hochwertige Produkte aus Weidehaltung oder aus Haltung mit naturbelassenem Futter zurückgegriffen werden. Auch sie weisen einen hohen Gehalt an Omega-3-Fettsäuren auf, wohingegen bei fast allen Salami- und Wurstsorten Zucker zugesetzt ist (Kämmerer 2010).

Eine weitere reichhaltige Quelle von Omega-3-Fettsäuren bilden hochwertige kaltgepresste Pflanzenöle. Hierzu zählen Hanf-, Leinsamen-, Raps- und Fischöl, die den Körper zusätzlich noch mit wichtigen fettlöslichen Vitaminen versorgen. Wichtig ist bei diesen Ölen darauf zu achten, diese nicht zu erhitzen, da dabei sonst schädliche Produkte entstehen. Zum Braten können beispielsweise ungehärtetes Palm- oder Kokosfett verwendet werden (Kämmerer 2010).

Im Gegensatz dazu sollten Sonnenblumen-, Mais- oder Weizenkeimöle vermieden werden, da diese reich an Omega-6-Fettsäuren (Arachidonsäurevorstufen) sind. Ein günstiges Omega-3- zu Omega-6-Verhältnis vermindert die Produktion von proinflammatorischen Signalmolekülen, wodurch deren begünstigende Wirkung auf die Entstehung und das Wachstum eines Tumors verringert werden kann (Kämmerer et al. 2012 - 2013).

Zur Verfeinerung von z. B. Quark oder Salat eignet sich sehr gut der Einsatz von verschiedenen Nüssen (Walnüsse, Paranüsse; nicht Cashew-Kerne) und Nussflocken. Auch sie enthalten wichtige Omega-3-Fettsäuren und sollten am besten frisch geschrotet verzehrt werden.

Bei Gemüse muss wie bei der LOGI-Diät (s. u.) auf stärkehaltige Produkte wie Karotten, Erbsen, Bohnen oder Linsen aufgrund des daraus resultierenden, starken Blutzuckeranstieges verzichtet werden. Ansonsten stellt grünes Gemüse (dazu zählen u. a. Spinat, Zucchini, Gurke, Kohl und grüne Bohnen) eine ideale Ergänzung zu Fleisch und Fisch dar.

Aufgrund des hohen Glukose- und Fruktosegehalts muss Obst im Rahmen der ketogenen Diät weitgehend vermieden werden. Besonders zu empfehlen - nicht nur auf- 
grund des geringen Kohlenhydrat-Gehalts, sondern wegen zusätzlich krebshemmender Eigenschaften - sind dagegen Beeren aller Art (auch gefroren und ungezuckert).

Wie bei den Empfehlungen der Deutschen Gesellschaft für Ernährung e.V. (s. u.) bleibt auch hier Spielraum für Süßes. Wer z. B. nicht auf Schokolade verzichten möchte, darf sich gelegentlich ein Stück dunkle Schokolade mit hohem Kakaoanteil gönnen. Mittlerweile gibt es aber auch zahlreiche Rezepte für ketogene Kuchen, sodass auch die Kuchenliebhaber auf ihre Kosten kommen.

Zur Vermeidung von Nierensteinen und zur Ausschwemmung überschüssiger Ketonkörper, sollten täglich mindestens 2 - 3 I Flüssigkeit aufgenommen werden. Softdrinks und Bier sind aufgrund des extrem hohen Kohlenhydrat-Anteils zu vermeiden, während Wasser und ungesüßter (Kräuter- oder Grüner-) Tee unbegrenzt getrunken werden können.

Zu erwähnen und zu bedenken ist, dass die ketogene Diät primär nicht zum Abnehmen gedacht ist, sondern eher eine Umstellung des Stoffwechsels bewirken und einer Tumorkachexie entgegenwirken soll. Wer doch eher abnehmen möchte, sollte die Ölbeimengungen reduzieren und die gewohnten Portionen (an Fleisch oder Fisch) beibehalten.

Bei der Ernährungsumstellung auf die ketogene Diät sollte der Kohlenhydratanteil innerhalb der ersten Woche nur schrittweise und nicht zu schnell reduziert werden. Ansonsten drohen Unwohlsein, Kopfschmerzen, Übelkeit und Abgeschlagenheit. Sobald die individuelle Schwelle von Kohlenhydraten erreicht ist, bei der die Ketose eintritt (täglich mittels Urin-Teststäbchen zu kontrollieren), erfordert diese Ernährungsform ein konsequentes Umsetzen der Empfehlungen. Zu lange Unterbrechungen gefährden den Erfolg.

Zusammengefasst erfordert die ketogene Ernährung viel Zeit, sich mit den genauen Ernährungsempfehlungen vertraut zu machen und viel Geduld und Disziplin, alle Nahrungsmittel abzuwiegen und die Kohlenhydratzufuhr mit Hilfe von Nährwerttabellen genau zu berechnen (Kämmerer 2010). 


\subsubsection{Die LOGI-Diät bzw. LOGI-Methode²}

Nach Prof. David Ludwig stand LOGI ursprünglich für Low Glycemic Index. Zusammen mit einer Arbeitsgruppe an der Harvard-Universität entwickelte Ludwig zudem die bekannte LOGI-Pyramide (siehe Abbildung 11). Angestrebtes Ziel dieser Ernährungsform war ein möglichst niedriger glykämischer Index (GI).

Auf dieser Grundlage beschrieb Nicolai Worm 1999 die heute bekannte LOGIMethode. Primäres Ziel dieser Ernährungsform ist die Senkung der postprandialen Blutzucker- und Insulinkonzentrationen und damit die Vorbeugung einer peripheren Insulinresistenz. Dies soll nicht nur durch eine Minderung des GI, sondern v. a. durch eine Senkung der Kohlenhydratmenge, also der glykämischen Last (GL) erreicht werden. Aus diesem Grund definierte Worm den Begriff LOGI neu als Low Glycemic and Insulinemic Diet (Heilmeyer 2008).

Weitere Ziele der LOGI-Methode sind die Minderung oder Beseitigung von Fettstoffwechselstörungen, die Senkung eines erhöhten Blutdrucks, die Reduktion bzw. das Konstant halten des Körpergewichts und eine hohe Nährstoffversorgung - und das mit Genuss und hoher Lebensqualität (Heilmeyer 2008).

Die praktische Umsetzung wird anhand der vierstufigen LOGI-Pyramide erläutert.

Die Basis bilden Lebensmittel mit geringer blutzuckersteigernder Wirkung. Hierzu zählen beispielsweise stärkearmes Gemüse, Salate und Obst (v. a. Beeren). Hiervon kann täglich und reichlich verzehrt werden (Küpper 2011).

Außerdem wird auf eiweißreiche Nahrungsmittel und Fette hoher Qualität (enthalten viele einfach ungesättigte Fettsäuren und Omega-3-Fettsäuren) großen Wert gelegt (Heilmeyer 2008).

Die zweite Stufe bilden tierische sowie pflanzliche Proteinlieferanten. Auch sie rufen nur einen geringen Blutzuckeranstieg hervor und können demnach ebenfalls täglich verzehrt werden. Wichtige Beispiele hierfür sind qualitativ hochwertiges Fleisch, Geflügel und Fisch. Bei Milchprodukten sind aufgrund des geringen GI Sauermilchprodukte zu bevorzugen. Außerdem kommen Eier, Nüsse und Hülsenfrüchte hinzu.

\footnotetext{
2 Die Marke LOGI sowie die LOGI-Methode sind für die systemed GmbH, 44534 Lünen, geschützt.
} 


\section{Glykämischer Index (GI)}

„Der Glykämische Index (GI) unterteilt kohlenhydrathaltige Lebensmittel nach ihrer blutzuckersteigernden Wirkung. [...] [Er] wurde bereits in den 80er Jahren im Rahmen der Diabetes-Forschung entdeckt", (Diabetes Austria, S. 1) und fußt auf der Erkenntnis, dass gleiche Kohlenhydratmengen unterschiedlicher Lebensmittel zu unterschiedlichen Blutzucker- bzw. Insulinanstiegen im Blut führen.

Lebensmittel, die einen entsprechend hohen Anstieg nach sich ziehen, haben einen hohen GI, Lebensmittel, deren blutzuckersteigernde Wirkung eher gering ist, haben demnach einen geringen GI. Die Angabe des Gl erfolgt in Prozent bezogen auf die Fläche unter der Blutzuckerkurve von $50 \mathrm{~g}$ Glucose (Küpper 2011, Strohm 2013).

„Als hoch gelten $\mathrm{Gl}-$ Werte von über 70 , als mittel von 55 bis 70 und als niedrig von unter 55“ (Küpper 2011, S. 488). Da der GI für die Blutzuckerreaktion auf 50 g Glucose steht, damit übliche Verzehrgewohnheiten nicht berücksichtigt werden und er zudem keine konstante Größe darstellt, sondern verschiedenen Einflüssen wie z. B. der Zusammensetzung der Mahlzeiten unterliegt, ist der GI für die alltägliche Ernährung wenig praxisgerecht (Diabetes Austria, Küpper 2011).

Glykämische Last (GL)

Da das Ausmaß der glykämischen Antwort auch von der Kohlenhydratmenge abhängt, wurde zusätzlich zum GI der Begriff der glykämischen Last definiert.

Dieser stellt „das Produkt des GI und der verwertbaren Kohlenhydratmenge (in Gramm) pro Portion eines Lebensmittels, dividiert durch 100 dar" (Strohm 2013, S. 27).

$$
G L=(G I \times \text { verzehrte verwertbare Kohlenhydrate }[\mathrm{g}]) / 100
$$




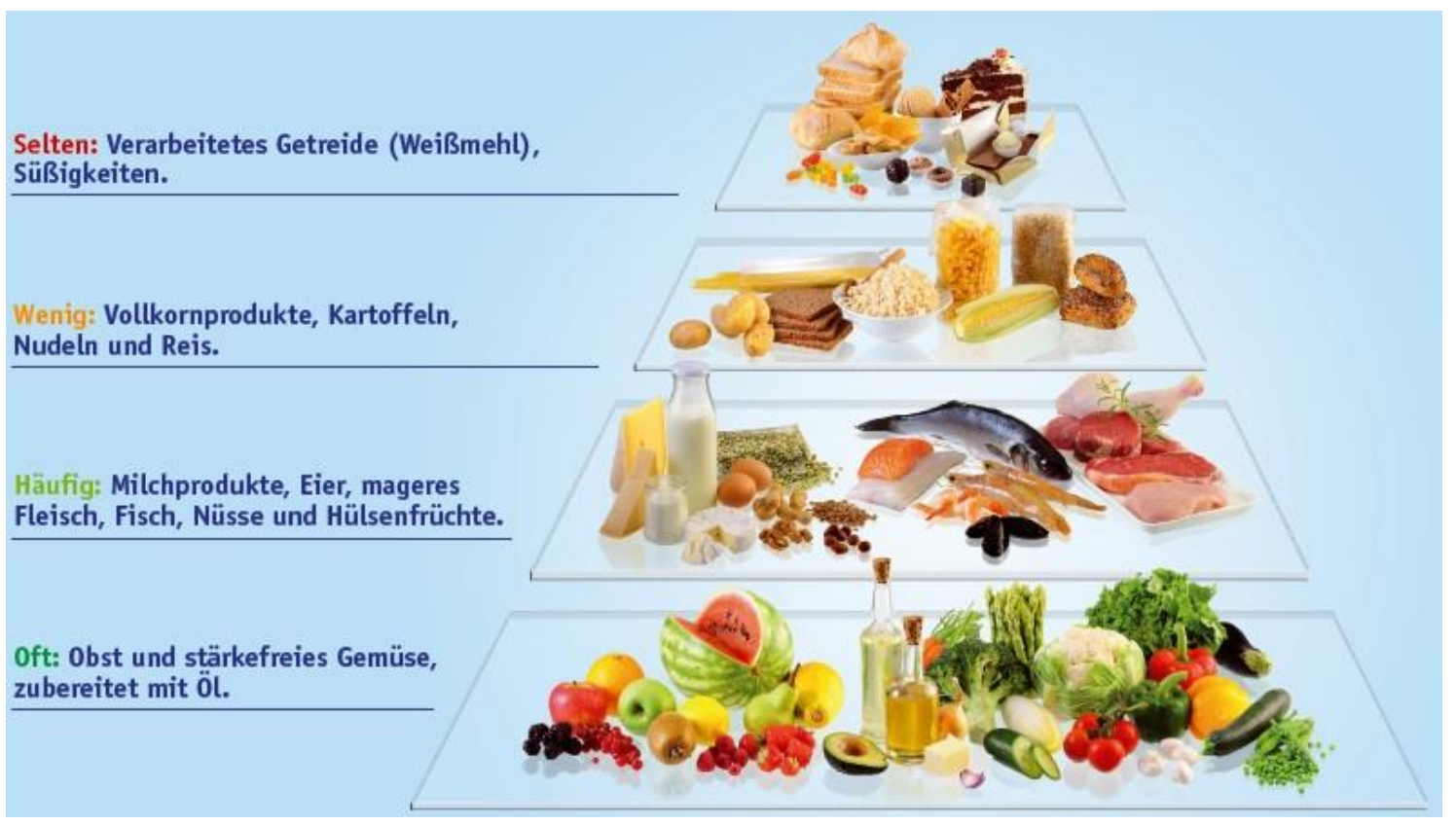

Abbildung 11: Die Original-LOGI-Pyramide nach Dr. Nicolai Worm, Stand 2016, publiziert in den Büchern zur LOGI-Methode bei systemed / www.systemed.de (Die Verwendung der Abbildung erfolgt mit freundlicher Genehmigung des systemed Verlags)

Auf der dritten Stufe befinden sich Lebensmittel mit bereits deutlich stärkerer Blutzuckerwirkung. Je übergewichtiger und weniger sportlich man ist, desto weniger sollte hiervon verzehrt werden. Klassische Vertreter sind Vollkornprodukte wie Reis und Nudeln, aber auch Kartoffeln zählen hierzu.

Die Lebensmittel an der Pyramidenspitze bewirken einen sehr starken Blutzuckeranstieg und sollten demnach nur äußerst selten verzehrt werden. Beispiele hierfür sind Gebäck, jegliche Süßwaren und zuckergesüßte Getränke (Küpper 2011).

Das Besondere der LOGI-Methode ist, dass keine bewusste Kalorienrestriktion eingehalten werden muss, sondern dass ad libitum, d. h. nach Belieben bei Hungergefühl gegessen werden kann. Durch den relativen Anstieg von Protein- und Fettanteil an der zugeführten Nahrung, ist durch deren hohe Sättigungswirkung und der daraus resultierenden Verminderung der Anzahl und Menge der Mahlzeiten letztlich eine dauerhaft niedrigere Kalorienzufuhr gewährleistet.

Dadurch kann nicht nur einer Gewichtszunahme vorgebeugt, sondern sogar effektiv ein Gewichtsverlust erzielt werden (Heilmeyer 2008, Küpper 2011). 
Zusammenfassend lässt sich sagen, dass die LOGI-Methode eine kohlenhydratreduzierte Ernährungsform mit folgender Energieverteilung darstellt:

20 - 25\% Kohlenhydrate, 45 - 50\% Fett und 25 - 30\% Proteine

\subsubsection{Die Standard-Diät nach den Empfehlungen der Deutschen Gesellschaft für Ernährung e.V. (DGE)}

„Vollwertig essen [und trinken] hält gesund, fördert Leistung und Wohlbefinden und unterstützt einen nachhaltigen Ernährungsstil“ (Deutsche Gesellschaft für Ernährung e.V. 2013, S. 1).

Für die Umsetzung dieses Mottos gibt die DGE Empfehlungen aus, die z. T. erheblich von den beiden vorab beschriebenen Ernährungsformen abweichen bzw. sich sogar konträr zu diesen darstellen.

Für eine vollwertige Mischkost nach DGE sollte demnach der Kohlenhydratanteil an der Gesamtenergiezufuhr mehr als 50\% betragen, während sich der Fettanteil (bei Erwachsenen ab 19 Jahren) auf maximal 30\% beschränken sollte (Deutsche Gesellschaft für Ernährung e.V. 2016a).

Eine genaue Auflistung aller Referenzwerte je nach Alter und Geschlecht ist auf der Homepage der DGE (www.dge.de) unter der Rubrik Referenzwerte zu finden.

Als einfache und schnelle Orientierungshilfe für eine gesundheitsbewusste Lebensmittelauswahl hat die DGE den in Abbildung 12 dargestellten Ernährungskreis entwickelt (Deutsche Gesellschaft für Ernährung e.V. 2016b).

„Der Ernährungskreis der Deutschen Gesellschaft für Ernährung e. V. (DGE) dient als Wegweiser für eine vollwertige Ernährung. Er teilt das reichhaltige Lebensmittelangebot in sieben Gruppen ein und erleichtert so die tägliche Lebensmittelauswahl. Die Größe der Kreissegmente verdeutlicht das Mengenverhältnis der einzelnen Lebensmittelgruppen zueinander: Je größer ein Kreissegment ist, desto größere Mengen sollten täglich aus der Gruppe verzehrt werden. Lebensmittel aus kleinen Segmenten sollten sparsam verwendet werden. Für eine gesundheitsfördernde, vollwertige Ernährung 
wird empfohlen, täglich Lebensmittel aus allen sieben Gruppen zu verzehren, das dargestellte Mengenverhältnis zu berücksichtigen und innerhalb der Gruppen zwischen

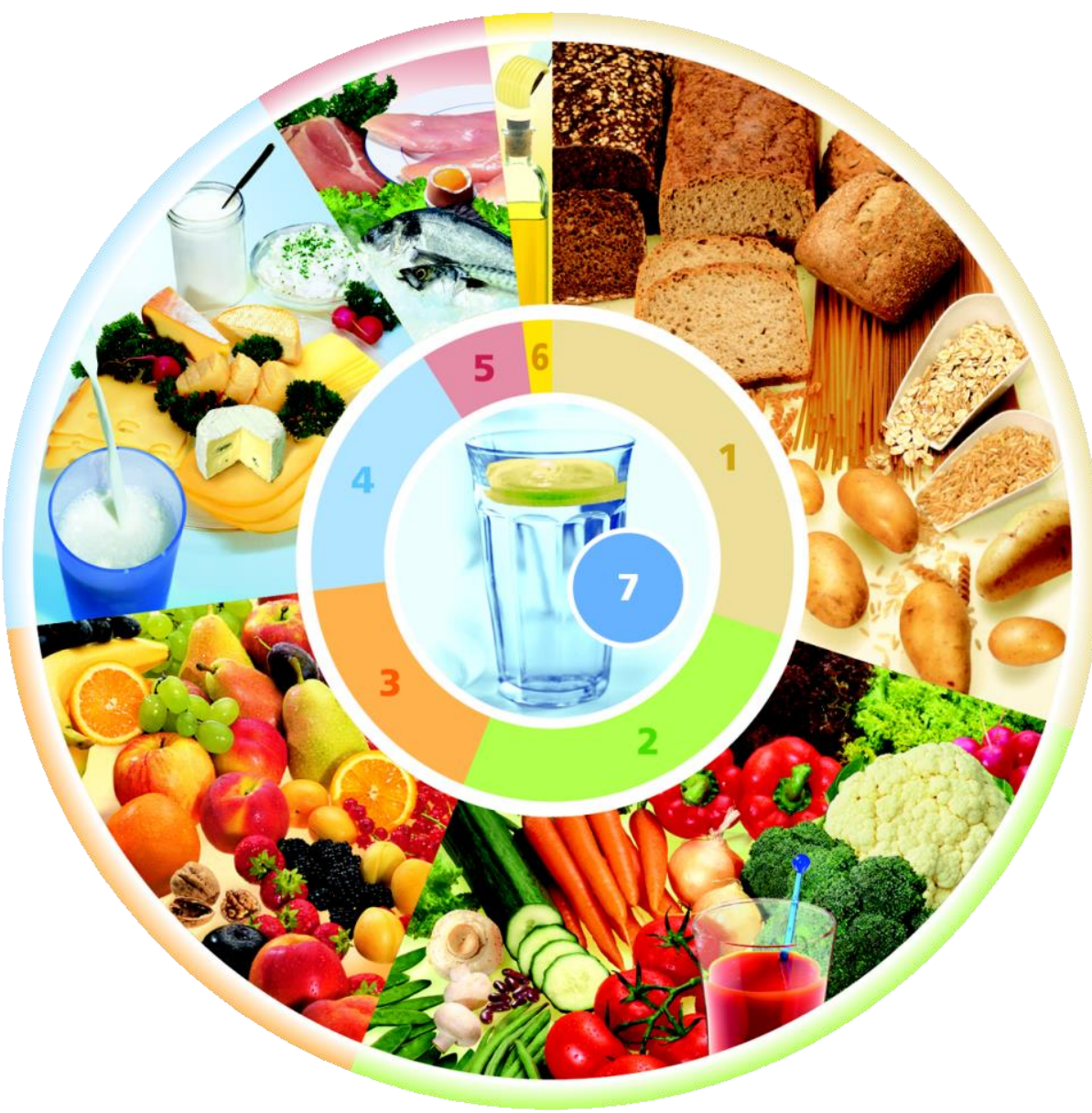

Abbildung 12: DGE-Ernährungskreis ${ }^{\circledR}$, Copyright: Deutsche Gesellschaft für Ernährung e. V., Bonn.

(Diese Abbildung muss in Kombination mit dem hier zitierten und von der DGE formulierten Begleittext erscheinen. Der Begleittext durfte inhaltlich nicht verändert werden.)

den Lebensmitteln abzuwechseln. Ist die Zusammenstellung an einem Tag nicht ausgewogen, dann sollte an den folgenden Tagen bewusst vollwertig ausgewählt und gegessen werden. Auf die Wochenbilanz kommt es an.

Getränke bilden mit einer täglichen Trinkmenge von 1,5 I mengenmäßig die größte Lebensmittelgruppe. Danach folgen die pflanzlichen Lebensmittel Getreideprodukte, Kartoffeln, Gemüse sowie Obst. Sie stellen die Basis einer vollwertigen Ernährung dar 
und liefern Kohlenhydrate, reichlich Vitamine, Mineralstoffe, Ballaststoffe und sekundäre Pflanzenstoffe. Tierische Lebensmittel - möglichst fettarm - ergänzen in kleineren Portionen den täglichen Speisenplan. Sie versorgen den Körper mit hochwertigem Protein, Vitaminen und Mineralstoffen. Fett und fettreiche Lebensmittel sollten eher selten verzehrt werden. Dabei ist die Qualität entscheidend, denn hauptsächlich liefern pflanzliche Öle die essenziellen Fettsäuren.

Deckt die tägliche Ernährungsweise alle Gruppen des Ernährungskreises in den empfohlenen Mengen ab, bleibt Raum für kleine „Extras“, wie Süßigkeiten oder gesalzene Knabberartikel. Auch wenn diese Lebensmittel wenig essenzielle Nährstoffe, dafür aber meistens viel Fett und Zucker enthalten. Solange die Energie- und Nährstoffbilanzen stimmen, ist nichts gegen den bewussten Genuss dieser Lebensmittel einzuwenden“ (Deutsche Gesellschaft für Ernährung e.V. 2014).

Um genussvoll und gesund erhaltend zu essen, hat die DGE zusätzlich auf der Basis wissenschaftlicher Erkenntnisse 10 Regeln formuliert (Deutsche Gesellschaft für Ernährung e.V. 2013). Diese decken sich zum Großteil mit den Inhalten des eben aufgeführten Begleittextes und sind in übersichtlicher Form ebenfalls auf der Homepage der DGE dargestellt.

Erwähnenswert hieraus, da im Begleittext nicht aufgeführt, ist die Empfehlung, täglich mindestens fünf Portionen Gemüse und Obst am Tag zu verzehren. Möglichst frische, nur kurz gegarte und saisonale Produkte sind zu jeder Hauptmahlzeit gewünscht und als Zwischenmahlzeit erlaubt. Dadurch soll das Risiko für ernährungsmitbedingte Krankheiten verringert werden (Deutsche Gesellschaft für Ernährung e.V. 2013).

Insgesamt empfiehlt die DGE also wenig Fett und fettreiche Lebensmittel (ca. 60 - $80 \mathrm{~g}$ Fett pro Tag). Diese liefern zwar essentielle Fettsäuren und fettlösliche Vitamine, können aber auch durch ihre hohe Energiedichte die Entstehung von Übergewicht fördern. Für die Regulierung des Körpergewichts ist deshalb neben vollwertiger Ernährung zusätzlich viel körperliche Bewegung und Sport von enormer Wichtigkeit (Deutsche Gesellschaft für Ernährung e.V. 2013). 


\section{Material und Methoden}

\subsection{Grundlagen}

\subsubsection{Körperkompartiment-Modelle}

Grundvoraussetzung für das Verständnis der in der KOLIBRI-Studie eingesetzten Messmethoden zur Erfassung der Körperzusammensetzung ist das Wissen über die Einteilung des menschlichen Organismus in verschiedene Kompartiment-Modelle (Reimers et al. 2005). Da es im lebenden Menschen keine direkte Methode zur Bestimmung der Körperzusammensetzung gibt, muss auf indirekte Verfahren zur Messung von Teilkomponenten ausgewichen werden, anhand derer dann die Körperzusammensetzung berechnet werden kann (Spaniol 2004).

In Abbildung 13 sind die verschiedenen Körperkompartimente und die ihnen zugrundeliegenden Messmethoden schematisch dargestellt.

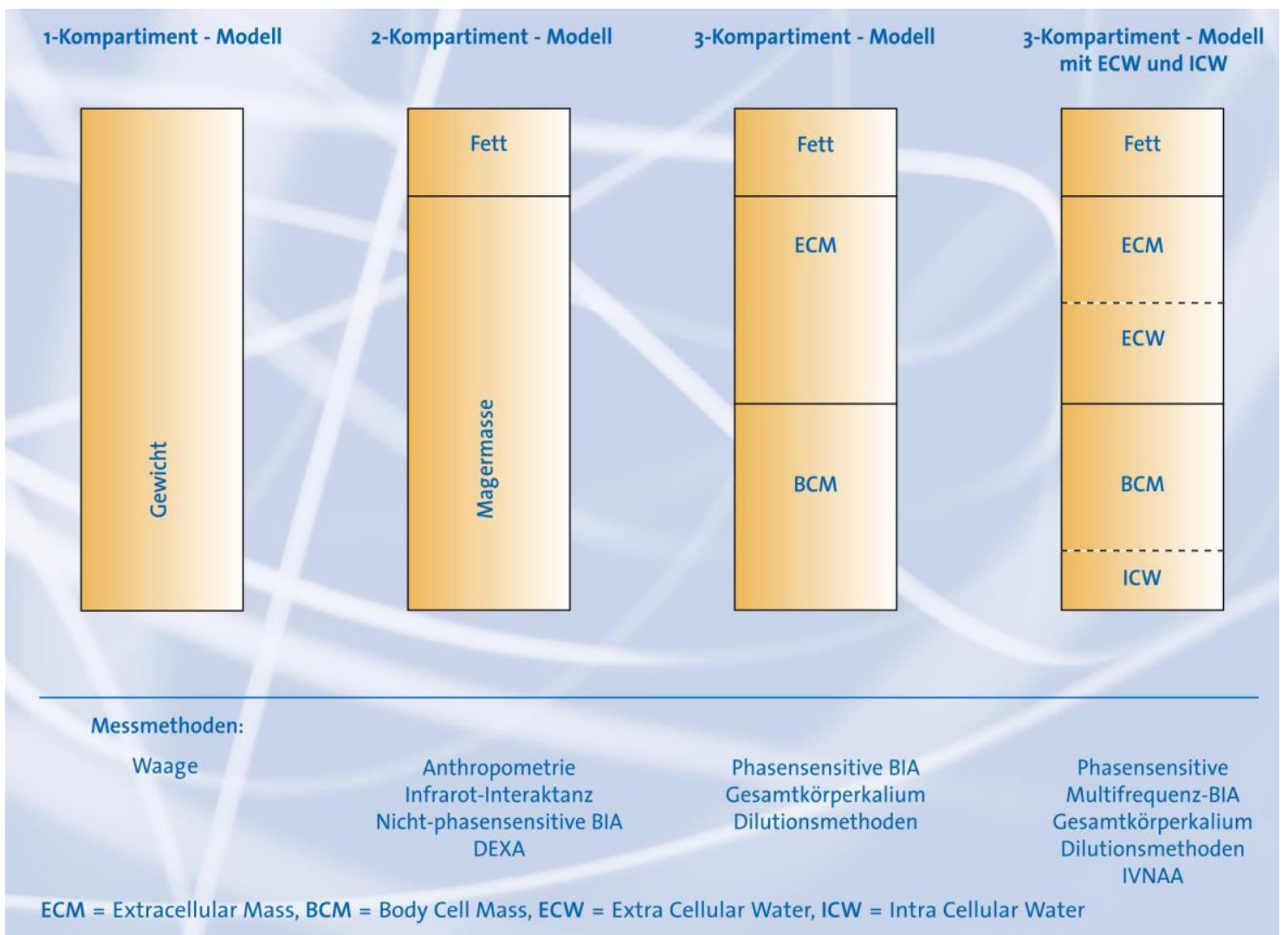

Abbildung 13: Schematische Darstellung der Kompartimentmodelle (Die Verwendung der Abbildung erfolgt mit freundlicher Genehmigung der Data Input $\mathrm{GmbH}$ ) 
Die Bestandteile und Bezeichnungen dieser Abbildung sollen in den folgenden Abschnitten erläutert werden.

\section{Ein-Kompartiment-Modell}

Das Ein-Kompartiment-Modell stellt als gröbster Parameter die Gewichtsmessung des Körpers dar, woraus man einen genau bestimmbaren und sehr wichtigen Messwert erhält.

Leider kann dadurch nur eine grobe Differenzierung bei Gewichtsveränderungen angezeigt und keine Aussage über eine mögliche Ursache getroffen werden (Data Input 2005, Spaniol 2004).

\section{Zwei-Kompartiment-Modell}

Dieses Modell unterteilt den Körper in die beiden Kompartimente Fettmasse (FM = fat mass) und fettfreie Masse (FFM = fat free mass). Im klinischen Sprachgebrauch wird die FFM häufig mit der Magermasse (LBM = lean body mass) gleichgestellt, obwohl diese nicht identisch sind. „Die FFM stellt den Rückstand nach Ether-Extraktion von Fett dar", während die LBM die Differenz aus Körpergewicht und Fettgewebe bildet. Hierbei wird allerdings nicht berücksichtigt, dass das Fettgewebe nicht nur aus Fett, sondern auch zu kleinen Teilen aus Wasser und Proteinen besteht. D. h. im Umkehrschluss, dass das Körpergewicht abzüglich der FFM genaugenommen nicht das Fettgewebe, sondern die Neutralfette ergibt.

Da der Unterschied bezogen auf die Zellmasse jedoch sehr gering ist, werden die beiden Begriffe LBM und FFM auch in dieser Arbeit als Synonyme betrachtet (Reimers et al. 2005, S. 356; Spaniol 2004).

\section{Drei-Kompartiment-Modell}

Das 3-Kompartiment-Modell entsteht durch die weitere Unterteilung der FFM in die Körperzellmasse $(\mathrm{BCM}=$ body cell mass $)$ und die extrazelluläre Masse $(\mathrm{ECM}=$ extracellular mass) (Data Input 2005).

Der Ermittlung der BCM kommt durch ihre hohe metabolische Aktivität ernährungsphysiologisch eine bedeutende Rolle zu, da sie aus metabolischer Sicht sowohl für die Stoffwechselaktivität, als auch für den Kalorienverbrauch des Organismus von entscheidender Bedeutung ist. „Zur BCM gehören [u. a] die Zellen der Skelettmuskulatur und der inneren Organe inkl. des intrazellulären Wassers" (Spaniol 2004, S. 4). 
Die ECM besteht zum einen aus flüssigen Bestandteilen wie beispielsweise Plasmavolumen, interstitielle und transzelluläre Flüssigkeit und zum anderen aus festen Bestandteilen wie beispielsweise Knochenmasse, Faszien und Knorpel (Spaniol 2004).

\section{Drei-Kompartiment-Modell mit ECW und ICW bzw. Vier-Kompartiment-Modell}

Weitere Differenzierungsmöglichkeiten sind wie in Abbildung 13 gezeigt das DreiKompartiment-Modell mit extrazellulärem Wasser (ECW = extracellular water) und intrazellulärem Wasser (ICW = intracellular water) oder auch das in Abbildung 14 gezeigte Vier-Kompartiment-Modell.

Wichtigster Parameter in diesen beiden Modellen und v. a. für die bioelektrische Impedanzanalyse und deren Berechnungen von großer Bedeutung, ist das Gesamtkörperwasser (TBW = total body water), dessen Menge im Organismus in erster Linie über die BCM und damit primär über die Muskelmasse determiniert wird. Es setzt sich wiederum aus ECW und ICW zusammen (Data Input 2005).

\begin{tabular}{|c|c|c|c|c|c|}
\hline $\begin{array}{l}\text { Min- } \\
\text { eral- } \\
\text { ien }\end{array}$ & Protein & \multicolumn{3}{|l|}{ Wasser } & Triglyzeride \\
\hline \multicolumn{2}{|c|}{ Skelett } & \multicolumn{2}{|c|}{ Skelettmuskel } & $\begin{array}{l}\text { übriges } \\
\text { Gewebe }\end{array}$ & Fett \\
\hline \multicolumn{3}{|c|}{ ECM } & \multicolumn{2}{|l|}{$\mathrm{BCM}$} & Fett \\
\hline
\end{tabular}

Abbildung 14: Ausschnitt aus „Modellvorstellung zur Körperzusammensetzung des Menschen" (Abbildung modifiziert nach Spaniol 2004, S. 3)

\subsubsection{Dualenergie-Röntgen-Absorptiometrie}

Die Dualenergie-Röntgen-Absorptiometrie (DXA/DEXA; engl: Dual-energy X-ray absorptiometry) ist ein ursprünglich für die Messung der Knochendichte im Rahmen der Osteoporosediagnostik beim Menschen entwickeltes Verfahren, das heutzutage jedoch auch gerne zur Bestimmung der Körperzusammensetzung eingesetzt wird (Scholz und Baulain 2009). 
Die Ursprünge der DXA liegen in den 1960er Jahren, wo erstmals mittels single-photonabsorptiometry der Knochenmineralgehalt gemessen werden konnte. Durch die Weiterentwicklung zur dual-photon-absorptiometry konnte dann gleichzeitig der Magergewebegehalt bestimmt werden.

Bei der heutigen DXA, die 1995 die FDA-Freigabe für den klinischen Einsatz zur Messung der Körperzusammensetzung erhielt (Pandey et. al 2011), wurde die natürliche Strahlenquelle durch einen konventionellen Röntgengenerator ersetzt. Mittels nachgeschaltetem Filter können damit aus dem gesamten Röntgenspektrum die gewünschten Maxima herausgefiltert werden, wodurch eine höhere Präzision, eine deutlich kürzere Scanzeit und v. a. eine geringere Strahlenbelastung für die Patienten erreicht werden kann (Schöllhorn 2007).

Außerdem zur erheblichen Verkürzung der Scanzeit beigetragen hat die Weiterentwicklung der DXA-Technik in Sachen Geometrie der erzeugten Strahlung. Dahinter steckt die Art und Weise, wie der Körper von den Röntgenstrahlen durchdrungen wird. Geräte der 1. Generation verwendeten noch einen punktförmigen Röntgenstrahl, der den Körper geradlinig durchstrahlte (pencil beam; siehe Abbildung 15) und nur von einem einzigen Detektor registriert wurde. Somit war die Scan-Zeit mit 10 - 20 Minuten dementsprechend lang.

Bei den heute verwendeten Geräten wird ein bestimmter fächerförmiger Röntgenstrahl (narrow fan-beam; siehe Abbildungen 16 \& 17) emittiert, der in einem Feld mit vielen hochauflösenden Detektoren registriert wird. Dadurch konnte die Scan-Zeit auf mittlerweile 5 - 7 Minuten für ein Ganzkörper-Scan reduziert werden (Schöllhorn 2007, Toombs et al. 2012).

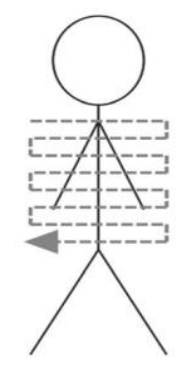

Abbildung 15: Pencil-beam

(Die Verwendung der Abbil-

dung erfolgt mit freundlicher

Genehmigung von John Wiley

and Sons und Copyright

Clearance Center)

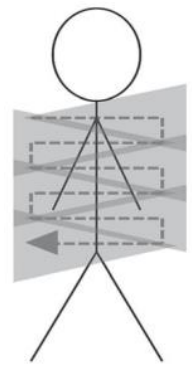

Abbildung 16: Narrow fanbeam (Die Verwendung der Abbildung erfolgt mit freundlicher Genehmigung von John Wiley and Sons und Copyright Clearance Center) 


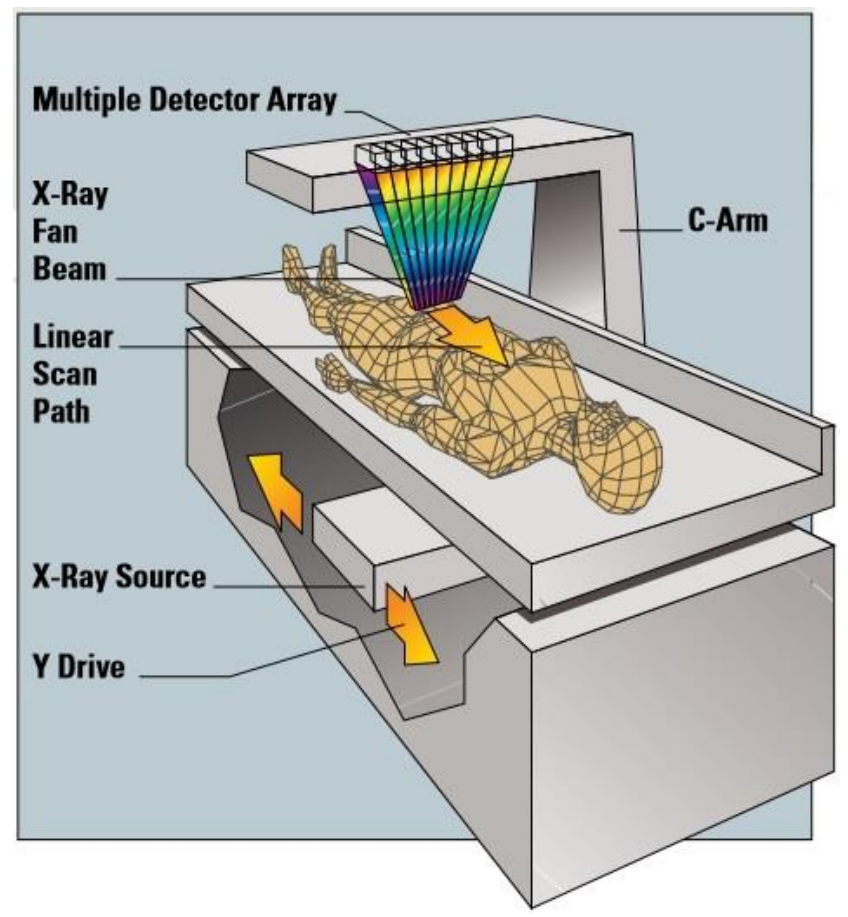

Abbildung 17: Schemazeichnung DXA-Gerät (Die Verwendung der Abbildung erfolgt mit freundlicher Genehmigung der MMS MEDICOR MEDICAL SUPP-

LIES GmbH)

Grundsätzlich - unabhängig von welchem Hersteller - besteht das DXA-Gerät aus einem Untersuchungstisch, auf dem der Patient / die Patientin positioniert wird und einem darüber angebrachten mobilen Arm (siehe Abbildung 17).

Die Röntgenquelle kann sich dabei entweder im Inneren des Tisches oder im mobilen Arm befinden, entscheidend ist nur, dass sich Röntgenquelle und Detektor stets auf einer Ebene befinden und der Detektor so ständig die Strahlung bzw. deren Abschwächung messen kann.

Mittels Computeranlage und Software werden die entsprechenden Berechnungen durchgeführt und die daraus entstehenden Bilder angezeigt (Bernau 2011, Schöllhorn 2007). Das Prinzip der DXA beruht darauf, dass die verschiedenen Gewebe des Körpers aufgrund ihrer physikalischen Dichte in Abhängigkeit von der verwendeten Energie der Röntgenstrahlung zu einer unterschiedlichen Schwächung dieser Strahlung führen (Oehme 2013). Im Gegensatz zu herkömmlichen digitalen Röntgenverfahren werden beim DXA-Verfahren zeitgleich zwei energetisch unterschiedliche Röntgenstrahlen ausgesendet (Dekowski 2015), wodurch für jeden Messpunkt zwei charakteristische Schwächungswerte vom Detektor registriert werden (Oehme 2013). Dadurch können 
Materialien und Gewebe im Gegensatz zum konventionellen Röntgen, bei dem nur Strahlen mit der gleichen Energie zur gleichen Zeit abgegeben werden, genauer untersucht und klassifiziert werden (siehe Abbildung 18) (Dekowski 2015).

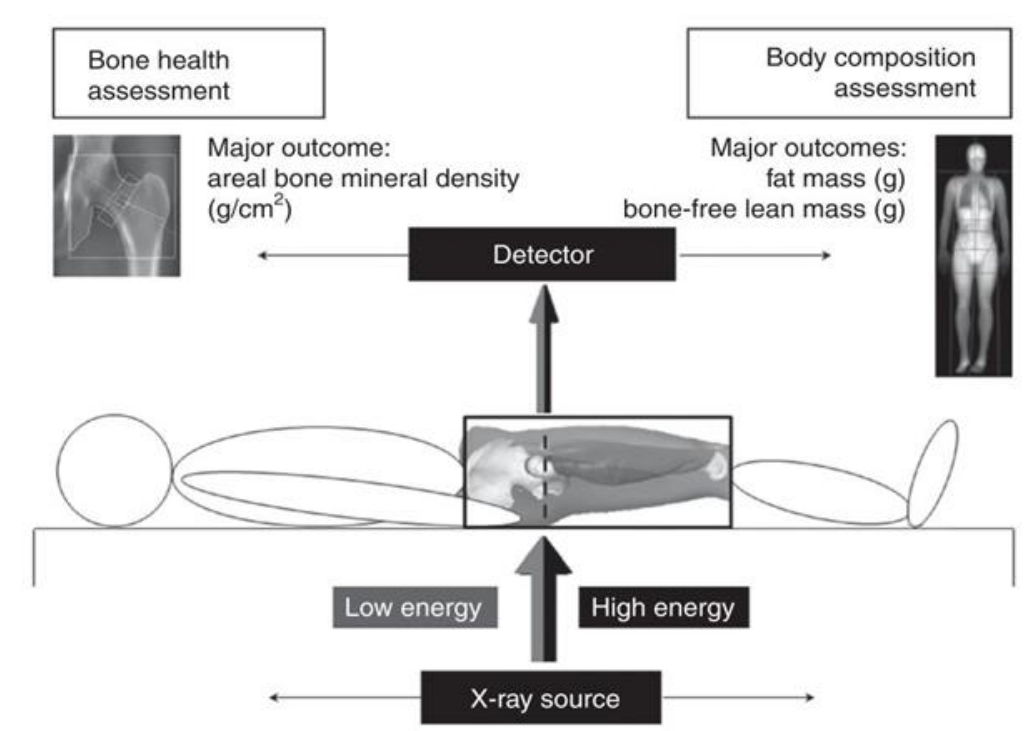

\begin{abstract}
Abbildung 18: Grundsätzliches Prinzip der DXA (Je nach durchstrahltem Gewebe, werden die hoch- und niedrigenergetischen Strahlen unterschiedlich abgeschwächt, woraus dann die verschiedenen Kompartimente berechnet werden können) (Toombs et al. 2012). (Die Verwendung der Abbildung erfolgt mit freundlicher Genehmigung von John Wiley and Sons und Copyright Clearance Center)
\end{abstract}

Durch die niedrigenergetische Strahlung (meist 38 - $40 \mathrm{keV}$ ) wird das den Knochen umgebende Weichgewebe detektiert, während mittels hochenergetischer Röntgenstrahlung (meist $70 \mathrm{keV}$ ) der röntgendichte Knochen erfasst wird (Oehme 2013, Schöllhorn 2007).

Genau genommen wird aus dem Quotienten der Intensitätsabschwächung der beiden Strahlungen bzw. aus der Abschwächung des niedrigen Energieniveaus im Verhältnis zur Abschwächung des höheren Energieniveaus der sogenannte Röntgenschwächungskoeffizient oder auch R-Wert berechnet (Bernau 2011, Schöllhorn 2007). „Dieser RWert dient als Berechnungsgrundlage für die Körperzusammensetzung [...], da für jedes Element, jede Verbindung oder Gewebe ein theoretischer R-Wert existiert" (Schöllhorn 2007, S. 14) (siehe Tabelle 3). Als Orientierung kann man grundsätzlich 
sagen, dass Elemente mit einer hohen Ordnungszahl größere R-Werte besitzen als Elemente mit einer niedrigen Ordnungszahl (Bernau 2011).

Tabelle 3: Theoretische R-Werte ausgewählter chemischer Elemente und Verbindungen (modifiziert nach Pietrobelli et al. 1996, S. 944-945)

\begin{tabular}{|c|c|}
\hline $\begin{array}{c}\text { Chemisches Element bzw. chemische } \\
\text { Verbindung }\end{array}$ & Theoretischer R-Wert \\
\hline Wasserstoff (H) & 1,0891 \\
\hline Kohlenstoff (C) & 1,2199 \\
\hline Stickstoff (N) & 1,3043 \\
\hline Sauerstoff (O) & 1,4167 \\
\hline Fettsäuren & $1,2058-1,2289$ \\
\hline Triglyzeride & $1,2139-1,2333$ \\
\hline Proteine & 1,2906 \\
\hline Wasser & 1,3572 \\
\hline Knochenmineralien & 2,8617 \\
\hline
\end{tabular}

Schließlich lässt sich so aus der Ermittlung der Eingangs- und Ausgangsintensitäten der beiden Röntgenstrahlen und bei bekanntem Schwächungskoeffizienten die Masse bzw. Dichte pro Messpunkt berechnen und mittels Computer darstellen (Krefting 2005).

Für jeden Probanden werden so folgende Daten erhoben (Bernau 2011):

- Knochenmineraldichte $\left[\mathrm{g} / \mathrm{cm}^{2}\right]$ (engl.: $\mathrm{BMD}=$ bone mineral density)

- Knochenmineralgehalt [g] (engl.: $\mathrm{BMC}=$ bone mineral content)

- Fettgewebe [g und \%]

- Magergewebe [g und \%] (= Magermasse LBM)

Die größte Bedeutung besitzt die DXA weiterhin in der Osteoporosediagnostik. Es ist jedoch auch interessant zu sehen, wie die Bestimmung der Körperzusammensetzung mittels DXA immer interessanter für die verschiedensten Bereiche und deshalb auch immer häufiger eingesetzt wird. So wird sie u. a. im Zusammenhang mit Stoffwechselerkrankungen und Ernährungsstörungen, aber auch zur gezielten Diagnostik einer Vermehrung von intraabdominellem Fettgewebe eingesetzt, was bekanntermaßen mit einem erhöhtem Risiko einhergeht, an Herzkranzgefäßerkrankungen oder Typ 2 Diabetes zu erkranken (Schöllhorn 2007). 


\subsubsection{Bioelektrische Impedanzanalyse}

„Die bioelektrische Impedanzanalyse (BIA) wird seit ca. 20 Jahren als schnelle und nichtinvasive Methode zur Bestimmung der Körperzusammensetzung eingesetzt" (Edlinger 2002, S. 24).

Aufgrund günstiger Eigenschaften für Patienten und Anwender hat diese Technik inzwischen nicht nur in die Forschung und Wissenschaft, sondern auch in Arztpraxen und Krankenhäusern Einzug gehalten. Hier werden die berechneten Kompartimente zur Bestimmung des Ernährungsstatus und zur Verlaufsbeurteilung von Ernährungstherapien herangezogen (Wirth 2006).

„Grundlage der BIA ist die Tatsache, dass die verschiedenen Gewebe- und Zellarten des menschlichen Körpers [...] Strom unterschiedlich gut leiten“" (Reimers et al. 2005, S. 356). Dazu wird über je zwei an Hand und Fuß positionierte Elektroden ein homogenes, elektrisches Wechselstromfeld mit konstanter Stromstärke in der Messperson erzeugt (Spaniol 2004). Der Körper setzt dem erzeugten Wechselstrom einen Widerstand entgegen, der direkt gemessen und als Impedanz Z bezeichnet wird (Borrmann 2013). Die Impedanz selbst setzt sich wiederum aus zwei Anteilen zusammen:

1. Die Resistance $\mathbf{R}$ stellt den rein ohmschen Widerstand des Wasser- und Ionenanteils im menschlichen Körper dar. „Da hauptsächlich die Magermasse aus leitfähigem Gewebe, $d$. h. aus elektrolythaltigem Wasser besteht, während das Fettgewebe als Isolator wirkt, korreliert die Resistance invers mit dem Gesamtkörperwasser [...]" (Voigt 2009, S. 8-9).

2. Die Reactance Xc beschreibt den kapazitiven Widerstand, der durch die Kondensatoreigenschaften der Körperzellmembranen dem Strom entgegengesetzt wird (Data Input 2005). Da man sich den menschlichen Körper als eine große Anzahl von Einzelkondensatoren vorstellen kann, ist die Reactance ein Maß für die Körperzellmasse BCM (Spaniol 2004).

Mit modernen, phasensensitiven BIA-Geräten lassen sich die beiden Komponenten der Impedanz getrennt erfassen (Spaniol 2004). Die Kondensatoreigenschaften der Zellmembranen bedingen eine Zeitverschiebung zwischen Stromstärken- und Spannungsmaximum und führen zu einer direkt messbaren Phasenverschiebung (Stiedenroth 
2009). Diese wird als Phasenwinkel alpha bezeichnet und in Grad angegeben (Spaniol 2004) (siehe Abbildung 19).

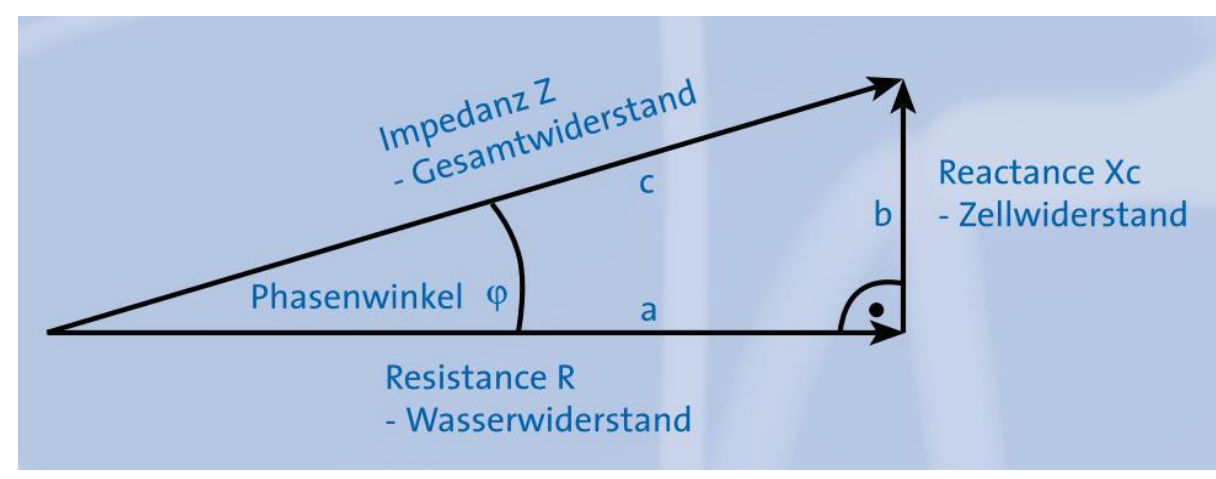

Abbildung 19: Die Bestimmung von R und Xc aus Impedanz und Phasenwinkel (Die Verwendung der Abbildung erfolgt mit freundlicher Genehmigung der Data Input GmbH)

Je mehr Zellmembranen, sprich Zellmasse, der Strom passieren muss, umso größer ist dementsprechend die Phasenverschiebung (Stiedenroth 2009). Daraus folgt, dass reine Zellmembranmasse einen Phasenwinkel von $90^{\circ}$ hätte, während Elektrolytwasser einen Phasenwinkel von $0^{\circ}$ aufweisen würde (Spaniol 2004). Als normwertig gilt der Bereich zwischen 5,0 und 9,0 Grad. Durch die Ableitung des Phasenwinkels können zum einen Aussagen über die Integrität der Zellmembranen (Stiedenroth 2009) und zum anderen Aussagen über den Gesundheitszustand des Organismus getroffen werden (Data Input 2005). Dies soll durch die folgenden beiden Abbildungen 20 und 21 verdeutlicht werden:

Intakte Zelle mit hoher Membranintegrität oder hohe Zelldichte hoher Phasenwinkel

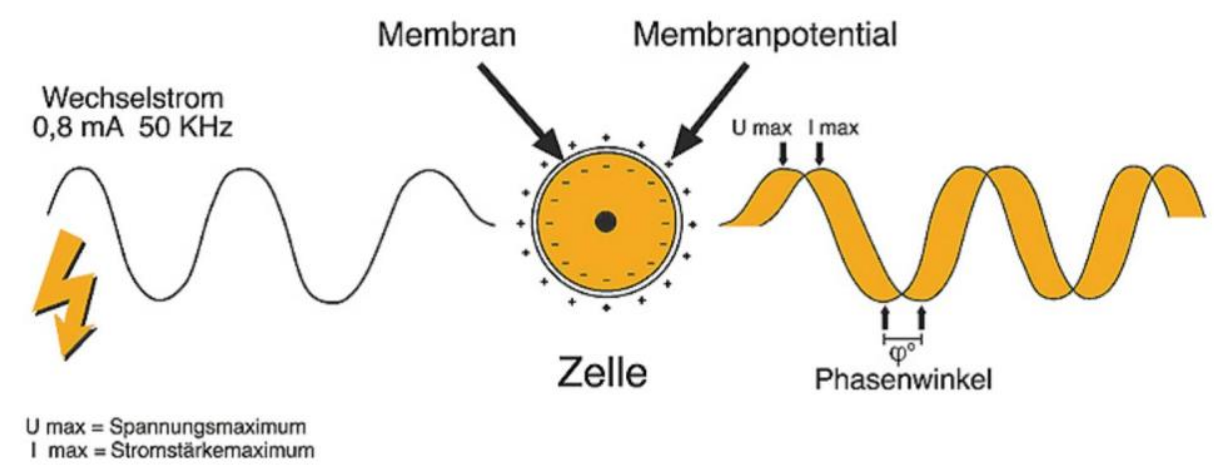


Geschädigte Zelle oder niedrige Zelldichte niedriger Phasenwinkel

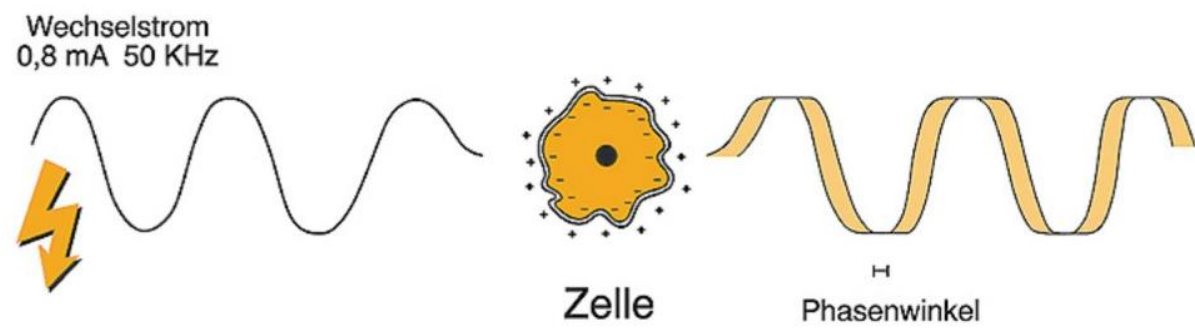

Abbildung 21: Die Entstehung des Phasenwinkels an der Zellmembran - Geschädigte Zelle (Die Verwendung der Abbildung erfolgt mit freundlicher Genehmigung der Data Input $\mathrm{GmbH})$

Die bioelektrische Impedanzanalyse stellt eine indirekte Methode zur Bestimmung der Körperzusammensetzung dar, da die direkt gemessenen Parameter Impedanz und Phasenwinkel als Grundlage zur Berechnung der Körperkompartimente dienen.

Ausgehend von Abbildung 22 sollen die Berechnungen der einzelnen Kompartimente erläutert werden:

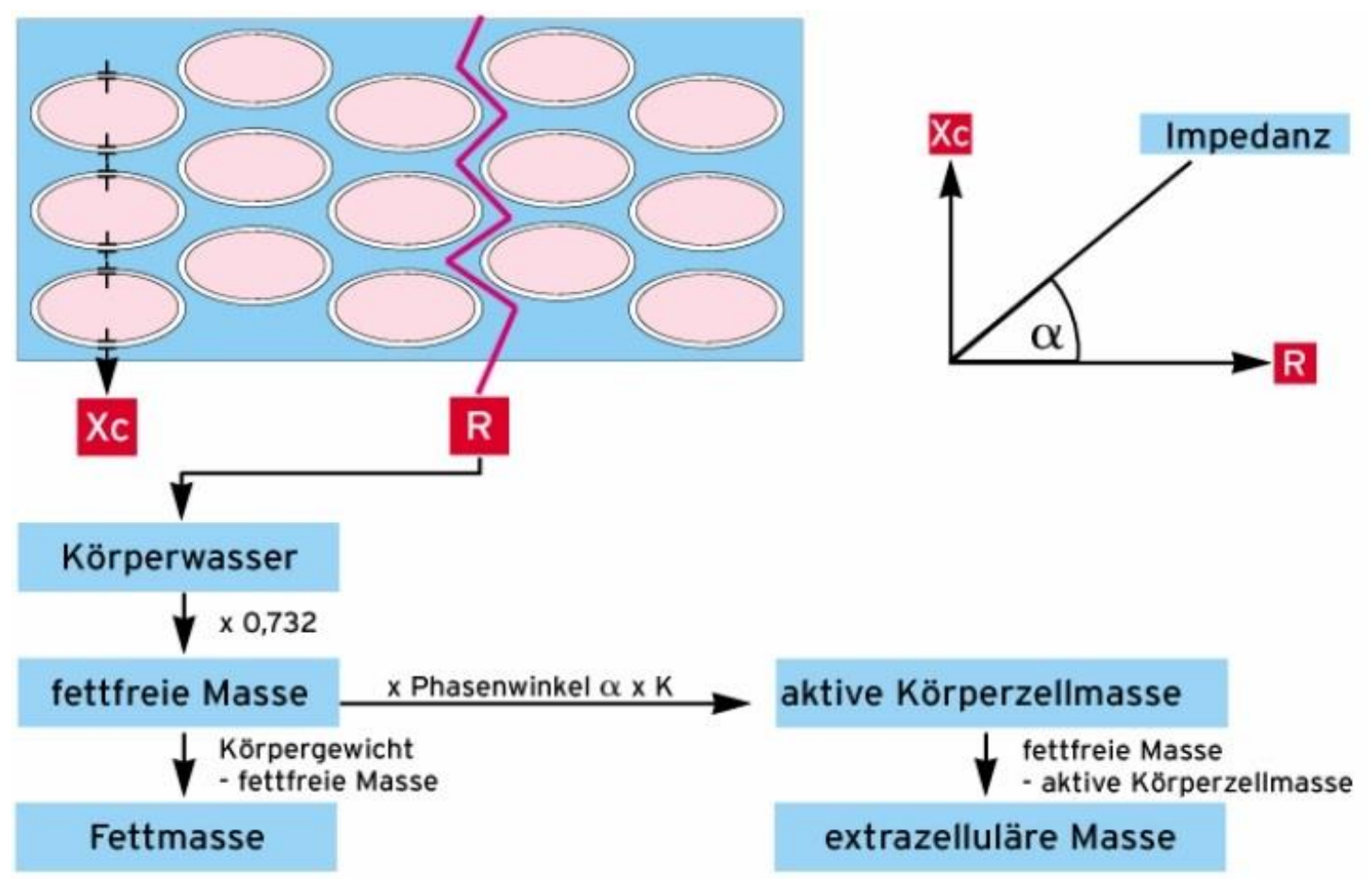

Abbildung 22: BIA-Berechnung (Bardenhagen 2005, S.11; Die Verwendung erfolgt mit freundlicher Genehmigung von Prof. Dr. S. Iwanowski - http://www.fh-wedel.de/ iw) 


\section{Ganzkörperwasser (TBW):}

Die BIA stellt das einfachste indirekte Verfahren zur Erhebung des TBW dar, wobei allerdings nur das Wasser im Gewebe erfasst wird. Das bedeutet, dass oral aufgenommenes, vom Körper noch nicht resorbiertes Wasser, nicht gemessen wird, während intravenös verabreichte Lösungen sofort vom Gerät registriert werden (Data Input 2005, Stiedenroth 2009). Wie bereits erwähnt, ist die Resistance umgekehrt proportional zum Gesamtkörperwasser. Dies rührt daher, dass elektrischer Strom nur durch Körperkompartimente fließt, die ionisiertes Wasser enthalten.

Das TBW kann somit folgendermaßen berechnet werden (Stiedenroth 2009):

$$
\begin{array}{l|l}
\text { TBW }=\mathrm{Ht}^{2} / \mathrm{R} & \begin{array}{l}
\mathrm{Ht}=\text { Körpergröße } \\
\mathrm{R}=\text { Resistance }
\end{array}
\end{array}
$$

„Die WHO (World Health Organisation) gibt für normalgewichtige Personen folgende Prozentsätze für den Körperwassergehalt an: Kinder 60 -75\%, Frauen 50 -55\% und Männer 60 -65\%" (Geratherm Medical AG, S. 2). Da die Menge des Körperwassers eines Organismus in erster Linie über die Körperzellmasse BCM und damit primär über die Muskelmenge bestimmt wird (Data Input 2005), können sehr muskulöse Menschen einen prozentualen Anteil des TBW an der Gesamtkörpermasse von bis zu 80\% aufweisen, während dieser sich bei Adipösen auf Werte unter 50\% belaufen kann (Stiedenroth 2009).

Zur weiteren, genaueren Differenzierung des TBW in ECW und ICW ist eine Multifrequenz-Messung notwendig. Das Prinzip soll anhand der Abbildung 23 veranschaulicht werden.

Da niedrige Frequenzen im Bereich von 1 bis $5 \mathrm{kHz}$ die Zellmembranen kaum überwinden können, breiten sie sich nur im Extrazellulärraum aus und enthalten praktisch keinen Reactance-Anteil. Daher kann mit diesen Frequenzen selektiv das ECW berechnet werden (Data Input 2005). Da sich Wellen höherer Frequenzen und damit kürzerer Wellenlängen auch zunehmend im intrazellulären Bereich ausbreiten (Brandt 2008), ist bei Messungen mit $50 \mathrm{kHz}$ oder höher die Resistance proportional zum extra- und intrazellulären Wasser. 
Das ICW lässt sich dadurch ganz einfach aus der Differenz zwischen TBW und ECW berechnen (Spaniol 2004):

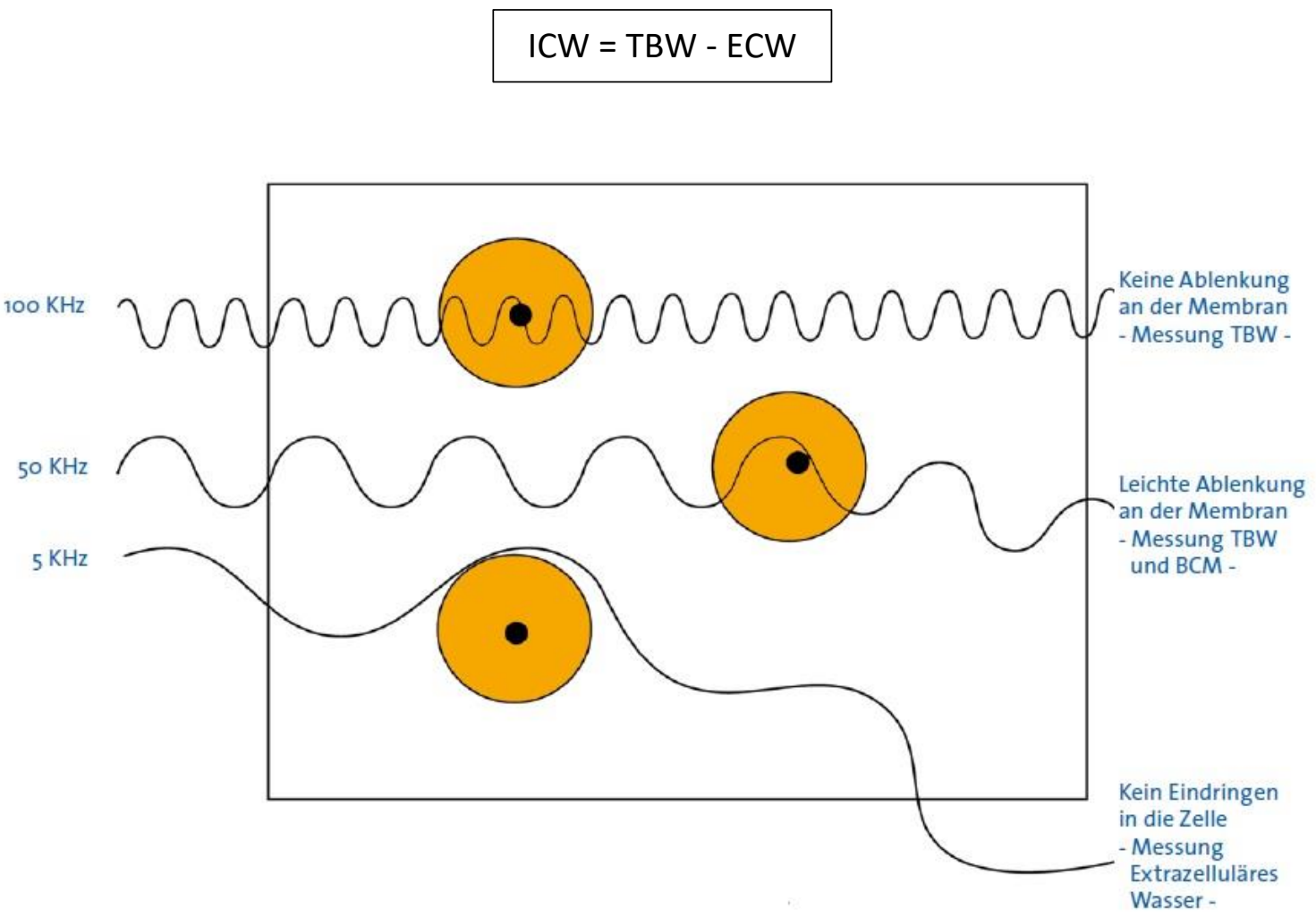

Abbildung 23: Multifrequenzmessung: Die Leitungswege verschiedener Frequenzen im Gewebe (Die Verwendung der Abbildung erfolgt mit freundlicher Genehmigung der Data Input $\mathrm{GmbH})$

\section{Magermasse (LBM) bzw. fettfreie Masse (FFM):}

Die FFM war definiert als Körpergewicht abzüglich des Fettgewebes bzw. der Fettmasse. „Sie leistet den größten Teil der metabolischen Arbeit und ist damit maßgeblich am Energieumsatz des Körpers beteiligt." Ausgehend von der Annahme, dass die FFM beim Gesunden einen konstanten Hydrierungsgrad von 73\% aufweist, kann sie anhand der folgenden Formel berechnet werden (Voigt 2009, S. 10):

$$
\mathrm{LBM}=\mathrm{TBW} \times 0,73
$$

Höhere Werte des Hydrierungsgrades ergeben sich physiologischer Weise bei Kindern, pathologisch erhöhte Werte findet man beispielsweise bei der sog. Anasarka (hier bis zu 85\%). Ein entsprechend niedrigerer Hydrierungsgrad muss bei Exsikkose beachtet werden (Data Input 2005). 


\section{Fettmasse (FM):}

Aufgrund der Dichte von $0,9 \mathrm{~g} / \mathrm{cm}^{3}$ wirkt das Körperfett als Isolator für Wechselstrom. Außerdem besitzt es nicht die typischen Eigenschaften wie die Zellen der BCM und weist daher auch kaum kapazitiven Widerstand auf.

Die Fettmasse kann somit nicht direkt erfasst werden, sie lässt sich allerdings ganz einfach als Gewichtsdifferenz aus Körpergewicht und FFM berechnen (Data Input 2005):

$$
F M=K G-F F M
$$

„Der Ganzkörperfettanteil liegt bei schlanken Männern unter 20\% und bei ebensolchen Frauen unter 25\% der Körpermasse. Bei Adipositas ist dieser Anteil höher und kann 50\% übersteigen“ (Voigt 2009, S. 11).

\section{Körperzellmasse (BCM) und Extrazelluläre Masse (ECM):}

„Die BCM ist die zentrale Größe bei der Beurteilung des Ernährungszustandes eines Patienten, da sämtliche Stoffwechselarbeiten des Organismus innerhalb der Zellen der BCM geleistet werden" (Data Input 2005, S. 12). Für den individuellen Anteil der BCM an der FFM spielen genetische Faktoren wie beispielsweise der Konstitutionstyp, aber auch das Alter und natürlich der Trainingszustand eine entscheidende Rolle. Im Altersbereich von 18 - 75 Jahren sollte der Anteil der BCM an der FFM bei Männern idealerweise zwischen 53 - 59\%, bei Frauen zwischen 50 - 56\% betragen.

Aufgrund der phasensensitiven Elektronik der heutigen BIA-Geräte lassen sich Reactance und Resistance unterscheiden und die BCM kann mit Hilfe des gemessenen Phasenwinkels berechnet werden:

$$
\mathrm{BCM}=\mathrm{FFM} \times \text { Phasenwinkel } \times \mathrm{K} \quad \mathrm{K}=\text { Konstante }
$$

Die Berechnung des letzten Parameters, nämlich der ECM ist nun denkbar einfach:

$$
\mathrm{ECM}=\mathrm{FFM}-\mathrm{BCM}
$$


Wörtlich bedeutet dies, dass die ECM den Anteil der FFM darstellt, der sich außerhalb der Zellen der BCM befindet. Beispiele für feste Anteile der ECM sind bindegewebige Strukturen wie beispielsweise Sehnen, Faszien und Knochen, zu den flüssigen Anteilen zählen u. a. das Plasma und das interstitielle Wasser (Data Input 2005).

\subsubsection{Körperfettwaage}

Körperfettwaagen basieren auf dem oben beschriebenen Prinzip der bioelektrischen Impedanzanalyse. Auch hier liefert schwacher und von außen angelegter Wechselstrom aufgrund der unterschiedlichen Leitfähigkeiten der Körpergewebe Informationen über den Widerstand der einzelnen Kompartimente. Aus den gemessenen Widerständen und den Zahlenwerten für Größe, Gewicht, Geschlecht und Alter können dann die Kompartimente Körperwasser, Körperfett, Muskelmasse und Knochenmasse ermittelt werden.

Da sich die Elektroden zur Messung auf der Oberfläche der Waage befinden, worauf sich der Proband / die Probandin dann mit den nackten Füßen stellt, wird nur der Widerstand von einem Fuß zum anderen und damit insgesamt nur vom Unterkörper gemessen. Anschließend werden die Ergebnisse auf den gesamten Körper hochgerechnet (Zok 2014).

Solch eine Waage mit diesem Messprinzip wurde auch hier im Rahmen der KOLIBRIStudie verwendet.

Modernere Körperfettwaagen, die Kontakte für Hände und Füße besitzen und somit dem Grundaufbau der oben beschriebenen BIA-Messung entsprechen, sind neben der einfachen Bestimmung der gesamten Fett- und Muskelmasse auch in der Lage, Aussagen über deren Verteilung in Armen, Beinen und Rumpf zu treffen. Hierbei findet somit eine Segment-Analyse statt (Zok 2014).

\subsubsection{Nahinfrarot-Spektroskopie}

Ursprünglich wurde die Nahinfrarot-Spektroskopie (NIRS) im Jahr 1970 vom U.S. Department of Agriculture (US Landwirtschaftsministerium) zur Untersuchung von Getreide und Fleisch auf deren organische Bestandteile entwickelt (Raschka et al. 2006, Schlattmann 2005). Bedeutsam für die Anwendung beim Menschen wurde die Metho- 
de 1977 als gezeigt werden konnte, dass nicht-invasiv mittels nahinfrarotem Licht die Sauerstoffbindung an Hämoglobin im Gehirn durch den intakten Schädelknochen hindurch gemessen werden konnte. Dies wurde zunächst nur an anästhesierten Katzen, später aber auch am Menschen durchgeführt (Haux 2007).

In den darauffolgenden Jahren folgten weitere klinische Studien - u. a. bei Früh- und Neugeborenen - und die NIRS fand immer mehr Eingang in die klinische Praxis (Haux 2007, Wally und Velik-Salchner 2015).

Die heutzutage zur Verfügung stehenden Nahinfrarot-Diagnostiksysteme sind in der Lage, eine genaue Analyse der Körperzusammensetzung durchzuführen und ermöglichen dadurch eine separate Betrachtung und Bewertung der einzelnen Körperkompartimente (DCK med GmbH).

„Die spektroskopische Bestimmung der Körperzusammensetzung [...] basiert auf dem Prinzip von Lichtabsorption und -reflexion im Nahinfrarot-Wellenlängenbereich“ (Scholz und Baulain 2009, S. 91). Dazu wird Licht spezifischer Wellenlänge ins Gewebe emittiert (siehe Abbildung 24), je nach dessen chemischer Zusammensetzung unterschiedlich stark absorbiert oder reflektiert und letztlich das zurückkehrende, abgeschwächte Licht vom Gerät detektiert (Kämmerer 2009, Wally und Velik-Salchner 2015). Da die Lichtwellen vom Fettgewebe absorbiert und von der fettfreien Masse reflektiert werden, können anhand der gemessenen Restintensität des NIR-Lichtes der Körperfettanteil und die damit in Verbindung stehenden anderen Körperkompartimente bestimmt werden (DCK med GmbH, Dieterle et al. 2003).

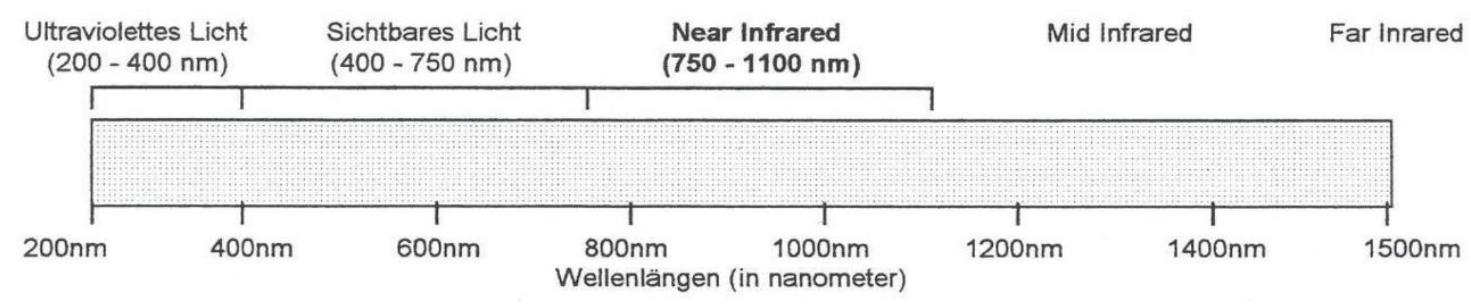

Abbildung 24: Lichtspektrum

(Das normale, sichtbare Licht mit einer Wellenlänge von 400 - $750 \mathrm{~nm}$ wird beim Durchtritt durch biologisches Gewebe fast vollständig absorbiert. Im sog. bio-optischen Fenster von 750 $950 \mathrm{~nm}$ weist Gewebe dagegen eine gute Transmission auf und das NIR-Licht kann eine Eindringtiefe von mehreren Zentimetern erreichen, sodass nicht nur Haut, sondern auch Muskelund Knochengewebe relativ ungehindert durchdrungen werden können (Haux 2007, Hirth 1999, Knauer 2009) (Die Verwendung der Abbildung erfolgt mit freundlicher Genehmigung der DCK med $\mathrm{GmbH}$ ) 
Physikalische Grundlage der Spektroskopie, also der Wechselwirkung zwischen Licht und Materie (GIT-Labor 2013), bildet das Lambert-Beer'sche Gesetz:

$$
A=\lg (10 / I)=E * C * d
$$

A= Abschwächung des Lichts; 10= Lichtintensität zu Beginn der Messung; I= Intensität des transmittierten Lichts; E= Extinktionskoeffizient; c= Stoffmengenkonzentration der absorbierenden Substanz; d= Schichtdicke des durchstrahlten Gewebes (Geisler 2013, Wikipedia)

Es beschreibt die Abschwächung der Intensität einer Strahlung beim Durchgang durch ein Medium in Abhängigkeit von dessen Konzentration und Schichtdicke (Wikipedia). Um den Lichtverlust durch Streuung nicht zu vernachlässigen, muss das Gesetz modifiziert und um einen korrigierenden Pfadlängenfaktor DPF und eine Konstante $G$ erweitert werden (Knauer 2009, Wally und Velik-Salchner 2015):

$$
A=E * c * d * D P F+G
$$

Da im Rahmen dieser Studie ein Gerät der Firma FUTREX verwendet wurde, sollen die o. g. Ausführungen darauf übertragen werden.

Mittels Infrarotdioden sendet die Messoptik zweimalig IR-Licht (Spektrum zwischen $920-950 \mathrm{~nm}$ ) ins Gewebe aus und nach gewebespezifischer Reflektion, Absorption und Streuung wird die verbleibende Restintensität des Lichts über ein Silizium-Sensor gemessen (Schlattmann 2005).

Als Messort wurde in der Vergangenheit standardmäßig der Biceps des dominanten Armes definiert. Dies rührt zum einen daher, dass sich die Ergebnisse bei Messungen an fünf verschiedenen Körperstellen im Vergleich zu nur einer Messung am Biceps nicht nennenswert voneinander unterschieden, und zum anderen auch daher, dass die Messergebnisse am Biceps mit denen von Referenzmethoden (beispielsweise Unterwasserwiege-Methode) am besten korrelierten.

Daraus lässt sich schließlich ableiten, dass die Kombination aus Hautdicke sowie Dicke und Zusammensetzung des Unterhautfettgewebes am Biceps optimale Bedingungen für die Durchdringung und Wechselwirkung mit NIR-Licht bieten muss und unter Einbeziehung von Körpergröße, Geschlecht und Gewicht eine direkte Korrelation zwi- 
schen diesem Messort und dem Gesamtkörperfettanteil besteht (Kalantar-Zadeh et al. 2006, Micro-Medical GmbH Rosenthal 1991).

Ausgehend vom Körperfettanteil, der dann auch als FM in kg angegeben wird, werden schließlich die FFM und das TBW berechnet (Schlattmann 2005).

\subsubsection{Spiroergometrie}

Da aus ihnen erweiterte diagnostische Erkenntnisse zu gewinnen sind und sie sowohl präventive als auch therapeutische Folgerungen zulassen, sind Belastungsuntersuchungen wie die Spiroergometrie aus Praxis und Klinik nicht mehr wegzudenken.

Die Spiroergometrie ist sowohl zur Beschreibung von Leistungsgrenzen und Leistungsreserven hilfreich, als auch für den Ausschluss gesundheitlicher Risiken von enormer Wichtigkeit (Kroidl et al. 2010). V. a. in der Sport- und Rehabilitationsmedizin ist sie eine wichtige diagnostische Methode zur Beurteilung der körperlichen Leistungsfähigkeit.

Die Spiroergometrie stellt das Ergebnis Generationen-überschreitender Forschungen auf den Gebieten Physik und Ingenieurswesen, Physiologie und Biochemie in Verbindung mit der klinischen Erfahrung dar und kann durch die Entwicklung der Computertechnik digital dargestellt werden (Kroidl et al. 2007).

„Die ersten Schritte in Richtung einer Belastungs-Physiologie liegen [allerdings] im Dunkeln“ (Kroidl et al. 2007, S. 291).

Weltweit das erste Fahrradergometer wurde vermutlich 1896 in Paris vom Franzosen Bouny entwickelt, während in Deutschland erst Mitte/Ende der 1920er Jahre die ersten Messungen im Sinne einer Spiroergometrie mittels Handkurbelergometer, kombiniert mit einem Spirographen, durchgeführt wurden (siehe Abbildung 25). 


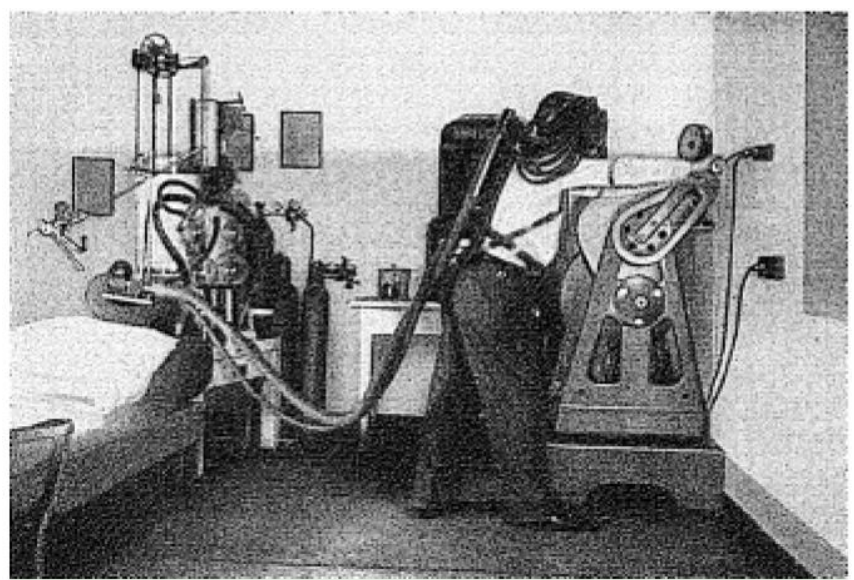

Die Leitungen zum Patienten sind verlängert und der Patient kann sich frei bewegen. Der Patient ist nicht durch ein Mundstück, sondern durch eine große, weiche Zelluloidmaske angeschlossen. Der Patient arbeitet am Ergographen Drehkurbelarbeit.

Abbildung 25: Erstes Handkurbelergometer (Die Verwendung der Abbildung erfolgt mit freundlicher Genehmigung von Dr. Rolf F. Kroidl-Stade und Prof. W. Hollmann)

In den 40er und 50er Jahren wurde die Ergometer-Technik so optimiert, dass Messsysteme zur Erfassung hoher Leistungen installiert werden konnten und somit die Grundlage für die Beschreibung der Beziehung von Ventilation und O2-Aufnahme und des Laktat-Stoffwechsels bildeten.

Heutzutage stehen Gas-Analysatoren zur Verfügung, die mit einer Analysezeit von 90 msec eine Erfassung physiologischer Daten Atemzug für Atemzug (breath by breath) ermöglichen. Zusammen mit Kreislauf- und Ventilationsparametern kommt so über eine Belastungsdauer von bis zu 15 Minuten eine enorme Datenmenge zusammen, die dank Computer-unterstützter Datenaufbereitung und schneller Drucker farbig und übersichtlich zusammengefasst dargestellt werden können (Kroidl et al. 2007).

Betrachtet man die Bestandteile des Wortes „Spiro-ergo-metrie" einmal einzeln, so lassen sich drei Bedeutungen daraus ableiten, die dann zusammen genau das beschreiben, was bei dieser Methode eigentlich gemessen wird.

„Spiro" steht für Atmung, „ergo" steht für Arbeit und „metrie" für Messung. Das bedeutet, dass die Leistung der Arbeit unter Belastung gemessen wird.

Wichtig dabei ist nur nicht zu vergessen, dass die Arbeit nicht alleine durch den Atemtrakt geleistet wird, sondern dass noch andere Systeme des Körpers wie beispielsweise das Herz-Kreislauf-System, die Muskulatur, das Nervensystem und der Stoffwechsel ebenfalls maßgeblich daran beteiligt sind. Kurz gesagt, wird mit der Spiroergometrie das Zusammenspiel all dieser Komponenten erfasst (Kroidl et al. 2010). 
Aus der Fülle an potenziell bestimmbaren Parametern musste für die Patientinnen der KOLIBRI-Studie zur Wahrung der Übersichtlichkeit eine gewisse Auswahl getroffen werden.

Als Entscheidungsgrundlage hierfür diente ein Artikel von Stöggl und Sperlich (2014) in dem beschrieben wird, dass in der Vergangenheit in vielen Ausdauersportarten immer wieder 5 Schlüsselparameter als Maßstab für die intra- und interindividuelle Vergleichbarkeit der sportlichen Leistungsfähigkeit herangezogen wurden.

Im Einzelnen sind dies die die VO2max bzw. VO2peak ( $\triangleq$ maximale Sauerstoffaufnahme), die work economy (^ VO2max bzw. VO2peak an der ventilatorischen Schwelle VT2), die peak power output (^ maximale Last bzw. Leistung), die power output at the lactate threshold ( $\hat{=}$ maximale Last an der Laktatschwelle (hier: Last an der ventilatorischen Schwelle VT2)) und die time to exhaustion ( $\triangleq$ Zeit bis zur Ausbelastung (hier: da einige Patientinnen die Ausbelastungsgrenze nicht erreichten, wurde zur besseren Vergleichbarkeit die Zeit bis zum Ende der Belastung verwendet)).

\section{VO2max bzw. VO2peak}

Die VO2max stellt den klassischen und wohl bekanntesten Kennwert zur Beurteilung der körperlichen Ausdauerleistungsfähigkeit dar. Er repräsentiert die Sauerstofftransportkapazität von Lunge und Herz-Kreislauf-System sowie den Sauerstoffverbrauch der beanspruchten Muskeln unter Belastung (Kroidl et al. 2010, Scharhag-Rosenberger und Schommer 2013) und gilt deshalb auch als „[...]wichtigster Index der aeroben Leistungsfähigkeit und der kardiorespiratorischen Funktion“ (Schöffl und Emmler, S. 3).

Nimmt man es ganz genau, kann eigentlich nur von VO2max gesprochen werden, wenn die maximale Ausbelastung erreicht und für eine gewisse Zeit durchgehalten wurde (Balady et al. 2010, Scharhag-Rosenberger und Schommer 2013). Weil das Erreichen der maximalen Ausbelastung schwierig zu definieren ist, sehr unterschiedlich empfunden wird und bei kardiovaskulär oder pulmonal vorerkrankten Patienten (hier auch bei Brustkrebspatientinnen) nur selten zu beobachten ist, wird häufig auch der Begriff VO2peak - also die Sauerstoffaufnahme bei Abbruch der Belastung - verwendet (Balady et al. 2010). Die Normalwerte für VO2max bzw. VO2peak sind geschlechtsabhängig und nehmen mit zunehmendem Alter ab (Wonisch et al. 2003). 
Aufgrund der höheren klinischen Relevanz und der besseren interindividuellen Vergleichbarkeit (Breuer 2004) wird hier die auf das Körpergewicht bezogene Sauerstoffaufnahme $\mathrm{VO} / \mathrm{kg}$ verwendet.

\section{$\underline{\mathrm{VO} 2 / \mathrm{kg}}$}

Die VO2/kg - auch als relative VO2 bezeichnet - wird u. a. zur Charakterisierung der Fitness, zur Risikoabschätzung und als Prognose-Indikator genutzt (Kroidl et al. 2010). Bei nicht ausdauertrainierten gesunden 30-jährigen Frauen und Männern beträgt die relative VO2 zwischen $35-50 \mathrm{ml} / \mathrm{min} / \mathrm{kg}$. Je nach Fitnesszustand bzw. Vorerkrankung kann es jedoch erhebliche Abweichungen hiervon geben.

So können hochtrainierte Ausdauerathleten Werte bis $90 \mathrm{ml} / \mathrm{min} / \mathrm{kg}$ erreichen, während herzinsuffiziente Patienten (bis NYHA III) oder Patientinnen mit Brustkrebs während oder unmittelbar nach der Chemotherapie nur im Bereich zwischen 15 und $20 \mathrm{ml} / \mathrm{min} / \mathrm{kg}$ liegen können.

Generell ist anzumerken, dass die Werte bei Frauen im Vergleich zu Männern aufgrund ihrer geringeren Muskelmasse etwa 10 - 15\% niedriger liegen und dass sie im Allgemeinen ab dem 30. Lebensjahr um etwa 8 -10\% pro Dekade abnehmen (ScharhagRosenberger und Schommer 2013).

\section{$\underline{\text { Last }}$}

Die Last bzw. Leistung wird in der Einheit Watt angegeben und beschreibt den Energieumsatz pro Zeiteinheit (Wikipedia).

Die meisten Watt-Sollwerte werden ebenfalls gewichts- und altersbezogen angegeben. Dies hat zur Folge, dass mit zunehmendem Gewicht die vorausgesagte Leistung steigt, obwohl ja eigentlich von mehr Körpergewicht nicht automatisch auf mehr Muskelmasse geschlossen werden kann. Von den Autoren des Kursbuch Spiroergometrie wird die Tabelle des American College of Sports Medicine (ACSM) empfohlen. Die hier angegebenen Sollwerte haben sich in der Praxis vielfach bewährt, da sie die Verhältnisse einer Normalbevölkerung gut abbilden (Kroidl et al. 2010).

\section{$\underline{\text { RER - Respiratory exchange rate/ratio }}$}

Sie spiegelt das Verhältnis aus VCO2/VO2 wider (= pro Zeiteinheit abgeatmete Menge $\mathrm{CO} 2$ geteilt durch die in der gleichen Zeiteinheit eingeatmete Menge O2). 
In der Physiologie der Atmung wird dieses Verhältnis als Respiratorischer Quotient (RQ) oder auch Stoffwechsel-RQ bezeichnet. Demgegenüber steht der in der Sprache der Spiroergometrie verwendete Begriff Lungen-RQ (=RER).

Im Falle idealer Atmung und unter stabilen Bedingungen (steady state) entsprechen sich der Lungen- und der Stoffwechsel-RQ. Dann hängt die RER vom metabolischen Substrat der Energiegewinnung ab, weshalb dann der Anteil der Fett- bzw. Kohlenhydratverwertung abgeschätzt werden kann (Kroidl et al. 2010, Wonisch et al. 2003). Bei reiner Fettverbrennung beträgt der $\operatorname{RER}=0,7$, bei reiner Kohlenhydratverbrennung $\mathrm{RER}=1$ und bei reiner Proteinverbrennung theoretisch $\mathrm{RER}=0,8$. In der Regel liegen allerdings Mischverhältnisse zwischen Fett- und Kohlenhydratverbrennung vor, sodass sich ein RER von 0,8 eher aus diesen beiden Anteilen zusammensetzt, als aus reiner Proteinverbrennung. Diese stellt abseits von Hungerperioden oder Dauerbelastungen von mehr als 60 Minuten allerdings keine zu berücksichtigende Einflussgröße dar (Meyer 2003). Während in Ruhe vorwiegend Fettverbrennung stattfindet, nimmt der Anteil der Kohlenhydratverbrennung an der Energiegewinnung mit steigender Intensität der Belastung zu.

Die Rückschlüsse von der RER auf die verstoffwechselten Substrate sind allerdings nur bis zum sog. aerob-anaeroben Übergang möglich. Bei noch weiter ansteigender Belastung gewinnt das sog. ExcessCO2 aus der Laktatpufferung zunehmenden Einfluss auf die RER (Kroidl et al. 2010) (siehe Abbildung 26). Milchsäure $\rightarrow$ Laktat $+\mathrm{H}^{+}$ $\mathrm{HCO}_{3}{ }^{-}+\mathrm{H}^{+} \rightarrow \mathrm{H}_{2} \mathrm{O}+\underline{\mathrm{CO}_{2}}$

Abbildung 26: Entstehung von Excess-CO2 (Die Verwendung der Abbildung erfolgt mit freundlicher Genehmigung von Prof. Dr. med Durch die Pufferung der bei der Laktatbildung anfal- Tim Meyer) lenden Protonen durch Bicarbonat entsteht zusätzliches Kohlenstoffdioxid (= ExcessCO2), das abgeatmet werden muss und somit die RER deutlich erhöht. Als Folge können somit RER-Werte > 1,2 erreicht werden (Meyer 2003).

\section{Ventilatorische Schwellen}

Als ventilatorische Antwort auf die Stoffwechselvorgänge unter zunehmender Belastung werden zwei ventilatorische Schwellen definiert. Diese kennzeichnen den Beginn und das Ende des aerob-anaeroben Übergangsbereiches, der individuell unterschiedlich lang sein kann (Westhoff et al.). 
Aufgrund der vielen und damit unübersichtlichen Terminologien für die beiden Schwellen werden sie in dieser Arbeit genau wie im Positionspapier der AG-Spirergometrie von 2012 als VT1 (= ventilatory threshold 1) und VT2 (=ventilatory threshold 2) bezeichnet.

Die VT1 ist gekennzeichnet durch eine Steigerung der Ventilation und der CO2-Abgabe (VCO2) im Verhältnis zur Sauerstoffaufnahme (VO2) aufgrund des anfallenden ExcessCO2 (Westhoff et al.).

Bei Ausdauersportlern liegt sie i.d.R. zwischen 60 - 70\% der VO2max bzw. bei Untrainierten zwischen 45 - 60\% der VO2max (Scharhag-Rosenberger und Schommer 2013).

Die VT2 ist dagegen dann erreicht, wenn es aufgrund der zunehmenden metabolischen Azidose zu einer nochmaligen und überproportionalen Steigerung der Ventilation kommt (Westhoff et al.). Sie wird auch als Respiratorischer Kompensationspunkt bezeichnet und ist als RER $\geq 1$ definiert (Kroidl et al. 2010).

Die Referenzwerte liegen hier etwa zwischen 80 - 90\% der VO2max bei Ausdauerleistungssportlern bzw. zwischen $70-80 \%$ der VO2max bei Untrainierten (ScharhagRosenberger und Schommer 2013).

\section{Laktatschwellen}

Wenn der Energiebedarf der Muskulatur nicht mehr durch den oxidativen Abbau von Fetten und Kohlenhydraten gedeckt werden kann, muss Energie zusätzlich durch anaerobe Glykolyse bereitgestellt werden. Endprodukt hierbei ist Laktat, das Salz der Milchsäure.

Eine Akkumulation von Laktat in den Muskelzellen führt zur fortschreitenden metabolischen Azidose und schließlich zur Hemmung des Energiestoffwechsels. Aus diesem Grund ist die Bestimmung des Laktatwertes heutzutage fester Bestandteil der Spiroergometrie (Kroidl et al. 2010).

Historisch werden zwei Laktatschwellen beschrieben, die prinzipiell den Beginn und das Ende des aerob-anaeroben Übergangs betreffen. Die erste Schwelle spielt bisher im Rahmen der Laktatdiagnostik eine untergeordnete Rolle und bildet den ersten Anstieg der Laktatkonzentration ab, die zweite Schwelle ist sicherlich bekannter (meist bei $4 \mathrm{mmol} / \mathrm{l}$ angegeben) und ist Ausdruck des Endes der aerob-anaeroben Schwelle. 
Zur besseren Berücksichtigung individueller Unterschiede und Beurteilung der Laktatkinetik, werden heute eher individuelle Schwellen propagiert und die Entwicklung geht weg vom reinen Laktatschwellenkonzept hin zu einem Konzept der Kombination ventilatorischer und metabolischer (Laktat-) Parameter (Westhoff et al. 2012).

In dieser Arbeit wurde der Laktatwert 3 Minuten nach Belastungsende bestimmt.

\subsection{Durchführung der Messungen}

Die Durchführung der Messungen zur Erfassung der Körperzusammensetzung erfolgte an den Tagen Montag bis Freitag jeweils zwischen 11 und 12 Uhr vormittags jeweils in folgender Reihenfolge: Dualenergie-Röntgen-Absorptiometrie, bioelektrische Impedanzanalyse, Körperfettwaage und Nahinfrarot-Spektroskopie.

Somit wurde bei allen Patientinnen in etwa zur gleichen Tageszeit gemessen, wodurch eventuelle Schwankungen der Ergebnisse durch unterschiedliche Tageszeiten und Tagesrhythmik auf ein Minimum reduziert werden konnten und die Messergebnisse diesbezüglich vergleichbar sind.

Um außerdem bestmögliche Voraussetzungen für die Vergleichbarkeit der Ergebnisse zu schaffen, sollte das Frühstück am Morgen zwischen 7 und $8 \mathrm{Uhr}$ eingenommen werden. Danach durfte bis zum Messbeginn nichts mehr gegessen werden, wodurch gewährleistet war, dass die Patientinnen ca. 3 - 4 h nüchtern waren. Wasser, Tee, Kaffee oder sonstige Getränke durften wie gewohnt getrunken werden.

Wenn möglich, sollten die Patientinnen kurz vor den Messungen noch zur Toilette gehen, um ihre Blase zu entleeren (v. a. wichtig für die BIA und Körperfettwaage).

Des Weiteren sollten sich die Patientinnen morgens vor der Messung nicht mehr sportlich betätigen. Dies hätte v. a. bei den Ergebnissen der BIA und Körperfettwaage Abweichungen zur Folge gehabt.

Dauermedikamente - sofern verschrieben - durften selbstverständlich nach Plan eingenommen werden.

Vor Beginn der Messungen wurde standardisiert das Körpergewicht mittels Personenwaage (Modell 920 von seca) und die Körpergröße mittels an der Wand montierten Maßbands erfasst. Dafür mussten Kleidung, Schuhe und Schmuck ausgezogen bzw. 
abgelegt werden.

Das auf diese Weise erfasste Körpergewicht wurde für die BIA- und FUTREX-Messung verwendet. Das DXA-Gerät und die Körperfettwaage hatten eine integrierte Waage, sodass hier mit leicht abweichenden Werten gerechnet wurde.

Da die Patientinnen bei der Dualenergie-Röntgen-Absorptiometrie geringer Strahlung und bei der bioelektrischen Impedanzanalyse und Körperfettwaage geringem Wechselstrom ausgesetzt waren und sich die Literatur noch sehr uneinig darüber ist, welche Gefahr hierdurch für Schwangere bzw. das noch ungeborene Kind ausgeht, wurde sicherheitshalber vor Beginn der Messungen nochmals eine mögliche Schwangerschaft anamnestisch ausgeschlossen (obwohl Schwangerschaft bereits ein Ausschlusskriterium für die Teilnahme an der Studie war). In diesem Fall wären die Messungen DXA, BIA und Körperfettwaage nicht durchgeführt worden.

Alle weiteren für die jeweilige Methode spezifischen Ausschlusskriterien bzw. Standard-Untersuchungsbedingungen werden im Folgenden bei den entsprechenden Messmethoden erläutert. Falls nicht anders gekennzeichnet, stammen alle Informationen und Abbildungen zu den Messmethoden und deren Durchführung entweder aus den entsprechend mitgelieferten Bedienungs- oder Kurzanleitungen oder wurden im Rahmen einer Herstellereinweisung vermittelt.

\subsubsection{Dualenergie-Röntgen-Absorptiometrie}

Für die Messung der Körperzusammensetzung mittels DXA wurde das Modell Explorer (S/N 90425) der Firma HOLOGIC verwendet (siehe Abbildung 27).

Wichtigste Bestandteile sind der Untersuchungstisch, auf dem die Patientinnen gelagert wurden, der sog. C-Arm, der im unteren Teil die Strahlungsquelle und im oberen, gegenüberliegenden Teil die entsprechenden Detektoren besitzt und schließlich die Computereinheit, an die die Roh-Messdaten gesendet, mittels entsprechender Software (hier QDR for Windows XP) in Bilder und mittels integriertem Algorithmus in errechnete Werte zur Zusammensetzung der einzelnen Körperkompartimente umgewandelt wurden. 


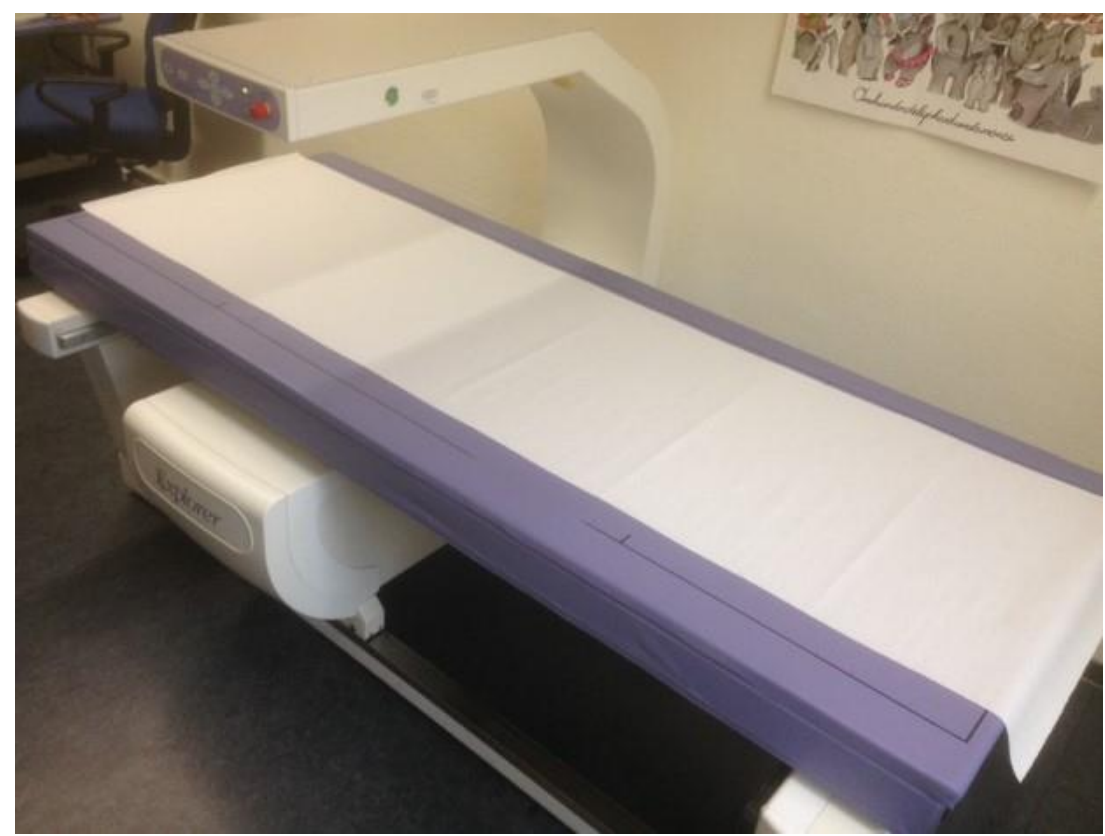

Abbildung 27: In der Studie verwendetes (Original-) DXA-Gerät

Vor der ersten Messung eines jeden Tages musste mit Hilfe eines Wirbelsäulen-Modells (siehe Abbildung 28) ein etwa 2-minütiger automatischer Systemtest durchgeführt werden. Erst nach erfolgreich bestandenem Test hätte mit der Knochendichtemessung begonnen werden können.

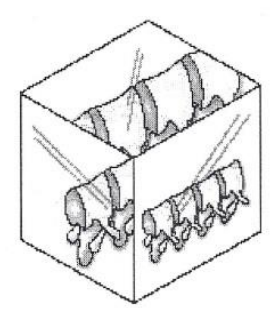

Abbildung 28:

Wirbelsäulen-

Modell

musste zusätzlich einmal wöchentlich eine Kalibrierung mittels Stufen-Modell durchgeführt werden.

In Abbildung 29 ist die geforderte genaue Platzierung des Modells zu sehen.

Wurde auch dieser Test erfolgreich bestanden, musste das Stufen-Modell entfernt und zu guter Letzt ein automatischer Test für die radiographische Einheitlichkeit durchgeführt werden. Erst dann konnte mit den weiteren Vorbereitungen für die eigentliche Messung in der jeweiligen Woche fortgefahren werden. 


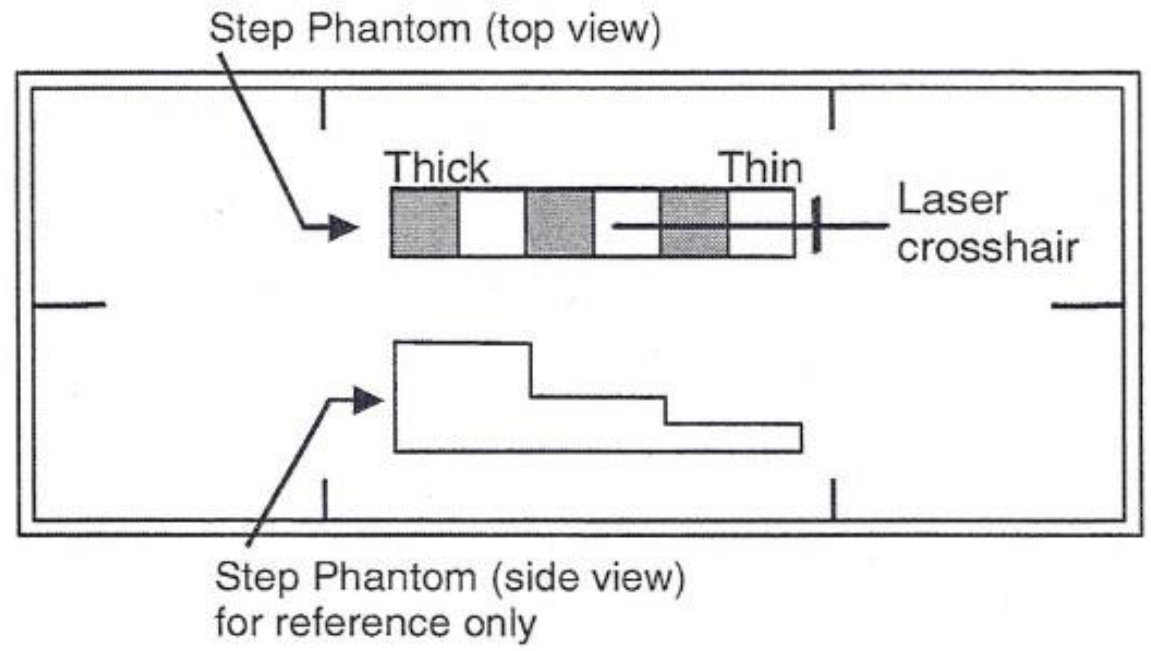

Abbildung 29: Stufen-Modell

Im Folgenden sind einige Vorschläge des Herstellers für Fragen aufgeführt, die vor Beginn der Messung zusätzlich abgeklärt werden sollten:

- Gab es in letzter Zeit irgendwelche nuklearmedizinischen Untersuchungen? (wenn ja, hätte mit der entsprechenden Nuklearmedizinischen Abteilung Rücksprache gehalten werden müssen).

- Ist Fremdmaterial im Körper vorhanden? (z. B. Schrittmacherdrähte, MetallImplantate, Klammernähte, Granatsplitter o. ä.).

- Sind Piercings vorhanden? (hätten entfernt werden müssen).

Nachdem anschließend die Patientendaten (Name, Vorname, Geburtsdatum, Geschlecht, Ethnizität, Körpergröße und gewicht) eingetragen und der Scan-Typ whole body ausgewählt wurde, konnte mit der Positionierung der Patientinnen auf dem Untersuchungstisch fortgefahren werden (siehe Abbildung 30).

Die maximale Scan-Länge betrug $195 \mathrm{~cm}$, die maximale Scan-Breite $69 \mathrm{~cm}$ und die maximale Tragkraft des Untersuchungstisches lag bei 350 pounds (= ca. $160 \mathrm{~kg}$ ).

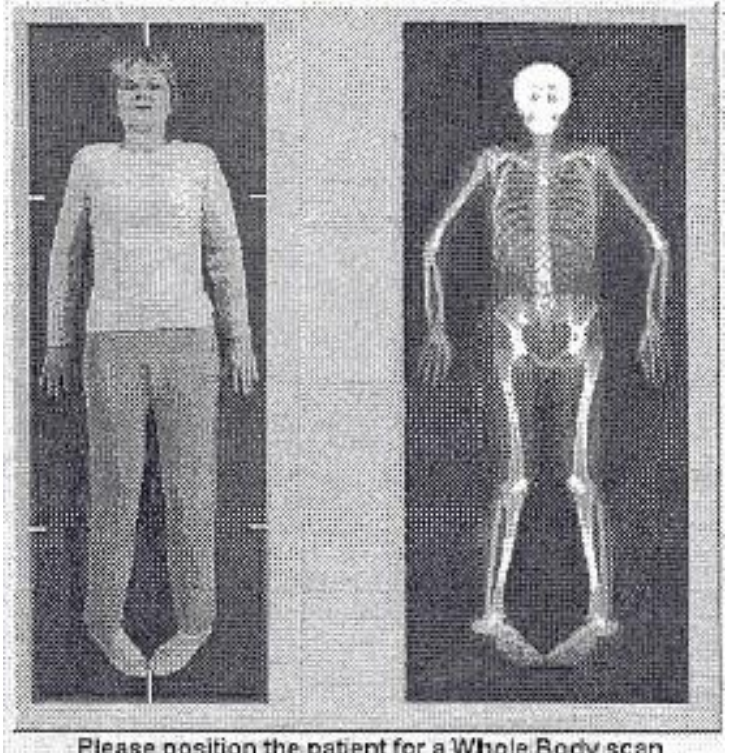

Please position the patient for a Whole Bocly scan Abbildung 30: Korrekte Positionierung 
Folgende Vorgaben zur Positionierung wurden vom Hersteller gemacht:

- Flach auf dem Rücken liegen mit Blick an die Decke.

- Der gesamte Körper inklusive Hände und Füße sollten sich innerhalb der Markierungslinien und damit im Scan-Feld befinden.

- Arme an die Seite des Körperstammes legen, ohne diesen oder die Oberschenkel zu berühren.

- Handflächen nach unten.

- Beine etwa $25^{\circ}$ nach Innen rotieren, sodass sich die Zehen berühren.

- In dieser Position ruhig liegen bleiben, nicht sprechen, normal atmen.

Anschließend konnte die etwa 7-minütige Messung gestartet werden. Die dabei verwendete Strahlung war so gering, sodass sich der Untersucher / die Untersucherin die ganze Zeit im selben Raum aufhalten konnte (Pandey et al. 2011).

Am entstandenen Bild konnten nun noch der Kontrast und die Helligkeit verändert werden (ohne Einfluss auf die Berechnungen) und es mussten noch die entsprechenden Regionen (ROI= region of interest) für die weiteren Berechnungen ausgewählt und angepasst werden (siehe Abbildung 31).

Folgende Anpassungen wurden im sog. Line Mode durchgeführt:

- Die horizontalen Linien mussten von oben nach unten unterhalb des Kiefers bzw. etwa auf Höhe Th12 - L1 bzw. gerade oberhalb des Beckenkamms platziert werden.

- Die vertikalen Linien mussten so nah wie möglich beidseits an die Brust bzw. Wirbelsäule bzw. an die Beine und genau zwischen die Beine platziert werden.

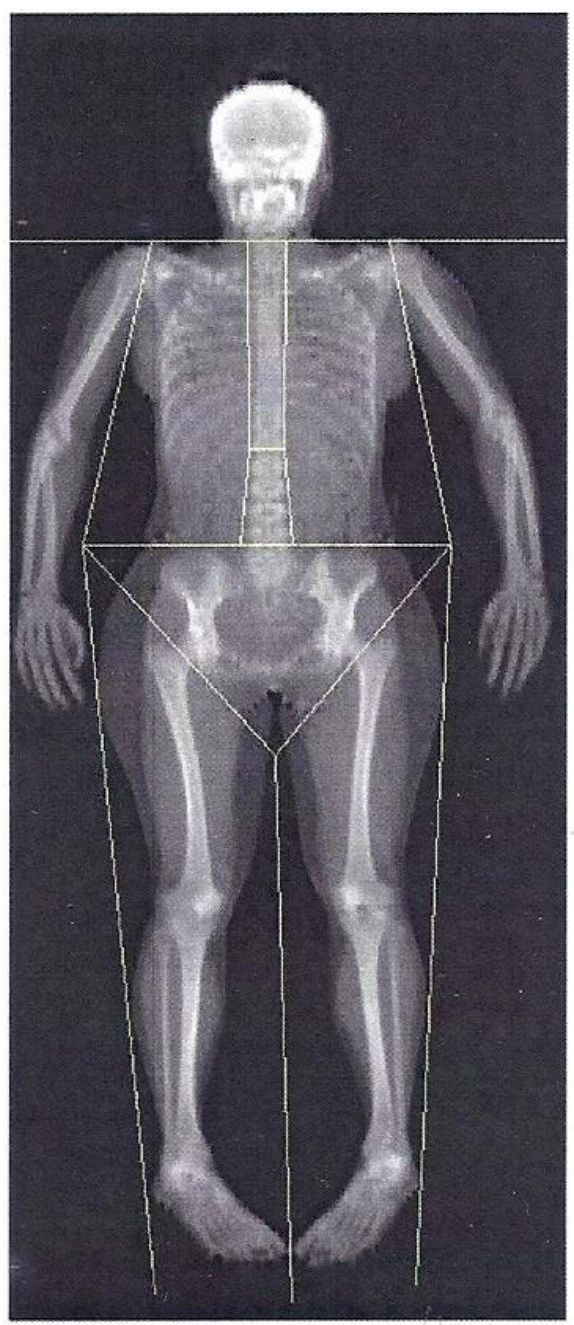

Abbildung 31: Anpassungen im Lineund Point-Mode 
Für noch genauere Anpassungen stand der sog. Point Mode zur Verfügung. In diesem Modus konnten die Punkte, die an den Schnittstellen der vertikalen und horizontalen Linien entstanden, noch entsprechend verschoben werden:

- Die Punkte im Bereich der Schultern sollten so verschoben werden, dass sie sich jeweils knapp oberhalb des Bereiches zwischen Oberarmkopf und Schultergelenkpfanne befanden.

- Die Punkte im Bereich der Wirbelsäule sollten so nah wie möglich an die Wirbelsäule platziert werden.

- Die Punkte im Bereich der Hüften sollten soweit nach außen verschoben werden, dass das Weichgewebe der Hüften und Oberschenkel eingeschlossen wurde.

- Der Punkt zwischen den Beinen (unterer Punkt des Dreiecks) sollte genau zwischen die beiden Oberschenkel platziert werden.

- Die Punkte an den Beinen sollten so platziert werden, dass möglichst viel Weichgewebe der Beine eingeschlossen wurde. Dabei musste darauf geachtet werden, dass dadurch die Hände und Finger nicht eingeschlossen wurden.

Anschließend konnte die Berechnung abgeschlossen und die Ergebnisse angezeigt und ausgedruckt werden. Auf dem Ausdruck waren dann neben allgemeinen Messinformationen und der oben bereits gezeigten Abbildung 31 noch folgende Werte ersichtlich (siehe Abbildungen 32 - 34):

\begin{tabular}{|lrrr|}
\hline Bereich & $\begin{array}{r}\text { Fläche } \\
\left(\mathbf{c m}^{2}\right)\end{array}$ & $\begin{array}{c}\text { BMC } \\
(\mathbf{g})\end{array}$ & $\begin{array}{r}\text { BMD } \\
\left(\mathbf{g} / \mathbf{c m}^{2}\right)\end{array}$ \\
L Arm & 166.96 & 116.43 & 0.697 \\
R Arm & 195.84 & 141.54 & 0.723 \\
L Ribs & 158.65 & 102.30 & 0.645 \\
R Ribs & 146.78 & 96.68 & 0.659 \\
T Spine & 117.90 & 102.47 & 0.869 \\
L Spine & 60.53 & 59.36 & 0.981 \\
Becken & 226.30 & 270.16 & 1.194 \\
L Leg & 325.61 & 362.14 & 1.112 \\
R Leg & 333.12 & 369.59 & 1.109 \\
T.-Summe731.68 & 1620.67 & 0.936 \\
Kopf & 250.83 & 557.23 & 2.222 \\
Gesamt & $\mathbf{1 9 8 2 . 5 1}$ & $\mathbf{2 1 7 7 . 9 0}$ & $\mathbf{1 . 0 9 9}$ \\
\hline
\end{tabular}

Abbildung 32: Ergebnisse für BMC und BMD für die einzelnen Körperregionen

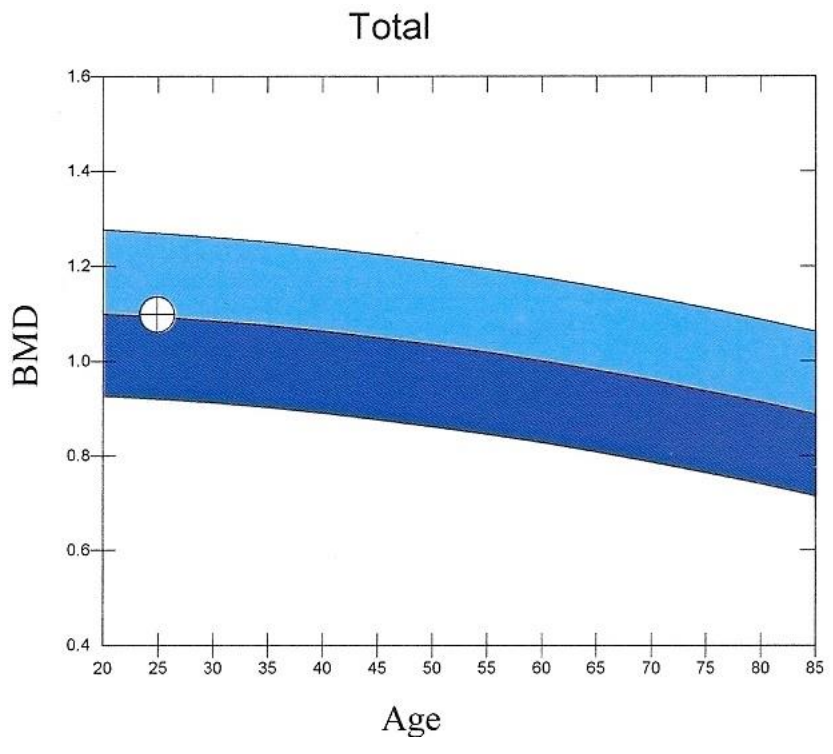

Abbildung 33: Normbereich der BMD bezogen auf das Alter 
DXA Results Summary:

\begin{tabular}{|lrrrrrr|}
\hline Bereich & $\begin{array}{r}\text { BMC } \\
(\mathbf{g})\end{array}$ & $\begin{array}{r}\text { Fett } \\
(\mathbf{g})\end{array}$ & $\begin{array}{r}\text { Muskel } \\
(\mathbf{g})\end{array}$ & $\begin{array}{r}\text { Muskel+BMC } \\
(\mathbf{g})\end{array}$ & $\begin{array}{r}\text { Gesamtmasse } \\
(\mathbf{g})\end{array}$ & $\begin{array}{r}\text { \%Lipide } \\
\text { L Arm }\end{array}$ \\
116.43 & 698.6 & 1765.0 & 1881.5 & 2580.1 & 27.1 \\
R Arm & 141.54 & 750.1 & 1938.6 & 2080.1 & 2830.2 & 26.5 \\
Rumpf & 630.97 & 7335.1 & 21861.1 & 22492.1 & 29827.2 & 24.6 \\
L Leg & 362.14 & 3609.3 & 6508.9 & 6871.0 & 10480.3 & 34.4 \\
R Leg & 369.59 & 3901.0 & 6725.2 & 7094.8 & 10995.8 & 35.5 \\
T.-Summe & 1620.67 & 16294.2 & 38798.8 & 40419.4 & 56713.6 & 28.7 \\
Kopf & 557.23 & 948.7 & 3281.9 & 3839.1 & 4787.9 & 19.8 \\
Gesamt & $\mathbf{2 1 7 7 . 9 0}$ & $\mathbf{1 7 2 4 2 . 9}$ & $\mathbf{4 2 0 8 0 . 6}$ & $\mathbf{4 4 2 5 8 . 5}$ & $\mathbf{6 1 5 0 1 . 5}$ & $\mathbf{2 8 . 0}$ \\
\hline
\end{tabular}

Abbildung 34: Zusammenfassung aller Messergebnisse (außer BMD)

\subsubsection{Bioelektrische Impedanzanalyse}

Für die bioelektrische Impedanzmessung wurde das Gerät Nutriguard-M (Body Impedance Analyzer) der Firma Data-Input $\mathrm{GmbH}$ verwendet (siehe Abbildung 35). Außerdem wichtig waren das entsprechende Messkabel, Spezialelektroden und ein PC mit Auswertungssoftware (hier ginal-) BIA-Gerät

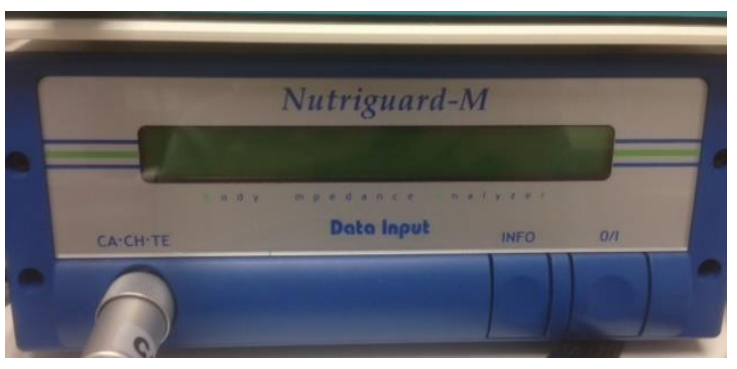

Abbildung 35: In der Studie verwendetes (OriFORANA Tethys V3.1)

Vor erstmaliger Messung sollte ein Geräte-, ein Kabel- und ein Elektrodentest durchgeführt werden. Beim Gerätetest mit Original-Teststecker sind bei $50 \mathrm{kHz}$ folgende Sollwerte vom Hersteller angegeben:

- Für die Resistance R: $500+/-4$

- Für die Reactance Xc: $144+/-4$

Beim Kabel-Test werden alle Messklemmen ineinander (oder an eine Münze) gesteckt und es sollten sowohl für R als auch für Xc als Messergebniss 0 (= Null) angezeigt werden.

Beim Elektroden- bzw. Sandwich-Test (siehe Abbildung 36) werden zwei Elektroden mit der Gelseite aneinandergeklebt, wobei die beiden Laschen in entgegengesetzte Richtungen zeigen sollen. Die rote und schwarze Klemme eines jeden Messkabels wird jeweils an einer Lasche befestigt. Wiederum beim $50 \mathrm{kHz}$ sollten $\mathrm{R}<30$ und $\mathrm{Xc}<1$ sein. 


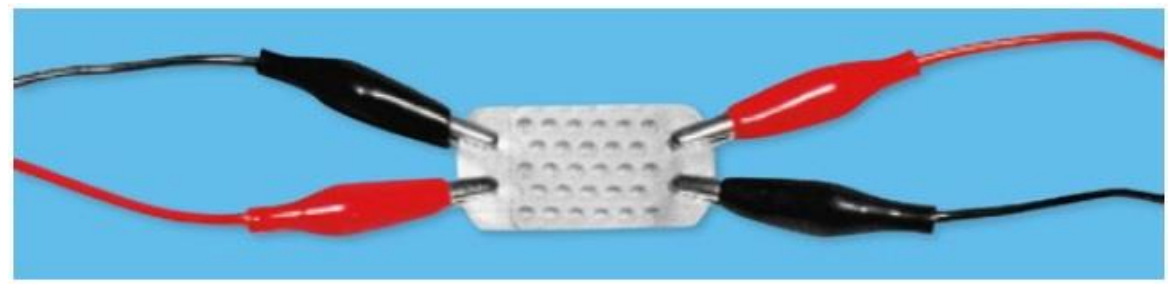

Abbildung 36: Elektroden- bzw. Sandwichtest

Die aussagekräftigsten Messergebnisse lassen sich bei völliger körperlicher Ruhe in flacher, horizontaler Rückenlage auf einer elektrisch isolierten Unterlage erzielen. Außerdem wäre eine einstündige Liegezeit vor der Durchführung der Messung ideal, da sich dadurch das Körperwasser gleichmäßig im Gesamtorganismus verteilt. Da dies aus praktischen Gründen kaum durchführbar ist, sollte die Messung nicht unmittelbar nach dem Hinlegen, sondern erst nach einer Wartezeit von wenigen Minuten erfolgen (Reimers et al. 2005).

Da die BIA-Messung erst nach der DXA-Messung durchgeführt wurde, war somit eine Liegezeit zwischen 7 - 10 Minuten gewährleistet. Auch die erforderliche flache Lagerung auf elektrisch isolierter Unterlage war auf dem Untersuchungstisch des DXAGerätes gegeben.

Weitere Standardbedingungen, die in der Literatur und der Kurzanleitung zum o. g. Gerät von Data Input für eine verlässliche und v. a. reproduzierbare Messung gefordert werden, wurden hier ebenfalls annähernd erfüllt:

- möglichst 4 - 5 Stunden vorher nüchtern.

- die letzte sportliche Betätigung sollte möglichst 12 Stunden zurückliegen.

- der letzte Alkoholkonsum sollte möglichst 24 Stunden zurückliegen.

„[D]a nicht mit Sicherheit auszuschließen ist, dass das bei der Messung induzierte Stromfeld den Defibrillator aktiviert" (Data Input 2015), war das Tragen eines Herzschrittmachers und/oder Defibrillators neben Schwangerschaft eine absolute Kontraindikation.

Bei der Lagerung der Patientinnen für die BIA-Messung war abweichend zur Lagerung bei der DXA-Messung folgendes zu beachten (siehe Abbildung 37): 
- Die Beine der Patientinnen wurden in einem Winkel von ca. $45^{\circ}$ gespreizt, sodass sich die Oberschenkel nicht berührten.

- Die Arme sollten ca. $30^{\circ}$ abgespreizt sein und durften keinen

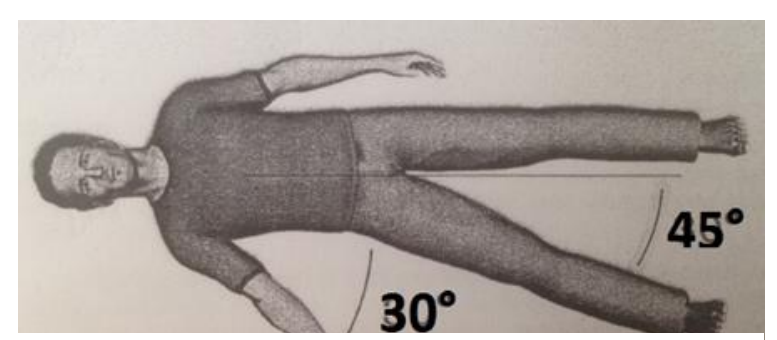

Abbildung 37: Lagerung bei der BIA Kontakt zu anderen Körperteilen oder Metallgegenständen haben.

- Aus Standardisierungsgründen erfolgte das Anbringen der Messelektroden immer auf der dominanten Körperseite. Dies wurde auch bei den Verlaufsmessungen berücksichtigt.

Da die Verwendung ungeeigneter Elektroden (hier wurden die Elektroden BIANOSTIC $A T$ in der Größe double size von Data Input verwendet) und deren falsche Platzierung zu den häufigsten Fehlerquellen gehören, wurde beim Aufkleben sehr genau auf folgende Vorgaben geachtet (siehe Abbildung 38):

- Bei den Hautarealen, auf die die Elektroden aufgeklebt wurden, wurde darauf geachtet, dass diese sauber und trocken waren.

- Grundsätzlich war darauf zu achten, dass an die distale (= körperferne) Elektrode die rote Klemme und an die proximale (= körpernahe) Elektrode die schwarze Klemme angeschlossen wurde.

- Die Kabel hingen dabei frei und nicht verdrillt in der Luft und hatten dabei keinen Kontakt zu Metallflächen.

- Die Handgelenkselektrode (= Messelektrode) wurde mit ihrem proximalen Ende an der gedachten Linie durch das Ulnaköpfchen angebracht. Die Fingerelektrode (= Signalelek-
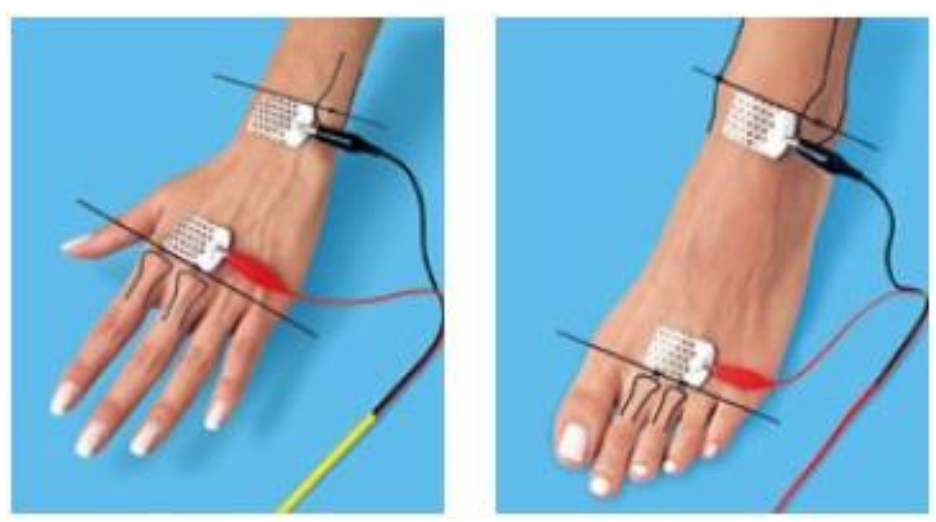

Abbildung 38: Elektrodenpositionierung trode) wurde mit ihrem distalen Rand entlang der gedachten Linie durch die Mitte der Fingergrundgelenke von Zeige- und Mittelfinger platziert. 
- Die Sprunggelenkselektrode (= Messelektrode) wurde mit ihrem proximalen Rand an die gedachte Linie durch Außen- und Innenknöchel geklebt. Die Zehenelektrode (= Signalelektrode) gehörte schließlich noch mit ihrem distalen Rand an die gedachte Linie durch die Mitte der Grundgelenke von 2. und 3. Zehe.

Der Abstand der Elektroden sollte so jeweils etwa $5 \mathrm{~cm}$ betragen. Bei kleinerem $\mathrm{Ab}$ stand bestand sonst die Gefahr der Elektroden-Interaktion.

Bevor die eigentliche Messung nun gestartet werden konnte, mussten die allgemeinen Patientendaten (Name, Vorname, Geburtsdatum, Geschlecht, Größe und Gewicht) noch am PC in die Maske des Auswertungsprogramms eingegeben werden.

Da es sich bei der Messung um eine Multifrequenz-Messung handelte, erfolgten 3 Messungen mit jeweils unterschiedlicher Frequenz. Die so direkt gemessenen und auf dem Display angezeigten Werte für die Resistance $R$ und die Reactance Xc bei 5,50 und $100 \mathrm{kHz}$ (siehe Abbildung 39) mussten extra notiert und im Anschluss in eine weitere Eingabemaske des Programmes eingegeben werden.

\begin{tabular}{|lllllll|}
\hline Datum: & Freitag 15. November 2013 11:30 & Messzyklus: & 1 & Messung Nr: \\
5 & kHz & R 839 & Xc 339 & Geschlecht: & weiblich & \\
50 & kHz & R 738 & Xc 75 & GrôBe: & $158 \mathrm{~cm}$ \\
100 & kHz & R 701 & Xc 68 & Gewicht: & $55.0 \mathrm{~kg}$
\end{tabular}

Abbildung 39: Messergebnisse für $\mathrm{R}$ und $\mathrm{XC}$ bei 5,50 und $100 \mathrm{kHz}$

Dabei wurde bereits auf evtl. stark abweichende oder nicht nachvollziehbare Werte geachtet und ggf. eine Konsistenzprüfung der Rohwerte bei $50 \mathrm{kHz}$ durchgeführt:

- Der physiologische Bereich für R beträgt bei Frauen 400 - 750 Ohm, bei Männern 350 - 650 Ohm. Liegen die Messwerte für R außerhalb dieses Bereiches, liegt die Ursache in den seltensten Fällen in extrem kleinen oder großen Werten für die BCM oder in Ödemen im Körper. In den allermeisten Fällen sind solche Werte auf Probleme mit den Messkabeln oder Elektroden zurückzuführen.

- Der physiologische Bereich für Xc liegt zwischen 8-14\% des jeweiligen RWertes. Werte $<8 \%$ können bei Malnutrition auftreten, Werte $>14 \%$ dagegen 
beispielsweise bei Bodybuildern. Aber auch hier sind Probleme mit den Messkabeln oder Elektroden die häufigste Ursache für abweichende Werte.

Aus den Werten von R und Xc wurde anschließend die Zusammensetzung der einzelnen Körperkompartimente berechnet und wie in Abbildung 40 zu sehen dargestellt. Auf den Folgeseiten des Ergebnisausdrucks waren zusätzlich zu den einzelnen Messergebnissen BMI, Körperfett, BCM, ECM/BCM-Index und Phasenwinkel kurze Abschnitte mit Erklärungen und Interpretationen aufgeführt.

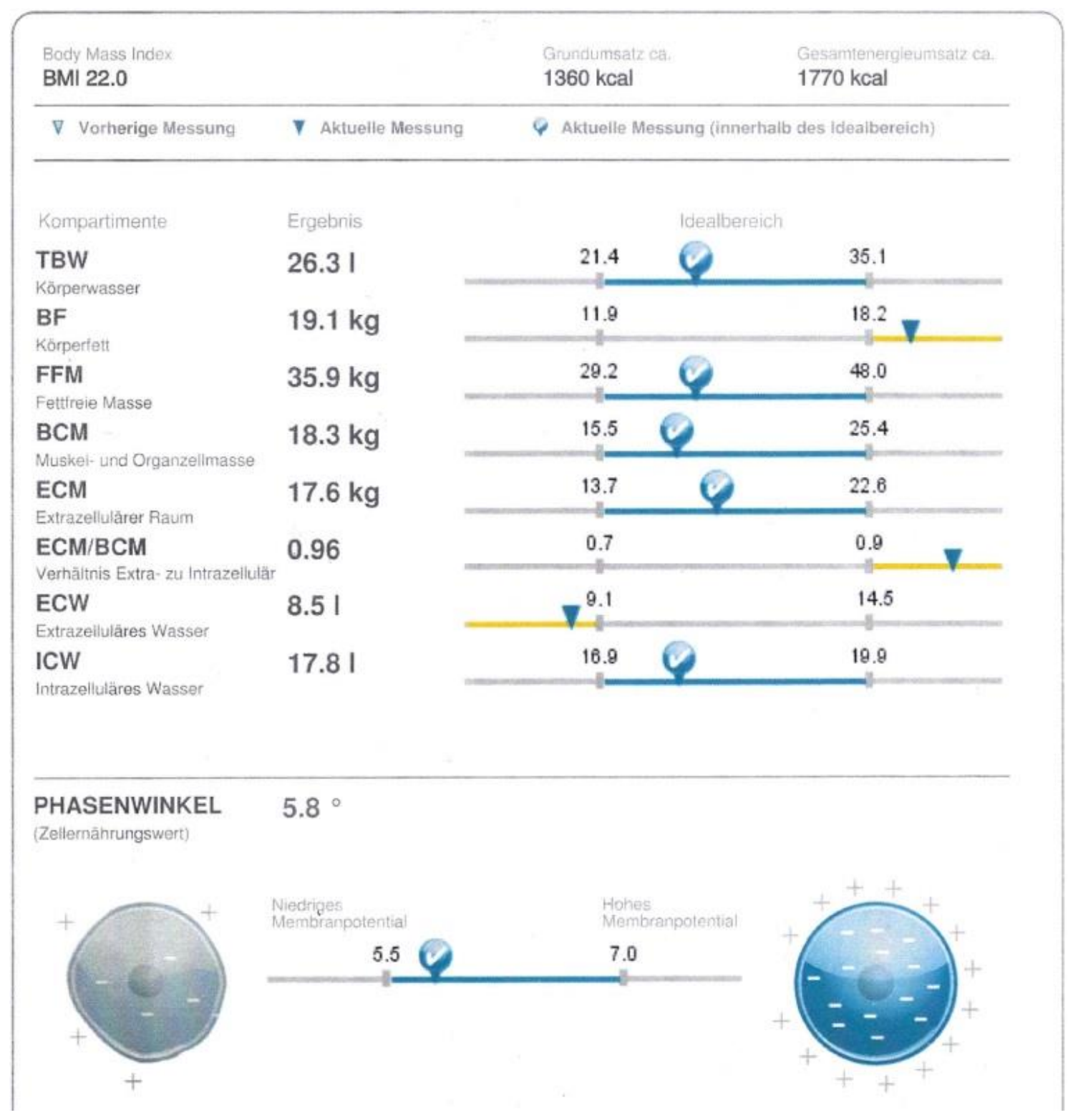

Abbildung 40: Messergebnisse der restlichen Kompartimente 


\subsubsection{Körperfettwaage}

Die Messung der Körperzusammensetzung mittels Körperanalysewaage erfolgte im Anschluss an die BIA-Messung. Dafür wurde das Modell Body Analysis Scale der Firma Rituals... verwendet (siehe Abbildung 41).

Genau wie bei der BIA wäre auch hier das Tragen eines Herzschrittmachers/Defibrillators ein Ausschlusskriterium für die Durchführung der Messung mit der Körperfettwaage gewesen.

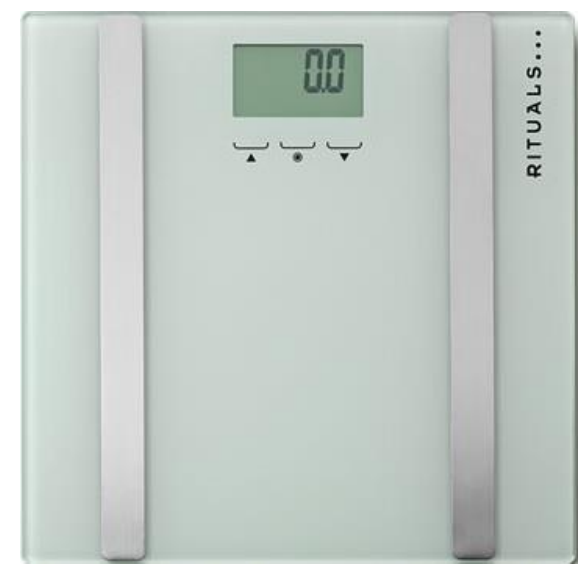

Abbildung 41: In der Studie verwendete Körperfettwaage

Alle weiteren Voraussetzungen für die Durchführung der Messung (Schuhe und Kleidung bis auf die Unterwäsche ausziehen) waren bereits erfüllt, da sich die Patientinnen Barfuß auf die Fettwaage stellen mussten.

Nach dem Einschalten und vor dem Betreten der silbernen Messstellen (integrierte

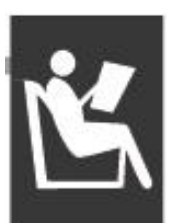
Elektroden) der Waage, musste zunächst ein Speicherplatz für die Messung gewählt, anschließend das Geschlecht, dann das Aktivitätsniveau (siehe Abbildung 42) ausgewählt, im nächsten Schritt die Körpergröße und abschließend das Alter eingegeben werden.

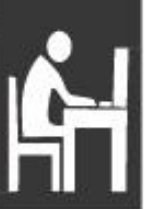

Bei der Messung musste sichergestellt sein, dass die Waage auf festem, ebenem Untergrund stand, dass die Patientinnen beide Beine gleichstark belasteten und v. a., dass die Füße absolut trocken waren.

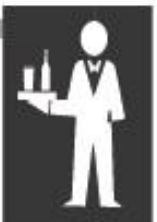

Zunächst wurde nach Betreten der Waage das Körpergewicht und nach wenigen Sekunden weiterer Berechnung dann die Ergebnisse für Körperfett

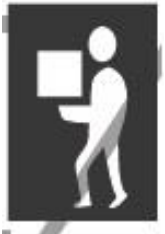
(BF), Körperwasser (BW), Muskelmasse (BM), Knochenmasse (BONE) und der Tageskalorienbedarf (kcal) angezeigt (siehe Abbildung 43).

Lagen die gemessenen Werte außerhalb der in Abbildung 44 angegeben

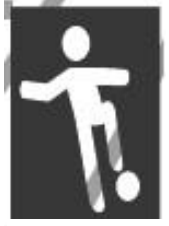
Messbereiche, wurde die Fehlermeldung ERROR angezeigt. Wie im Ergebnisteil sichtbar, war dies bei einigen Patientinnen der Fall und es sind ent- 
sprechend keine Werte vorhanden, die statistisch und vergleichend ausgewertet werden konnten.

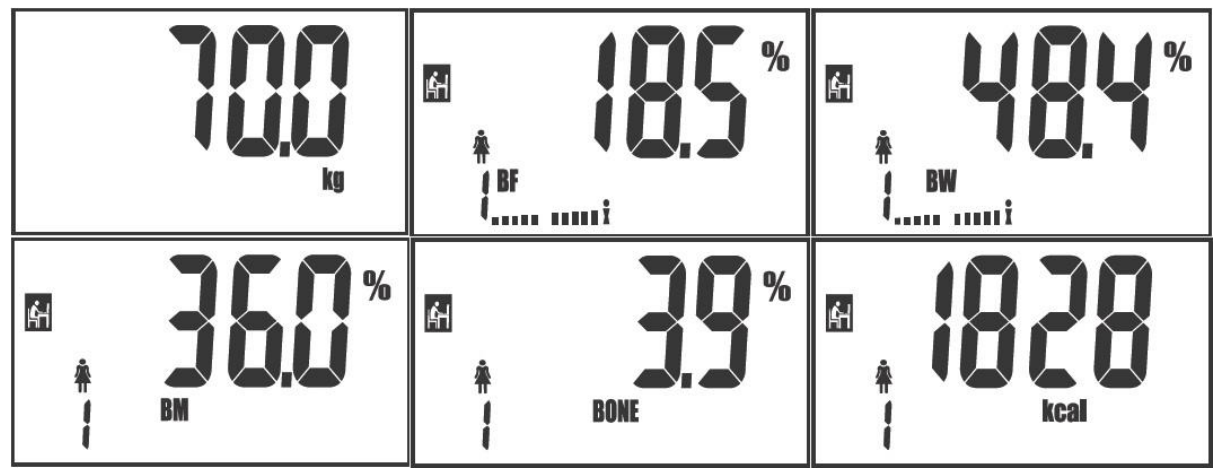

Abbildung 43: Berechnete und angezeigte Messergebnisse

\begin{tabular}{|c|c|c|c|c|c|c|}
\hline & $\begin{array}{l}\text { Körper- } \\
\text { gewicht }\end{array}$ & Fett \% & Wasser $\%$ & Muskeln \% & Knochen \% & $\begin{array}{c}\text { Kalorien- } \\
\text { bedarf }\end{array}$ \\
\hline $\begin{array}{l}\text { Mess- } \\
\text { bereich }\end{array}$ & $\begin{array}{c}150 \mathrm{~kg} \\
\sim 330 \mathrm{lb}\end{array}$ & $\begin{array}{c}4.0 \% \\
\sim 60.0 \%\end{array}$ & $\begin{array}{c}27.5 \% \\
\sim 66.0 \%\end{array}$ & $\begin{array}{c}20.0 \% \\
\sim 56.0 \%\end{array}$ & $\begin{array}{c}2.0 \% \\
\sim 20.0 \%\end{array}$ & \\
\hline
\end{tabular}

Abbildung 44: Minimaler- bzw. maximaler Messbereich

Die Interpretation der Tendenzbalken sowie die Referenzwerte für Körperfett- und Wasseranteil sind in Abbildung 77 dargestellt (siehe Anhang).

\subsubsection{Nahinfrarot-Spektroskopie}

Die letzte der vier Messungen in der Reihe zur Bestimmung der Körperzusammensetzung wurde mit dem FUTREX 6100A/ZL (Hersteller FUTREX Inc.) durchgeführt (siehe Abbildung 45). Dieses Gerät ist sowohl für die Messung bei Erwachsenen ab 18 Jahren als auch für Kinder ab 5 Jahren kalibriert. Die gemessenen Werte ließen sich zum einen mittels integriertem Drucker direkt nach der Messung ausdrucken und zum anderen zusätzlich noch zur genaueren Differenzierung mittels Auswertesoftware (Body Composition Analysis 2013 - 3.5.158)

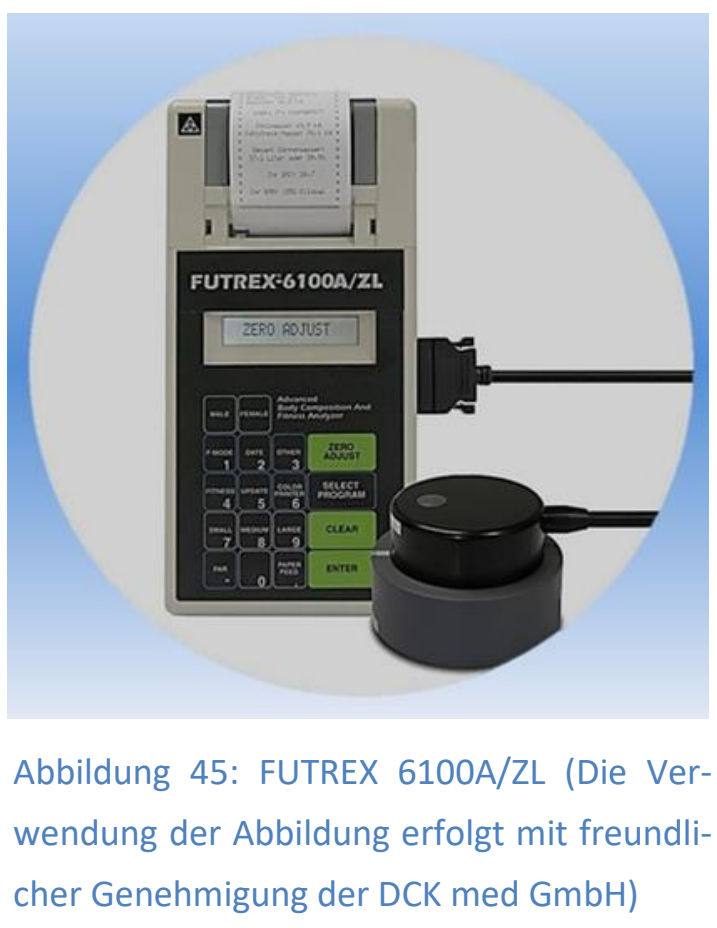

cher Genehmigung der DCK med GmbH) 
am PC aufschlüsseln. Vor jeder neuen Messung musste eine Kalibrierung/Nullanpassung des Lichtstabs (= Messsonde) im optischen Standard (= Kalibrationsstation) durchgeführt werden und anschließend nacheinander Geschlecht, Geburtsdatum, Größe (in $\mathrm{cm}$ ) und Gewicht (in kg) eingegeben werden. Um bei der nächsten Messung Zeit zu sparen, hätten diese Daten unter einer frei wählbaren ID-Nummer gespeichert werden können.

Falls gewünscht, bietet das FUTREX 6100A/ZL zusätzlich noch die Möglichkeit, eine Fitnessanalyse durchzuführen. Hierfür ist zusätzlich die Eingabe von Knochenbau (SMALL-MEDIUM-LARGE), von Grad der körperlichen Betätigung (1-2-3) und von Grad der sportlichen Betätigung (Häufigkeit 1 - 5, Intensität 1 - 5, Dauer 1 - 4) erforderlich.

Um den genauen Messpunkt auf dem Biceps des dominanten Armes zu ermitteln, wurde der beiliegende Biceps Locator verwendet. Der Locator wurde so von Beginn der Achselhöhle am ausgestreckten Arm entlang angelegt, dass das Ende TO ELBOW in Richtung Innenseite des Ellbogens zeigte (siehe Abbildung 46). Die so gemessene Länge zwischen dem Beginn der Achselhöhle und der Innenseite des Ellbogens konnte auf der großen Skala abgelesen werden und die entsprechende Zahl auf der kleinen Skala stellte dann die Markierung für den genauen Messpunkt auf dem Biceps dar.

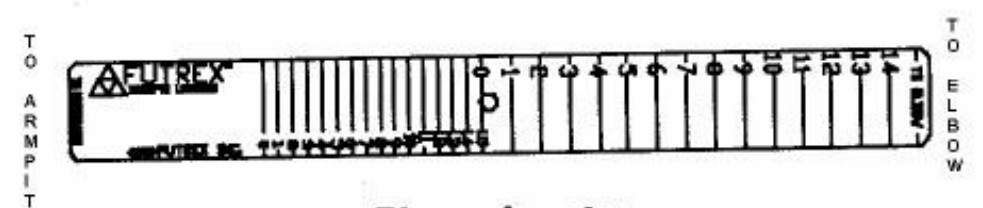

\section{Biceps Locator}

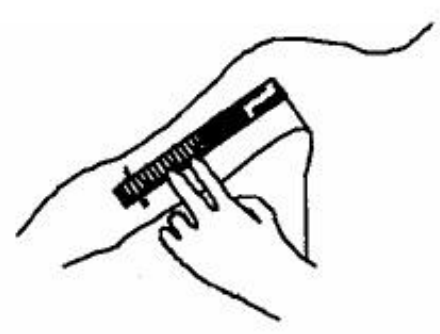

Abbildung 46: Bestimmung des Messpunktes am Biceps

Während der Messung musste die Muskulatur des Oberarmes und v. a. die des Biceps entspannt sein, was am besten durch Auflegen des Ellbogens auf einen Tisch erreicht wurde. Außerdem wurde darauf geachtet, dass der Oberarm gegen den Unterarm annähernd im rechten Winkel stand und dass die Handfläche die ganze Zeit nach oben zeigte. Damit kein Licht von außen das Ergebnis der Messung verfälschen konnte, wurde der Lichtstab im Lichtschutz mit beiden Händen auf den Messpunkt aufgedrückt. Generell sollte die Messung mittels Nahinfrarot-Gerät nicht bei starkem Sonnenlicht 
oder in hell beleuchteten Räumen durchgeführt werden, um fehlerhafte Messwerte durch Streulicht zu vermeiden. Um im Gegensatz dazu die möglicherweise vollständige Absorption des NIR-Lichtes und die damit verbundene Fehlmessung zu umgehen, sollte die Messung nicht auf extrem dunklen Tattoos durchgeführt werden (Jones und Spears 2014).

Die eigentliche Messung dauerte anschließend nur wenige Sekunden. Nach der Aktivierung der Messung durch einen Knopf auf dem Lichtstab erfolgte die erste Messung. Nach kurzer Zeit musste der Lichtstab kurz seitlich vom Biceps abgehoben, auf demselben Messpunkt wieder aufgesetzt und die Messung ein zweites Mal ausgelöst werden.

Erst nachdem der Thermodrucker automatisch aktiviert worden war und mit dem Drucken der Ergebnisse begann, konnte der Lichtstab weg vom Arm zurück in die Kalibrationsstation gelegt werden. Das Ergebnis dieses Drucks ist beispielhaft in Abbildung 47 dargestellt. Neben den vor der Messung eingegeben Daten waren nun das Körperfett (\%) und entsprechend die Fettmasse (kg), die fettfreie Masse (kg), das GesamtKörperwasser (I und \%) und schließlich der errechnete BMI abzulesen.

Nach der Übernahme von Geschlecht, Körpergröße, Gewicht und Körperfettanteil (in \%) vom ausgedruckten Ergebnis-Zettel in das Auswerteprogramm am PC konnten die in Abbildung 48 dargestellten weiteren Differenzierungen der einzelnen Kompartimente vorgenommen werden.

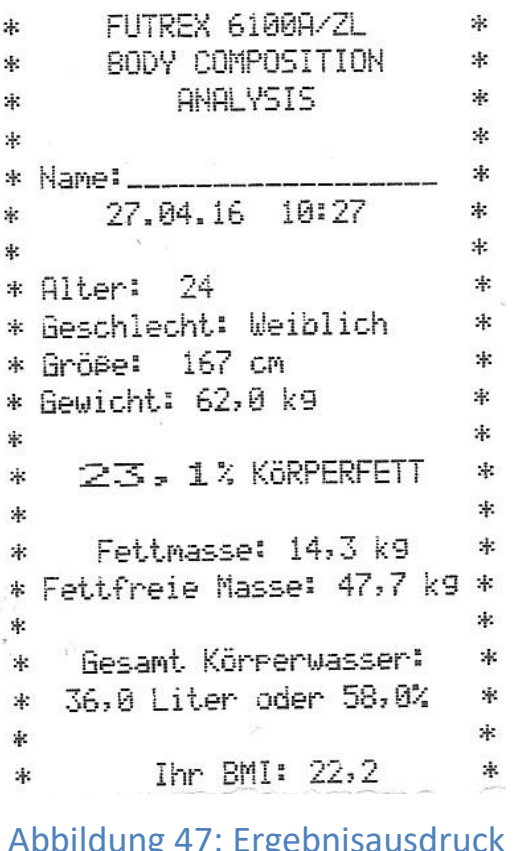

Abbildung 47: Ergebnisausdruck

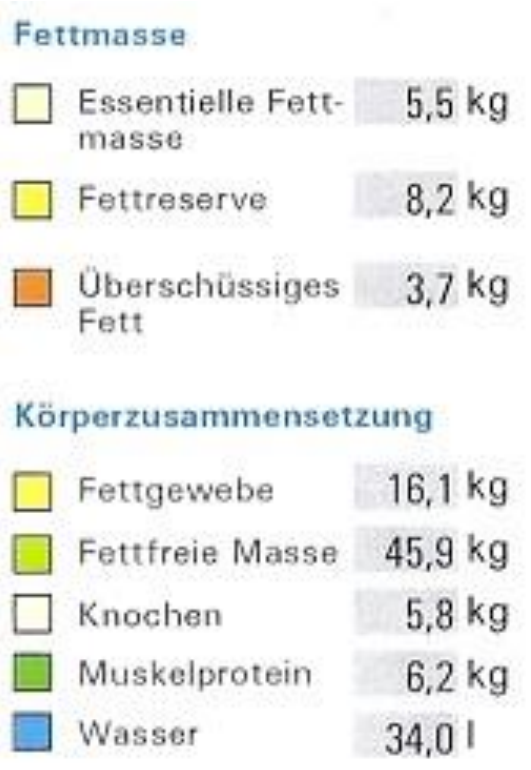

Abbildung 48: Weitere Differenzierung der Kompartimente mittels FUTREX-Auswertesoftware 


\subsubsection{Spiroergometrie}

Die Spiroergometrie als Untersuchung zur Bestimmung der körperlichen Leistungsfähigkeit wurde immer Montag- und Freitagvormittag zwischen 10 und 12 Uhr durchgeführt.

Vor der eigentlichen Spiroergometrie-Messung wurde auch eine Ruheumsatzbestimmung (RER in Ruhe) durchgeführt. Als Orientierung für die Standarduntersuchungsbedingungen diente ein Artikel von Compher et al. (2006), in dem ein Überblick über die verschiedenen Voraussetzungen für eine möglichst exakte Ruheumsatzbestimmung gegeben wird. Die Autoren werteten darin 18 indirekte Kalorimetrie-Messmethoden aus, gruppierten sie anhand von 10 Fragen ein und überprüften ihre Anwendbarkeit auf verschiedene Patientenkollektive.

9 von diesen 10 Punkten wurden so gut wie möglich bei den KOLIBRI-Patientinnen umgesetzt:

1. Mögliche Schwankungen der Messwerte durch unterschiedliche Tageszeiten wurden durch den o. g. festen Zeitraum minimiert.

2. Letzte kleine Mahlzeit $4 \mathrm{~h}$ vor Untersuchung (oftmals gewährleistet, da Frühstück zwischen 7 - 8 Uhr) und mindestens $2 \mathrm{~h}$ vorher kein Alkoholgenuss; für Art und Menge anderer Getränke gab es hier keine Einschränkungen.

3. $2 \mathrm{~h}$ vor Untersuchung kein Nikotingenuss mehr.

4. Am Morgen (oder mindestens $2 \mathrm{~h}$ vorher) keine leichte körperliche Tätigkeit mehr, letzte erschöpfende sportliche Betätigung vor mehr als $14 \mathrm{~h}$.

5. Mindestens $10-20$ Minuten körperliche Ruhe vor Beginn, wobei normale, alltägliche Betätigungen aufgrund des nur minimalen Einflusses auf die Messwerte gestattet waren.

6. Bequeme Positionierung (hier im Liegen), Wiederholungsmessungen in derselben Position und keine beengende Bekleidung.

7. Umgebungstemperatur zwischen $20-25^{\circ} \mathrm{C}$.

8. Sehr eng sitzende, gut passende Gesichtsmaske.

9. Wenn möglich Ruheumsatzbestimmung im steady state; (hier: Streichen der Messwerte der ersten 5 Minuten und Bilden des Mittelwertes aus den Messwerten der zweiten 5 Minuten) 
In der Vergangenheit wurden traditionell Stufentests verwendet. Die Messtechnik erlaubte es nämlich nicht, die erhobenen Parameter zu einem eng definierten Zeitpunkt zu erfassen, da hierfür ein gewisser Zeitraum im steady state einer Belastungsstufe benötigt wurde. Durch die Entwicklung der Breath-by-Breath-Analyse ist die Dauer der Messwerterfassung nicht mehr relevant, sodass sich zunehmend Rampenprotokolle durchgesetzt haben. Damit werden höhere VO2max-Werte erreicht als beim Stufenprotokoll und die VT2 kann exakter ermittelt werden (Kroidl et al. 2010).

Deshalb wurde auch hier als Belastungsform ein Rampenprotokoll auf einem Fahrradergometer gewählt (Gerät: ergometrics 900 von ergoline; Auswertesoftware: SDS 104 von Ganshorn).

Der Ablauf der Untersuchung war wie folgt:

1.) Eingabe der Patientendaten (Name, Geschlecht, Geburtsdatum, Größe, Gewicht).

2.) Anschließen (und kontinuierliches Ableiten) eines 12 Kanal-EKG's.

3.) Aufziehen der Gesichtsmaske (Ergo Maske von Ganshorn), 10-minütiges Liegen auf einer Liege direkt neben dem Ergometer, dabei einmalig manuelles Messen des Blutdrucks (RR) und Ruheumsatzbestimmung (RER in Ruhe).

4.) Aufsteigen auf das Fahrradergometer und unmittelbarer Beginn der Belastung mittels Rampenprotokoll, erneutes manuelles Messen des RR.

5.) Kurz vor Ende der Belastung wieder RR-Messung, dann 5-minütige Erholungsphase auf dem Fahrradergometer, nach 3 Minuten Messung des Blutlaktatwertes am Ohrläppchen (Lactat photometer von diaglobal).

6.) Kurz vor dem Absteigen ein letztes Mal messen des RR.

Als Abbruchkriterien wurden die folgenden nach Kroidl et al. (2010) definiert:

- Klinische Symptomatik:

- Brustschmerzen

- Plötzliche Blässe, Verwirrung, Schwindel

- Dyspnoe/Ateminsuffizienz

- EKG:

- Signifikante ST-Streckenveränderungen

- Komplexe Rhythmusstörungen 
- $\quad$ AV-Block $11^{\circ} / I^{\circ}$

- Schenkelblock (neu)

- Blutdruck:

- $\quad$ Anstieg $>250 / 120 \mathrm{mmHg}$

- Abfall > 20 mmHg vom höchsten Wert

Die weitgehende Ausbelastung ist die entscheidende Voraussetzung für eine valide Beurteilung der Leistungsfähigkeit. Die exakte Bestimmung des Zeitpunktes der maximalen Ausbelastung ist allerdings schwierig.

Als Indizien für eine Ausbelastung nennen Kroidl et al. (2010) beispielsweise:

- Plateaubildung der Herzfrequenz (maximale Herzfrequenz: 220 - Lebensjahre bei Gesunden bzw. 200 - Lebensjahre bei Erkrankten)

- Plateaubildung in der Sauerstoffaufnahme (Levelling-off)

- $\operatorname{RER} \geq 1$

- Atemfrequenz $>50 / \mathrm{min}$

- Laktatwerte $>$ 8-10 $\mathrm{mmo} / \mathrm{I}$

In unserem Falle war RER $\geq 1$ das entscheidende Kriterium. Einige der Patientinnen erreichten diesen Wert nicht und mussten die Untersuchung aufgrund muskulärer Erschöpfung vorher abbrechen.

In Abbildung 49 und 50 sind Ausschnitte eines Ergebnisausdruckes beispielhaft abgebildet. 


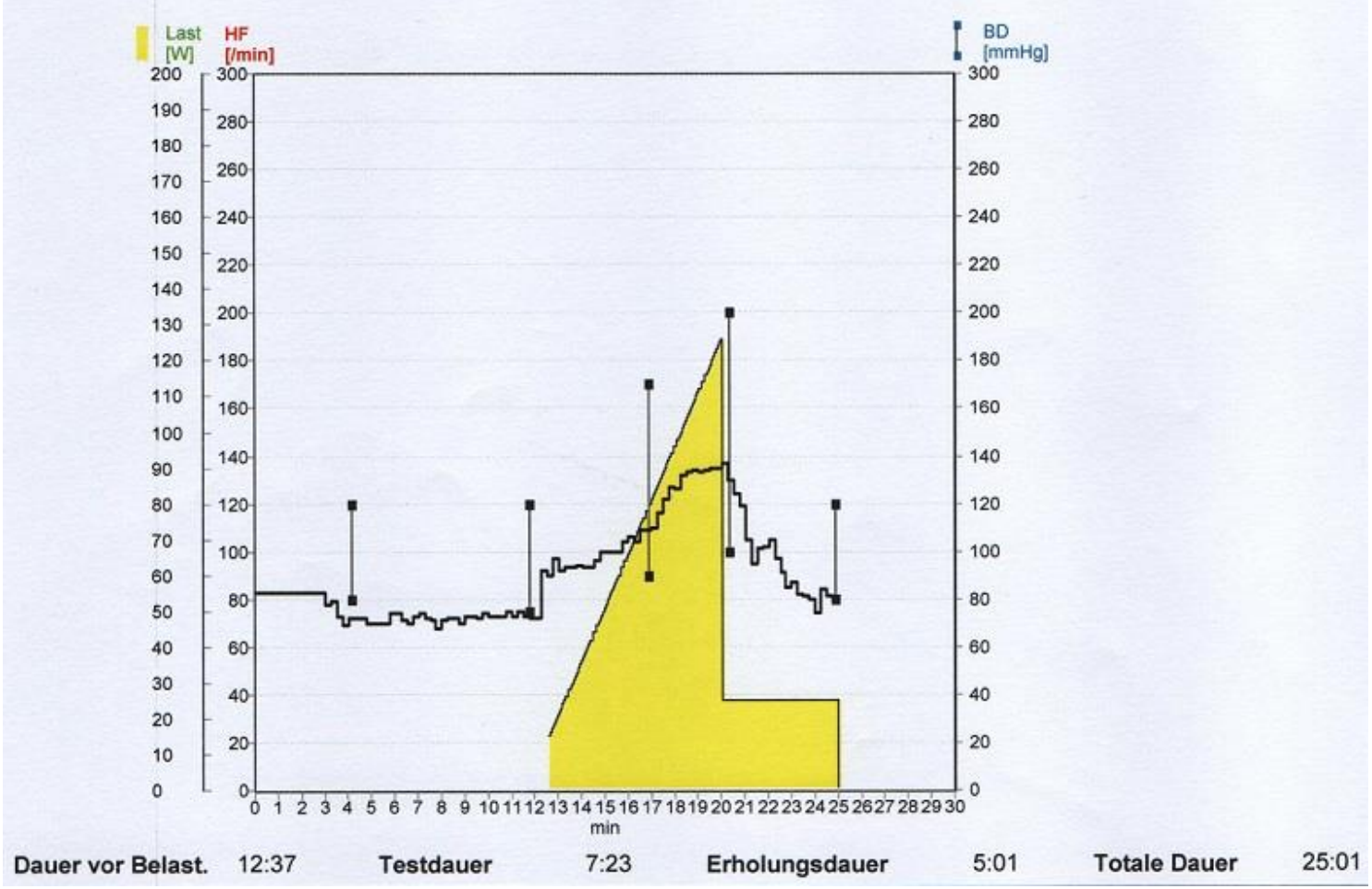

Abbildung 49: Beispielhaftes Schaubild einer kompletten Belastung

\begin{tabular}{|c|c|c|c|c|c|c|c|}
\hline & Soll & Ruhe & AT & Max.Last & Max/Soll & AT/Soll & Erhol. \\
\hline Zeit ................... h:mm:ss & - & $0: 11: 40$ & $0: 17: 50$ & $0: 19: 20$ & - & - & $0: 21: 20$ \\
\hline Last ............................... & 125 & - & 103 & 126 & $101 \%$ & $82 \%$ & 25 \\
\hline VO2 …..................... $1 / \min$ & 1,45 & 0,27 & 1,30 & 1,46 & $101 \%$ & $90 \%$ & 0,58 \\
\hline $\mathrm{VO} 2 / \mathrm{kg} \ldots \ldots . \mathrm{ml} /\left(\mathrm{min}^{*} \mathrm{~kg}\right)$ & 18,34 & 3,47 & 16,46 & 18,48 & $101 \%$ & $90 \%$ & 7,39 \\
\hline VCO2 & 1,59 & 0,23 & 1,30 & 1,56 & $98 \%$ & $81 \%$ & 0,75 \\
\hline RER & - & 0,83 & 1,00 & 1,07 & - & - & 1,28 \\
\hline \multicolumn{8}{|l|}{ Zirkulation } \\
\hline 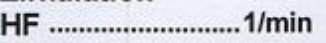 & 145 & 91 & 145 & 135 & $93 \%$ & $100 \%$ & 96 \\
\hline O2-Puls …..........ml/beat & 11,03 & 3,01 & 8,97 & 10,81 & $98 \%$ & $81 \%$ & 6,08 \\
\hline BDsys ............... mmHg & - & 120 & 170 & 170 & - & - & 200 \\
\hline BDdia …............ mmHg & - & 75 & 90 & 90 & - & - & 100 \\
\hline \multicolumn{8}{|l|}{ Ventilation } \\
\hline VE & 50,55 & 9,07 & 37,66 & 44,81 & $89 \%$ & $74 \%$ & 21,34 \\
\hline VT & 1,58 & 0,31 & 1,29 & 1,54 & $98 \%$ & $82 \%$ & 0,50 \\
\hline $\mathrm{AF}$ & 30 & 29 & 29 & 29 & $97 \%$ & $97 \%$ & 42 \\
\hline BR ...................................... & - & 89 & 56 & 47 & - & - & 75 \\
\hline VDNT & - & - & - & - & - & - & - \\
\hline
\end{tabular}

Abbildung 50: Entsprechende Messergebnisse der Belastung (passend zu Abbildung 49) 


\section{Ergebnisse}

\subsection{Statistik}

Für die statistische Auswertung der Messergebnisse wurden Varianzanalysen (ANOVA's; engl. für Analyses of Variance) mit Messwiederholungen durchgeführt. Mit diesem datenanalytischen Verfahren konnte jeweils zu den Zeitpunkten t0 und t1 und im zeitlichen Verlauf (t0 - t1) getestet werden, ob sich die Mittelwerte der Messergebnisse im Gruppenvergleich voneinander unterschieden. Da die Varianzanalysen jedoch keine Auskunft darüber geben konnten, welche Mittelwerte genau sich jeweils signifikant von den anderen unterschieden, mussten anschließend Post-hoc-Tests berechnet werden. Bei dieser Art von Test wird durch paarweisen Vergleich der Gruppen geprüft, welche Mittelwertunterschiede zur entsprechend signifikanten Varianzanalyse geführt haben. Um das Problem der zunehmenden Wahrscheinlichkeit für die irrtümliche Ablehnung der Nullhypothese (alpha-Fehler) bei zunehmender Anzahl der Vergleiche zu beheben, wurde im Rahmen der o. g. Post-hoc-Tests automatisch die sog. BonferroniKorrektur angewendet (Division von alpha durch die Anzahl der Paarvergleiche) (Hochschule Luzern, Universität Zürich 2016).

Die nachfolgende grafische Darstellung aller Ergebnisse erfolgt hauptsächlich mit Hilfe von Mittelwert-Plots.

\subsection{Körperzusammensetzung - Zeitpunkt to}

Körpergewicht (siehe Abbildung 51)

Die Betrachtung der Varianzanalyse für das Körpergewicht im Gruppenvergleich (unabhängig von der Messmethode) ergab einen signifikanten Unterschied $(p=0,016)$. Hierbei war der Unterschied zwischen KETO und LOGI signifikant $(p=0,016)$, nicht allerdings zwischen KETO und DGE $(p=0,610)$ und auch nicht zwischen LOGI und DGE $(p=0,515)$.

Das durchschnittliche Gewicht (gemittelt über die Ergebnisse aller Methoden) betrug bei KETO 66,33 kg, bei LOGI 74,11 kg und bei DGE 70,53 kg. 
Beim Methodenvergleich (unabhängig von der Ernährungsform) ergab die Varianzanalyse ebenfalls einen signifikanten Unterschied $(p<0,01)$. Hierbei unterschieden sich die Messergebnisse der DXA jeweils signifikant von denen der anderen drei Messmethoden ( $p$-Wert jeweils $<0,01$ ), während es im Vergleich der anderen Messmethoden untereinander keine signifikanten Unterschiede gab ( $p$-Wert jeweils 1,0 ).

Die Varianzanalyse der Interaktion von "Methode*Gruppe“ ergab keinen signifikanten Unterschied $(p=0,186)$. Bei der detaillierteren Betrachtung der Post-hoc-Tests ließ sich allerdings innerhalb jeder Gruppe ein signifikanter Unterschied zwischen den Ergebnissen der DXA und denen der anderen drei Methoden feststellen ( $p$-Wert jeweils $<0,01$ ).

\section{Mittelwert-Plot - Körpergewicht (t0)}

Effekt von ,,Gruppe": $p=0,016$

Effekt von ,Methode": $p<0,01$

Interaktion von ,,Methode*Gruppe": $p=0,186$

(Vertikale Balken zeigen 0,95 Konfidenzintervalle)

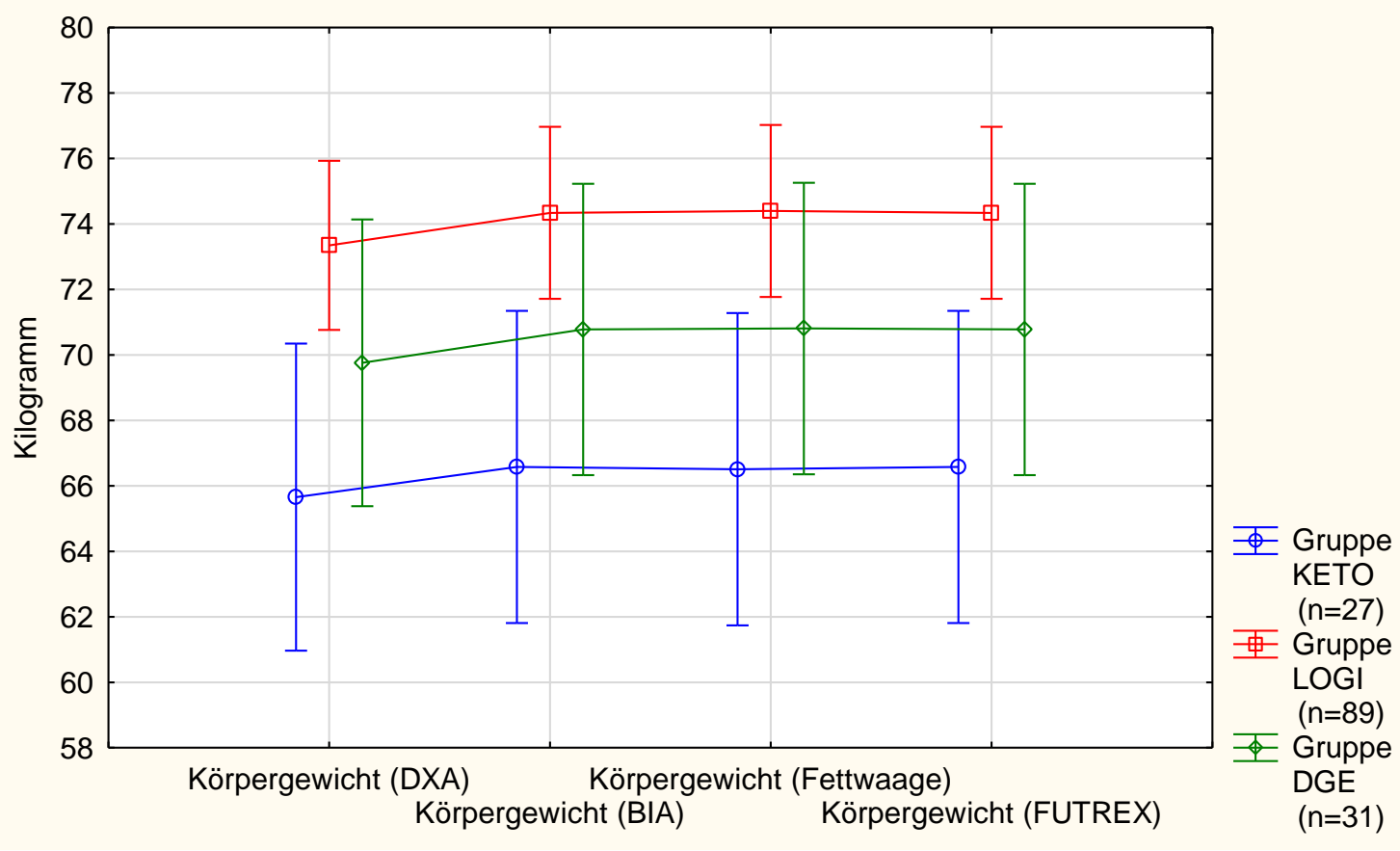

Abbildung 51: Mittelwert-Plot - Körpergewicht (t0)

\section{Fettmasse (siehe Abbildung 52)}

Im Gruppenvergleich (unabhängig von der Messmethode) ergab sich bei der Fettmasse ebenfalls ein signifikanter Unterschied $(p<0,01)$. Auch hier unterschieden sich KETO und LOGI signifikant $(p<0,01)$, nicht aber KETO und DGE $(p=0,092)$, genauso wenig wie LOGI und DGE $(p=1,0)$. 
Die durchschnittlichen Fettmassen (gemittelt über die Ergebnisse aller Methoden) betrugen bei KETO 20,68 kg, bei LOGI 25,52 kg und bei DGE 24,61 kg.

Mit $p<0,01$ ergab sich auch hier ein signifikanter Unterschied im Methodenvergleich (unabhängig von der Ernährungsform). Zusätzlich zu den bereits in der Grafik deutlich sichtbaren Unterschieden zwischen den Messergebnissen der Fettwaage und denen der anderen drei Methoden ( $p$-Wert jeweils $<0,01)$, ergaben sich weitere signifikante Unterschiede, und zwar zwischen DXA und BIA $(p=0,014)$ und zwischen DXA und FUTREX $(p<0,01)$.

Im Gegensatz zum Körpergewicht war bei der Fettmasse die Varianzanalyse der Interaktion von „Methode*Gruppe" signifikant $(p<0,01)$.

Bei den Post-hoc-Tests unterschieden sich in allen drei Gruppen die Messergebnisse der Fettwaage jeweils signifikant von denen der anderen Messmethoden ( $p$-Wert jeweils $<0,01)$. Zusätzlich machte es innerhalb LOGI einen signifikanten Unterschied, ob die Fettmasse mittels DXA oder FUTREX gemessen wurde $(p<0,01)$.

\section{Mittelwert-Plot - Fettmasse (t0)}

Effekt von ,,Gruppe": $p<0,01$

Effekt von ,,Methode": $p<0,01$

Interaktion von ,,Methode*Gruppe": $p<0,01$

(Vertikale Balken zeigen 0,95 Konfidenzintervalle)

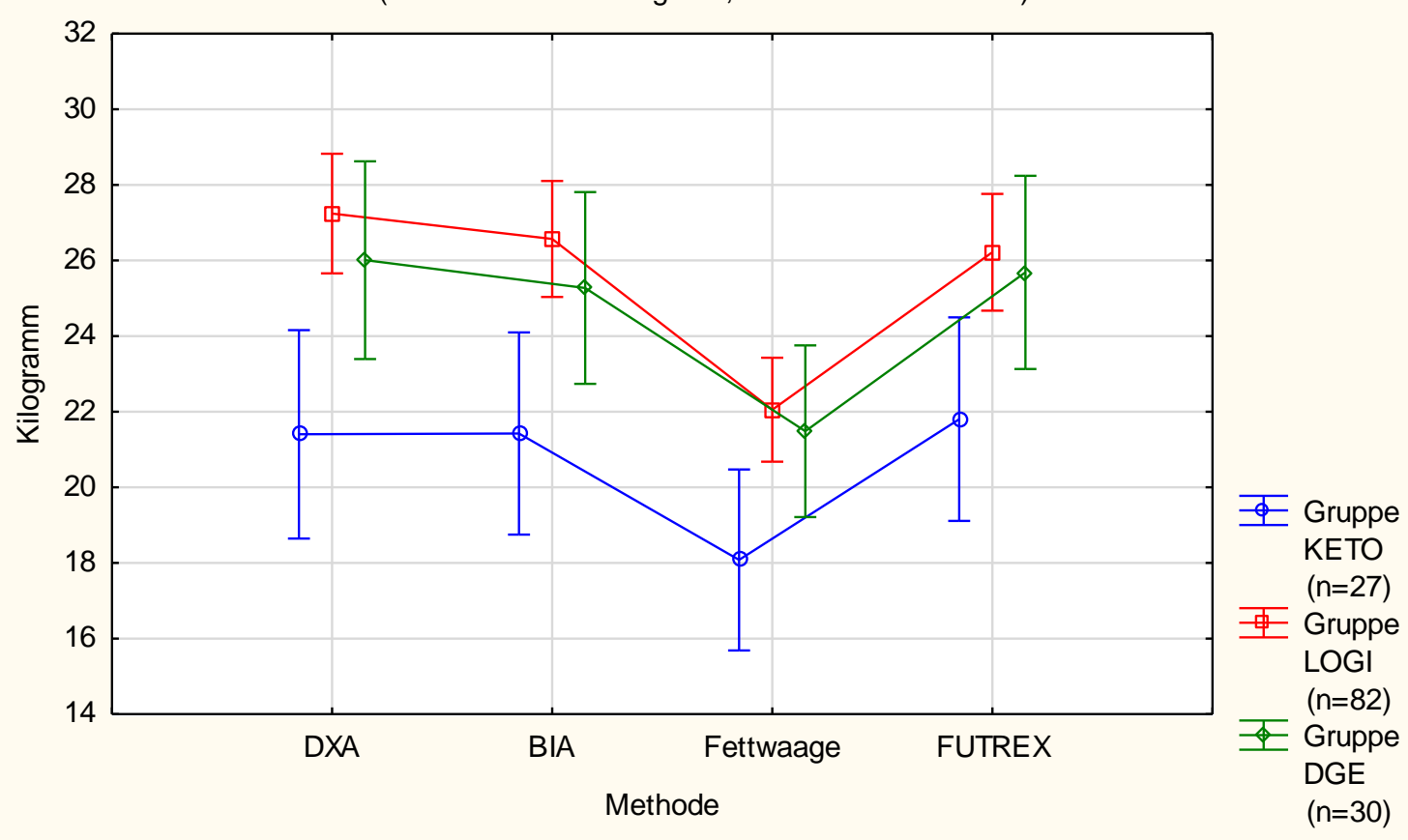




\section{Fettfreie Masse (siehe Abbildung 53)}

Abweichend zu den zwei vorab beschriebenen Parametern Körpergewicht und Fettmasse, ergab sich bei der fettfreien Masse im Gruppenvergleich (unabhängig von der Messmethode) kein signifikanter Unterschied $(p=0,507)$.

Deutlich wird dies bei der Betrachtung der durchschnittlichen fettfreien Massen (gemittelt über die Ergebnisse aller Methoden): KETO 45,65 kg, LOGI 46,13 kg und DGE $45,04 \mathrm{~kg}$.

Im Methodenvergleich (unabhängig von den Gruppen) gab es wiederum einen signifikanten Unterschied $(p<0,01)$; analog zur Fettmasse zwischen den Messergebnissen der Fettwaage und allen anderen Methoden ( $p$-Wert jeweils $<0,01$ ), aber auch zwischen DXA und BIA $(p<0,01)$ und zwischen DXA und FUTREX $(p<0,01)$.

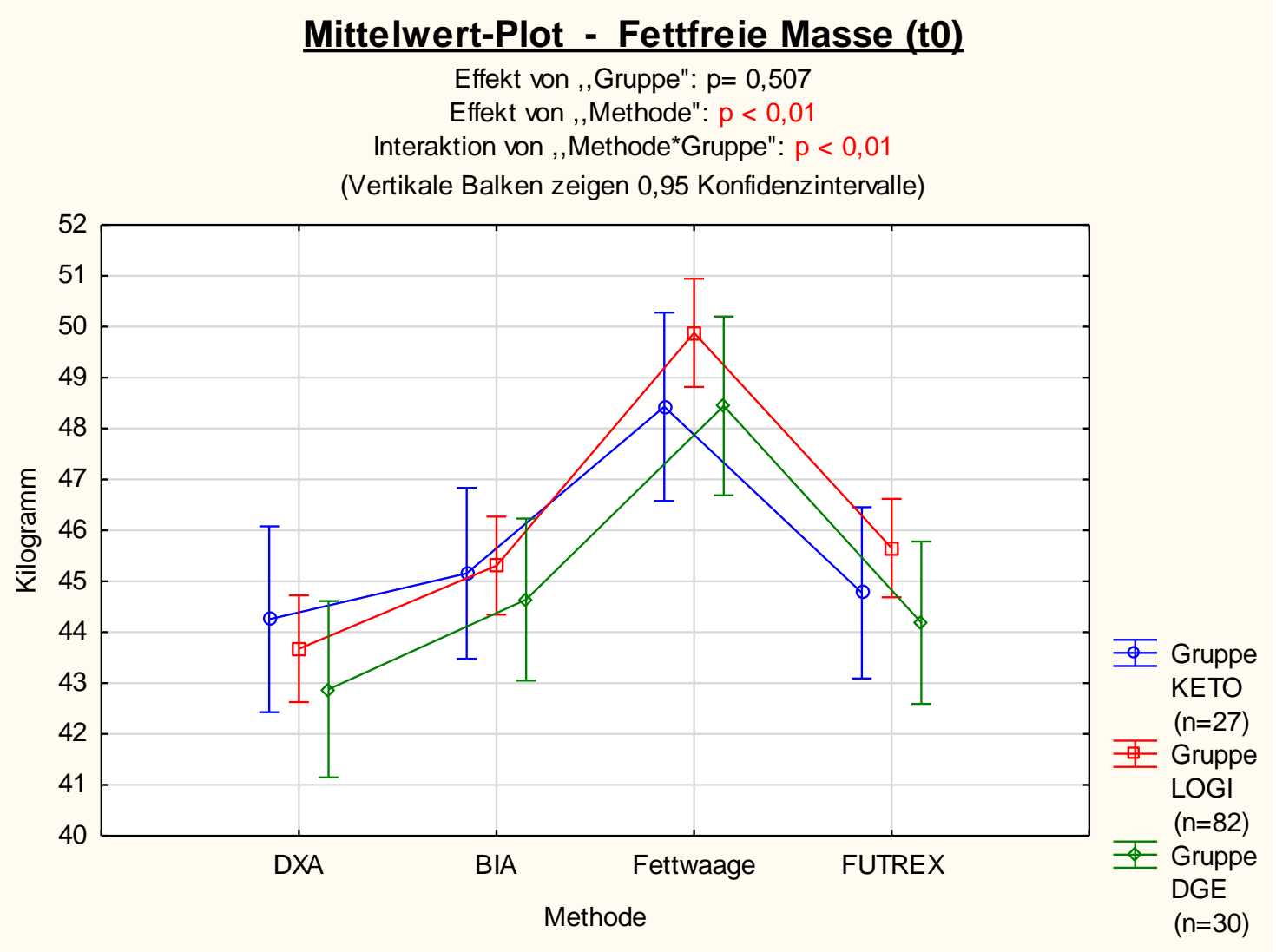

\section{Abbildung 53: Mittelwert-Plot - Fettfreie Masse (t0)}

Bei der Betrachtung, mit welcher Methode innerhalb der drei Gruppen die fettfreie Masse jeweils erhoben wurde, ergaben sich einige signifikante Unterschiede $(p<0,01)$. Analog zur Fettmasse unterschieden sich in allen drei Gruppen die Messergebnisse der 
Fettwaage jeweils signifikant von denen der anderen Methoden ( $p$-Wert jeweils < 0,01). Zusätzlich machte es innerhalb LOGI einen signifikanten Unterschied, ob die fettfreie Masse mittels DXA oder BIA $(p<0,01)$ bzw. mittels DXA oder FUTREX $(p<0,01)$ gemessen wurde. Ebenso verhielt es sich innerhalb DGE zwischen DXA und BIA ( $p<$ $0,01)$.

\subsection{Körperzusammensetzung - Zeitpunkt t1}

\section{Körpergewicht (siehe Abbildung 54)}

Auch zum Zeitpunkt t1 bestand im Gruppenvergleich (unabhängig von der Messmethode) noch ein signifikanter Unterschied $(p=0,038)$. Genau wie bei to war dieser Unterschied zwischen KETO und LOGI signifikant $(p=0,033)$, nicht aber zwischen KETO und DGE $(p=0,330)$ und auch nicht zwischen LOGI und DGE $(p=1,0)$.

Das durchschnittliche Gewicht (gemittelt über alle Messmethoden) betrug bei KETO nun $62,32 \mathrm{~kg}$, bei LOGI 70,51 kg und bei DGE 68,37 kg.

\section{Mittelwert-Plot - Körpergewicht (t1)}

Effekt von ,,Gruppe": $p=0,038$

Effekt von ,Methode": $p<0,01$

Interaktion von ,,Methode*Gruppe": $p=0,999$

(Vertikale Balken zeigen 0,95 Konfidenzintervalle)

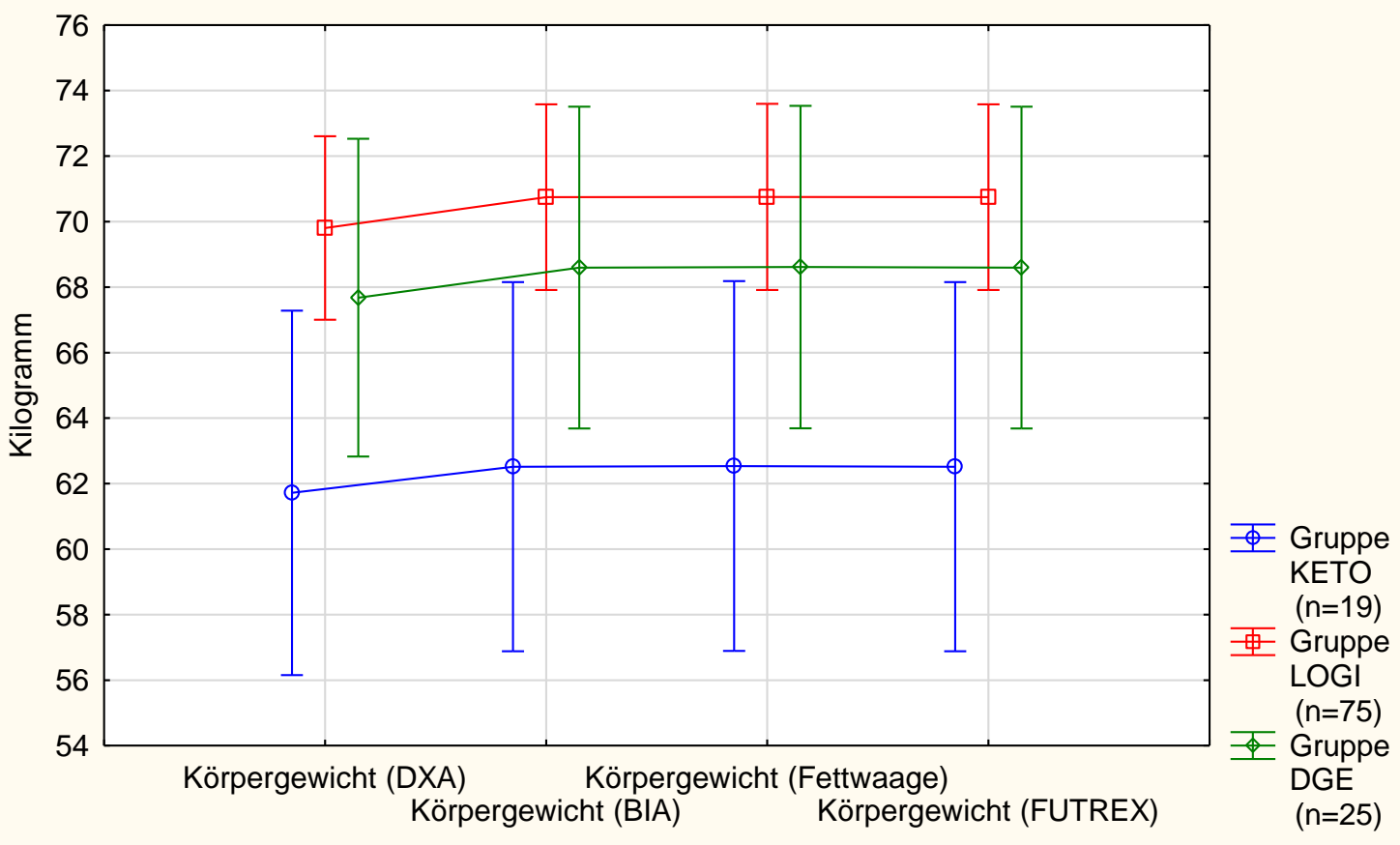


Beim Methodenvergleich (unabhängig von der Ernährungsform) ergab sich keine Änderung der Signifikanz im Vergleich zu t0 $(p<0,01)$. Auch hier unterschieden sich nur die mittels DXA gemessenen Werte für das Körpergewicht signifikant von denen der anderen drei Methoden ( $p$-Wert jeweils $<0,01$ ).

Die Varianzanalyse der Interaktion von „Methode*Gruppe“ ergab auch hier keinen signifikanten Unterschied $(p=0,999)$. Bei der detaillierteren Betrachtung der Post-hocTests fällt im Gegensatz zu to auf, dass nur noch innerhalb LOGI ein signifikanter Unterschied zwischen den Ergebnissen der DXA und denen der anderen drei Methoden zu finden war ( $p$-Wert jeweils $<0,01$ ).

\section{Fettmasse (siehe Abbildung 55)}

Bei der Betrachtung der Fettmasse (unabhängig von der Messmethode) ergab sich ein signifikanter Unterschied im Gruppenvergleich $(p=0,014)$. Anders als bei t0 war der Unterschied sowohl zwischen KETO und LOGI $(p=0,031)$, als auch zwischen KETO und DGE signifikant ( $p=0,018)$; zwischen LOGI und DGE folglicherweise nicht $(p=1,0)$.

Die durchschnittlichen Fettmassen (gemittelt über die Ergebnisse aller Methoden) betrugen bei KETO 18,33 kg, bei LOGI 23,01 kg und bei DGE 24,40 kg.

Im Methodenvergleich (unabhängig von der Ernährungsform) war auch hier der Unterschied signifikant $(p<0,01)$. Im Gegensatz zu t0 waren die Unterschiede zwischen DXA und BIA bzw. zwischen DXA und FUTREX hier bei t1 nicht mehr zu finden, sodass nur noch ein signifikanter Unterschied zwischen den Messergebnissen der Fettwaage und denen der anderen drei Methoden vorlag ( $p$-Wert jeweils $<0,01$ ).

Die Varianzanalyse der Interaktion von „Methode*Gruppe“ erbrachte zwar keinen signifikanten Unterschied $(p=0,439)$, in den Post-hoc-Tests waren allerdings signifikante Unterschiede sowohl innerhalb LOGI als auch innerhalb DGE zwischen den Ergebnissen der Fettwaage und denen der anderen drei Methoden festzustellen ( $p$-Wert jeweils < $0,01)$. 


\section{Mittelwert-Plot - Fettmasse (t1)}

Effekt von ,,Gruppe": $p=0,014$

Effekt von ,,Methode": $p<0,01$

Interaktion von ,,Methode*Gruppe": $p=0,439$

(Vertikale Balken zeigen 0,95 Konfidenzintervalle)

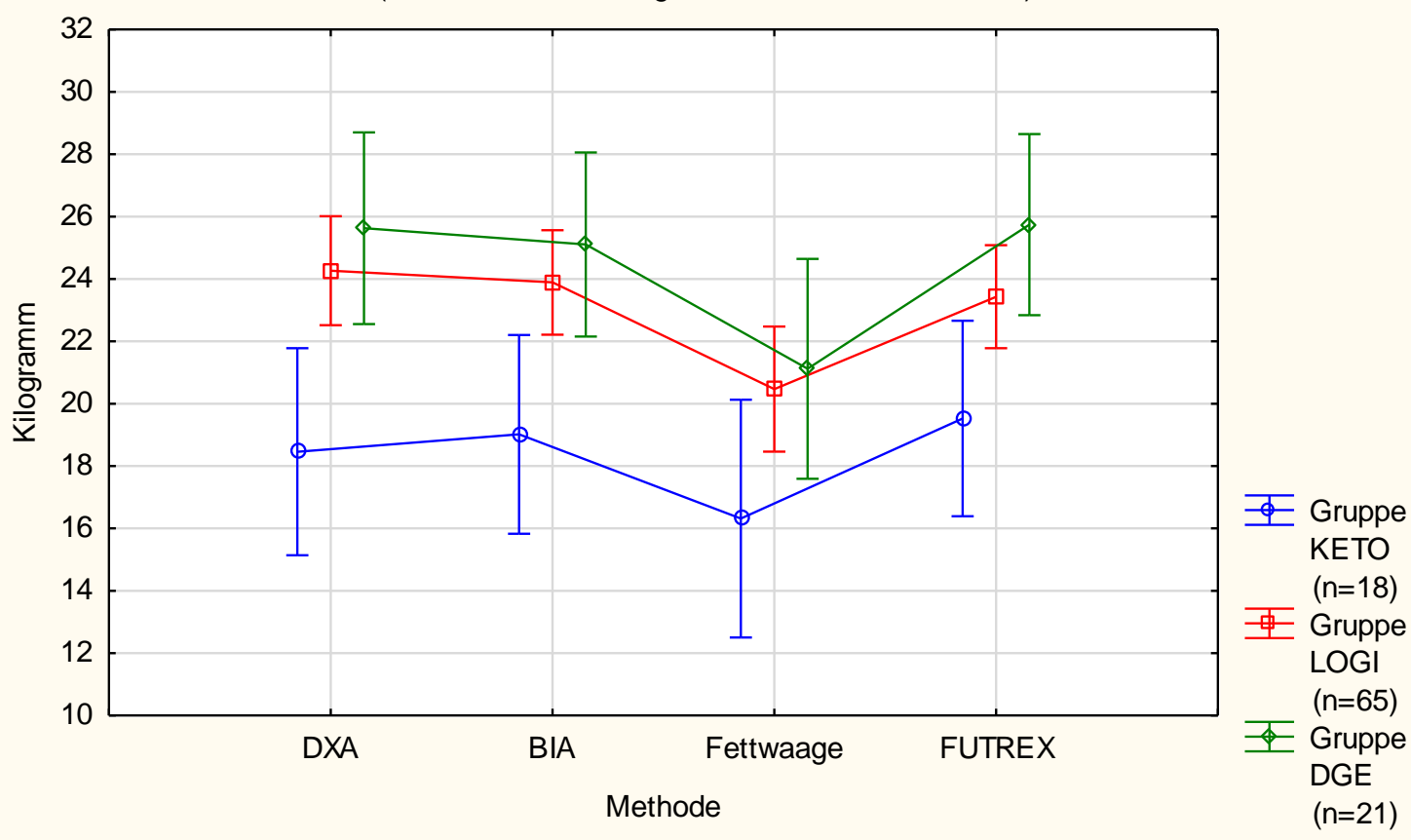

Abbildung 55: Mittelwert-Plot - Fettmasse (t1)

\section{Fettfreie Masse (siehe Abbildung 56)}

Wie der Mittelwert-Plot bereits vermuten lässt, war der p-Wert für den Gruppenvergleich (unabhängig von der Messmethode) deutlich weniger signifikant als bei to ( $p(t)=$ $0,857)$.

Die durchschnittlichen fettfreien Massen (gemittelt über die Ergebnisse aller Messmethoden) unterschieden sich zwischen den Gruppen um nicht einmal $1 \mathrm{~kg}$ (KETO: 44,24 kg; LOGI: 44,61 kg; DGE: 45,19 kg).

Im Methodenvergleich (unabhängig von der Ernährungsform) war der signifikante Unterschied $(p<0,01)$ auch hier nur zwischen den Messergebnissen der Fettwaage und denen der anderen Methoden zu finden. Zwischen der Fettwaage und DXA war der $p$ Wert $<0,01$, zwischen der Fettwaage und BIA betrug er $p=0,029$ und zwischen der Fettwaage und FUTREX $p=0,028$.

Weder bei der Varianzanalyse $(p=0,965)$ noch bei den Post-hoc-Tests waren für die Interaktion von „Methode*Gruppe“ signifikante Unterschiede festzustellen. 


\section{Mittelwert-Plot - Fettfreie Masse (t1)}

Interaktion von ,,Gruppe": $p=0,857$

Interaktion von ,,Methode": $p<0,01$

Interaktion von ,,Methode*Gruppe": $p=0,965$

(Vertikale Balken zeigen 0,95 Konfidenzintervalle)

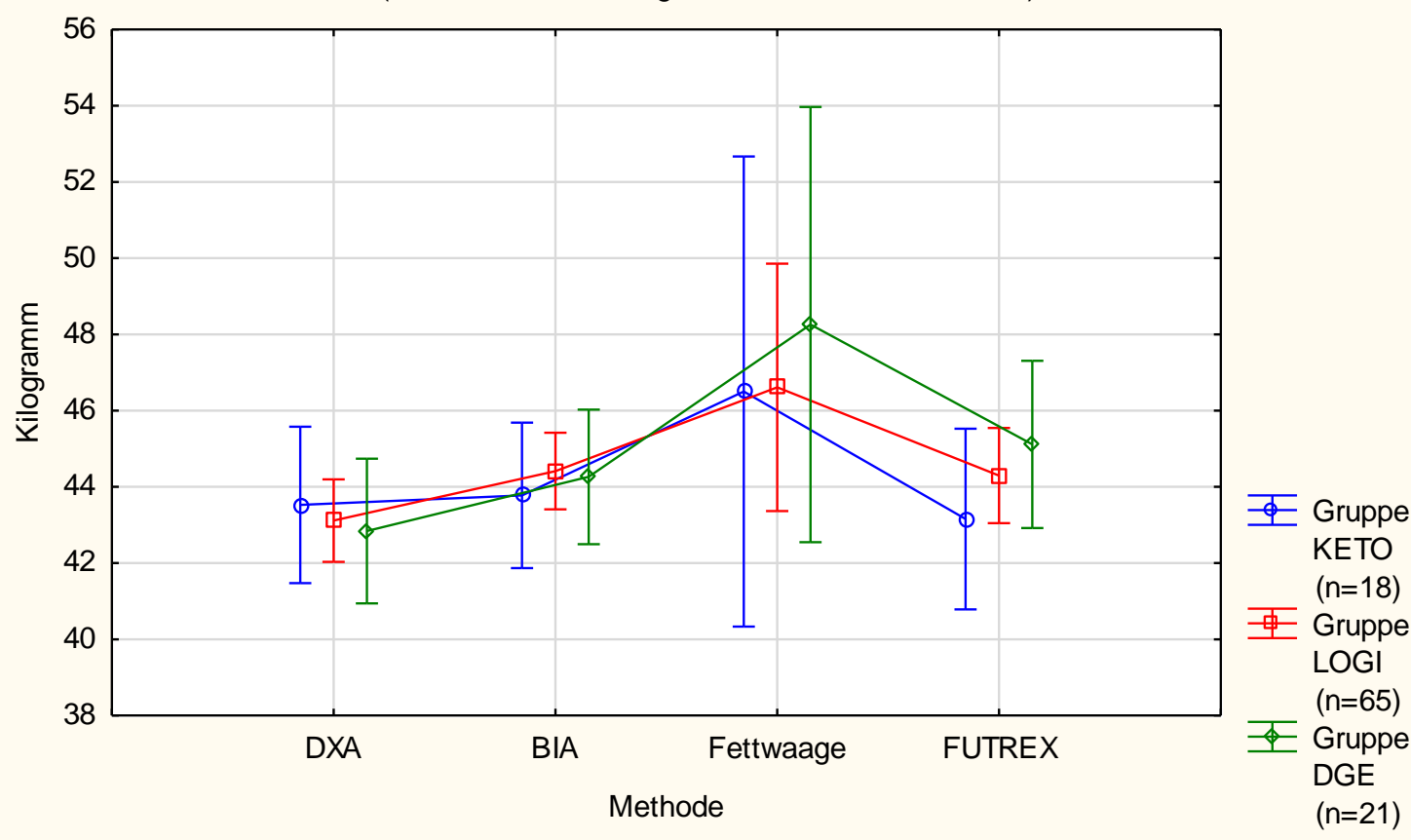

Abbildung 56: Mittelwert-Plot - Fettfreie Masse (t1)

\subsection{Körperzusammensetzung - Zeitlicher Verlauf to - t1}

Vorab wichtig zu erwähnen ist, dass für die Verlaufsbetrachtung nur die Ergebnisse derjenigen Patientinnen verglichen werden konnten, die zu beiden Zeitpunkten (t0 und t1) an den Messungen zur Körperzusammensetzung teilgenommen hatten. Aus diesem Grund unterscheiden sich die hier in den jeweiligen Legenden angegeben Patientinnen-Anzahlen ( $\mathrm{n}$ ) von denen unter 3.2 und 3.3.

\section{Körpergewicht}

Beim hier abgebildeten Mittelwert-Plot (siehe Abbildung 57) sind die durchschnittlichen Körpergewichte (pro Gruppe gemittelt über alle Messmethoden) der drei Gruppen KETO, LOGI und DGE zum Zeitpunkt t0 und t1 dargestellt. Es zeigt sich, dass alle drei Gruppen Gewicht abgenommen hatten. Dabei hatten die Patientinnen innerhalb LOGI mit durchschnittlich 3,58 kg mehr als doppelt so viel Gewicht verloren als diejenigen innerhalb KETO (1,64 kg) bzw. DGE (1,35 kg). 


\section{Mittelwert-Plot - Körpergewicht (t0-t1)}

(gemittelt über alle Messmethoden)

Interaktion von ,Zeitpunkt ${ }^{\star}$ Gruppe": $p<0,01$

(Vertikale Balken zeigen 0,95 Konfidenzintervalle)

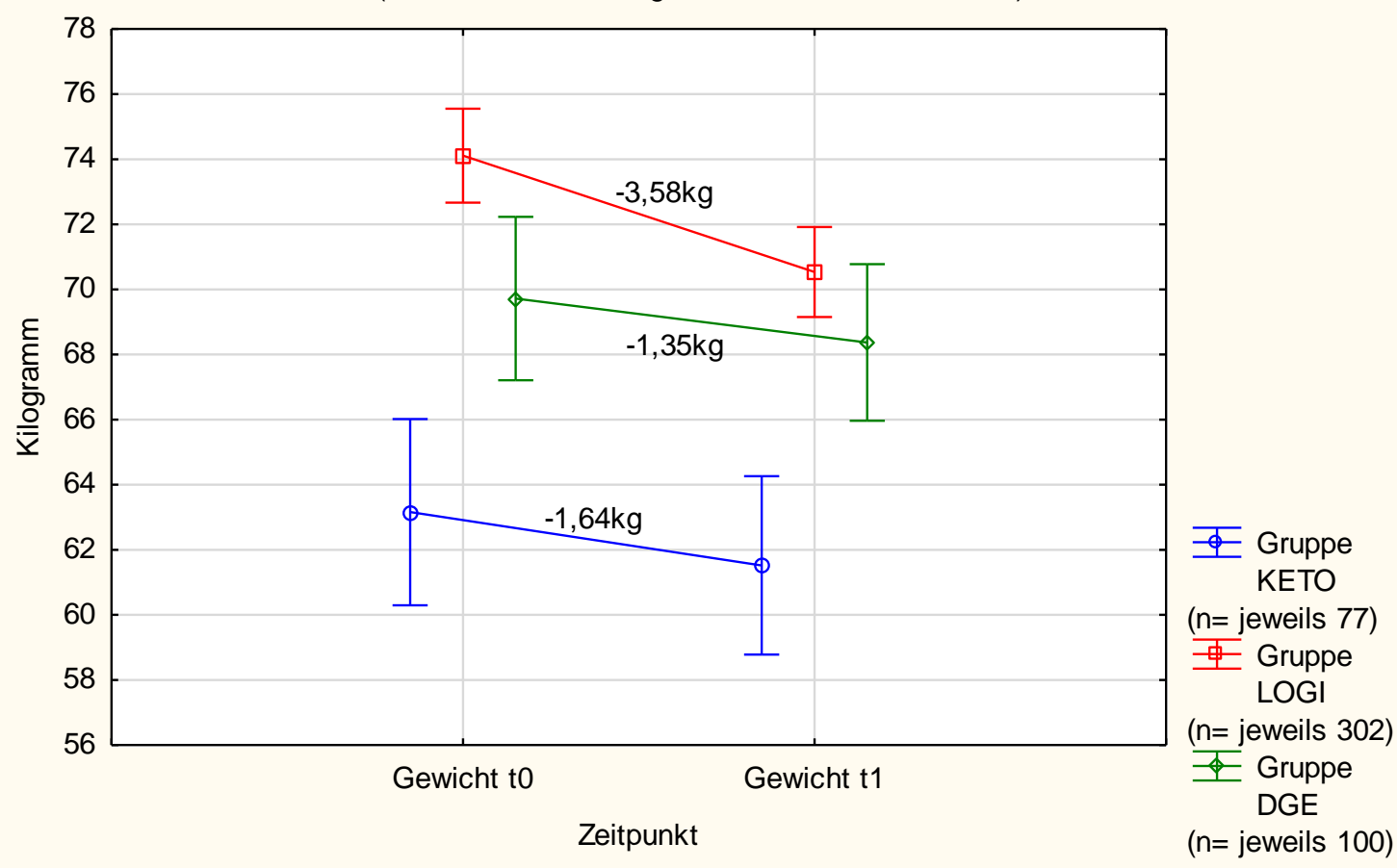

Abbildung 57: Mittelwert-Plot - Körpergewicht (t0-t1)

Im Folgenden wird für jede der drei Gruppen je ein Mittelwert-Plot dargestellt. Darin werden jeweils die Gewichtsverläufe, gemessen mit den vier Messmethoden, betrachtet und miteinander verglichen (siehe Abbildung 58 - 60).

Die Varianzanalyse der Interaktion von „Zeitpunkt*Methode“ ergab bei keiner der drei Gruppen einen signifikanten Unterschied. Bei der detaillierteren Betrachtung der Posthoc-Tests ergaben sich passend zur Tatsache, dass der durchschnittliche Gewichtsverlust innerhalb LOGI im Vergleich zu den anderen beiden Gruppen am größten war, hierfür doch für alle Methoden signifikante $p$-Werte (jeweils < 0,01). D. h., dass sich innerhalb LOGI bei jeder Methode das zum Zeitpunkt t0 gemessene Körpergewicht signifikant vom entsprechend zum Zeitpunkt t1 gemessenen Körpergewicht unterschied. Innerhalb KETO und DGE waren diese Unterschiede nicht zu finden. 


\section{Mittelwert-Plot - Körpergewicht KETO (t0-t1) \\ (Methodenvergleich innerhalb KETO) \\ Interaktion von ,ZZeitpunkt*Methode": $p=\mathbf{0 , 9 8 5}$ \\ (Vertikale Balken zeigen 0,95 Konfidenzintervalle)}

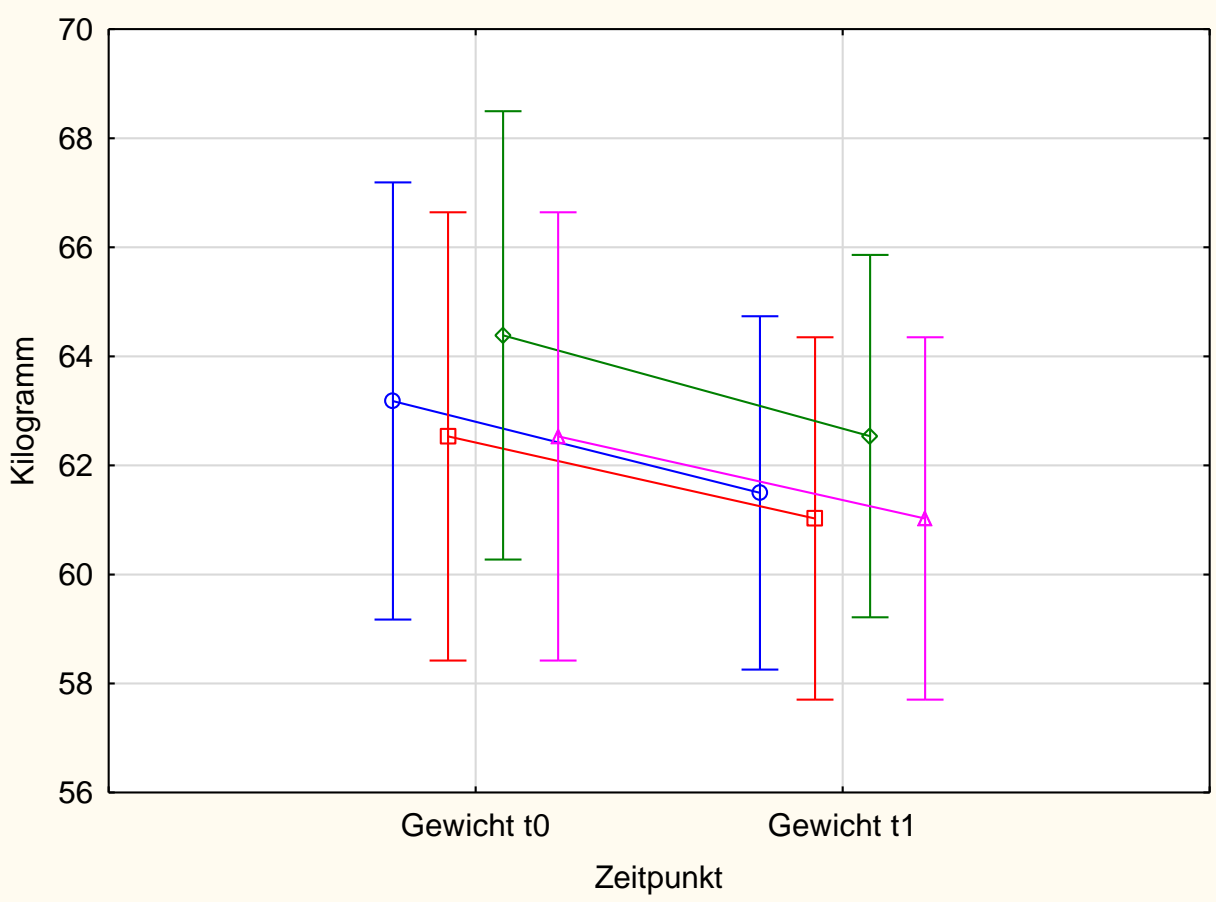

- Methode

DXA

$(n=$ jeweils 20$)$

西 Methode

$\mathrm{BIA}$

$(n=$ jeweils 19$)$

$\$$ Methode

Fettwaage

$(n=$ jeweils 19$)$

4 Methode

FUTREX

$(n=$ jeweils 19$)$

Abbildung 58: Mittelwert-Plot - Körpergewicht KETO (t0 - t1)

\section{Mittelwert-Plot - Körpergewicht LOGI (t0-t1)}

(Methodenvergleich innerhalb LOGl)

Interaktion von ,Zeitpunkt ${ }^{\star}$ Methode": $p=0,998$

(Vertikale Balken zeigen 0,95 Konfidenzintervalle)

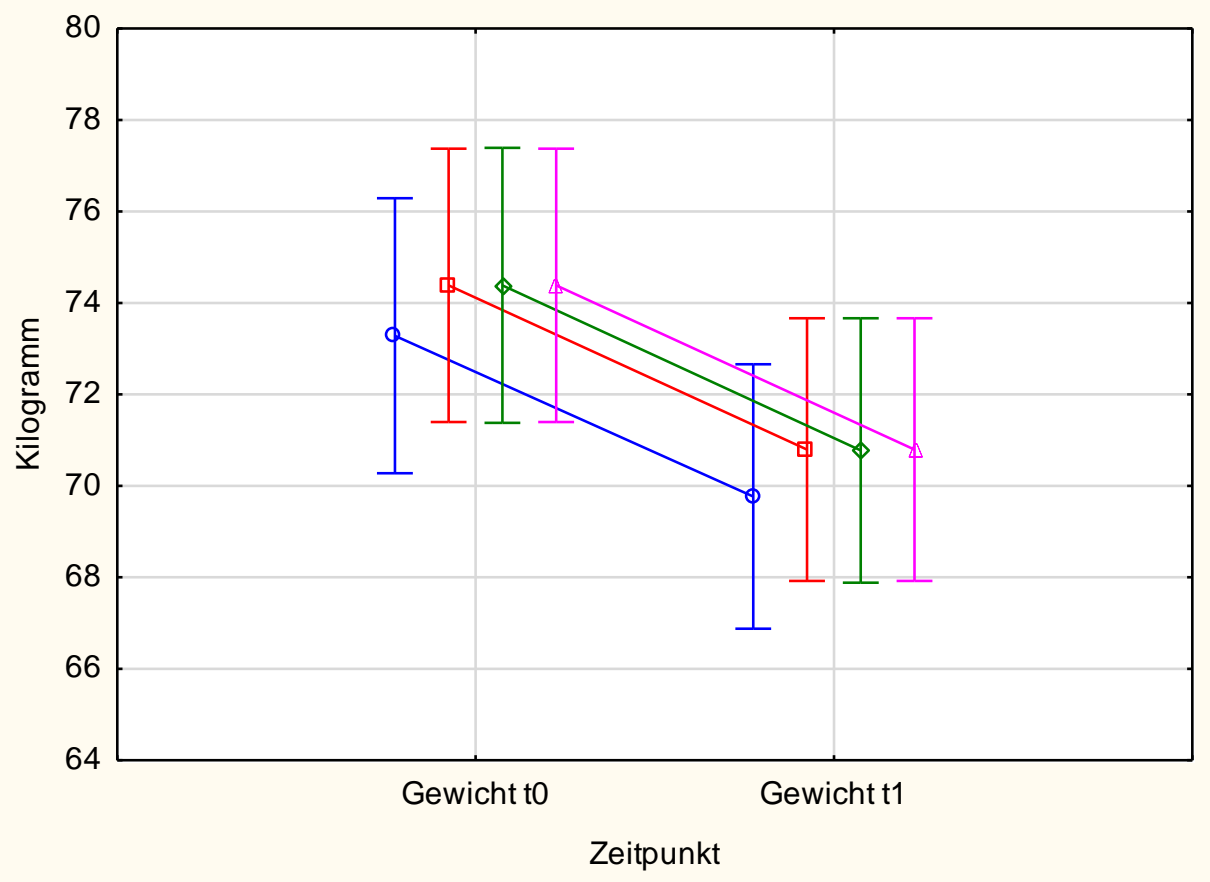

Methode DXA

$(n=$ jeweils 75$)$

刋 Methode

BIA

$(n=$ jeweils 76$)$

Methode

Fettwaage

$(n=$ jeweils 75$)$

4 Methode

FUTREX

$(n=$ jeweils 76$)$ 


\section{Mittelwert-Plot - Körpergewicht DGE (t0-t1)}

(Methodenvergleich innerhalb DGE)

Interaktion von ,Zeitpunkt* Methode": $p=0,998$

(Vertikale Balken zeigen 0,95 Konfidenzintervalle)

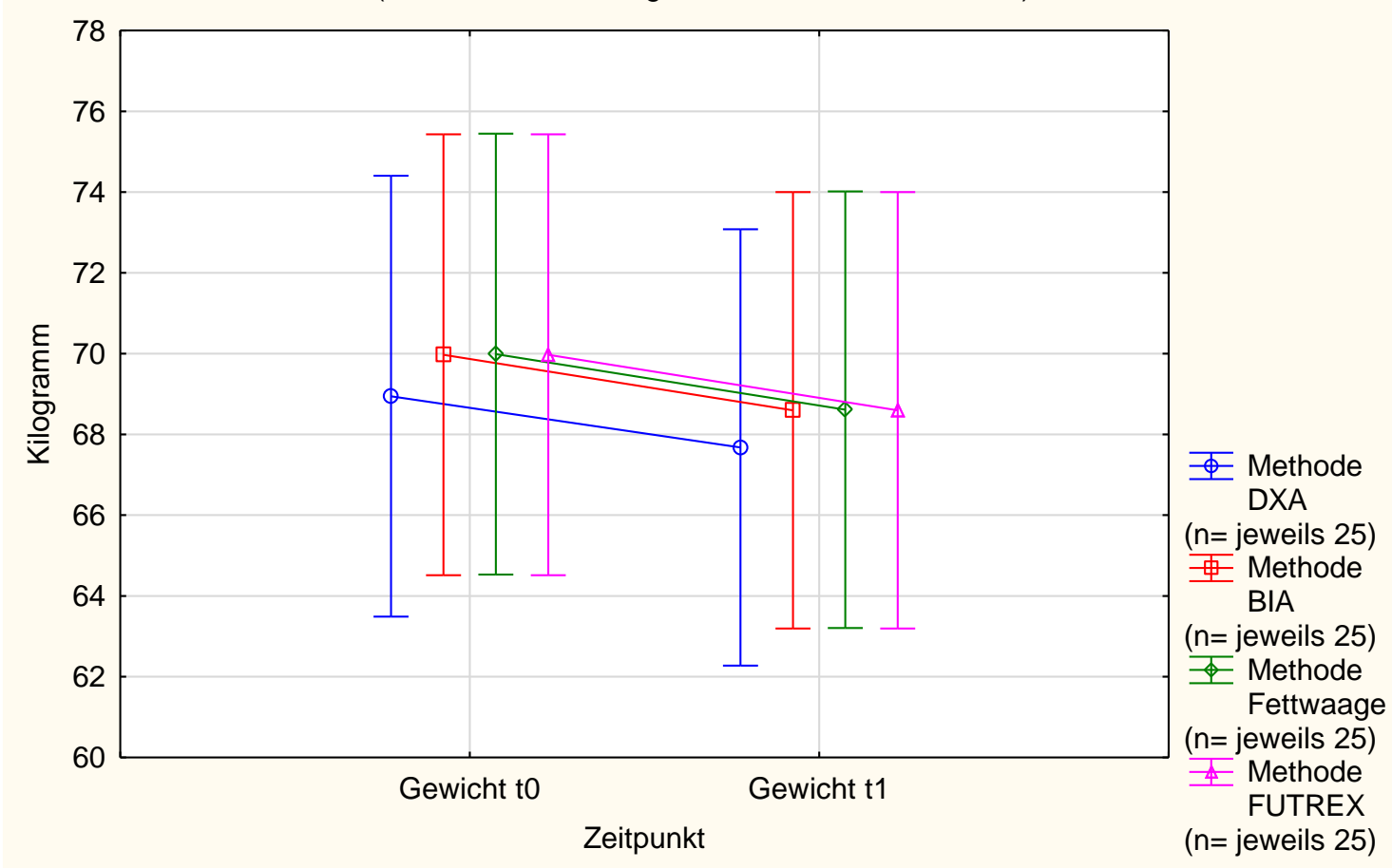

Abbildung 60: Mittelwert-Plot - Körpergewicht DGE (t0-t1)

Um die Änderungen über die Zeit (t0 - t1) zwischen den Methoden vergleichen zu können, wurde für jede Gruppe und Parameter eine einfaktorielle ANOVA erstellt und die Signifikanzwerte aus den jeweiligen Differenzen (t1 - t0) berechnet. Hierbei ergaben sich für das Körpergewicht keine signifikanten Unterschiede ( $p$-Wert hierfür innerhalb KETO=0,986; innerhalb $L O G I=0,986$ und innerhalb $D G E=0,977) . D . h$. in keiner der drei Gruppen unterschieden sich die durchschnittlichen Gewichtsverluste zwischen den Methoden signifikant voneinander.

\section{Fettmasse}

Bei der Betrachtung der durchschnittlichen Fettmassen im zeitlichen Verlauf (siehe Abbildung 61) fällt auf, dass wie beim Körpergewicht die Abnahme innerhalb LOGI am größten und im Vergleich zu KETO um mehr als das Zweifache, im Vergleich zu DGE sogar um beinahe das Dreieinhalbfache größer war. 


\section{Mittelwert-Plot - Fettmasse (t0-t1)}

(gemittelt über alle Messmethoden)

Interaktion von ,ZZeitpunktGruppe": $p<0,01$

(Vertikale Balken zeigen 0,95 Konfidenzintervalle)

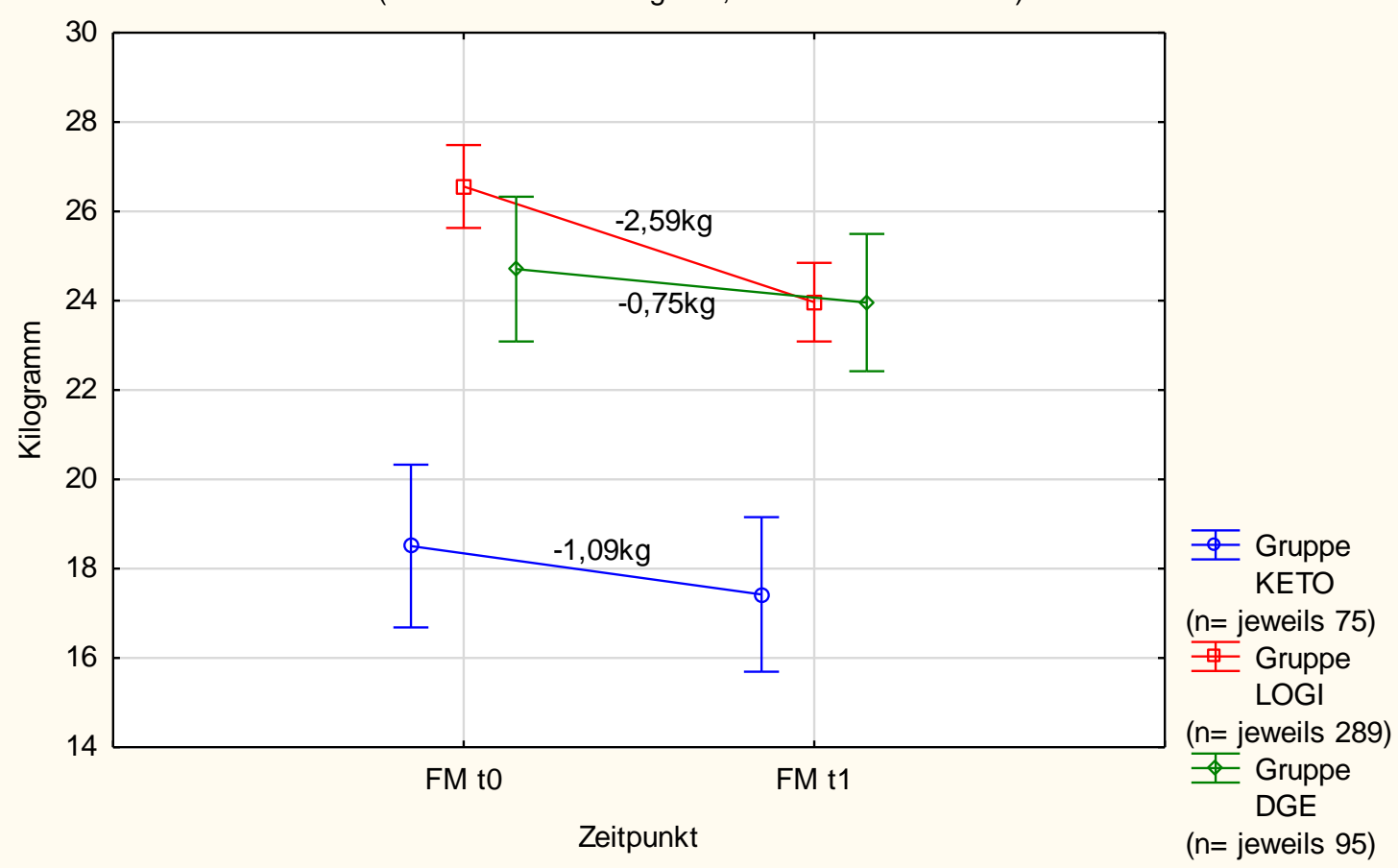

In den Abbildungen 62-64 sind die Verläufe der durchschnittlichen Fettmassen für jede Gruppe und Messmethode dargestellt.

Auch bei der Fettmasse gab es laut Varianzanalyse keine signifikanten Unterschiede bei der Betrachtung der Interaktion von „Zeitpunkt*Methode“ pro Gruppe. Analog zum Körpergewicht erbrachten nur die Post-hoc-Tests innerhalb LOGI signifikante Unterschiede, während innerhalb KETO und DGE keine zu finden waren.

Konkret bedeutet dies, dass innerhalb LOGI die Reduktion der Fettmasse im Verlauf von t0 bis t1 bei allen vier Methoden signifikant war ( $p$-Wert auch hier jeweils $<0,01$ ).

Im Vergleich der Methoden unterschieden sich bei keiner der drei Gruppen die Reduktionen der Fettmasse signifikant voneinander ( $p$-Wert innerhalb KETO=0,748; innerhalb $L O G I=0,698$ und innerhalb DGE=0,862). D. h. in keiner Gruppe maß eine Methode eine Abnahme der Fettmasse über die Zeit, die sich signifikant von einer anderen Methode unterschied. 


\section{Mittelwert-Plot - Fettmasse KETO (t0-t1)}

(Methodenvergleich innerhalb KETO)

Interaktion von ,Zeitpunkt*Methode": $\mathbf{p = 0 , 7 4 8}$

(Vertikale Balken zeigen 0,95 Konfidenzintervalle)

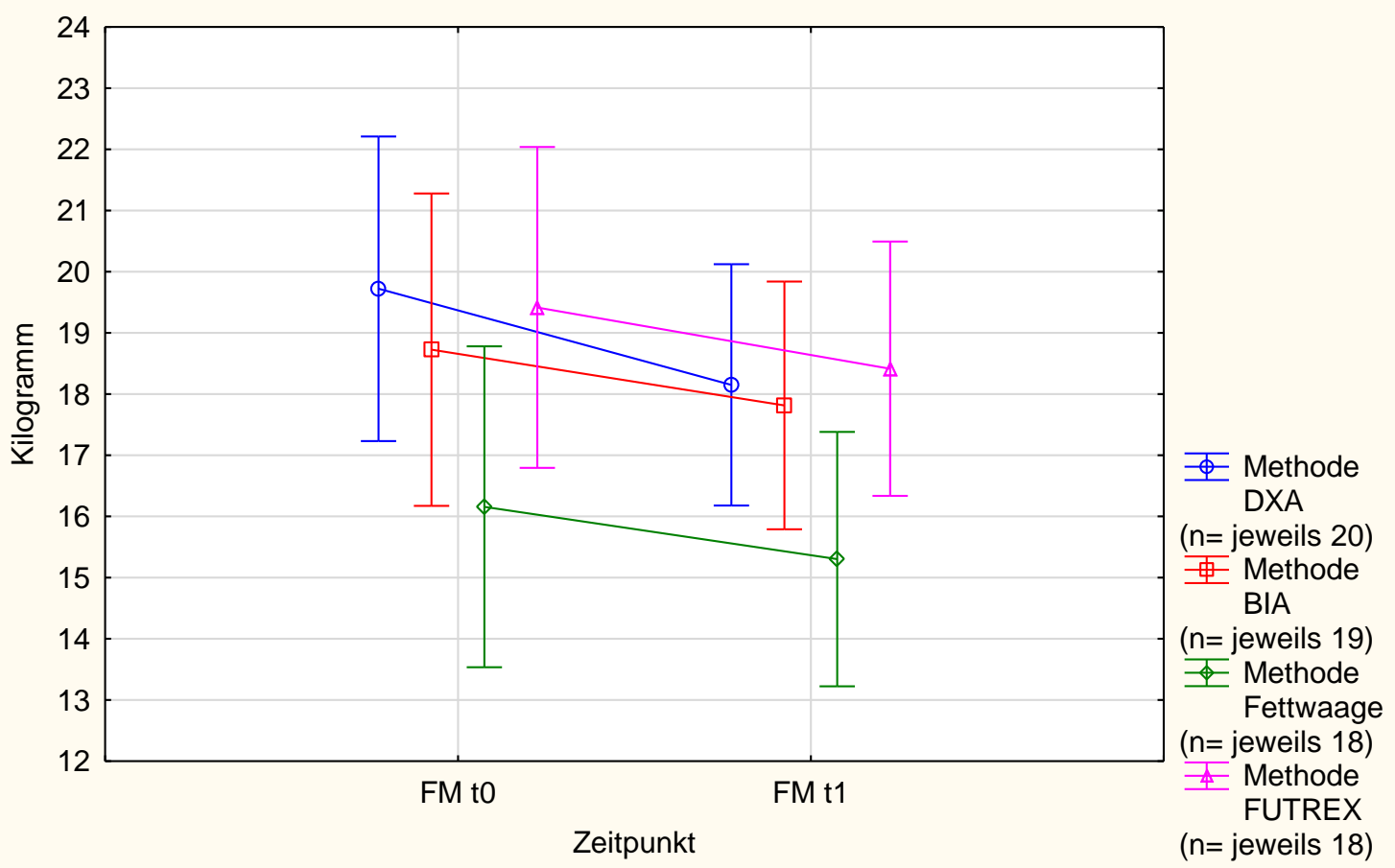

Abbildung 62: Mittelwert-Plot - Fettmasse KETO (t0-t1) 


\section{Mittelwert-Plot - Fettmasse LOGI (t0-t1)}

(Methodenvergleich innerhalb LOGI)

Interaktion von ,ZZeitpunkt*Methode": $p=0,698$

(Vertikale Balken zeigen 0,95 Konfidenzintervalle)

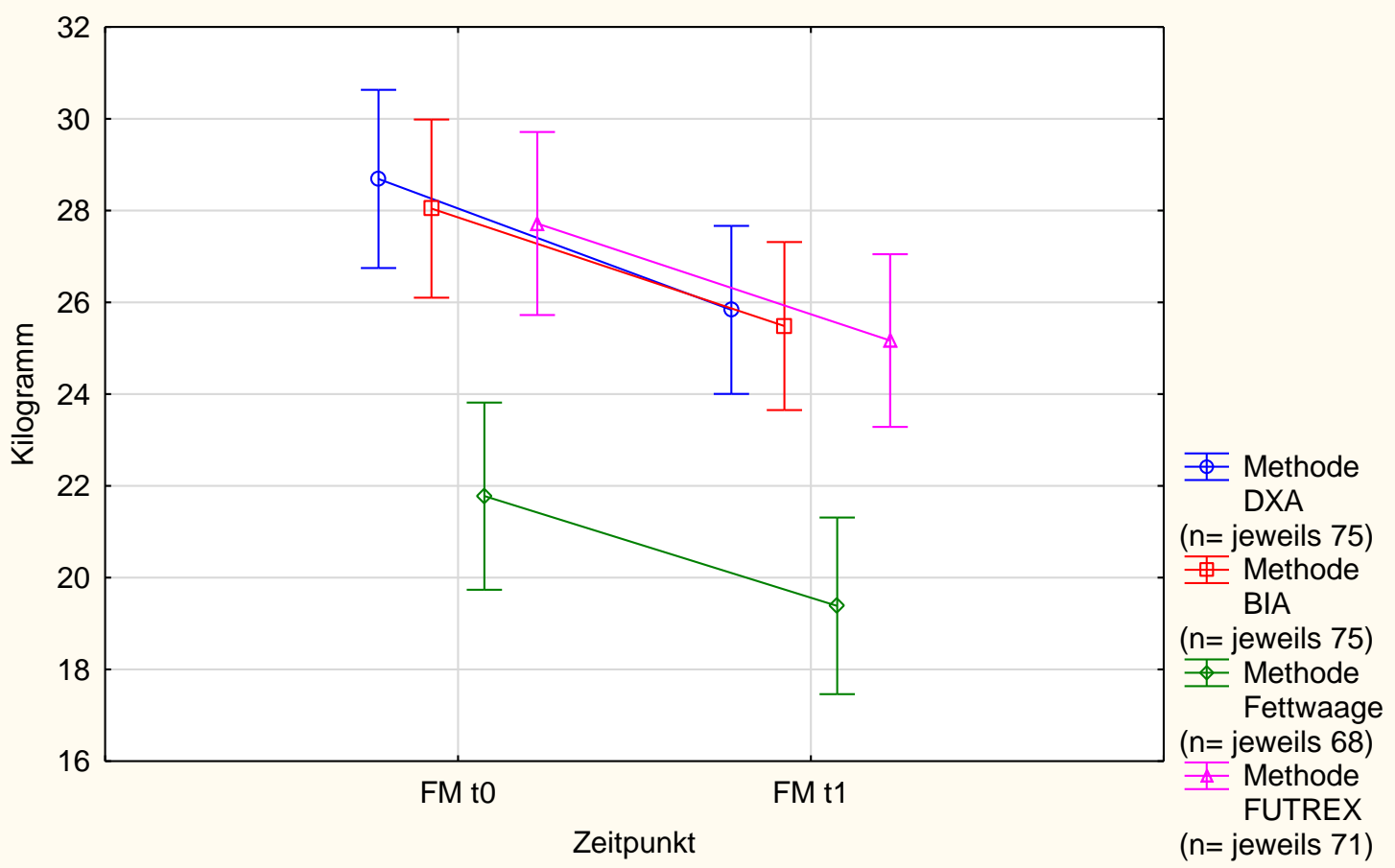

Abbildung 63: Mittelwert-Plot - Fettmasse LOGI (t0-t1) 


\section{Mittelwert-Plot - Fettmasse DGE (t0-t1)}

(Methodenvergleich innerhalb DGE)

Interaktion von ,Zeitpunkt*Methode": $p=\mathbf{0 , 6 4 5}$

(Vertikale Balken zeigen 0,95 Konfidenzintervalle)

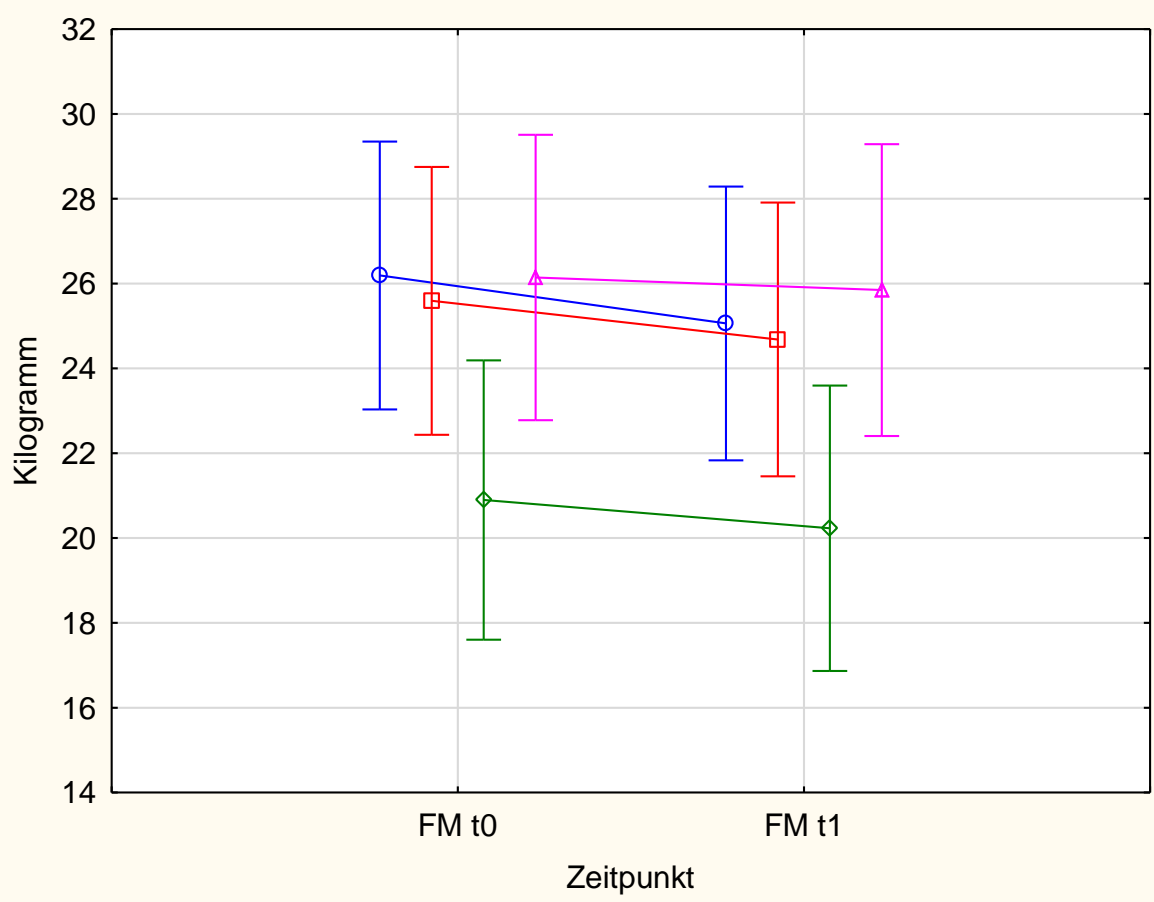

- Methode

DXA

( $n=$ jeweils 25)

平 Methode

BIA

( $n=$ jeweils 25)

$\$$ Methode

Fettwaage

$(n=$ jeweils 23)

Methode

FUTREX

$(n=$ jeweils 22)

Abbildung 64: Mittelwert-Plot - Fettmasse DGE (t0-t1)

\section{Fettfreie Masse}

In allen drei Gruppen war die durchschnittliche Abnahme an fettfreier Masse geringer als an Körpergewicht bzw. Fettmasse, die Tendenzen waren allerdings gleich (siehe Abbildung 65). Auch hier war die Abnahme innerhalb LOGI am größten, gefolgt von KETO und schließlich DGE. 


\section{Mittelwert-Plot - Fettfreie Masse (t0-t1)}

(gemittelt über alle Messmethoden)

Interaktion von ,ZZeitpunkt ${ }^{\star}$ Gruppe": $p<0,01$

(Vertikale Balken zeigen 0,95 Konfidenzintervalle)

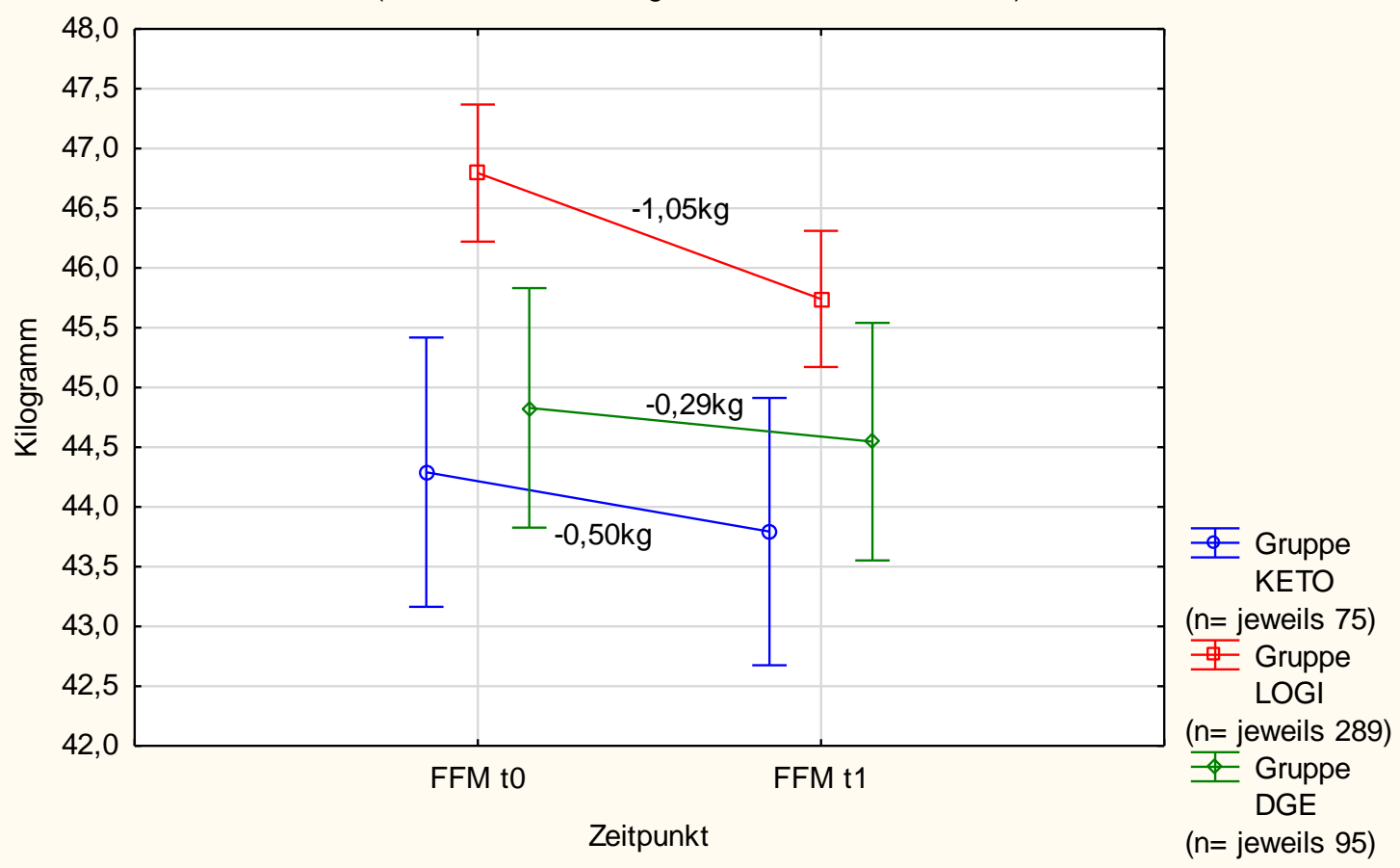

Abbildung 65: Mittelwert-Plot - Fettfreie Masse (t0-t1)

Auch hier wird nachfolgend der Verlauf der fettfreien Masse innerhalb jeder Gruppe für jede einzelne Methode in einem Mittelwert-Plot dargestellt (siehe Abbildung 66 - 68). Die p-Werte der Varianzanalysen lagen in jeder Gruppe zwar näher am Signifikanzniveau als beim Körpergewicht und der fettfreien Masse, unterschritten dieses allerdings auch hier nicht. Auch hier waren nur innerhalb LOGI signifikante Ergebnisse festzustellen. Allerdings mit dem Unterschied, dass hier die Reduktion der fettfreien Masse im Verlauf von t0 bis t1 nur bei den Messungen mittels BIA, Fettwaage und FUTREX signifikant ( $p$-Wert jeweils $<0,01)$ war, nicht aber bei der Messung mittels DXA $(p=0,174)$. Die Post-hoc-Tests innerhalb KETO und DGE erbrachten keinerlei signifikante Unterschiede. 


\section{Mittelwert-Plot - Fettfreie Masse KETO (t0-t1)}

(Methodenvergleich innerhalb KETO)

Interaktion von ,Zeitpunkt*Methode": $p=\mathbf{0 , 4 8 5}$

(Vertikale Balken zeigen 0,95 Konfidenzintervalle)

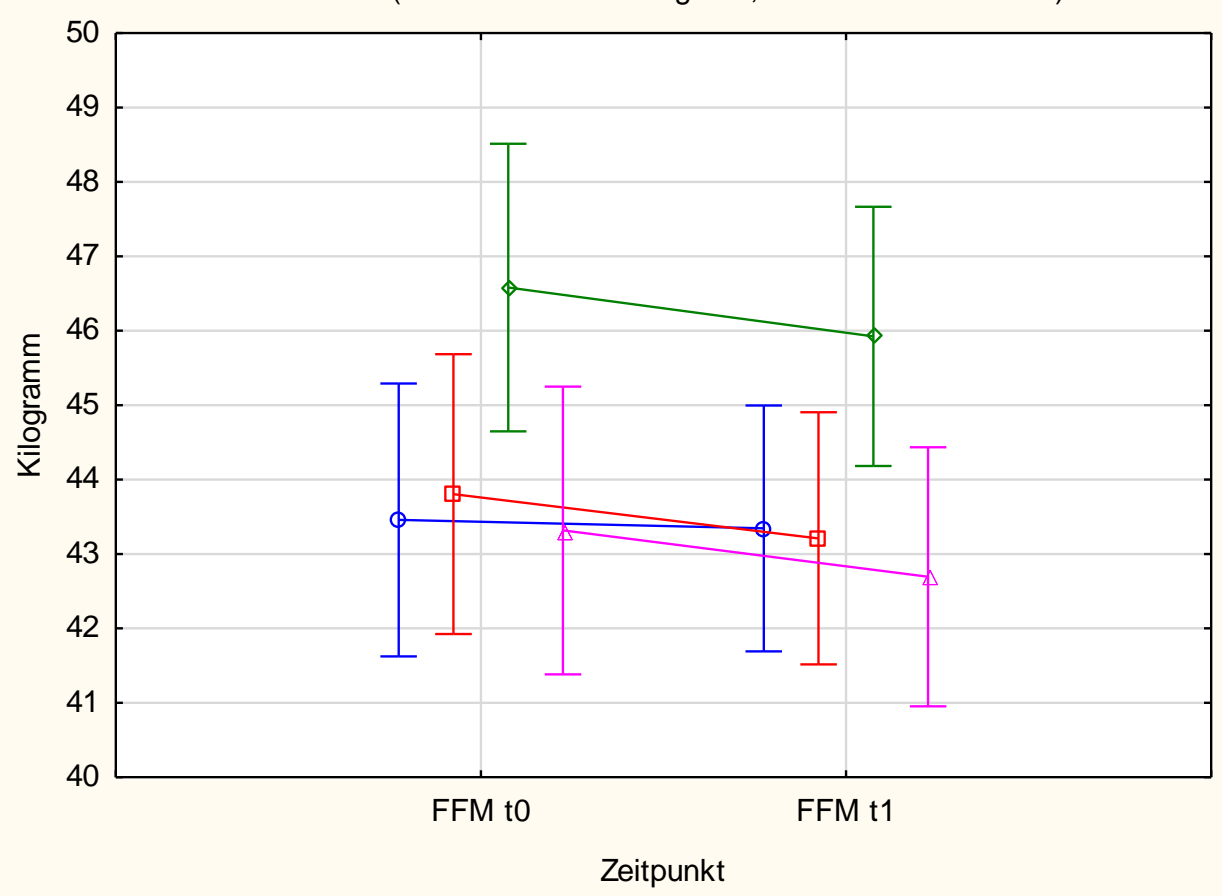

Methode

DXA

( $n=$ jeweils 20)

更 Methode

BIA

( $n=$ jeweils 19$)$

$\$$ Methode

Fettwaage

$(n=$ jeweils 18$)$

I Methode

FUTREX

$(n=$ jeweils 18$)$

\section{Mittelwert-Plot - Fettfreie Masse LOGI (t0-t1)}

(Methodenvergleich innerhalb LOGI)

Interaktion von ,ZZeitpunkt ${ }^{\star}$ Methode": $p=\mathbf{0 , 2 1 1}$

(Vertikale Balken zeigen 0,95 Konfidenzintervalle)

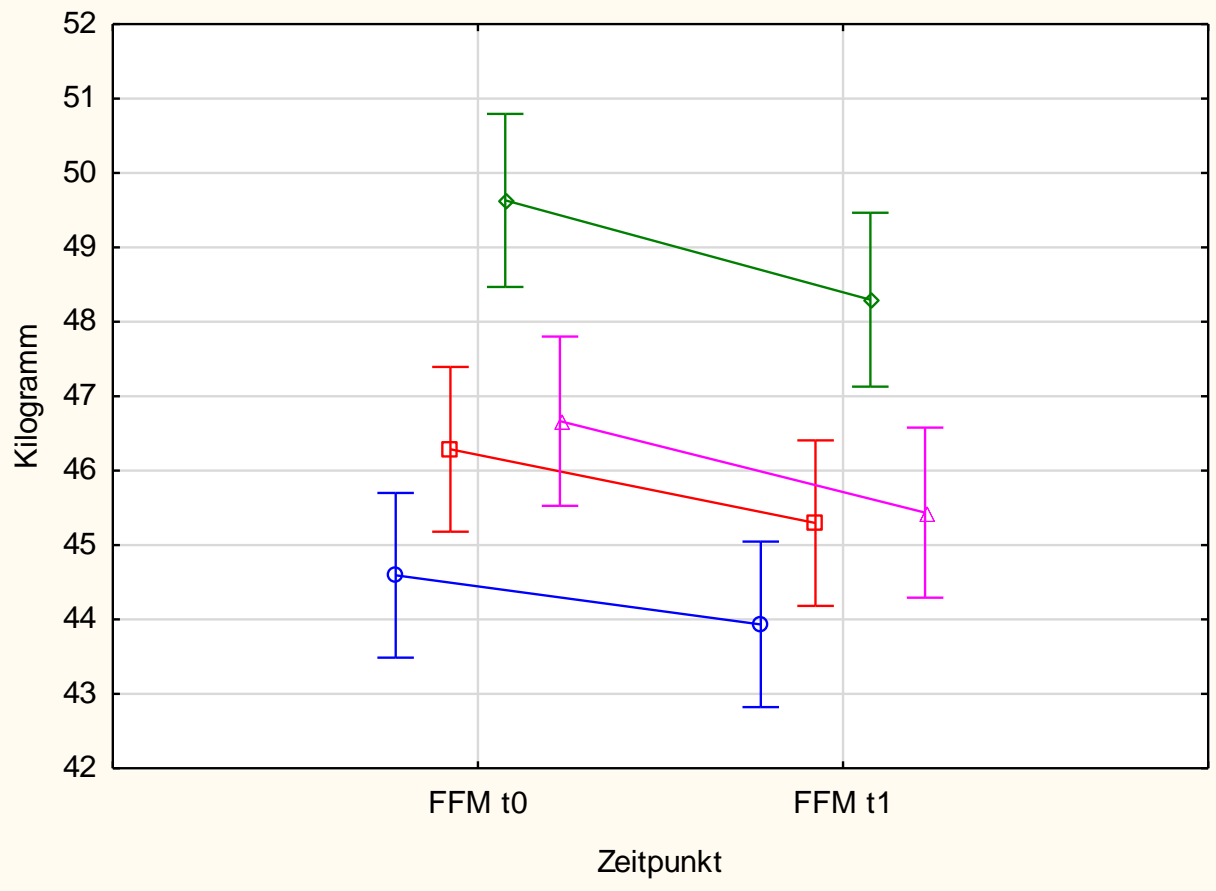

Methode DXA

$(n=$ jeweils 75$)$

西 Methode BIA

$(n=$ jeweils 75$)$

$\$$ Methode

Fettwaage $(n=$ jeweils 68 )

I Methode

FUTREX

$(n=$ jeweils 71$)$ 


\section{Mittelwert-Plot - Fettfreie Masse DGE (t0-t1)}

(Methodenvergleich innerhalb DGE) Interaktion von ,ZZeitpunkt ${ }^{\star}$ Methode": $p=\mathbf{0 , 4 2 2}$

(Vertikale Balken zeigen 0,95 Konfidenzintervalle)

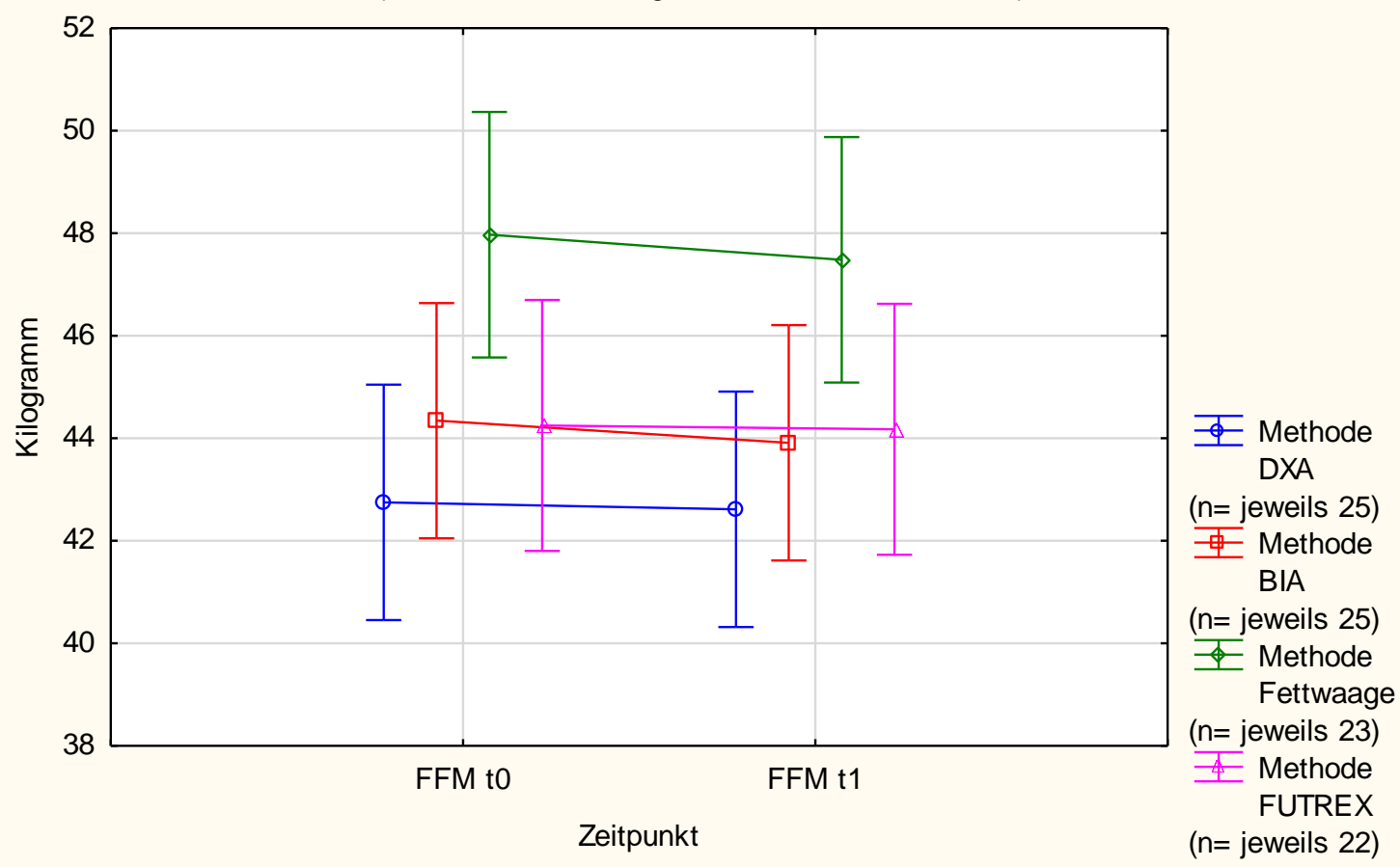

Abbildung 68: Mittelwert-Plot - Fettfreie Masse DGE (t0-t1)

Die Reduktionen der fettfreien Masse unterschieden sich auch hier im Methodenvergleich in keiner der drei Gruppen signifikant voneinander ( $p$-Wert hierfür innerhalb KETO=0,485; innerhalb LOGI $=0,118$ und innerhalb DGE=0,422). 


\subsection{Phasenwinkel}

Der Phasenwinkel ist sowohl zur Beurteilung des Ernährungs- als auch des Trainingszustandes geeignet (Data Input 2005), kann allerdings nur mittels BIA erhoben werden.

Bei der Betrachtung der Ergebnisse ist auch hier wieder darauf zu achten, dass sich die Anzahl (n) der Patientinnen zum Zeitpunkt to und im Verlauf unterschieden $(n(t))>$ $\mathrm{n}($ Verlauf)). Die Ergebnisse von t0 sind graphisch nicht dargestellt, sondern nur das Ergebnis der Varianzanalyse $(p(t 0))$.

Zum Zeitpunkt to fällt auf, dass die Patientinnen der KETO- und DGE-Gruppe durchschnittlich einen höheren - und gleichzeitig beinahe identischen $(p=1,0)$ - Phasenwinkel aufwiesen als die Patientinnen der LOGI-Gruppe (p(KETO-LOGI)=0,618; p(DGELOGII $=0,590)$. Für den Verlauf lässt sich sagen, dass die durchschnittlichen Veränderungen in allen drei Gruppen äußerst gering waren (KETO: +0,005; LOGI: +0,014; DGE: 0,022 ) und sich zwischen den Gruppen nicht signifikant voneinander unterschieden (siehe Abbildung 69).

\section{Mittelwert-Plot - Phasenwinkel (t0-t1)}

$p(t 0)=0,269$

$p($ Verlauf $)=0,869$

(Vertikale Balken zeigen 0,95 Konfidenzintervalle)

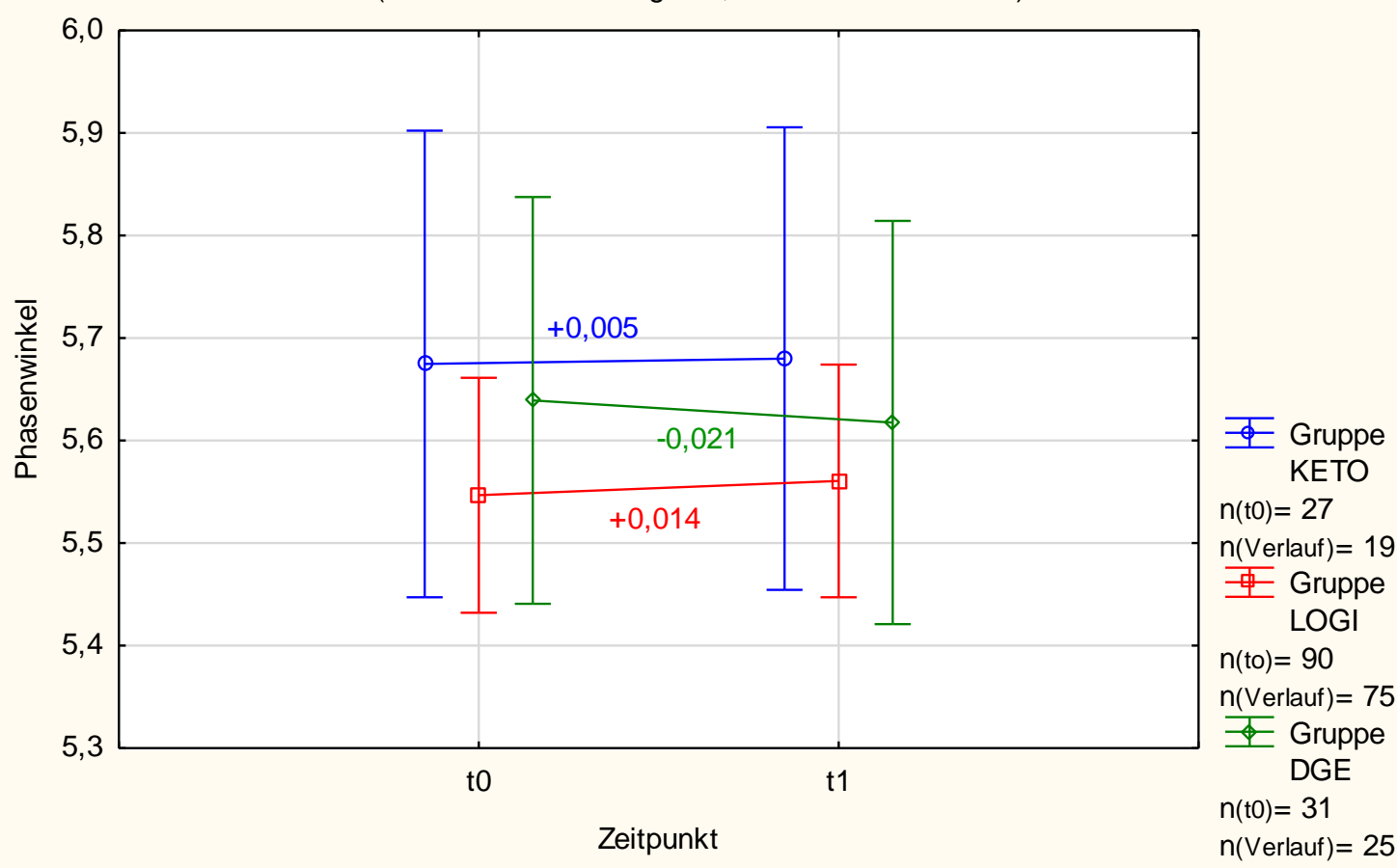




\subsection{Spiroergometrie}

In den Legenden der Mittelwert-Plots sind unterschiedliche Patientinnen-Anzahlen bei $n(t 0)$ und $n($ Verlauf) angegeben. Dies ist zum einen darauf zurückzuführen, dass auch hier für die Verlaufsbetrachtung nur Patientinnen bzw. deren Messergebnisse berücksichtigt werden konnten, die zu beiden Zeitpunkten t0 und t1 an der Untersuchung teilgenommen haben (Gründe für das Ausscheiden aus der Studie: siehe 1.2 Ablauf der Studie und Patientenrekrutierung) und zum anderen aber auch darauf, dass einzelne Parameter nicht erhoben wurden/werden konnten. Die Gründe hierfür werden bei den jeweiligen Parametern erläutert.

Um die Darstellung weiterer Graphen zu vermeiden, wurden die Ergebnisse von t0 auch hier nicht zusätzlich graphisch dargestellt, sondern lediglich die berechneten $\mathrm{p}$ Werte als $p(t 0)$ angegeben (diese beziehen sich auf $n(t 0)$ ). Hierbei fällt auf, dass sich zum Zeitpunkt t0 für alle Parameter außer beim Laktat 3 min. nach Belastung signifikante Gruppenunterschiede ergaben. Dies war zwischen KETO und den anderen beiden Gruppen fast immer der Fall (außer zwischen KETO-LOGI bei Zeit bis zum Ende der Belastung) während zwischen LOGI und DGE insgesamt nur ein signifikanter Unterschied gefunden werden konnte. Zur besseren Übersicht sind die Signifikanzwerte der Post-hoc-Tests bei to für alle Parameter in Tabelle 4 zusammengefasst und werden bei der Ergebnisbeschreibung der einzelnen Parameter nicht mehr erwähnt.

\section{$\underline{\text { RER in Ruhe }}$}

Die geringere Anzahl an Messwerten im Vergleich zu den anderen Parametern lässt sich dadurch erklären, dass die Messwerte der 10-minütigen Ruhephase vor der eigentlichen Durchführung der Spiroergometrie bei vielen Patientinnen aus technischen Gründen nicht gespeichert wurden/werden konnten und deshalb die Errechnung des Ruheumsatzes nicht möglich war.

Im entsprechenden Mittelwert-Plot (siehe Abbildung 70) ist zu sehen, dass zu beiden Zeitpunkten die Patientinnen der KETO-Gruppe durchschnittlich die niedrigsten und die der DGE-Gruppe dagegen die höchsten Werte aufwiesen. Der Mittelwert war hierbei im Verlauf innerhalb KETO am meisten gesunken, während es innerhalb LOGI und DGE keine nennenswerten Veränderungen gab (+ 0,005 bzw. - 0,004). 
Tabelle 4: Signifikanzwerte der Post-hoc-Tests bei der Spiroergometrie zum Zeitpunkt t0

\begin{tabular}{|c|c|c|c|}
\hline Zeitpunkt t0 & \multicolumn{3}{|c|}{ Signifikante Unterschiede zwischen den Gruppen } \\
\hline Parameter & KETO - LOGI & KETO - DGE & LOGI - DGE \\
\hline RER in Ruhe & $P<0,01$ & $P<0,01$ & $P=0,287$ \\
\hline VO2/kg bei VT2 & $P<0,01$ & $P<0,01$ & $P=0,134$ \\
\hline VO2/kgMAX & $P<0,01$ & $P<0,01$ & $P=0,113$ \\
\hline Last bei VT2 & $P<0,01$ & $P<0,01$ & $P=0,065$ \\
\hline LastMAX & $P=0,027$ & $P<0,01$ & $P=0,072$ \\
\hline $\begin{array}{l}\text { Zeit bis zum Ende } \\
\text { der Belastung }\end{array}$ & $P=0,061$ & $P<0,01$ & $P=0,036$ \\
\hline $\begin{array}{l}\text { Laktat } 3 \text { min. nach } \\
\text { Belastung }\end{array}$ & \multicolumn{3}{|c|}{ Keine signifikanten Unterschiede } \\
\hline
\end{tabular}

Legende 1: RER in Ruhe = Respiratory exchange ratio in Ruhe/Ruheumsatzbestimmung

VO2/kg bei VT2 = Relative VO2 (körpergewichtsbezogene Angabe der VO2) am sog. respiratorischen Kompensationspunkt ( $\hat{=}$ Ende des aerob-anaeroben-Übergangs)

$\mathrm{VO} 2 / \mathrm{kg} \mathrm{MAX}=$ Maximal erreichte $\mathrm{VO} 2 / \mathrm{kg}$

Last bei VT2 = Leistung [Watt] am resp. Kompensationspunkt

Last MAX = Maximal erreichte Leistung [Watt]

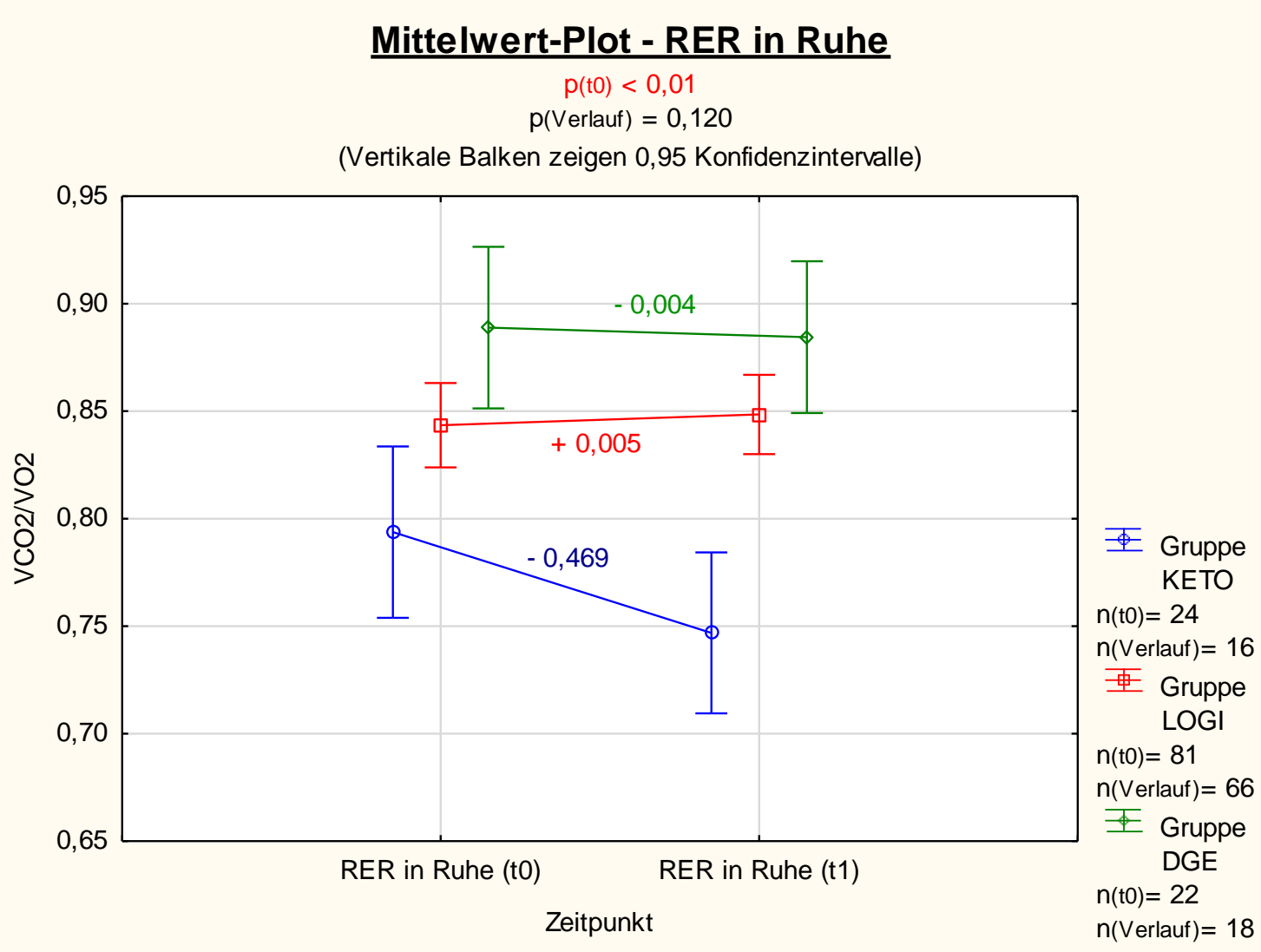

Abbildung 70: Mittelwert-Plot - Ruheumsatzbestimmung 


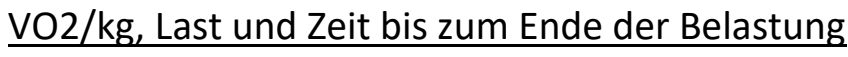

Für die unterschiedlichen Patientinnen-Anzahlen gab es verschiedene Gründe. Bei t0 kam es innerhalb der LOGI-Gruppe bei vier Patientinnen zu technischen Problemen bei der Durchführung der Spiroergometrie, sodass keine bzw. keine plausiblen Messwerte erzielt werden konnten, zwei Patientinnen hatten die Studie unmittelbar vor der Durchführung der Spiroergometrie abgebrochen und zwei Patientinnen konnten aus gesundheitlichen Gründen nicht teilnehmen. Innerhalb der DGE-Gruppe traten bei einer Patientin technische Probleme auf und eine weitere Patientin konnte aus gesundheitlichen Gründen nicht teilnehmen. Im Gegensatz dazu konnte die Spiroergometrie innerhalb der KETO-Gruppe bei allen Patientinnen durchgeführt werden.

Neben dem Ausscheiden aus der Studie waren die Gründe für die geringere Anzahl an Patientinnen bei 11 fast ausschließlich technischer Natur. Entweder waren die ermittelten Messwerte unplausibel, sodass es zu Fehlern während der Messung gekommen sein musste, oder die ermittelten Daten konnten nach der Durchführung nicht übertragen/gespeichert werden und standen dementsprechend nicht zur Auswertung zu Verfügung.

Bei der Betrachtung der Leistungsparameter VO2/kg (siehe Abbildung 71 und 72) und Last (siehe Abbildung 73 und 74) fällt auf, dass die Patientinnen der KETO-Gruppe zu beiden Zeitpunkten durchschnittlich die höchsten Werte und die Patientinnen der DGE-Gruppe durchschnittlich die niedrigsten Werte erreichten. Im Verlauf konnten die Patientinnen der KETO-Gruppe ihre Werte bei VT2 deutlich mehr verbessern als die anderen beiden Gruppen, bei den erreichten Maximalwerten der beiden Parameter war dieser Unterschied allerdings nicht so groß und bei "Last MAX“ war der durchschnittliche Anstieg bei DGE sogar am größten.

Eine weitere Möglichkeit der Darstellung und Beurteilung der Parameter VO2/kg MAX und Last MAX zu beiden Zeitpunkten - neben den Mittelwert-Plots - ist der Vergleich mit vorhandenen Sollwerten aus der Literatur. Kroidl et al. (2010) empfehlen für die Ergebnisse der VO2/kg MAX eine Einteilung in Fitnesskategorien nach Cooper und für die maximale Leistung (Last MAX) wie bereits erwähnt die Orientierung an den WattSollwerten nach dem American College of Sports Medicine (ACSM). Die entsprechenden Ergebnisse für jeweils beide Zeitpunkte t0 und t1 sind in den Tabellen 5 bis 8 dargestellt. 


\section{Mittelwert-Plot - VO2/kg bei VT2}

$\mathrm{p}(\mathrm{t} 0)<0,01$

$p($ Verlauf $)=0,116$

(Vertikale Balken zeigen 0,95 Konfidenzintervalle)

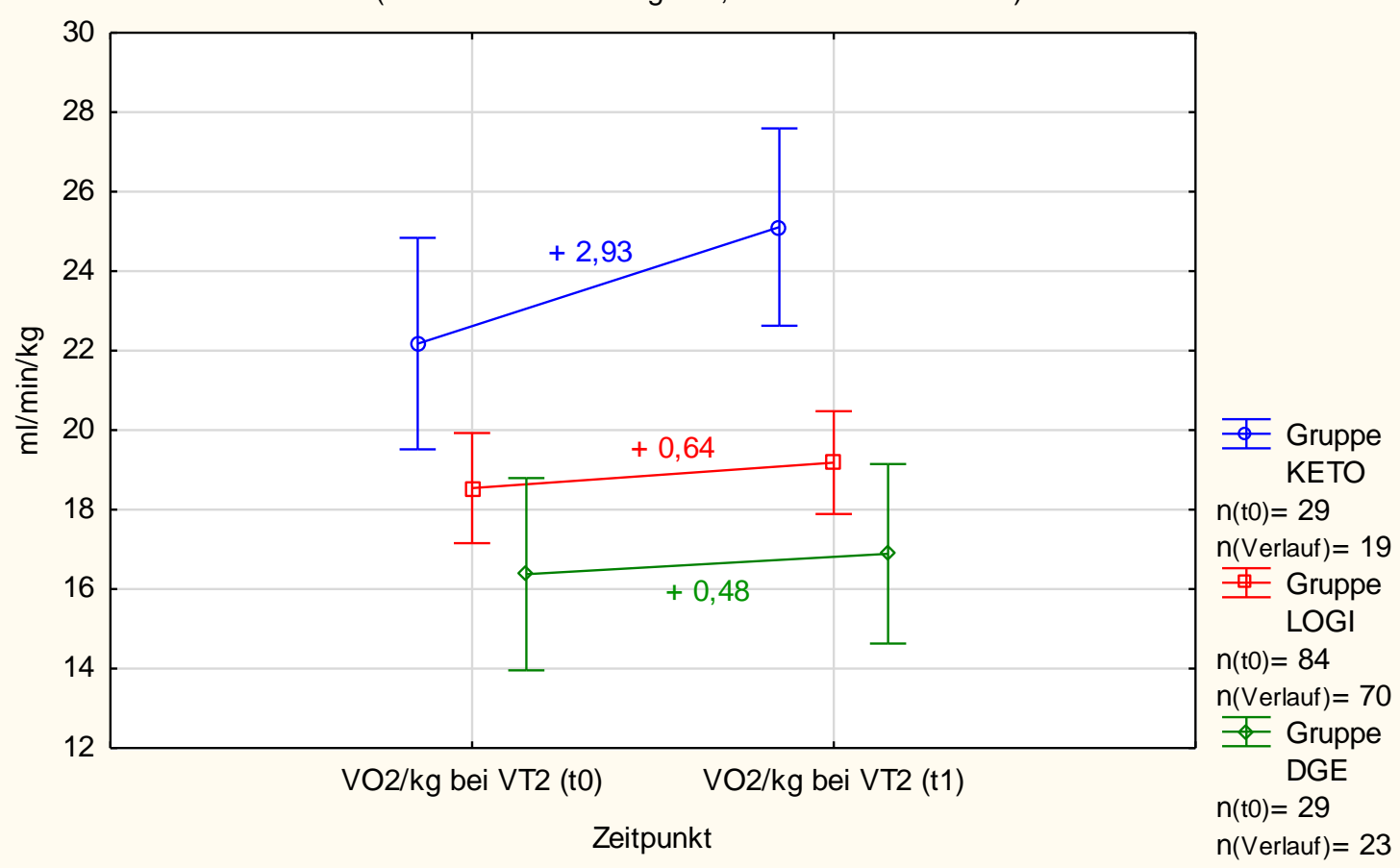

Abbildung 71: Mittelwert-Plot - VO2/kg bei VT2 


\section{Mittelwert-Plot - VO2/kg MAX}

$\mathrm{p}(\mathrm{t} 0)<0,01$

$p($ Verlauf $)=0,277$

(Vertikale Balken zeigen 0,95 Konfidenzintervalle)

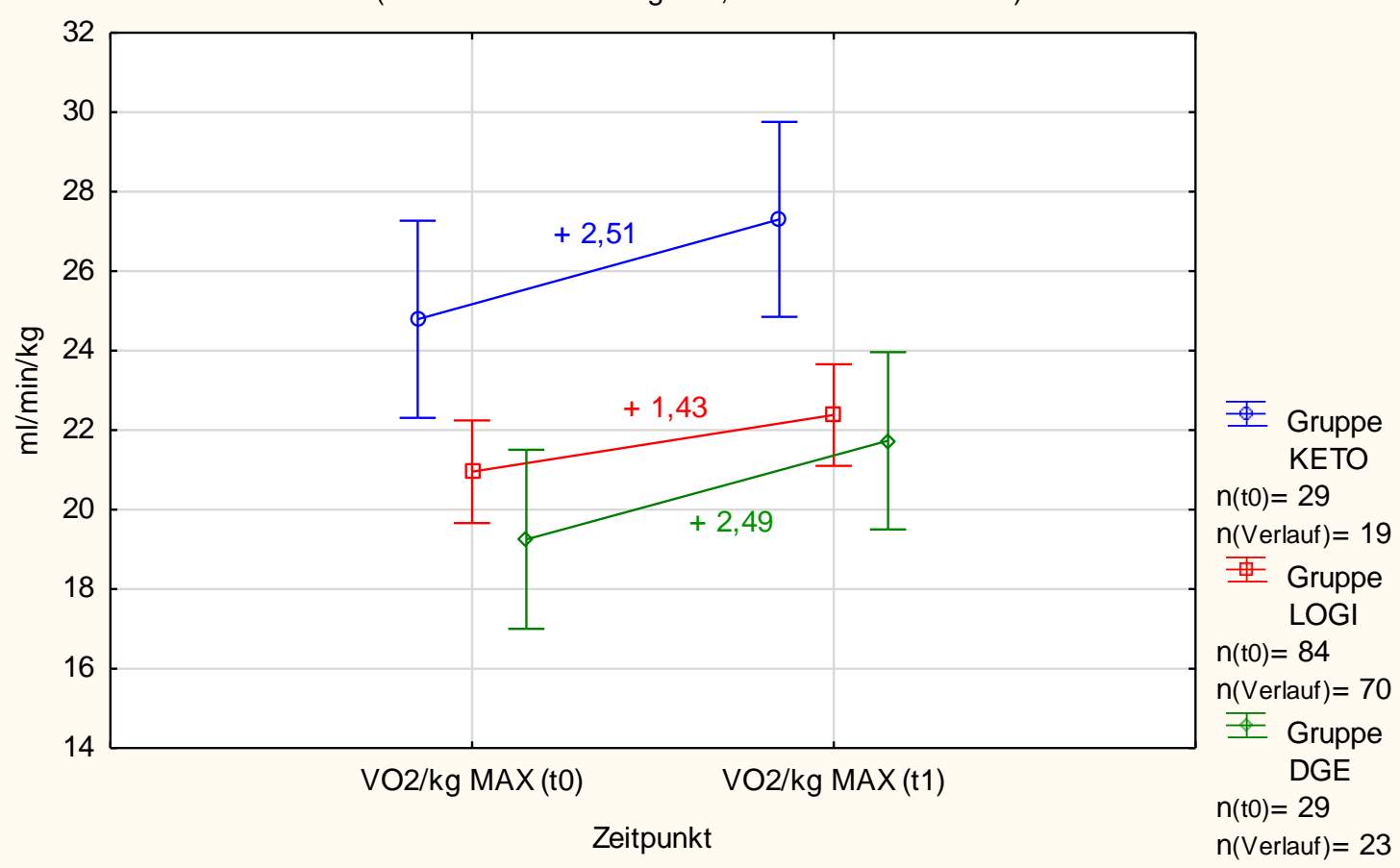

Abbildung 72: Mittelwert-Plot - VO2/kg MAX 
Mittelwert-Plot - Last bei VT2

$\mathrm{p}(\mathrm{t0})<0,01$

$p($ Verlauf $)=0,457$

(Vertikale Balken zeigen 0,95 Konfidenzintervalle)

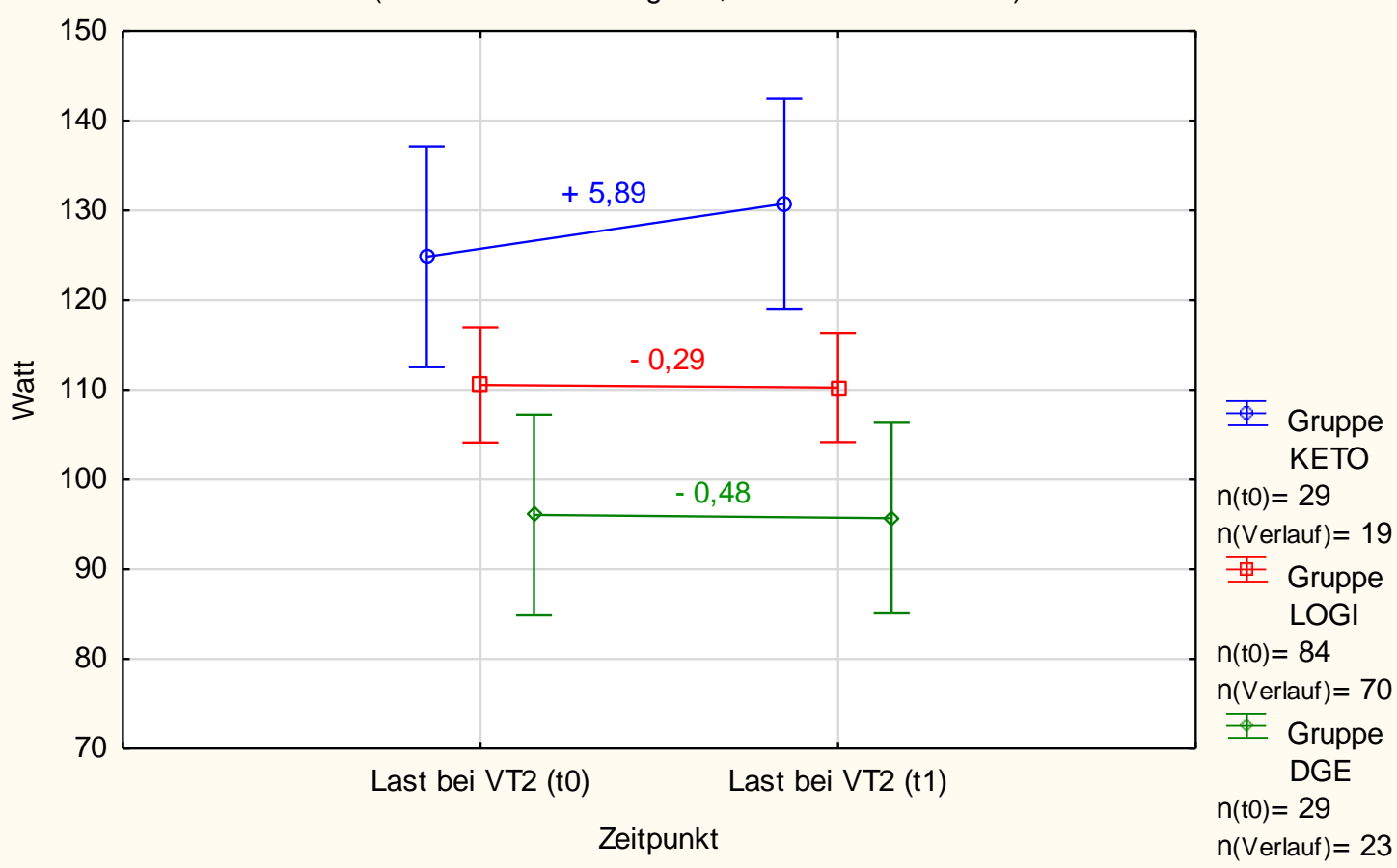

Abbildung 73: Mittelwert-Plot - Last bei VT2 


\section{Mittelwert-Plot - Last MAX}

$\mathrm{p}(\mathrm{t} 0)<0,01$

$p($ Verlauf $)=0,413$

(Vertikale Balken zeigen 0,95 Konfidenzintervalle)

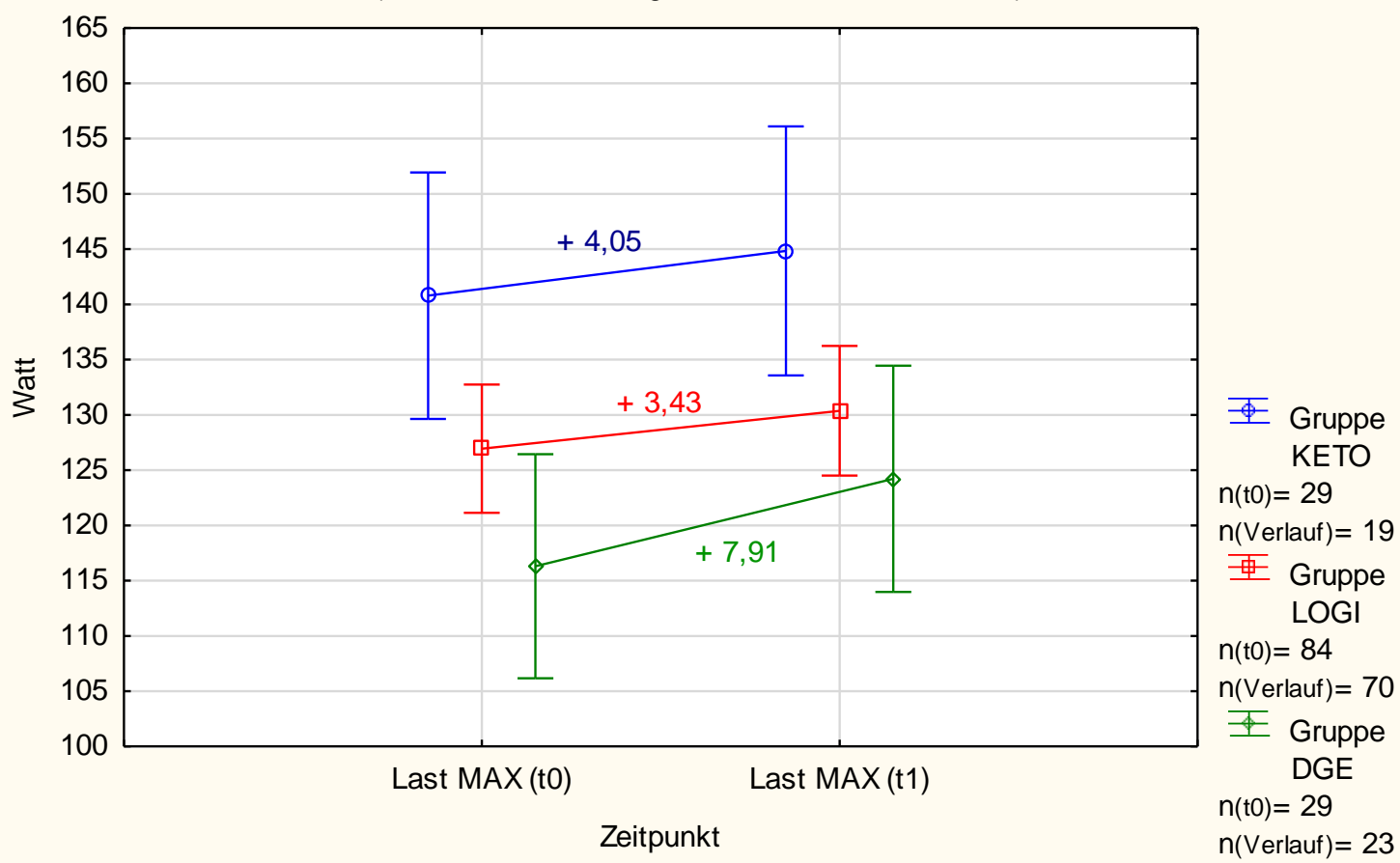

Abbildung 74: Mittelwert-Plot - Last MAX 
Tabelle 5: Erreichte Fitnesskategorien nach Cooper zum Zeitpunkt t0 (Kroidl et al. 2010)

\begin{tabular}{|l|c|c|c|c|c|c|c|c|c|c|c|}
\hline Gruppe & $\begin{array}{c}\text { Anzahl Pat. } \\
\text { Gesamt }\end{array}$ & $\begin{array}{c}\text { Kategorie 1 } \\
\text { (niedrig) }\end{array}$ & Prozent & $\begin{array}{c}\text { Kategorie 2 } \\
\text { (mäßig) }\end{array}$ & Prozent & $\begin{array}{c}\text { Kategorie 3 } \\
\text { (mittel) }\end{array}$ & Prozent & $\begin{array}{c}\text { Kategorie 4 } \\
\text { (gut) }\end{array}$ & $\begin{array}{c}\text { Prozent } \\
\text { (hoch) }\end{array}$ & $\begin{array}{c}\text { Kategorie 5 } \\
\text { Prozent }\end{array}$ \\
\hline KETO & 29 & 6 & $20,69 \%$ & 8 & $27,59 \%$ & 9 & $31,03 \%$ & 3 & $10,34 \%$ & 3 & $10,34 \%$ \\
LOGI & 84 & 31 & $36,90 \%$ & 31 & $36,90 \%$ & 18 & $21,43 \%$ & 3 & $3,57 \%$ & 1 \\
DGE & 29 & 16 & $55,17 \%$ & 8 & $27,59 \%$ & 4 & $13,79 \%$ & 1 & $3,45 \%$ & 0 & $0,00 \%$ \\
\hline
\end{tabular}

Tabelle 6: Erreichte Fitnesskategorien nach Cooper zum Zeitpunkt t1 (Kroidl et al. 2010)

\begin{tabular}{|c|c|c|c|c|c|c|c|c|c|c|c|}
\hline Gruppe & $\begin{array}{c}\text { Anzahl Pat. } \\
\text { Gesamt }\end{array}$ & $\begin{array}{c}\text { Kategorie } 1 \\
\text { (niedrig) }\end{array}$ & Prozent & $\begin{array}{c}\text { Kategorie } 2 \\
\text { (mäßig) }\end{array}$ & Prozent & $\begin{array}{c}\text { Kategorie } 3 \\
\text { (mittel) }\end{array}$ & Prozent & $\begin{array}{c}\text { Kategorie } 4 \\
\text { (gut) }\end{array}$ & Prozent & $\begin{array}{c}\text { Kategorie } 5 \\
\text { (hoch) }\end{array}$ & Prozent \\
\hline KETO & 19 & 2 & $10,53 \%$ & 5 & $26,32 \%$ & 5 & $26,32 \%$ & 2 & $10,53 \%$ & 5 & $26,32 \%$ \\
\hline LOGI & 70 & 21 & $30,00 \%$ & 21 & $30,00 \%$ & 20 & $28,57 \%$ & 6 & $8,57 \%$ & 2 & $2,86 \%$ \\
\hline DGE & 23 & 8 & $34,78 \%$ & 8 & $34,78 \%$ & 5 & $21,74 \%$ & 1 & $4,35 \%$ & 1 & $4,35 \%$ \\
\hline
\end{tabular}

Tabelle 7: Vergleich der Ergebnisse für Last MAX mit den Sollwerten für die maximale Leistung [Watt] unter Bezug auf das Alter und Körpergewicht nach dem ACSM zum Zeitpunkt to (Kroidl et al. 2010)

\begin{tabular}{|l|c|c|c|c|c|c|c|}
\hline Gruppe & $\begin{array}{c}\text { Anzahl Pat. } \\
\text { Gesamt }\end{array}$ & Sollwerte erreicht & Prozent & Sollwert nicht erreicht & Prozent & $\begin{array}{c}\text { Gewichtsgrenze } \\
\text { (89kg) überschritten }\end{array}$ & Prozent \\
\hline KETO & 29 & 23 & $79,31 \%$ & 5 & $17,24 \%$ & 1 & $3,45 \%$ \\
LOGI & 84 & 41 & $48,81 \%$ & 34 & $40,48 \%$ & 9 & $10,71 \%$ \\
DGE & 29 & 11 & $51,72 \%$ & 15 & $37,93 \%$ & 3 & $10,35 \%$ \\
\hline
\end{tabular}

Tabelle 8: Vergleich der Ergebnisse für Last MAX mit den Sollwerten für die maximale Leistung [Watt] unter Bezug auf das Alter und Körpergewicht nach dem ACSM zum Zeitpunkt t1 (Kroidl et al. 2010)

\begin{tabular}{|l|c|c|c|c|c|c|c|}
\hline Gruppe & $\begin{array}{c}\text { Anzahl Pat. } \\
\text { Gesamt }\end{array}$ & Sollwerte erreicht & Prozent & Sollwert nicht erreicht & Prozent & $\begin{array}{c}\text { Gewichtsgrenze } \\
\text { (89kg) überschritten }\end{array}$ & Prozent \\
\hline KETO & 19 & 18 & $94,74 \%$ & 1 & $5,26 \%$ & 0 & $0,00 \%$ \\
LOGI & 70 & 43 & $61,43 \%$ & 20 & $28,57 \%$ & 7 & $10,00 \%$ \\
DGE & 23 & 12 & $52,17 \%$ & 7 & $30,43 \%$ & 4 & $17,40 \%$ \\
\hline
\end{tabular}


Bei den Fitnesskategorien ist hervorzuheben, dass der prozentuale Anteil in den beiden schlechtesten Kategorien 1 und 2 zum Zeitpunkt tO innerhalb der KETO-Gruppe weniger als $50 \%$ betrug, während dieser innerhalb LOGI mit mehr als 70\% und innerhalb DGE mit sogar mehr als $80 \%$ jeweils deutlich größer war. Zudem konnten mehr als 10\% der Patientinnen der KETO-Gruppe die höchste Kategorie 5 erreichen, während dies innerhalb LOGI nur bei 1\% der Fall war und innerhalb DGE keine einzige Patientin diese Kategorie erreichen konnte.

Im Verlauf ist deutlich zu sehen, dass der prozentuale Anteil der schlechtesten beiden Kategorien in allen drei Gruppen zugunsten der besseren Kategorien abgenommen hatte. Bei KETO fällt auf, dass sich der Anteil aus der Kategorie 1 beinahe halbierte, es bei den mittleren Kategorien 2 - 4 nur wenig Änderungen gegeben hatte und der Anteil an Kategorie 5 dafür um mehr als das doppelte angestiegen war. Bei LOGI und DGE war der prozentuale Abfall in der Kategorie 1 ähnlich groß und in Kategorie 2 sogar größer als bei KETO. Allerdings wuchs bei ihnen nur der Anteil an Kategorie 3 und 4 deutlich an, sodass der Anteil an Kategorie 5 innerhalb KETO im Vergleich zu LOGI und DGE sogar noch größer war als zu Beginn (t0).

Passend zu den Ergebnissen der Einteilung in die Fitnesskategorien stellte sich der Vergleich der Ergebnisse für die maximal erreichte Last mit den Sollwerten nach dem ACSM dar. Bei to konnten die körpergewichtsbezogenen Sollwerte bei fast $80 \%$ der Patientinnen der KETO-Gruppe erreicht werden, während dieser Anteil innerhalb LOGI und DGE mit jeweils ca. 50\% deutlich geringer war. Bei t1 ging diese Spanne sogar noch weiter auseinander. Innerhalb KETO wurden die Sollwerte hier von fast 95\% der Patientinnen erreicht, während sich innerhalb LOGI nur sehr wenig und innerhalb DGE trotz des durchschnittlich großen Anstiegs (siehe Abbildung 74) fast gar nichts änderte.

Ähnlich wie bei VO2/kg und Last stellten sich die Ergebnisse bei der Zeit bis zum Ende der Belastung dar (siehe Abbildung 75). Die Patientinnen der KETO-Gruppe hielten die Belastung zu beiden Zeitpunkten durchschnittlich am längsten durch, während auch hier die Patientinnen der DGE-Gruppe durchschnittlich am schlechtesten abschnitten. Die Zunahme im Verlauf war allerdings innerhalb der DGE-Gruppe am größten. 


\section{Mittelwert-Plot - Zeit bis zum Ende der Belastung}

$\mathrm{p}(\mathrm{t} 0)<0,01$

$p($ Verlauf $)=0,240$

(Vertikale Balken zeigen 0,95 Konfidenzintervalle)

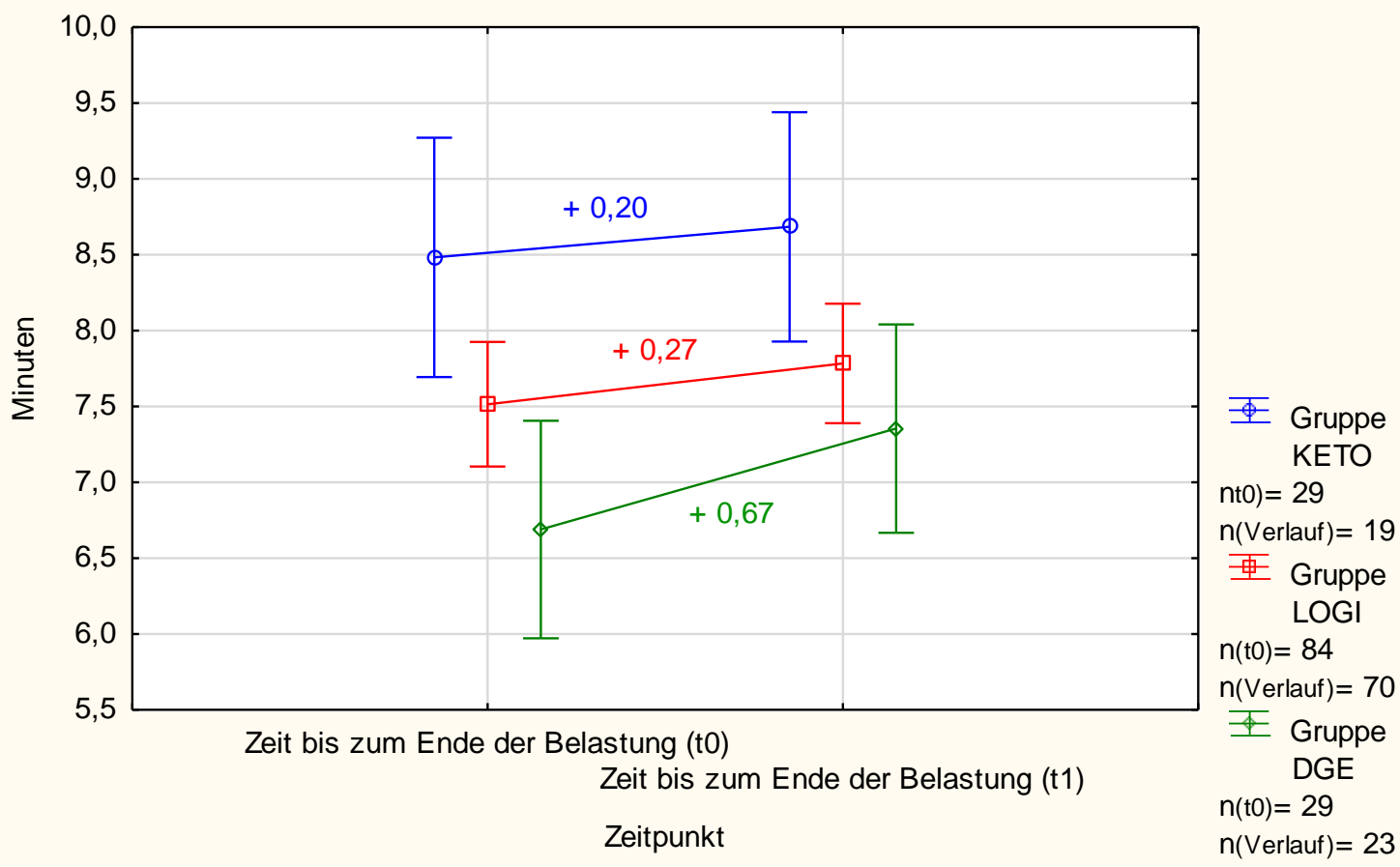

Abbildung 75: Mittelwert-Plot - Zeit bis zum Ende der Belastung

\section{Laktat 3 min. nach Belastung}

Die geringere Anzahl an Messwerten im Vergleich zu den Leistungsparametern rührt daher, dass das Messgerät zur Laktatbestimmung nicht von Anfang an zur Verfügung stand und somit bei den ersten Patientinnen aus den jeweiligen Gruppen nicht zur Anwendung kommen konnte.

Bei der Betrachtung der Ergebnisse (siehe Abbildung 76) fällt auf, dass hier ausnahmsweise nicht bei den Patientinnen der KETO-Gruppe die durchschnittlich höchsten Werte zum Zeitpunkt to gemessen wurden, sondern bei den Patientinnen der LOGIGruppe. Dafür war der durchschnittliche Anstieg innerhalb der KETO-Gruppe am größten, sodass sie bei t1 wieder die höchsten Werte erzielten. Die Patientinnen der DGEGruppe wiesen auch hier zu beiden Zeitpunkten die niedrigsten Werte auf. 


\section{Mittelwert-Plot - Laktat $3 \mathrm{~min}$. nach Belastung}

$\mathrm{p}(\mathrm{t} 0)=0,849$

$p($ Verlauf $)=0,274$

(Vertikale Balken zeigen 0,95 Konfidenzintervalle)

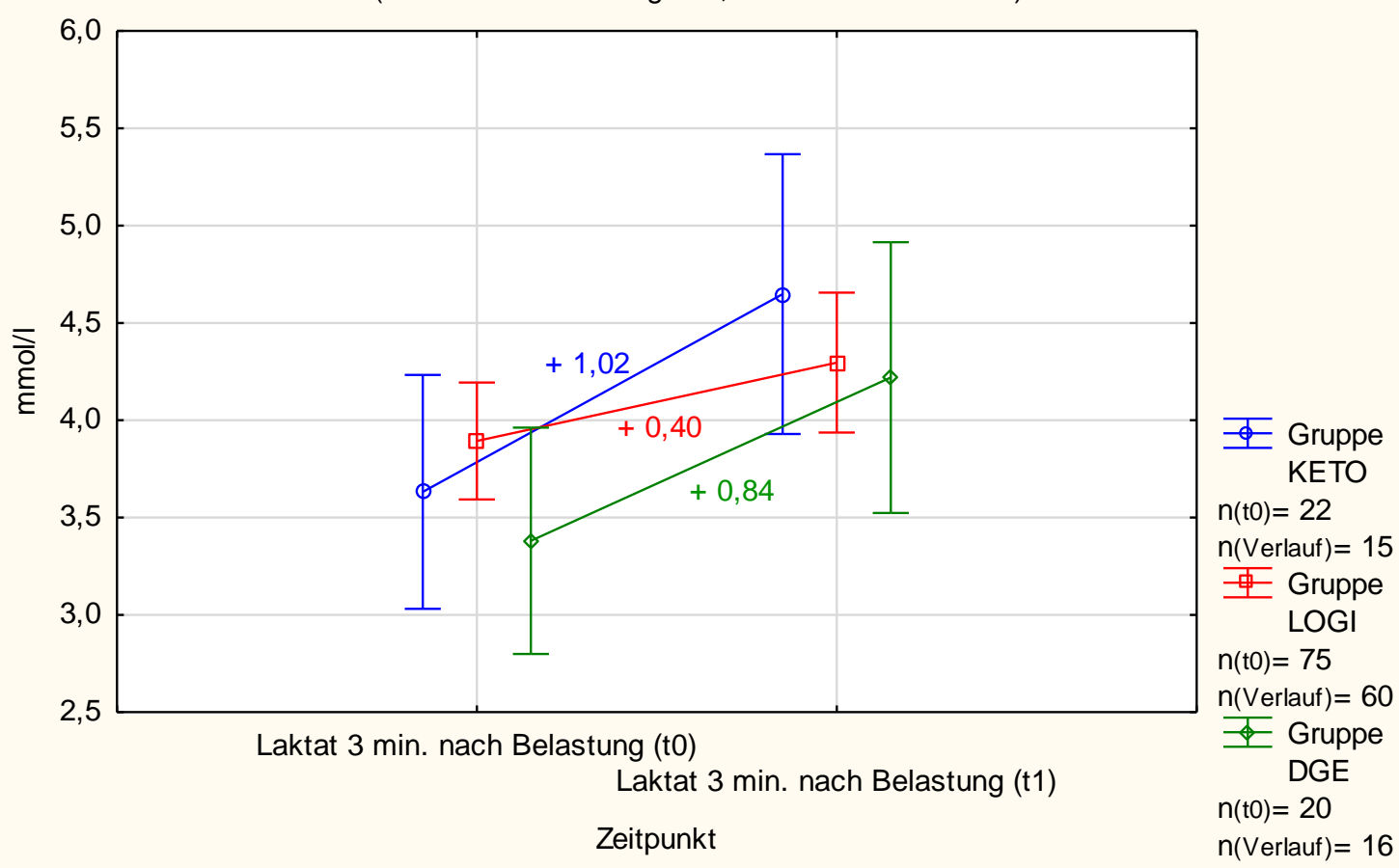

Abbildung 76: Mittelwert-Plot - Laktatmessung 3 min. nach Belastung

\section{Varianzanalysen und quantitative Veränderung im Verlauf}

Generell ist bei der Verlaufsbetrachtung wichtig zu erwähnen, dass sich bei keinem der Parameter ein signifikanter $p$-Wert für den Verlauf ergab, d. h. die in den MittelwertPlots dargestellten Veränderungen der Parameter über die Zeit unterschieden sich zwischen den Gruppen nicht signifikant voneinander.

Trotz der fehlenden Signifikanz erwähnenswert ist, dass die quantitativ größten Veränderungen innerhalb KETO beobachtet wurden, die geringsten dagegen innerhalb LOGI. Die einzelnen Veränderungen sind in Tabelle 9 noch einmal zusammengestellt. 
Tabelle 9: Durchschnittliche quantitative Veränderungen der Parameter der Spiroergometrie (t0 - t1)

\begin{tabular}{|c|c|c|c|}
\cline { 2 - 4 } \multicolumn{1}{c|}{ Verlauf t0 - t1 } & \multicolumn{2}{c|}{ Durchschnittlich größte(n) Veränderung(en) } \\
\hline Parameter & KETO & LOGI & DGE \\
\hline RER in Ruhe & $-0,469$ & $+0,005$ & $-0,004$ \\
\hline VO2/kg bei VT2 & $+2,93$ & $+0,64$ & $+0,48$ \\
\hline VO2/kg MAX & $+2,51$ & $+1,43$ & $+2,49$ \\
\hline Last bei VT2 & $+5,89$ & $-0,29$ & $-0,48$ \\
\hline Last MAX & $+4,05$ & $+3,43$ & $+7,91$ \\
\hline $\begin{array}{c}\text { Zeit bis zum Ende } \\
\text { der Belastung }\end{array}$ & $+0,20$ & $+0,27$ & $+0,67$ \\
\hline $\begin{array}{c}\text { Laktat 3 min. nach } \\
\text { Belastung }\end{array}$ & $+1,02$ & $+0,40$ & $+0,84$ \\
\hline
\end{tabular}




\section{Diskussion}

Die KOLIBRI-Studie ist die erste klinisch-kontrollierte prospektive Studie, in der die Auswirkungen verschiedener Ernährungsformen auf ein definiertes Patientenkollektiv, bestehend aus 152 Frauen mit Brustkrebs, konsekutiv über die Dauer von 20 Wochen untersucht wurden. Ziel war es, eine geeignete Ernährungsform für die TumorPatientinnen zu identifizieren, welches nicht nur zur Besserung der Lebensqualität, sondern auch zur Verbesserung der Körperzusammensetzung und der körperlichen Leistungsfähigkeit führt und somit künftig Krebspatienten als supportive Therapie eher empfohlen werden kann als andere.

Im Rahmen dieser Dissertation werden die Ergebnisse der Körperzusammensetzung und der körperlichen Leistungsfähigkeit statistisch ausgewertet und dargestellt sowie die vier in der Studie zur Erfassung der Körperzusammensetzung verwendeten Messmethoden vergleichend diskutiert. Bei diesen seit vielen Jahren anerkannten Messmethoden handelt es sich um die Dualenergie-Röntgen-Absorptiometrie (DXA), die bioelektrische Impedanzanalyse (BIA), eine handelsübliche Körperfettwaage (Fettwaage) und die Nahinfrarot-Spektroskopie (NIRS bzw. FUTREX). Jede davon ist für sich genommen anhand von Studien und dem Vergleich mit Referenzmethoden validiert, eine vergleichende Betrachtung all dieser Methoden im Rahmen einer Studie wurde bislang allerdings noch nicht durchgeführt.

Ziel hierbei ist es zum einen zu untersuchen, welche Methoden dabei gut miteinander korrelieren, d. h. vergleichbare Ergebnisse liefern, und zum anderen eine davon für zukünftige Untersuchungen als Standard-Methode in der Onkologie empfehlen zu können.

\subsection{Körperzusammensetzung - Überblick}

Obwohl der Ernährungszustand und damit auch die Körperzusammensetzung für die Prognose vieler Krebserkrankungen von entscheidender Bedeutung sind, werden er bzw. sie in der täglichen Praxis nur selten bestimmt. 
Mit jeder Messmethode lässt sich eine Vielzahl von Zielgrößen bestimmen, d. h. die Entscheidung für oder gegen eine bestimmte Methode hängt zu Beginn ganz grundsätzlich und entscheidend von den ernährungsmedizinischen Fragestellungen ab. Für die Beurteilung des Hydratationszustandes ist beispielsweise das TBW vorrangig von Bedeutung. Soll eine vorliegende Malnutrition oder drohende Sarkopenie frühzeitig diagnostiziert werden, spielt die FFM bzw. ihre Bestandteile (BCM und Muskelmasse) die entscheidende Rolle. Für das Monitoring der Therapie von Unter- oder Übergewicht sind dagegen hauptsächlich die Veränderungen der FM von Interesse (BosyWestphal et al. 2006).

Für den hier durchgeführten Methodenvergleich wurden nur diejenigen Parameter herangezogen, die von allen Methoden auch gleich ausgewiesen werden, nämlich die FM und die FFM. Trotzdem werden einige weitere methodenspezifischen Parameter und deren Bedeutung ebenfalls kurz erläutert.

Zur besseren Übersicht sind die einzelnen Zielgrößen der verschiedenen Messmethoden in Tabelle 10 noch einmal zusammengestellt.

Tabelle 10: Direkt und indirekt gemessene Zielgrößen der Messmethoden zur Erfassung der Körperzusammensetzung (modifiziert nach Bosy-Westphal et al. 2006)

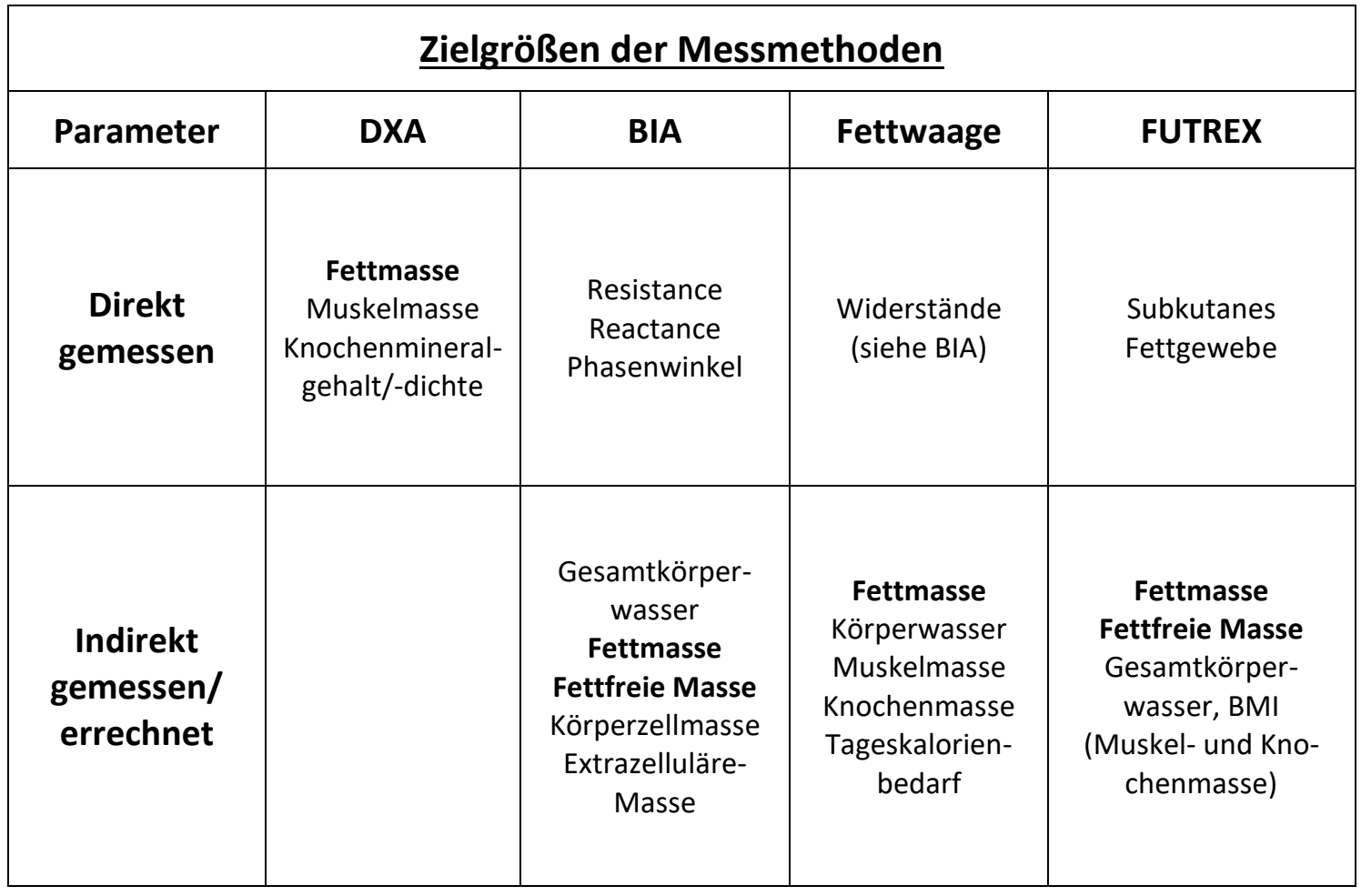




\subsection{Körperzusammensetzung - Diskussion der Ergebnisse}

\section{Studienergebnisse}

Entsprechend der Verteilung des BMI (siehe Abbildung 2) stellten sich auch die Ergebnisse für das Körpergewicht zum Zeitpunkt t0 dar. In der LOGI-Gruppe befanden sich durchschnittlich die Patientinnen mit dem größten Gewicht, gefolgt von denen der DGE-Gruppe, während die Patientinnen in der KETO-Gruppe im Durchschnitt das geringste Gewicht aufwiesen. Dieser Unterschied war zwischen KETO und LOGI mit p < 0,01 signifikant.

Unter Berücksichtigung des Studiendesigns, d. h. der fehlenden Randomisierung, der klinischen Angaben zu den Patientinnen und dem Blick auf die soziodemographischen Daten ergibt sich großer Spielraum für Annahmen, warum sich die Patientinnen letztlich bewusst für die jeweilige Ernährungsform entschieden haben könnten. Eine Erklärung dafür, warum sich so viele v. a. übergewichtige Patientinnen für die LOGI-Diät entschieden haben könnte sein, dass bekannt und beispielsweise durch Thomas et al. (2007) belegt ist, dass übergewichtige oder bereits adipöse Menschen mit Hilfe einer Diät mit niedrigerem GI mehr Gewicht verlieren können als mit Diäten mit höherem GI. Dadurch kann nicht nur das postmenopausale Erkrankungsrisiko für Brustkrebs bzw. das Mortalitätsrisiko bei bereits bestehender Brustkrebserkrankung (Mau et al. 2014), sondern durch die damit ebenfalls einhergehende Verbesserung des Lipidprofils gleichzeitig das Risiko für kardiovaskuläre Erkrankungen gesenkt werden (Heilmeyer et al. 2006). An dieser Stelle kann bereits erwähnt werden, dass die deutlichen Gewichtsunterschiede der LOGI- und DGE- gegenüber der KETO-Gruppe nicht auf die endokrine Therapie, sondern eher auf andere Lebensstilfaktoren wie eine ungesunde Ernährung und mangelnde körperliche Aktivität zurückzuführen waren. Hierfür sprechen u. a. die Ergebnisse dreier großer klinischer Studien (IBIS-I, IBIS-II und ATAC) an insgesamt über 10.000 Patienten, bei denen kein eigenständiger und unabhängiger Effekt der antihormonellen Therapie auf den Gewichtsverlauf nachgewiesen werden konnte (Sestak et al. 2012).

Ein möglicher Grund für die Wahl der ketogenen-Diät könnte gewesen sein, dass bei vielen dieser Patientinnen ein bereits metastasiertes Stadium der Erkrankung vorlag (20,7\% Metastasen gegenüber $2,2 \%$ bei LOGI bzw. $6,5 \%$ bei DGE), ihnen aufgrund des größeren Anteils an Triple-negativen Tumoren (KETO: 20,7\%, LOGI: 12\%, DGE: 6,5\%) 
weniger Therapieoptionen zur Verfügung standen und ihre Prognose dadurch deutlich schlechter war (Liedtke und Rody 2013) als bei den Patientinnen der anderen beiden Gruppen.

Daher haben sich sicherlich einige der Patientinnen bereits vorab über die schulmedizinische Therapie hinaus mit sog. komplementären Methoden wie beispielsweise der ketogenen Diät auseinandergesetzt und bereits vor Studienbeginn auch selbstständig angewendet. Unterstützt wird diese Vermutung durch die Ergebnisse der Ruheumsatzbestimmung bei der Spiroergometrie (siehe 4.5 Spiroergometrie - Diskussion der Ergebnisse). Limitierend für die langfristige Fortführung der ketogenen Diät könnten Schwierigkeiten bei der Integration in den beruflichen Alltag oder fehlender partnerschaftlicher bzw. familiärer Rückhalt gewesen sein, sodass diese beiden Aspekte vorab ebenfalls bedacht werden mussten.

Im Gegensatz zu den anderen beiden Gruppen war die Überzeugung derjenigen Patientinnen, die sich letztlich für die DGE-Ernährung entschieden haben, durch Ernährung und Bewegung eine Gewichtsreduktion herbeizuführen und dadurch ihre Erkrankung positiv beeinflussen zu können (Pinto et al. 2002) möglicherweise geringer als das Vertrauen in die altbewährte und seit Jahrzehnten als gesund geltende Ernährung wie sie von der DGE empfohlen wird. Außerdem sind die Ernährungsempfehlungen der DGE sicherlich diejenigen, die mit dem geringsten Aufwand verbunden und dementsprechend im täglichen Leben auch am einfachsten umzusetzen bzw. durchzuhalten waren. Vielleicht spielte auch eine gewisse Trägheit, geringe Selbstwirksamkeit oder die Scheu vor Veränderungen eine Rolle, oder die eben erwähnten beruflichen bzw. familiären Grundvoraussetzungen waren bei diesen Patientinnen nicht gegeben.

Die Gruppenverteilung der Fettmasse bei t0 entsprach der des Körpergewichts. Der Anteil der FM war bei der LOGI-Gruppe am größten, dicht gefolgt von der DGE-Gruppe $(p=1,0)$, wohingegen dieser innerhalb der KETO-Gruppe mit durchschnittlich 4 kg gegenüber DGE $(p=0,092)$ bzw. durchschnittlich $5 \mathrm{~kg}$ gegenüber LOGI $(p<0,01)$ deutlich geringer war.

Die Ergebnisse der FFM stellten sich im Gegensatz dazu nicht genau spiegelbildlich dar. Die Patientinnen der KETO-Gruppe wiesen nur bei der DXA-Messung die durchschnittlich größte FFM auf, bei der anderen drei Methoden lagen allerdings die Patientinnen 
der LOGI-Gruppe mit ihren Werten für die FFM durchschnittlich höher. Einheitlich dagegen war, dass die DGE die Gruppe mit der geringsten FFM darstellte.

Da im Gegensatz zu hohen Werten bei der FM niedrige Werte bei der FFM nicht automatisch auf einen schlechten Ernährungs- und Fitnesszustand hinweisen, ist für die gezielte Beurteilung von Veränderungen die Differenzierung der FFM in ihre Bestandteile BCM und ECM erforderlich.

Die ECM besteht aus der Gesamtmenge des Bindegewebes (Knochen, Knorpel, Sehnen usw.) und der extrazellulären Flüssigkeit, und die BCM beinhaltet alle stoffwechselaktiven Zellen von Muskeln und Organen und die intrazelluläre Flüssigkeit. Verluste der ECM sind meist durch Flüssigkeitsverluste (z. B. durch die Verbesserung des metabolischen Syndroms) bedingt, eine niedrige BCM weist dagegen in den meisten Fällen auf eine geringe Muskelmasse (konstitutionsbedingt oder auch durch Inaktivität oder Malnutrition) hin (Data Input 2005).

Aus diesem Grund wurden die Ergebnisse der Messungen der Muskelmasse und des Körperwassers ebenfalls ausgewertet. Aufgrund der verschiedenen Bezeichnungen der Muskelmasse (Muskelmasse bei der DXA und Fettwaage, Muskel- und Organzellmasse bei der BIA und Muskelprotein bei der NIRS) und der Tatsache, dass diese nur von der DXA, BIA und Fettwaage (Softwarefehler bei FUTREX - allerdings ohne Einfluss auf die Messwerte des BMI, der FM, FFM und des Körperwassers) und das Körperwasser nur von der BIA, Fettwaage und FUTREX (wird von der DXA generell nicht bestimmt) erhoben werden konnten, sollen an dieser Stelle nicht die absolut gemessenen Werte, sondern nur die durchschnittlichen Veränderungen von t0 - t1 in jeder Gruppe betrachtet und eine Aussage darüber getroffen werden, ob die Verluste der FFM eher durch Verluste an Muskelmasse oder Körperwasser verursacht wurden.

Wie aus Tabelle 11 und 12 hervorgeht unterschieden sich die Differenzen der Messwerte weder bei der Muskelmasse noch beim Körperwasser zwischen den drei Messmethoden innerhalb der drei Gruppen signifikant voneinander, d. h. die Methoden korrelierten bei den gemessenen Veränderungen jeweils sehr gut miteinander. Des Weiteren zeigt sich, dass die durchschnittlich gemessene Abnahme der Muskelmasse fast überall geringer war als die Abnahme des Körperwassers, sodass (unter der An- 
nahme $1 \mathrm{~kg}=1 \mathrm{I}$ ) die Abnahme der FFM zu einem größeren Anteil durch den Verlust an Körperwasser als an Muskelmasse verursacht wurde.

Tabelle 11: Durchschnittliche Differenzen bei der Muskelmasse [kg] t0 - t1 (Zur besseren Übersicht werden hier nur die Mittelwerte dargestellt, die p-Werte wurden allerdings nicht aus den Mittelwerten, sondern aus allen Messwerten innerhalb der jeweiligen Gruppe berechnet)

\begin{tabular}{|c|c|c|c|}
\cline { 2 - 4 } \multicolumn{1}{c|}{} & KETO & LOGI & DGE \\
\hline BIA & $-0,28$ & $-0,47$ & $-0,23$ \\
\hline Fettwaage & $-0,15$ & $-0,10$ & $-0,01$ \\
\hline DXA & $-0,11$ & $-0,05$ & $-0,27$ \\
\hline $\begin{array}{c}\text { Univariater Signifi- } \\
\text { kanztest }\end{array}$ & $p=0,842$ & $p=0,705$ & $p=0,807$ \\
\hline
\end{tabular}

Tabelle 12: Durchschnittliche Differenzen beim Körperwasser [I] t0 - t1 (Zur besseren Übersicht werden hier nur die Mittelwerte dargestellt, die p-Werte wurden allerdings nicht aus den Mittelwerten, sondern aus allen Messwerten innerhalb der jeweiligen Gruppe berechnet)

\begin{tabular}{|c|c|c|c|}
\cline { 2 - 4 } \multicolumn{1}{c|}{} & KETO & LOGI & DGE \\
\hline BIA & $-0,40$ & $-0,62$ & $-0,30$ \\
\hline Fettwaage & $-0,88$ & $-0,24$ & $-0,12$ \\
\hline FUTREX & $-0,46$ & $-0,77$ & $-0,18$ \\
\hline $\begin{array}{c}\text { Univariater Signifi- } \\
\text { kanztest }\end{array}$ & $p=0,677$ & $p=0,074$ & $p=0,639$ \\
\hline
\end{tabular}

Die Bestimmung der Muskelmasse ist beispielsweise deshalb relevant, um eine drohende Sarkopenie, die laut Prado et al. (2009) u. a. mit einer höheren Toxizität der Chemotherapie und einer schnelleren Tumorprogressionszeit bei metastasierten Brustkrebspatienten assoziiert ist, frühzeitig erkennen zu können. Ein steigender Körperwasseranteil kann dagegen auf eine beginnende Überwässerung hindeuten (Data Input 2005). 
Die Patientinnen hatten im Laufe der 20-wöchigen Beobachtungszeit unter allen drei Ernährungsformen an Gewicht abgenommen (siehe Abbildung 57). Im Vergleich zur KETO-Gruppe war der durchschnittliche Gewichtsverlust in der LOGI-Gruppe mehr als doppelt so groß, im Vergleich zur DGE-Gruppe sogar um mehr als das Zweieinhalbfache größer. Passend dazu ergaben die für alle drei Gruppen einzeln durchgeführten Post-hoc-Tests nur innerhalb LOGI für alle Methoden signifikante p-Werte (jeweils $p<0,01), d . h$. dass sich nur bei LOGI bei jeder Methode das zum Zeitpunkt t0 gemessene Körpergewicht signifikant von dem zum Zeitpunkt t1 gemessenen Körpergewicht unterschied.

Beinahe identisch in der Verteilung stellten sich die Ergebnisse sowohl bei der FM als auch bei der FFM dar. Bei beiden Parametern war die Abnahme innerhalb LOGI mit Abstand am größten und innerhalb DGE am geringsten. Ganz entscheidend hierbei ist die Tatsache, dass die durchschnittliche Abnahme der FM in allen drei Gruppen etwas mehr als doppelt so groß war als die Abnahme der FFM, sodass der Gewichtsverlust auch in allen drei Gruppen dementsprechend zu einem größeren Anteil auf den Verlust an FM als an FFM zurückzuführen war.

Die Patientinnen erhielten nicht nur eine Ernährungsintervention, sondern wurden während ihrer multimodalen Reha-Phase auch in allen drei Gruppen zu einer Steigerung der körperlichen Aktivität angeregt. Insofern können die hier beschriebenen Effekte nicht ausschließlich auf die Ernährungsintervention zurückgeführt werden. Es ist aber davon auszugehen, dass die Patientinnen, die eine stärkere Änderung ihres Ernährungsverhaltens befürworteten, auch zu mehr körperlicher Aktivität motiviert werden konnten, auch wenn dieser Aspekt in der KOLIBRI-Studie nicht explizit z. B. durch den Freiburger Fragebogen zur körperlichen Aktivität erfasst wurde.

In jedem Fall bleibt festzuhalten, dass Patientinnen mit Brustkrebs deutlich mehr Gewicht und FM unter einer kohlenhydratreduzierten Ernährung (LOGI) verloren hatten, als dies mit einer extrem kohlenhydratarmen (KETO) bzw. kohlenhydratreichen Ernährung (DGE) der Fall war. Damit wird die zu Beginn der Diskussion der Studienergebnisse erwähnte Aussage zu den Effekten einer kohlenhydratreduzierten Ernährung auf die Gewichtsreduktion von Patientinnen mit Adipositas und metabolischem Syndrom auch für das hier untersuchte Kollektiv bestätigt (siehe hierzu auch z. B. Shai et al. (2008) oder Westman et al. (2007)). 


\section{Weitere methodenspezifische Parameter}

Mit jeder der vier Messmethoden lassen sich weitere spezifische Parameter der Körperzusammensetzung ermitteln, sodass sich je nach zugrundeliegender Fragestellung und gewünschter Zielgrößen spezifische Vor- oder Nachteile ergeben.

Ist beispielsweise die Aufteilung bzw. Verteilung der Fettmasse im Körper von Interesse, bieten sich die DXA oder die NIRS als zu bevorzugende Messmethode an. Mittels DXA kann zum einen der Fettanteil für jede Körperregion (Kopf, obere Extremitäten, Rumpf (viszerales Fett) und untere Extremitäten) einzeln bestimmt werden (siehe Abbildung 34) und zum anderen liefert sie als einzige der vier Methoden detaillierte Informationen über die Knochenmasse (Knochenmineralgehalt und -dichte).

Im Gegensatz dazu ist durch den Einsatz einer speziellen Auswertesoftware bei der NIRS die Aufteilung der Fettmasse in essentielle Fettmasse, Fettreserve und überschüssiges Fett möglich (siehe Abbildung 48).

Mit Hilfe der BIA können zwei weitere Parameter bestimmt werden, die gezielte Aussagen über den Ernährungs- und Trainingszustand erlauben. Der Phasenwinkel gibt Hinweise auf die Zellmembranintegrität und den Gesundheitszustand des Organismus. Er sinkt beispielsweise bei kataboler Stoffwechsellage, bei Inaktivitätsatrophie oder Wassereinlagerungen und steigt entsprechend bei Beseitigung von Malnutrition oder zunehmender sportlicher Aktivität, sodass er gut als Verlaufs- bzw. Erfolgsparameter verwendet werden kann (Data Input 2005). Außerdem konnte in Studien gezeigt werden, dass der Phasenwinkel bei einigen Krankheiten wie beispielsweise HIV-Infektion oder bei allgemein fortgeschrittenem Krebsleiden sogar als Prognosemarker für den klinischen Verlauf und das Überleben herangezogen werden kann (Hui et al. 2017, Schwenk et al. 2000).

Aus den Ergebnissen der Messung des Phasenwinkels mittels BIA (siehe Abbildung 69) lässt sich eindeutig erkennen, dass die angestrebte Verbesserung um mindestens 0,5 als eines der primären Studienziele nicht erreicht werden konnte, sondern es in keiner der drei Gruppen zu signifikanten Veränderungen gekommen war. Da sich der Phasenwinkel generell nur langsam ändert, könnte der Beobachtungszeitraum der Studie von 20 Wochen möglicherweise zu kurz gewesen sein, um Veränderungen detektieren zu können. 
Der zweite BIA-spezifische Parameter, der v. a. in der Frühphase der Malnutrition entscheidend ist, ist der ECM/BCM-Index. Da bei Gesunden die BCM stets größer ist als die ECM und der Index damit kleiner 1, weist ein Anstieg entweder auf extrazelluläre Wassereinlagerungen oder den Verlust von Muskelmasse und damit auf einen schlechten Trainingszustand oder auf eine initiale Malnutrition hin (Data Input 2005).

Eine orientierende Charakterisierung des Stoffwechsels in Form des Tageskalorienbedarfs [kcal] bietet nur die Köperfettwaage. Die Berechnung erfolgt hierbei anhand von Durchschnittswerten und den Angaben zum Geschlecht, Gewicht, Größe und Aktivitätsniveau.

\subsection{Körperzusammensetzung - Grundsätze und Einschränkungen der Messmethoden}

„Die Sinnhaftigkeit des Einsatzes einer Methode zur Körperzusammensetzung richtet sich [...] [nicht nur] nach der zugrundeliegenden Fragestellung", sondern auch nach den Gütekriterien Validität und Reproduzierbarkeit sowie den zugrundeliegenden Annahmen, Erfahrungen bei bestimmten Patientenkollektiven und selbstverständlich den Kosten (Bosy-Westphal et al. 2006, S. 194). Um eine fundierte Grundlage für die Empfehlung einer der vier Messmethoden als Standardmethode zu schaffen, werden die eben genannten Aspekte anhand vorhandener Literatur nachfolgend ebenfalls vorgestellt.

\subsubsection{Validität und Reproduzierbarkeit}

Die Qualität einer Methode wird u. a. ganz entscheidend durch die Parameter Validität und Reproduzierbarkeit definiert. Unter Validität versteht man die Übereinstimmung der Messergebnisse mit denen einer Referenzmethode. Eine GoldstandardReferenzmethode für die Bestimmung der Körperzusammensetzung stellt heutzutage das 4-Kompartimentmodell (4C-Modell) dar. Wie aus der Abbildung 14 ersichtlich wird, wird der Körper mit Hilfe dieses Modells in die Kompartimente Fett (Triglyzeride), Wasser, Protein und Mineralien unterteilt. Jedes dieser Kompartimente wird hierfür getrennt mit einer eigenen Methode erfasst und so die Körperzusammensetzung insgesamt detailliert berechnet. 
Von einer guten Reproduzierbarkeit spricht man, wenn die wiederholte Messung unter gleichen Bedingungen verlässlich dieselben Messwerte liefert (Bosy-Westphal et al. 2006).

Zum Vergleich der DXA mit dem 4C-Modell sind in der Vergangenheit zahlreiche Studien durchgeführt und die entsprechenden Ergebnisse publiziert worden. In einem Review von Toombs et al. (2012) wird ein Großteil der vorhandenen Untersuchungsergebnisse verglichen und der Einfluss der enormen technischen Fortschritte der letzten 20 Jahre auf die Validität und Reproduzierbarkeit der DXA zusammengefasst. Obwohl die Mehrheit der betrachteten Studien eher eine Unterschätzung des prozentualen Körperfettanteils im Vergleich zum 4C-Modell ergab, berichten einige Studien auch von einer Überschätzung (beispielsweise Williams et al. (2006)). Diese Diskrepanz in den Messergebnissen lässt sich durch die Verwendung unterschiedlicher Gerätetypen eines Herstellers oder sogar verschiedener Hersteller erklären, was eine der wichtigsten Einschränkungen bezüglich einer verlässlichen Aussage über die Validität der DXA darstellt (Toombs et al. 2012).

Unter Berücksichtigung von acht Studien, die sich mit der Reproduzierbarkeit verschiedener DXA-Systeme auseinandergesetzt haben und von Toombs et al. (2012) vergleichend ausgewertet wurden, stellt die DXA eine sehr gut reproduzierbare Methode zur Bestimmung der Körperzusammensetzung dar. Die Ergebnisse der Variationskoeffizienten für die FM lagen im Bereich von 0,7 - 2,7\% und decken sich damit sehr gut mit denen von Bosy-Westphal et al. (2006).

Dass die DXA außerdem gut für Längsschnittuntersuchungen geeignet ist zeigen Studien, die die durchschnittlichen Veränderungen des prozentualen Körperfettanteils im Verlauf einer Gewichtsreduktion im Vergleich zum 4C-Modell verfolgten und dabei keine signifikanten Unterschiede feststellen konnten (Evans et al. 1999 oder auch Mahon et al. 2007).

Laut Pirlich et al. (2000) ist die Validität der BIA zur Bestimmung der Körperzusammensetzung bei nicht adipösen gesunden Probanden gut belegt. Diese Aussage passt u. a. zu dem bereits 1992 von Kushner publizierten Review, in dem die Ergebnisse von 12 Validierungsstudien zusammengefasst wurden, in denen die Bestimmung verschiedener Parameter wie beispielsweise des prozentualen Körperfettanteils mittels BIA mit der Deuteriumoxid-Verdünnungsmethode (D2O-TBW) und einer bestimmten Dichte- 
messung (dFFM) verglichen wurden. Der Determinationskoeffizient als Gütemaß zur Beschreibung des linearen Zusammenhangs betrug bei fast allen dieser Studien $\mathrm{R}^{2}>$ 0,90. Auch Bosy-Westphal et al. (2006) halten die BIA für eine sehr valide Methode zur Messung der Fettmasse.

Bei der BIA ist für optimale und gut reproduzierbare Ergebnisse die Einhaltung von Standarduntersuchungsbedingungen (siehe 2.2.2 Bioelektrische Impedanzanalyse) von enormer Wichtigkeit (Mally 2011). Dadurch können v. a. Störungen des Körperwasserhaushalts und daraus resultierende Schwankungen bei der Bestimmung der Körperwiderstände minimiert werden (Deutsche Gesellschaft für angewandte Sportwissenschaft 2003). Insgesamt deuten die meisten Studienergebnisse darauf hin, dass die BIA eher für längerfristige Verlaufsbeobachtungen als für die Detektion akuter Änderungen eingesetzt werden sollte (Pirlich et al. 2000).

Dass mittels leg-to-leg BIA (entspricht dem Messprinzip einer einfachen Körperfettwaage) grundsätzlich valide und im Vergleich zur konventionellen BIA bzw. anderen Bioimpedanz-basierten Methoden auch reproduzierbare Ergebnisse ermittelt werden können, postulierten sowohl Nunez et al. (1997) als auch Jebb et al. (2000). Sie hatten bei 231 bzw. 205 Erwachsenen im Alter von 18 - 79 Jahren die Körperzusammensetzung mittels leg-to-leg BIA erhoben und mit verschiedenen Referenzmethoden verglichen.

Diese Ergebnisse dürfen allerdings nicht einfach auf andere als die in den Studien untersuchten Modelle übertragen und als allgemein gültig angesehen werden. Ein ganz entscheidender Kritikpunkt, der auch auf die KOLIBRI-Studie zutrifft ist, dass der in handelsüblichen Fettwaagen befindliche Berechnungsalgorithmus (siehe 4.3.2 Zugrunde liegende Annahmen) anscheinend mehr von zusätzlichen Parametern wie Geschlecht, Körpergewicht, Körpergröße und Alter als von standardisierten Untersuchungsbedingungen abhängt. Daraus resultiert die Tatsache, dass die Verwendung solch einfacher Fettwaagen nur wenig mit einer Körperfettanalyse zu tun hat und aufgrund der extremen Abhängigkeit von den eben genannten Parametern generell eher nicht für klinische (Verlaufs-) Untersuchungen herangezogen werden sollten (Dixon et al. 2016, Tomczak 2003).

Beim Vergleich der NIRS-Messtechnik mit dem 4C-Modell geben Bosy-Westphal et al. (2006) ähnliche Werte für Validität und Reproduzierbarkeit an wie bei der BIA. Für den 
Vergleich mit anderen (Goldstandard-) Messmethoden gibt es zahlreiche Studien, die je nach Design und Durchführung zu teilweise sehr unterschiedlichen Ergebnissen bezüglich der Validität gelangten.

Dass die NIRS eine valide Methode zur Messung des Körperfettanteils im Vergleich zur Unterwasser-Fettmessung als weitere Vergleichsmethode darstellt, ergaben beispielsweise zwei von der FUTREX Inc. in Auftrag gegebene Untersuchungen an 80 bzw. 92 Personen aus den Jahren 1987 und 1996 (Davis und Paynter 1987, Dotson 1996). Im Gegensatz dazu kamen Rosenthal (1991) und Vehrs et al. (1998) in ihren Untersuchungen beim Vergleich der NIRS mit der Unterwasser-Fettmessung zu ähnlichen Ergebnissen wie Bosy-Westphal bei ihrem bereits erwähnten Vergleich der NIRS mit dem 4CModell und schätzten die NIRS aufgrund geringer Genauigkeit bzw. Unterschätzung des Körperfettanteils gegenüber der Vergleichsmethode als nicht valide genug ein.

Eine gute bis sehr gute Reproduzierbarkeit wird der NIRS allerdings übereinstimmend von den meisten Studien bescheinigt, was sicherlich $u$. a. daran liegt, dass die Durchführung der Messung grundsätzlich wenig aufwendig, gleichzeitig aber einfach und zuverlässig durchführbar ist und die wenigen potenziellen Fehlerquellen (siehe 2.2.4 Nahinfrarot-Spektroskopie) leicht eliminiert werden können.

\subsubsection{Zugrunde liegende Annahmen}

Die Validität der Messmethoden ist bei gesunden, nicht adipösen Probanden gut belegt und hängt bei allen vier Messmethoden maßgeblich von den ihnen zugrunde liegenden Annahmen ab. Die Anwendbarkeit wird durch die Grenzen der Referenzmethoden und den entsprechenden Referenzpopulationen beeinflusst, sodass die Validität aufgrund fehlender Daten bei Krebserkrankungen eingeschränkt ist.

Darüber hinaus liegt jeder Methode ein Algorithmus zugrunde, der aufgrund gewisser statistischer Beziehungen der primären Messgröße(n) auf die sekundären Zielgröße(n) schließt (Bosy-Westphal et al. 2006).

Die verschiedenen Softwareversionen und die ihnen zugrundeliegenden herstellereigenen Algorithmen bei der DXA sind nicht publiziert, sodass es wie oben bereits erwähnt teilweise zu erheblichen Schwankungen bei den Ergebnissen in der Messung der Körperzusammensetzung kommen kann, je nachdem welches Gerät von welchem Hersteller verwendet wird (Bosy-Westphal et al. 2006). Um die drei Komponenten Fettge- 
webe, Magergewebe und Knochenmineralien gleichzeitig messen zu können, müssten drei verschiedene Strahlungen mit entsprechend drei verschiedenen Energieniveaus verwendet werden (Schöllhorn 2007). Da bei der DXA-Messung allerdings nur zwei Energiemaxima zum Einsatz kommen, können auch immer nur zwei verschiedene Gewebe gleichzeitig differenziert werden (Krefting 2005), wohingegen für das fehlende dritte Kompartiment entsprechende Annahmen gemacht werden müssen (BosyWestphal et al. 2006). So kann eine inhomogene Weichteilzusammensetzung beispielsweise zu erheblichen Fehlern bei der Bestimmung der Knochenmineraldichte führen (sog. Fettfehler) (Krefting 2005).

Die Errechnung der verschiedenen Kompartimente aus den Messwerten der BIA basiert auf linearen Regressionsgleichungen, die fast ausschließlich empirisch, d. h. durch Korrelation mit Referenz-Methoden in ausgewählten Populationen gewonnen wurden (Reimers et al. 2005, Wirth 2006). Die enorme Anzahl der inzwischen publizierten Gleichungen dokumentiert laut Wirth (2006) einen der wesentlichen Kritikpunkte an der BIA. Jede der Gleichungen ist populationsspezifisch und darf somit nicht unkritisch auf andere Populationen übertragen werden.

Auf Nachfrage bezüglich des hier verwendeten BIA-Messgerätes wurde mir mitgeteilt, dass die Grundlagen der Formeln öffentlich seien, die Korrekturvariablen aber Betriebsgeheimnis der Hard- und Softwareanbieter blieben und bedacht werden müsse, dass die Validierung der BIA-Software mit Messwerten gesunder Menschen durchgeführt wurde. Hinzu kommt, dass nur wenige Gleichungen in Deutschland entwickelt wurden und „deren „Qualität“ und Akzeptanz fraglich sind“ (Bosy-Westphal et al. 2006, S. 192).

Zu den verwendeten Formeln bzw. Algorithmen der Körperfettwaagen zur Berechnung der Körperzusammensetzung sind in der Literatur keine genauen Angaben zu finden. Die meisten Hersteller gehen bei ihren Produktbeschreibungen auf diesen Aspekt gar nicht oder nur sehr oberflächlich ein. Die Geratherm Medical AG spricht in einem ihrer Internetdokumente zum Funktionsprinzip der Körperanalyse-Waagen beispielsweise davon, dass die Ergebnisse durch eine korrelierte Formel errechnet werden, „die auf der Grundlage umfassender wissenschaftlicher Analysen entwickelt wurde" (S. 2). Verweise auf diese Analysen werden allerdings nicht angegeben. Thierbach (2004) zu- 
folge schließen Körperfettwaagen aus der physikalisch gemessenen Impedanz auf die gesamte Körperflüssigkeit und durch die anschließende Division durch das Körpergewicht und Multiplikation mit der Zahl 100 erhält man schließlich den prozentualen Körperfettanteil. Auf wie viele Geräte diese einfache mathematische Formel anwendbar ist, ist nicht nachzuvollziehen. Die Überprüfung der Ergebnisse der Körperfettwaage aus der KOLIBRI-Studie ergab allerdings, dass diese die eben genannte Formel als Grundlage ihrer Berechnungen verwendete.

Der bei der NIRS (hier FUTREX) zur Berechnung des Körperfettanteils verwendete mathematische Algorithmus geht davon aus, „dass alle organischen Verbindungen Licht im nahen Infrarotbereich absorbieren und dass der Grad der Energieabsorption sich linear zur Konzentration der jeweiligen Substanzen verhält" (Schlattmann 2005, S. 5). Mit Hilfe zweier Kalibrierungskonstanten und zweiten Ableitungen der Kurven der optischen Spektren bei zwei gemessenen Wellenlängen wird der prozentuale Körperfettanteil nach Rosenthal (1991) mittels linearer Regression berechnet. Mit dieser Formel alleine lassen sich allerdings nur ungenaue Ergebnisse erzielen, die zudem schlecht mit denen von Referenzmethoden korrelieren. Um dieses Problem zu beheben und die Genauigkeit zu erhöhen ist es essentiell, die Angaben zu Geschlecht, Körpergröße und Körpergewicht zusätzlich in die Berechnung miteinzubeziehen.

\subsubsection{Erfahrungen bei Adipositas}

Aufgrund der steigenden Prävalenz von Übergewicht und Adipositas in der Bevölkerung (Bosy-Westphal et al. 2006) und des großen Anteils an übergewichtigen Patientinnen (BMI > $25 \mathrm{~kg} / \mathrm{m}^{2}$ ) in der KOLIBRI-Studie (KETO: 8 von 29; LOGI: 63 von 92; DGE: 21 von 31), sollen die bisherigen klinischen Erfahrungen und die daraus resultierenden Einschränkungen ebenfalls kurz dargestellt werden.

Die erste Hürde, an der die Messung mittels DXA bei Adipositas bereits scheitern kann ist der Untersuchungstisch bzw. dessen zulässiges Höchstgewicht (hier $160 \mathrm{~kg}$ ) und die maximale Ausdehnung des Messfeldes (hier $69 \mathrm{~cm}$ ). Bei zu großem Körperumfang können entweder nicht alle Körperregionen in die Messung eingeschlossen werden oder es kommt durch Überlappung von Gewebe zu fehlerhaften Messergebnissen bei den einzelnen Körperregionen (Brownbill und Ilich 2005). Validierungsstudien der DXA 
gegenüber GOLD-Standard-Messmethoden bei Adipositas sind relativ rar und die wenig Vorhandenen wurden an eher kleinen Patientenkollektiven durchgeführt (z. B. Bredella et al. (2010) oder LaForgia et al. (2009)). Die Tendenz der meisten Ergebnisse geht allerdings übereinstimmend in die Richtung, dass die Validität bei Adipositas nicht sehr hoch ist. Trotzdem bietet die DXA einen ganz bestimmten Vorteil gegenüber den anderen Methoden: Mittels DXA ist die Bestimmung der regionalen Fettverteilung und damit auch ganz gezielt des viszeralen Kompartiments möglich (Toombs et al. 2012), sodass zusätzliche Aussagen über ein mögliches Erkrankungsrisiko bezüglich KHK oder Diabetes mellitus Typ II getroffen werden können (Schöllhorn 2007).

Zu den wichtigsten Limitationen der BIA bei Adipositas gehört laut Deurenberg (1996) die Tatsache, dass die meisten BIA-Messgeräte bei ihren Kalkulationen von einem konstanten Hydrierungsgrad der FFM von 73,2\% ausgehen. Diese Annahme kann nicht automatisch auf Adipöse übertragen werden, da deren relativer Anteil des extrazellulären Wassers am Gesamtkörperwasser größer ist und mit steigendem BMI immer weiter zunimmt. Da auf den Rumpf fast 50\% des Körpergewichts aber nur rund $10-20 \%$ der Impedanz entfallen, wirken sich Querschnittsveränderungen des Rumpfes - wie dies v. a. bei viszeraler Adipositas der Fall ist - nur sehr geringfügig auf die Impedanz aus und es kommt zur Unterschätzung der FM bzw. Überschätzung der FFM (Deurenberg 1996, Spaniol 2004).

Deshalb und aufgrund der ausgewerteten Studien zur BIA bei Adipositas kamen die Autoren der ESPEN (The European Society of Clinical Nutrition and Metabolism) Richtlinien von 2004 zu dem Schluss, dass mittels BIA grundsätzlich valide Messergebnisse nur bis zum einem BMI von $34 \mathrm{~kg} / \mathrm{m}^{2}$ ermittelt werden können (Kyle et al. 2004).

Studien zur Validität von Fettwaagen (leg-to-leg BIA) bei Adipositas sind nicht so zahlreich vorhanden wie bei der BIA, insgesamt gelangten aber viele davon zu ähnlichen Ergebnissen. Boneva-Asiova und Boyanov (2008) kamen bei ihrem Kollektiv aus insgesamt 283 Männern und Frauen (167 davon mit BMI > $30 \mathrm{~kg} / \mathrm{m}^{2}$ ) beispielsweise zu dem Schluss, dass die leg-to-leg BIA generell gut vergleichbare Ergebnisse mit der DXA liefert, diese ab einem BMI von $>35 \mathrm{~kg} / \mathrm{m}^{2}$ v. a. aber bei Frauen mit Vorsicht betrachtet werden sollten.

Linares et al. (2011) untersuchten insgesamt 5740 aufeinander folgende Patienten ei- 
nes klinischen Ernährungsinstitutes mit durchschnittlichem BMI von $37,7 \pm 8,2 \mathrm{~kg} / \mathrm{m}^{2}$ und fanden dabei heraus, dass die leg-to-leg BIA die FM im Vergleich zur DXA im gesamten Kollektiv signifikant unterschätzte.

Somit kann auch hier geschlussfolgert werden, dass die leg-to-leg BIA bei schwerer Adipositas ebenfalls keine verlässlichen Ergebnisse liefert.

Genau wie bei den anderen drei Methoden zuvor, müssen die mittels NIRS gemessenen Ergebnisse bei Adipositas mit Vorsicht interpretiert werden und es besteht weiterer Forschungsbedarf, bevor die NIRS als alleinige Methode für die Bestimmung der Körperzusammensetzung empfohlen werden kann. Dies liegt auch hier u. a. daran, dass die Validierung inklusive der entsprechenden Berechnungsalgorithmen an gesunden, normalgewichtigen Probanden durchgeführt wurde (Panotopoulos et al. 2001).

Deshalb kommen immer wieder Studien, die die NIRS mit anderen (GOLD-) StandardMessmethoden bei adipösen Probanden verglichen haben zu dem Schluss, dass diese zwar gut miteinander korrelieren, gleichwohl immer wieder große Unterschiede in den die FM und FFM betreffenden Messergebnissen gefunden werden und diese z. T. mit steigendem BMI weiter zunehmen (z. B. Heyward et al. 1992, Jennings et al. 2010, Panotopoulos et al. 2001).

\subsubsection{Kosten}

Mit zu den wichtigsten Entscheidungskriterien für oder gegen ein Messgerät gehören sicherlich die Anschaffungskosten. Tabelle 14 gibt hierzu einen orientierenden Überblick.

Tabelle 13: Überblick über die Anschaffungskosten bzw. Preisspanne der Messgeräte

\begin{tabular}{|c|c|c|c|}
\hline DXA & BIA (Data Input) & Fettwaage & NIRS (FUTREX) \\
\hline $\begin{array}{c}40.000-50.000 € \\
\text { (je nach Hersteller } \\
\text { und Hardware- } \\
\text { /Software- } \\
\text { ausstattung) }\end{array}$ & $\begin{array}{c}\text { Ab } 3.000 € \\
\text { (inkl. Software und } \\
\text { Zubehör) }\end{array}$ & $\begin{array}{c}\mathbf{1 5}-\mathbf{2 5 0 €} \\
\text { (Geräte mit zusätzli- } \\
\text { chen Kontakten für die } \\
\text { Hände erst ab } 70 \text { - } 80 € \text {; } \\
\text { nach oben allerdings } \\
\text { keine Grenzen) }\end{array}$ & $\begin{array}{c}3.750-5.350 € \\
\text { (je nach gewünsch- } \\
\text { ter Software und } \\
\text { Zubehör) }\end{array}$ \\
\hline
\end{tabular}




\subsection{Körperzusammensetzung - Vergleich der Messmethoden}

Die Unterschiede der gemessenen durchschnittlichen Körpergewichte zum Zeitpunkt t0 rühren daher, dass das Körpergewicht bei der DXA und der Fettwaage jeweils mittels integrierter Waage gemessen wurde, für die BIA und NIRS musste das Körpergewicht dagegen standardisiert auf einer Personenwaage (siehe 2.2 Durchführung der Messungen) ermittelt werden. Es fällt auf, dass die Ergebnisse der BIA, Fettwaage und NIRS gut miteinander korrelierten, die Ergebnisse der DXA allerdings in allen drei Gruppen signifikant davon abwichen. Im Gegensatz dazu lagen die Ergebnisse der Körpergewichtsmessung zum Zeitpunkt t1 aus unbekannten Gründen näher beieinander und die signifikanten Unterschiede zwischen der DXA und den anderen Methoden war nur noch innerhalb LOGI zu finden. Dieser Unterschied lässt zwar noch keine Aussage über die Genauigkeit der Methoden zu, spielt aber sehr wahrscheinlich eine ganz entscheidende Rolle für den Methodenvergleich.

Die Betrachtung der Mittelwert-Plots für die FM und FFM zu den Zeitpunkten t0 und t1 macht deutlich, dass die Ergebnisse der Fettwaage von denen der anderen drei Methoden in erheblichem Maße abwichen.

Entsprechend ergaben die Varianzanalysen bzw. die Post-hoc-Tests bei der FM und der FFM zum Zeitpunkt t0 für alle Gruppen und bei der FM zum Zeitpunkt t1 für alle Gruppen außer KETO signifikante Unterschiede zwischen der Fettwaage und den anderen drei Methoden. In der KETO-Gruppe waren die Unterschiede bei der FM weder zwischen der Fettwaage und DXA $(p=1,0)$, noch zwischen der Fettwaage und BIA $(p=0,507)$ und auch nicht zwischen der Fettwaage und FUTREX $(p=0,103)$ signifikant. Eine Erklärung hierfür ist, dass innerhalb KETO deutlich größere Überschneidungen der 95\%-Konfidenzintervalle zwischen den vier Messmethoden vorlagen als in den anderen beiden Gruppen. Somit spricht der fehlende Unterschied nicht für die Genauigkeit der Fettwaage - zumal alle anderen Unterschiede signifikant waren.

Da weder die Varianzanalyse $(p=0,965)$ noch die Post-hoc-Tests signifikante Unterschiede für die Interaktion von "Methode*Gruppe“ bei der FFM zu Zeitpunkt t1 erbrachten, könnte man auch hier wiederum denken, dass mit der Fettwaage doch valide Messwerte erhoben werden können. Hiergegen spricht allerdings zum einen, dass beim Methodenvergleich (unabhängig von der Ernährungsform) wie bei der FM (t1), der FM (t0) und FFM (t0) auch, ein signifikanter Unterschied zwischen der Fettwaage 
und den anderen drei Methoden gefunden werden konnte und zum anderen auch wieder der Blick auf den entsprechenden Mittelwert-Plot (siehe Abbildung 56). Das extrem große 95\%-Konfidenzintervall bedeutet eine extrem große Streuung der Messwerte um den Mittelwert und spricht damit eher für die Ungenauigkeit der Messmethode - zumal die Konfidenzintervalle der anderen drei Methoden im Vergleich zur Fettwaage viel kleiner waren. Passend zu den sehr einfachen zugrundeliegenden Annahmen (siehe 4.3.2) und der Tatsache, dass die Unterschiede definitiv nicht durch ein signifikant unterschiedliches Ausgangsgewicht erklärt werden können, kann bereits an dieser Stelle festgehalten werden, dass die handelsübliche Fettwaage im Vergleich zu den anderen drei Messmethoden keine validen Messergebnisse lieferte.

Bezüglich der FM kam es zu einer signifikanten Unterschätzung, die FFM wurde dagegen entweder signifikant überschätzt oder die Messergebnisse waren aufgrund extremer Streuung sehr ungenau.

Außer den signifikanten Unterschieden zwischen der Fettwaage und den anderen drei Methoden konnten bei t0 zusätzlich auch noch signifikante Unterschiede zwischen den Ergebnissen der DXA, BIA und FUTREX gefunden werden. Bei der FM war dies nur innerhalb LOGI zwischen DXA und FUTREX der Fall, bei der FFM ebenfalls innerhalb LOGI zwischen DXA und FUTREX, zusätzlich innerhalb LOGI zwischen DXA und BIA und innerhalb DGE ebenfalls zwischen DXA und BIA. Um die drei signifikanten Unterschiede bei der FFM ein wenig zu relativieren, lohnt sich ein Blick auf die Differenzen zwischen den Mittelwerten der DXA und BIA bzw. FUTREX im Vergleich zu den Differenzen der Mittelwerte zwischen der Fettwaage und BIA bzw. FUTREX (siehe Tabelle 13).

Es fällt auf, dass die Differenzen zwischen der Fettwaage und BIA bzw. der Fettwaage und FUTREX um ein Vielfaches größer waren als diejenigen zwischen der DXA und BIA bzw. der DXA und FUTREX. Die für alle einzelnen Differenzen durchgeführten Wilcoxon-Vorzeichen-Rang-Tests ergaben in allen drei Gruppen jeweils signifikante pWerte, d. h. dass in jeder Gruppe die Differenz zwischen den Ergebnissen der Fettwaage und BIA signifikant größer war als die Differenz zwischen den Ergebnissen der DXA und BIA - entsprechendes gilt für die Differenz zwischen den Ergebnissen der Fettwaage und FUTREX im Vergleich zu den Ergebnissen der DXA und FUTREX (die genauen Kennzahlen der Verteilung sind im Anhang in Tabelle 19 aufgeführt). 
Tabelle 14: Differenzen der Mittelwerte - FFM (t0)

\begin{tabular}{|c|c|c|c|}
\hline Gruppe & Methodenvergleich & $\begin{array}{l}\text { Differenzen der } \\
\text { Mittelwerte }\end{array}$ & $\begin{array}{c}\text { Wilcoxon- } \\
\text { Vorzeichen- } \\
\text { Rang-Test }\end{array}$ \\
\hline \multirow{4}{*}{ KETO } & $D X A-B I A$ & $-0,9 \mathrm{~kg}$ & \multirow{2}{*}{$p<0,01$} \\
\hline & Fettwaage - BIA & $3,3 \mathrm{~kg}$ & \\
\hline & DXA - FUTREX & $-0,5 \mathrm{~kg}$ & \multirow{2}{*}{$p<0,01$} \\
\hline & Fettwaage - FUTREX & $3,7 \mathrm{~kg}$ & \\
\hline \multirow{4}{*}{ LOGI } & DXA - BIA & $-1,6 \mathrm{~kg}$ & \multirow{2}{*}{$p<0,01$} \\
\hline & Fettwaage - BIA & $4,6 \mathrm{~kg}$ & \\
\hline & DXA - FUTREX & $-2,0 \mathrm{~kg}$ & \multirow{2}{*}{$p<0,01$} \\
\hline & Fettwaage - FUTREX & $4,2 \mathrm{~kg}$ & \\
\hline \multirow{4}{*}{ DGE } & $D X A-B I A$ & $-1,8 \mathrm{~kg}$ & \multirow{2}{*}{$p<0,01$} \\
\hline & Fettwaage - BIA & $3,8 \mathrm{~kg}$ & \\
\hline & DXA - FUTREX & $-1,3 \mathrm{~kg}$ & \multirow{2}{*}{$p<0,01$} \\
\hline & Fettwaage - FUTREX & $4,3 \mathrm{~kg}$ & \\
\hline
\end{tabular}

Viel entscheidender ist allerdings die Tatsache, dass die zahlreichen signifikanten Unterschiede zwischen den Ergebnissen der DXA und der BIA bzw. FUTREX zum Zeitpunkt t1 weder bei der Bestimmung der FM noch bei der Bestimmung der FFM noch nachweisbar waren, sodass hier die weiter oben bereits erwähnten unterschiedlichen Ausgangsgewichte zu den beiden Zeitpunkten to und t1 berücksichtigt werden müssen. Dass sich die Ergebnisse der FM und der FFM zwischen der DXA und BIA bzw. DXA und FUTREX nur zu genau dem Zeitpunkt signifikant unterschieden, an dem auch signifikante Gewichtsunterschiede zuvor gemessen wurden, hebt die enorme Bedeutung des Ausgangsgewichts für die Genauigkeit jeder einzelnen Methode an sich und der standardisierten Gewichtserfassung als Grundlage für den nachfolgenden Methodenvergleich sehr deutlich hervor.

Aus diesem Grund - und der Tatsache, dass bei absolut gleichem Ausgangsgewicht (zu beiden Zeitpunkten) keinerlei signifikante Unterschiede zwischen den Ergebnissen der BIA und FUTREX zu finden waren - kann vermutet werden, dass bei gleichem Ausgangsgewicht aller Methoden dementsprechend auch keine signifikanten Unterschiede mehr zwischen der DXA und BIA bzw. DXA und FUTREX, sondern sehr valide und gut 
miteinander korrelierende Ergebnisse mit den drei Methoden gefunden worden wären.

Für den Methodenvergleich im zeitlichen Verlauf bzw. für die Reproduzierbarkeit der Methoden wurde untersucht, ob sich die mit jeder der vier Methoden innerhalb jeder der drei Gruppen gemessenen Veränderungen voneinander unterschieden oder nicht. Der Verlauf des Körpergewichts kann für den Methodenvergleich nicht herangezogen werden, da wie bereits erwähnt das Körpergewicht für die BIA- und FUTREX-Messung nicht mit den Geräten selbst, sondern vorab mit Hilfe einer standardisierten Personenwaage erhoben werden musste.

Anhand der Ergebnisse der Körpergewichtsmessungen lässt sich festhalten, dass die DXA, die Fettwaage und die Personenwaage gut reproduzierbare Ergebnisse lieferten und sich die durchschnittlichen Gewichtsverluste zwischen den Methoden in keiner Gruppe signifikant voneinander unterschieden. Dadurch war die Grundlage für die Beurteilung der Reproduzierbarkeit der Methoden gelegt und der Methodenvergleich für die Verlaufsmessungen möglich.

Bei der Betrachtung der Mittelwert-Plots für die FM t0 - t1 und die FFM t0 - t1 (siehe Abbildungen 63 - 65 bzw. 67 - 69) für jede einzelne Gruppe fällt zunächst die bereits erwähnte Tatsache auf, dass die Fettwaage die beiden Parameter im Vergleich zu den anderen drei Methoden signifikant unter- bzw. überschätzte.

Im Gegensatz dazu konnte bei keiner Gruppe ein signifikanter Unterschied für die durchschnittlichen Reduktionen der FM bzw. FFM zwischen den Methoden festgestellt werden. Damit lieferten alle vier Messmethoden sehr gut reproduzierbare und miteinander vergleichbare Ergebnisse über die Zeit (t0 - t1) und sind somit für Verlaufsbeobachtungen grundsätzlich gleich gut geeignet.

Wird allerdings Wert auf genaue und valide Messwerte gelegt, kann die handelsübliche Fettwaage als Messmethode nicht empfohlen werden.

Insgesamt kann unter den genannten Einschränkungen der Validität konstatiert werden, dass aufgrund der besseren Praktikabilität und Mobilität sowie der deutlich niedrigeren Kosten gegenüber der DXA sowohl die BIA als auch die NIRS als StandardMessmethode der Körperzusammensetzung für zukünftige Untersuchungen in der Onkologie empfohlen werden können. 


\subsection{Spiroergometrie - Diskussion der Ergebnisse}

Wie in der Einleitung bereits erwähnt, wirkt sich körperliche Aktivität sowohl bei präals auch postmenopausalen Frauen positiv auf das Brustkrebsrisiko aus. Da die Patientinnen der KOLIBRI-Studie alle bereits an Brustkrebs erkrankt waren ist an dieser Stelle vor allem interessant, wie sich die Ernährung auf die Fähigkeit zur körperlichen Aktivität und die Steigerung der Leistungsfähigkeit bei Brustkrebs allgemein auswirken kann.

Hinsichtlich des Benefits von körperlicher Aktivität fanden beispielsweise Holick et al. (2008) bei ihrem Kollektiv heraus, dass die Brustkrebssterblichkeit unabhängig vom Alter bei Diagnosestellung, vom Tumorstadium und vom BMI bereits durch eine Steigerung der körperlichen Aktivität auf $>2,8 \mathrm{MET}-\mathrm{h} /$ Woche $(3 \mathrm{MET}-\mathrm{h} \triangleq 1$ Stunde Gehen mit durchschnittlich $3-5 \mathrm{~km} / \mathrm{h}$ ) um $35-49 \%$ gesenkt werden konnte. 2013 stellte Steindorf auf dem Netzwerk Alternsforschung (NAR)-Seminar in Heidelberg weitere Studien vor und berichtete dabei von Ergebnissen, laut derer durch Steigerung der körperlichen Aktivität die Brustkrebssterblichkeit nach Diagnose um 34\% und das Rezidivrisiko um $24 \%$ gesenkt werden konnte.

Im Folgenden werden die Ergebnisse der körperlichen Leistungsfähigkeit des Kollektivs der KOLIBRI-Studie interpretiert und mögliche Gründe für die jeweiligen Veränderungen diskutiert.

\section{$\underline{\text { RER in Ruhe }}$}

Da die Ruheumsatzbestimmung keine Hinweise bezüglich der körperlichen Leistungsfähigkeit liefert, sondern eher Rückschlüsse auf das metabolische Substrat der Energiegewinnung zulässt, werden die Ergebnisse vorab isoliert betrachtet und bewertet. Die Ergebnisse zum Zeitpunkt t0 untermauern die Annahme, dass sich die Patientinnen bewusst für die jeweilige Ernährungsform entschieden und sich ein Großteil der Patientinnen der KETO-Gruppe bereits vorab ketogen oder zumindest fettreich ernährt hatten. Mit durchschnittlich 0,79 wiesen diese Patientinnen die niedrigsten RER-Werte auf und lagen damit in dem Bereich, in dem die Fettverbrennung die wichtigste Rolle bei der Energiegewinnung spielt. Die Patientinnen der DGE-Gruppe wiesen dagegen die höchsten Werte auf, sodass bei ihnen auch schon vor Studienbeginn der Anteil von Kohlenhydraten an der Energiegewinnung am größten war, während die Patientinnen 
der LOGI-Gruppe entsprechend der Energieverteilung ihrer Ernährungsform dazwischen lagen. Dass diese Zusammenhänge gut zutreffen zeigt außerdem die Verlaufsbetrachtung. Die RER-Werte der LOGI- und DGE-Gruppe veränderten sich kaum $(+0,005$ bzw. -0,004), sodass die Ernährungszusammensetzung in diesen beiden Gruppen zu beiden Zeitpunkten sehr ähnlich gewesen sein musste. Die Abnahme innerhalb der KETO-Gruppe weist zum einen darauf hin, dass sich nicht alle Patientinnen vorab bereits ketogen ernährt hatten, und spricht zum anderen dafür, dass Aufklärung bzw. professionelle Ernährungsberatung sowie kompetente Unterstützung für das Erreichen der Ketose offensichtlich von großer Bedeutung waren.

Generell können die Verläufe in allen drei Gruppen in der Hinsicht gewertet werden, dass sich die Mehrheit der Patientinnen, die zu beiden Zeitpunkten untersucht worden waren, an die entsprechende Vorgaben der Ernährungsform über den gesamten Zeitraum der Studie konsequent gehalten hatten.

\section{$\mathrm{VO} 2 / \mathrm{kg}$, Last und Zeit bis zum Ende der Belastung}

Zur Beurteilung der körperlichen Leistungsfähigkeit dienen die Parameter VO2/kg, Last und Zeit bis zum Ende der Belastung.

Je höher die Werte bei VT2, desto länger kann eine Belastung bei höherer Intensität bis zum Erreichen der aerob-anaeroben Schwelle durchgehalten werden. Bei der Betrachtung der entsprechenden Leistungsparameter fällt auf, dass sich innerhalb der LOGIund DGE-Gruppe jeweils nur minimale Veränderungen ergeben hatten, während die Patientinnen der KETO-Gruppe sowohl die VO2/kg bei VT2 als auch die Last bei VT2 deutlich steigern konnten.

Zur relativen maximalen Sauerstoffaufnahme (VO2/kg MAX) lässt sich vorab ganz allgemein konstatieren, dass die erzielten Messwerte v. a. bei to in allen drei Gruppen deutlich unter den von gesunden Frauen mittleren Alters durchschnittlich erreichten Werten von $30-50 \mathrm{ml} / \mathrm{min} / \mathrm{kg}$ lagen. Dies entspricht allerdings bei bestehender Brustkrebserkrankung und v. a. nach stattgehabter Chemotherapie bereits publizierten Daten (z. B. Scharhag-Rosenberger und Schommer 2013) und war somit in unseren drei Gruppen auch nicht verwunderlich. Umso erstaunlicher ist es deshalb, dass die Patientinnen der KETO-Gruppe trotz des größten Anteils an bereits metastasierten Krankheitsstadien durchschnittlich die höchsten und damit besten Werte erzielten und auch im Verlauf am meisten zulegen konnten. 
Den Mittelwert-Plots zur maximal erreichten relativen VO2 bzw. maximal erreichten Leistung in Watt (siehe Abbildung 72 und Abbildung 74) zu Folge waren die Änderungen innerhalb der DGE-Gruppe beinahe so groß bzw. sogar größer als innerhalb der KETO-Gruppe. Dies ist v. a. dem Umstand geschuldet, dass zwei Patientinnen innerhalb der DGE-Gruppe überproportional größere Leistungssteigerungen erzielen konnten als die restlichen Patientinnen der DGE-Gruppe und deren Messwerte aufgrund der relativ kleinen Anzahl an Patientinnen innerhalb der DGE-Gruppe große Auswirkungen auf den dargestellten Mittelwert hatten. Zur besseren Beurteilung und interindividuellen Vergleichbarkeit sollen deshalb an dieser Stelle die Ergebnisse der Einteilung in die Fitnesskategorien nach Cooper bzw. das Erreichen der Watt-Sollwerte nach der ACSM herangezogen und diskutiert werden (siehe Tabelle 5 - 8).

Die positiven Veränderungen bei den Fitnesskategorien waren innerhalb der KETOGruppe im Vergleich zu den anderen beiden Gruppen nicht durch das vermehrte Ausscheiden von Patientinnen aus den niedrigsten beiden Kategorien bedingt (siehe Tabelle 15), sondern kann auf die insgesamt beachtliche Leistungssteigerung der KETOGruppe zurückgeführt werden. Untermauert wird dies durch die Tatsache, dass der prozentuale Anteil an Patientinnen, die sich im Verlauf um mindestens eine Fitnesskategorie verbessern konnten, von allen drei Gruppen innerhalb der KETO-Gruppe am größten war (siehe Tabelle 16). Insgesamt waren die in Tabelle 16 aufgeführten Unterschiede allerdings nicht signifikant $(p=0,156)$.

Tabelle 15: Anteil und Zugehörigkeit zu den jeweiligen Fitnesskategorien derjenigen Patientinnen, die im Verlauf aus der Studie ausgeschieden waren

\begin{tabular}{|l|c|c|c|c|}
\hline Gruppe & $\begin{array}{c}\text { Kategorie 1 } \\
\text { und 2 }\end{array}$ & Prozent & $\begin{array}{c}\text { Kategorie 3, } \\
\text { 4 und 5 }\end{array}$ & Prozent \\
\hline KETO (n=29) & 4 & $13,79 \%$ & 5 & $17,24 \%$ \\
\hline LOGI $(n=84)$ & 9 & $10,71 \%$ & 2 & $2,38 \%$ \\
\hline DGE $(n=29)$ & 4 & $13,79 \%$ & 1 & $3,45 \%$ \\
\hline
\end{tabular}

Tabelle 16: Veränderungen der Fitnesskategorien t0 - t1

\begin{tabular}{|l|c|c|c|c|c|c|c|}
\hline Gruppe & $\begin{array}{c}\text { Anzahl Pat. } \\
\text { Gesamt }\end{array}$ & Schlechter & Prozent & Gleich & Prozent & Besser & Prozent \\
\hline KETO & 19 & 3 & $15,79 \%$ & 6 & $31,58 \%$ & 10 & $52,63 \%$ \\
\hline LOGI & 70 & 9 & $12,86 \%$ & 35 & $50 \%$ & 26 & $37,14 \%$ \\
\hline DGE & 23 & 0 & $0,00 \%$ & 15 & $65,22 \%$ & 8 & $34,78 \%$ \\
\hline
\end{tabular}


Die Ergebnisse bei den Watt-Sollwerten nach der ACSM stellten sich dementsprechend dar. Auch hier waren die positiven Veränderungen innerhalb der KETO-Gruppe nicht vorrangig durch den Wegfall von Patientinnen bedingt, die zu to den Sollwert nicht erreicht hatten und im Verlauf aus der Studie ausgeschieden waren (siehe Tabelle 17). Bei der genaueren Differenzierung der Patientinnen, deren Sollwerteinteilung sich im Verlauf nicht geändert hatte (siehe Spalte unverändert in Tabelle 18) fällt zudem auf, dass innerhalb der KETO-Gruppe hiervon alle Patientinnen bei to bereits die entsprechenden Sollwerte erreicht hatten, während dies innerhalb der LOGI- und DGE-Gruppe nur bei jeweils etwa 60\% der Fall war. Auch hier waren die in Tabelle 18 aufgeführten Unterschiede insgesamt nicht signifikant $(p=0,578)$.

Tabelle 17: Anteil derjenigen Patientinnen, die im Verlauf aus der Studie ausgeschieden waren und deren Zugehörigkeit zu den Kategorien Sollwert erreicht und Sollwert nicht erreicht

\begin{tabular}{|l|c|c|c|c|}
\hline Gruppe & $\begin{array}{c}\text { Sollwert } \\
\text { Erreicht }\end{array}$ & Prozent & $\begin{array}{c}\text { Sollwert } \\
\text { nicht erreicht }\end{array}$ & Prozent \\
\hline KETO (n=29) & 7 & $24,14 \%$ & 2 & $6,90 \%$ \\
\hline LOGI $(\mathbf{n}=\mathbf{8 4})$ & 6 & $7,14 \%$ & 5 & $5,95 \%$ \\
\hline DGE $(\mathbf{n}=\mathbf{2 9})$ & 2 & $6,90 \%$ & 3 & $10,34 \%$ \\
\hline
\end{tabular}

Tabelle 18: Veränderungen bei den Sollwerten t0 - t1

\begin{tabular}{|l|c|c|c|c|c|c|c|}
\hline Gruppe & $\begin{array}{c}\text { Anzahl Pat. } \\
\text { Gesamt }\end{array}$ & $\begin{array}{c}\text { Sollwert im Vgl. zu } \\
\text { t0 nicht mehr } \\
\text { erreicht }\end{array}$ & Prozent & unverändert & Prozent & $\begin{array}{c}\text { Sollwert im Vgl. zu } \\
\text { t0 jetzt erreicht }\end{array}$ & Prozent \\
\hline KETO & 18 & 0 & $0 \%$ & 15 & $83,33 \%$ & 3 & $16,67 \%$ \\
\hline LOGI & 61 & 4 & $6,56 \%$ & 45 & $73,77 \%$ & 12 & $19,67 \%$ \\
\hline DGE & 19 & 0 & $0 \%$ & 16 & $84,21 \%$ & 3 & $15,79 \%$ \\
\hline
\end{tabular}

Legende 2: Die geringere Anzahl an Patientinnen im Vergleich zu den Fitnesskategorien rührt daher, dass einige Patientinnen die Gewichtsgrenze von 89 kg überschritten hatten und dementsprechend für die hier vorgenommene Einteilung nicht beachtet werden konnten. Zur Spalte unverändert wurden alle Patientinnen zugeordnet, die entweder den Sollwert zu beiden Zeitpunkten nicht erreicht oder zu beiden Zeitpunkten erreicht hatten. Quantitative Zu- oder Abnahmen der erreichten Wattzahlen spielten hierbei keine Rolle.

Problematisch sowohl bei den Fitnesskategorien nach Cooper als auch bei den WattSollwerten nach der ACSM ist, dass damit nicht die gesamte Bevölkerung gleichmäßig abgebildet wird und sie somit nicht ohne weiteres auf übergewichtige und/oder an Brustkrebs erkrankte Patientinnen übertragbar sind. Außerdem besteht bei den Watt- 
Sollwerten nach der ACSM eine Altersgrenze von 64 Jahren, sodass bei älteren Patienten extrapoliert werden muss (Kroidl et al. 2010). Da allerdings keine entsprechenden Referenz- bzw. Sollwerte existieren, wurden sie unter Beachtung dieser Einschränkungen hier trotzdem als Grundlage für die Bewertung der körperlichen Leistungsfähigkeit herangezogen.

Der dritte hier bestimmte Parameter zur Beurteilung der körperlichen Leistungsfähigkeit war die Zeit bis zum Ende der Belastung. Auch hier erzielten die Patientinnen der KETO-Gruppe durchschnittlich die höchsten Werte und hielten damit von allen drei Gruppen am längsten die Belastung durch (siehe Abbildung 75).

Im Gegensatz zu den vorherigen beiden Parametern war hier allerdings die durchschnittliche Zunahme von t0 - t1 innerhalb LOGI und DGE jeweils größer als die innerhalb KETO. Ein Grund hierfür könnte gewesen sein, dass die Patientinnen der KETOGruppe mit 8,5 Minuten zum Zeitpunkt to schon sehr lange durchhielten und eine weitere Steigerung im Gegensatz zu den anderen beiden Gruppen mit deutlich niedrigeren Ausgangswerten auch deutlich schwieriger war. Nichtsdestoweniger unterschieden sich die Veränderungen von t0 - t1 zwischen den Gruppen nicht signifikant voneinander.

Die einfachste Erklärung für die insgesamt größere Zunahme der körperlichen Leistungsfähigkeit von KETO gegenüber LOGI und DGE könnte sein, dass sich die Patientinnen der KETO-Gruppe im Laufe der Studie auch insgesamt am meisten körperlich bzw. sportlich betätigt hatten. Allerdings reicht dies nicht als alleinige Begründung aus, da neben seelischem Wohlbefinden und Motivation v. a. eine ausreichende Energiebereitstellung des Körpers gewährleistet gewesen sein musste. In Anlehnung an die von Cahill (2006) veröffentlichten Erkenntnisse über den Energiestoffwechsel beim Fasten bzw. Hungern stellt sich hier die Frage, ob die aufgrund der vermehrten Lipolyse entstandenen Ketonkörper bei der ketogenen Diät von den Patientinnen der KETOGruppe nicht vielleicht sogar effizienter in Energie umgesetzt werden konnten als dies bei den anderen beiden Gruppen mit wenig bis gar keinen Ketonkörpern, aber dafür umso mehr Glukose der Fall war. In Tierversuchen mit Ratten konnten hierfür schon 
vielversprechende Ergebnisse erzielt werden, allerdings reichen diese für mehr als Spekulationen an dieser Stelle nicht aus.

Was aus den hier ausgewerteten Ergebnissen aber geschlussfolgert werden kann ist, dass die ketogene Diät den anderen beiden Ernährungsformen nicht unterlegen war, sondern eine ausgesprochen gute Ernährungsgrundlage für eine Verbesserung der körperlichen Leistungsfähigkeit trotz z. T. weit fortgeschrittener Brustkrebserkrankung darstellte.

\section{Laktat 3 min. nach Belastung}

Das Ende des aerob-anaeroben Übergangsbereiches stellt den Zeitpunkt dar, an dem ein Gleichgewicht zwischen maximaler Laktat-Eliminations- und Diffusionsrate besteht. Die Zuordnung einer fixen Laktatkonzentration erscheint zwar hilfreich, wird dem Prinzip der individuellen und dynamischen Laktatkinetik unter Belastung allerdings nicht gerecht.

Da gerade bei erkrankten Patienten diese Zuordnung erschwert ist (Westhoff et al.), wurde hier die Laktatkonzentration 3 Minuten nach Belastung ermittelt und kann somit nicht zur Beurteilung der Leistungsfähigkeit herangezogen werden, sondern dient lediglich als unterstützendes Kriterium und Anhaltspunkt, ob die aerob-anaerobe Schwelle erreicht wurde oder nicht.

Da sich weder für die einzelnen Zeitpunkte (t0 und t1) noch für die Veränderungen im Verlauf signifikante Veränderungen zwischen den Gruppen ergaben, lässt sich an dieser Stelle lediglich beschreiben, dass die Patientinnen der KETO- und LOGI-Gruppe zu beiden Zeitpunkten durchschnittlich höhere Laktatwerte erreichten als die Patientinnen der DGE-Gruppe. Dies hängt einerseits sicherlich damit zusammen, dass sich die Patientinnen der DGE-Gruppe insgesamt weniger belasteten (siehe Ergebnisse zur Zeit bis zum Ende der Belastung), andererseits bleibt trotzdem die Frage unbeantwortet, weshalb die Laktatwerte innerhalb KETO trotz gestiegenem Fettverbrennungsanteil ebenfalls angestiegen waren. Ein Erklärungsansatz ist, dass die Glykogenspeicher, die die Laktatkinetik maßgeblich beeinflussen (Klement et al. 2013), im Laufe der Studie aufgrund der basalen Energieversorgung durch Fettverbrennung doch nicht so stark aufgebraucht worden waren wie erwartet. 


\section{$5 \quad$ Zusammenfassung}

Laut Robert Koch-Institut ist Brustkrebs die häufigste Krebserkrankung bei Frauen in Deutschland. Trotz leicht gesunkener Anzahl der jährlichen Sterbefälle verursachte die Erkrankung 2012 immer noch die meisten krebsbedingten Todesfälle bei Frauen. Für verbesserte Überlebenschancen sind neben einer frühzeitigen Diagnosestellung und Fortschritten in der Therapie sowie dem zunehmenden Wissen über modifizierbare Risikofaktoren auch der Einfluss der Ernährung auf das Brustkrebsrisiko und die Prognose der Erkrankung von entscheidender Bedeutung.

Während die bisher publizierten Studienergebnisse eindeutig darauf hinweisen, dass körperliche Aktivität sowohl bei prä- als auch postmenopausalen Frauen zu einer Reduktion des Brustkrebsrisikos führt, reichen die Ergebnisse zum Thema Ernährung bei Krebs noch nicht für allgemeine Empfehlungen aus. Trotzdem haben die Beobachtungen der letzten Jahre dazu beigetragen, die über Jahrzehnte als gesund geltende Ernährung (kohlenhydratreich und fettarm), wie sie beispielsweise von der Deutschen Gesellschaft für Ernährung e.V. (DGE) empfohlen wird, bei Krebspatienten zu hinterfragen und über eine metabolisch adaptierte Ernährung nachzudenken.

Die KOLIBRI-Studie ist die erste klinisch-kontrollierte prospektive Studie, in der die Auswirkungen dreier verschiedener Ernährungsformen auf die Lebensqualität, bestimmte Laborparameter, die Körperzusammensetzung und die körperliche Leistungsfähigkeit eines definierten Patientenkollektivs, bestehend aus 152 Frauen mit Brustkrebs, konsekutiv über die Dauer von 20 Wochen untersucht wurden. Bei den drei Ernährungsformen handelt es sich um die sehr kohlenhydratarme und gleichzeitig sehr fettreiche ketogene Diät, um die kohlenhydratreduzierte LOGI-Diät und um die kohlenhydratreiche Standard-Diät nach den Empfehlungen der DGE.

Im Rahmen dieser Dissertation werden einerseits die Ergebnisse der Körperzusammensetzung und der körperlichen Leistungsfähigkeit hauptsächlich mit Hilfe von Varianzanalysen mit Messwiederholungen (ANOVA's) statistisch ausgewertet und in Mittelwert-Plots dargestellt sowie die vier in der Studie zur Erfassung der Körperzusammensetzung verwendeten Messmethoden vergleichend diskutiert. Diese sind die Dua- 
lenergie-Röntgen-Absorptiometrie (DXA), die bioelektrische Impedanzanalyse (BIA), eine handelsübliche Körperfettwaage (Fettwaage) und die Nahinfrarot-Spektroskopie (NIRS).

Im Laufe der 20-wöchigen Beobachtungszeit hatten die Patientinnen unter allen drei Ernährungsformen an Gewicht abgenommen, wobei dieser Unterschied nur innerhalb der LOGI-Gruppe signifikant war $(p<0,01)$. Die durchschnittliche Abnahme der Fettmasse (FM) war in allen drei Gruppen mehr als doppelt so groß wie die Abnahme der fettfreien Masse (FFM), sodass der Gewichtsverlust in allen drei Gruppen zu einem größeren Anteil auf den Verlust an FM als an FFM zurückzuführen war. Damit werden die Ergebnisse von Studien zu den Effekten einer kohlenhydratreduzierten Ernährung auf die Gewichtsreduktion von Patientinnen mit Adipositas und metabolischem Syndrom für das hier untersuchte Kollektiv bestätigt.

Bei der Betrachtung der körperlichen Leistungsfähigkeit, die mit Hilfe der Spiroergometrie ermittelt wurde zeigt sich, dass die Patientinnen der KETO-Gruppe trotz des größten Anteils an bereits metastasierten Brustkrebsstadien durchschnittlich die höchsten Werte erzielten und sich auch im Verlauf am meisten steigern konnten. Die größere Zunahme gegenüber der LOGI- und der DGE-Gruppe konnte allerdings nicht allein durch mehr körperliche Aktivität begründet werden, denn neben seelischem Wohlbefinden und Motivation musste eine ausreichende Energiebereitstellung des Körpers gewährleistet gewesen sein. Aus den Ergebnissen kann demnach geschlussfolgert werden, dass die ketogene Diät eine gute Ernährungsgrundlage für eine Verbesserung der körperlichen Leistungsfähigkeit trotz z. T. weit fortgeschrittener Brustkrebserkrankung war.

Für den Vergleich der Messmethoden lassen die Ergebnisse das Fazit zu, dass alle vier Messmethoden sehr gut reproduzierbare Ergebnisse lieferten und somit für Verlaufsbeobachtungen grundsätzlich gleich gut geeignet sind. Für genaue und valide Messwerte kann die handelsübliche Fettwaage als Messmethode aufgrund der signifikanten Unterschätzung der FM bzw. signifikanten Überschätzung der FFM und der z. T. aufgrund extremer Streuung sehr ungenauen Messergebnisse nicht empfohlen werden. 
Um eine fundierte Grundlage für die Empfehlung einer Standardmethode zu schaffen, wurden zusätzlich die Gütekriterien Validität und Reproduzierbarkeit sowie die zugrundeliegenden Annahmen und Erfahrungen bei bestimmten Patientenkollektiven anhand vorhandener Literatur diskutiert. Hieraus ergab sich, dass es letztlich erforderlich wäre, für jede Fragestellung bzw. Population vorab Validierungen mit krankheitsspezifischen Algorithmen an einer vergleichbaren Subpopulation durchzuführen. Da dies in der Praxis oft nicht praktikabel ist, ist die Validität der Messmethoden bei verschiedenen Erkrankungen, wie beispielweise bestimmten Krebserkrankungen oder extremer Adipositas, noch eingeschränkt.

Da bei gleichem Ausgangsgewicht zu beiden Zeitpunkten keinerlei signifikante Unterschiede zwischen den Ergebnissen der BIA und NIRS zu finden waren und aufgrund der besseren Praktikabilität und Mobilität sowie der deutlich niedrigeren Kosten im Vergleich zur DXA, können sowohl die BIA als auch die NIRS als Standard-Messmethoden für die Körperzusammensetzung für zukünftige Untersuchungen in der Onkologie empfohlen werden. 


\section{$6 \quad$ Anhang}

\section{DE}

\section{Interpretation der Werte}

\begin{tabular}{|c|c|c|c|c|c|}
\hline LCD Display & $\ldots . . .1$ & ...... imi & 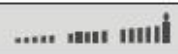 & 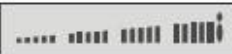 & 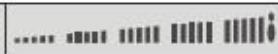 \\
\hline Bedeutung & zu dünn & dünn & normal & übergewichtig & stark übergewichtig \\
\hline
\end{tabular}

\section{Anzeigebereiche}

\begin{tabular}{|l|c|c|c|c|c|c|}
\hline & $\begin{array}{c}\text { Körper- } \\
\text { gewicht }\end{array}$ & Fett \% & Wasser \% & Muskeln \% & Knochen \% & $\begin{array}{c}\text { Kalorien- } \\
\text { bedarf }\end{array}$ \\
\hline $\begin{array}{l}\text { Mess- } \\
\text { bereich }\end{array}$ & $\begin{array}{c}150 \mathrm{~kg} \\
\sim 330 \mathrm{lb}\end{array}$ & $\begin{array}{c}4.0 \% \\
\sim 60.0 \%\end{array}$ & $\begin{array}{c}27.5 \% \\
\sim 66.0 \%\end{array}$ & $\begin{array}{c}20.0 \% \\
\sim 56.0 \%\end{array}$ & $\begin{array}{c}2.0 \% \\
\sim 20.0 \%\end{array}$ & \\
\hline Teilung & $\begin{array}{c}0.1 \mathrm{~kg} \\
0.2 \mathrm{lb}\end{array}$ & $0.1 \%$ & $0.1 \%$ & $0.1 \%$ & $0.1 \%$ & $1 \mathrm{kcal}$ \\
\hline
\end{tabular}

\section{Körperfett / Körperwasser Bewertung nach Geschlecht (W/M)}

\begin{tabular}{|c|c|c|c|c|c|c|}
\hline Alter & Fett (W) & Wasser (W) & Fett (M) & Wasser(M) & Status & Tendenzbalken \\
\hline \multirow{5}{*}{$\leqq 30$} & $4.0-16.0 \%$ & $66.0-57.8 \%$ & $4.0-11.0 \%$ & $66.0-61.2 \%$ & $\begin{array}{c}\text { zu } \\
\text { dünn }\end{array}$ & .....i \\
\hline & $16.1-20.5 \%$ & $57.7-54.7 \%$ & $11.1-15.5 \%$ & $61.1-58.1 \%$ & dūnn & أ......... \\
\hline & $20.6-25.0 \%$ & $54.6-51.6 \%$ & $15.6-20.0 \%$ & $58.0-55.0 \%$ & normal & ..... ...m nuni \\
\hline & $25.1-30.5 \%$ & $51.5-47.8 \%$ & $20.1-24.5 \%$ & $54.9-51.9 \%$ & dick & 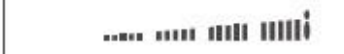 \\
\hline & $30.6-60.0 \%$ & $47.7-27.5 \%$ & $24.6-60.0 \%$ & $51.8-27.5 \%$ & $\begin{array}{l}\text { zu } \\
\text { dick }\end{array}$ & 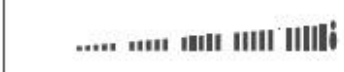 \\
\hline
\end{tabular}

\begin{tabular}{|c|c|c|c|c|c|c|}
\hline \multirow{5}{*}{$>30$} & $4.0-20.0 \%$ & $66.0-55.0 \%$ & $4.0-15.0 \%$ & $66.0-58.4 \%$ & $\begin{array}{c}\text { zu } \\
\text { dünn }\end{array}$ & .....l \\
\hline & $20.1-25.0 \%$ & $54.9-51.6 \%$ & $15.1-19.5 \%$ & $58.3-55.3 \%$ & dünn & أ...... \\
\hline & $25.1-30.0 \%$ & $51.5-48.1 \%$ & $19.6-24.0 \%$ & $55.2-52.3 \%$ & normal & ..... ..." แnni \\
\hline & $30.1-35.0 \%$ & $48.0-44.7 \%$ & $24.1-28.5 \%$ & $52.2-49.2 \%$ & dick & 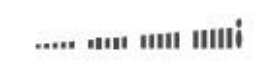 \\
\hline & $35.1-60.0 \%$ & $44.6-27.5 \%$ & $28.6-60.0 \%$ & $49.1-27.5 \%$ & $\begin{array}{l}\text { zu } \\
\text { dick }\end{array}$ & 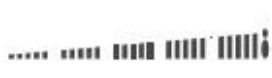 \\
\hline
\end{tabular}

Abbildung 77: : Interpretation der Tendenzbalken, Anzeigebereich der Messergebnisse und Referenzwerte zu Körperfett und Körperwasser bei der Messung der Körperzusammensetzung mittels Körperfettwaage 
Tabelle 19: Kennzahlen der Verteilung bei der FFM zum Zeitpunkt to als Grundlage für die Berechnung der Wilcoxon-Vorzeichen-Rang-Tests

\begin{tabular}{|c|c|c|c|c|c|c|}
\hline Gruppe & Methoden & Mittelwerte & Median & Unt.Quart. & Ob. Quart. & p-Wert \\
\hline \multirow{4}{*}{ KETO } & DXA-BIA & $-0,902028$ & $-0,928000$ & $-1,72310$ & 0,356700 & \multirow{2}{*}{$<0,01$} \\
\cline { 2 - 6 } & FW-BIA & 3,272041 & 3,219800 & 1,59260 & 5,240000 & \\
\cline { 2 - 6 } & DXA-FUT & $-0,520546$ & $-0,043300$ & $-2,38430$ & 0,972000 & \multirow{2}{*}{$<0,01$} \\
\cline { 2 - 6 } & FW-FUT & 3,653522 & 3,730400 & 2,99260 & 4,309000 & \\
\hline \multirow{4}{*}{ LOGI } & DXA-BIA & $-1,68688$ & $-1,64740$ & $-2,61030$ & $-0,721600$ & \multirow{2}{*}{$<0,01$} \\
\cline { 2 - 6 } & FW-BIA & 6,20463 & 6,58755 & 4,28170 & 8,256500 & \\
\cline { 2 - 6 } & DXA-FUT & $-1,99357$ & $-2,12630$ & $-3,84730$ & $-0,226300$ & \multirow{2}{*}{$<0,01$} \\
\cline { 2 - 6 } & FW-FUT & 4,22727 & 4,39790 & 3,58500 & 5,112500 & \\
\hline \multirow{4}{*}{ DGE } & DXA-BIA & $-1,74851$ & $-2,03100$ & $-2,80630$ & $-0,711100$ & \multirow{2}{*}{$<0,01$} \\
\cline { 2 - 6 } & FW-BIA & 3,80461 & 3,94755 & 2,71720 & 5,154000 & \\
\cline { 2 - 6 } & DXA-FUT & $-1,17432$ & $-1,69240$ & $-2,89840$ & 0,270300 & \multirow{2}{*}{$<0,01$} \\
\cline { 2 - 6 } & FW-FUT & 4,25794 & 4,45210 & 3,50240 & 5,011500 & \\
\hline
\end{tabular}




\section{Literaturverzeichnis}

Balady GJ, Arena R, Sietsema K, Myers J, Coke L, Fletcher GF, Forman D, Franklin B, Guazzi M, Gulati M et al. (2010): Clinician's Guide to cardiopulmonary exercise testing in adults: a scientific statement from the American Heart Association. Circulation $\underline{122}$, 191-225

Baumeister FAM: Ketogene Diät: Ernährung als Therapiestrategie: von den naturwissenschaftlichen Grundlagen zur praktischen Anwendung. SPS Publ, Heilbronn 2004 Bernau M: Untersuchungen zu einer möglichen Vorverlegung der Schlachtleistungsprüfung beim Schwein mit Hilfe der Magnetresonanztomographie (MRT) und DualenergieRöntgenabsorptiometrie (DXA) in vivo. Vet.Med.Diss. München 2011

Bertz J, Dahm S, Haberland J, Kraywinkel K, Kurth B-M, Wolf U: Verbreitung von Krebserkrankungen in Deutschland: Entwicklung der Prävalenzen zwischen 1990 und 2010. Robert-Koch-Institut, Berlin 2010

Boneva-Asiova Z, Boyanov MA (2008): Body composition analysis by leg-to-leg bioelectrical impedance and dual-energy $X$-ray absorptiometry in non-obese and obese individuals. Diabetes Obes Metab 10, 1012-1018

Borrmann F: Ernährungs- und Hydratationszustand älterer Menschen: Ein Vergleich klinischer Befunde mit Ergebnissen der Bioelektrischen Impedanzanalyse (BIA). Med.Diss. Hamburg 2013

Bosy-Westphal A, Kromeyer-Hausschild K, Pirlich M, Schlattmann A, Scholz G, Müller M (2006): Körperzusammensetzung - Was kann man wie und mit welchem Wert in der Praxis messen? Akt Ernähr Med 31, 189-195

Brandt K: Auswirkungen des Kohlenhydratanteils in der Nahrung auf den Stoffwechsel Schwangerer anhand von indirekter Kalorimetrie, bioelektrischer Impedanzanalyse und Blutzuckerueberwachung. Med.Diss. Berlin 2008

Bredella MA, Ghomi RH, Thomas BJ, Torriani M, Brick DJ, Gerweck AV, Misra M, Klibanski A, Miller KK (2010): Comparison of DXA and CT in the assessment of body composition in premenopausal women with obesity and anorexia nervosa. Obesity $\underline{18}$, 2227-2233

Breitkreutz R, Tesdal K, Jentschura D, Haas O, Leweling H, Holm E (2005): Effects of a high-fat diet on body composition in cancer patients receiving chemotherapy: a randomized controlled study. Wien Klin Wochenschr 117, 685-692 
Brennan SF, Cantwell MM, Cardwell CR, Velentzis LS, Woodside JV (2010): Dietary patterns and breast cancer risk: a systematic review and meta-analysis. Am J Clin Nutr $\underline{91}$, 1294-1302

Breuer H-WM (2004): Spiroergometrie - Vorschläge zur Standardisierung und Interpretation. Pneumologie $\underline{58}, 553-565$

Brownbill RA, Ilich JZ (2005): Measuring body composition in overweight individuals by dual energy x-ray absorptiometry. BMC Med Imaging $\underline{5}, 1-7$

Bruhn C (2012): Bedeutung von „Umweltfaktoren“ bei Mammakarzinom größer als angenommen. Dtsch Med Wochenschr 137, p5

Buckland G, Travier N, Cottet V, Gonzalez CA, Lujan-Barroso L, Agudo A, Trichopoulou A, Lagiou P, Trichopoulos D, Peeters PH et al. (2013): Adherence to the mediterranean diet and risk of breast cancer in the European prospective investigation into cancer and nutrition cohort study. Int J Cancer 132, 2918-2927

Cahill GF (2006): Fuel etabolism in starvation. Annu Rev Nutr $\underline{26}, 1-22$

Compher C, Frankenfield D, Keim N, Roth-Yousey L (2006): Best practice methods to apply to measurement of resting metabolic rate in adults: a systematic review. J Am Diet Assoc 106, 881-903

Data Input: Das B.I.A.-Kompendium. 3. Auflage; Darmstadt 2005

Dekowski L: Prospektive Studie zur periprothetischen DEXA-Messung nach Knietotalendoprothesenimplantation bei Varusgonarthrose. Med.Diss. Greifswald 2015

Deurenberg P (1996): Limitations of the bioelectrical impedance method for the assessment of body fat in severe obesity. Am J Clin Nutr $\underline{64}$, 449S-452S

Deutsche Gesellschaft für Ernährung e.V.: Begleittext zum DGE-Ernährungskreis 2014. (Von der DGE zugesendet, öffentlich nicht zugänglich).

Deutsche Krebshilfe und Deutsche Krebsgesellschaft: Der blaue Ratgeber: Ernährung bei Krebs. Bonn 2015

Dieterle P, Engling F-P, Horst H, Paul C, Robowsky K-D, Tillmann P: Die Anwendung der Nahinfrarotspektroskopie (NIRS) bei der Untersuchung von Futtermitteln und pflanzlichen Produkten. VDLUFA (Verband Deutscher Landwirtschaftlicher Untersuchungsund Forschungsanstalten) Standpunkt, Bonn 2003

Dixon JB, Bhasker AG, Lambert GW, Lakdawala M (2016): Leg to leg bioelectrical impedance analysis of percentage fat mass in obese patients - Can it tell us more than we already know? Surg Obes Relat Dis 12, 1397-1402 
Dossus L, Boutron-Ruault M-C, Kaaks R, Gram IT, Vilier A, Fervers B, Manjer J, Tjonneland A, Olsen A, Overvad K et al. (2014): Active and passive cigarette smoking and breast cancer risk: Results from the EPIC cohort. Int J Cancer 134, 1871-1888

Edlinger E (2002): Die Bedeutung der bioelektrischen Impedanzanalyse (BIA) im geriatrischen Bereich. Journal für Ernährungsmedizin, Österreich $\underline{4}$, 24-25

Emaus MJ, van Gils CH, Bakker MF, Bisschop CNS, Monninkhof EM, Bueno-de-Mesquita HB, Travier N, Berentzen TL, Overvad K, Tjonneland A et al. (2014): Weight change in middle adulthood and breast cancer risk in the EPIC-PANACEA study. Int J Cancer $\underline{135}$, 2887-2899

Emaus MJ, Peeters PHM, Bakker MF, Overvad K, Tjonneland A, Olsen A, Romieu I, Ferrari $P$, Dossus L, Boutron-Ruault MC et al. (2016): Vegetable and fruit consumption and the risk of hormone receptor-defined breast cancer in the EPIC cohort. Am J Clin Nutr $\underline{103}, 168-177$

Evans EM, Saunders MJ, Spano MA, Arngrimsson SA, Lewis RD, Cureton Kirk J. (1999): Body-composition changes with diet and exercise in obese women: a comparison of estimates from clinical methods and a 4-component model. Am J Clin Nutr $\underline{70}, 5-12$ Hanahan D, Weinberg RA (2011): Hallmarks of cancer: The Next Generation. Cell $\underline{144}$, 646-674

Haux D.: Nahinfrarotspektroskopie und funktionelle Magnet-Resonanz-Tomographie: simultaner Ansatz bei motorischer Einzelstimulation. Med.Diss. Berlin 2007

Heilmeyer P (2008): Die LOGI-Methode: Paradigmenwechsel in der Ernährungstherapie metabolischer Erkrankungen? Ernährung \& Medizin 23, 20-25

Heyward VH, Cook KL, Hicks VL, Jenkins KA, Quatrochi JA, Wilson WL (1992): Predictive Accuracy of Three Field Methods for Estimating Relative Body Fatness of Nonobese and Obese Women. Int J Sport Nutr $\underline{2}, 75-86$

Hirth C: Nichtinvasives optisches Mapping und Spektroskopie zur funktionellen Untersuchung des Gehirns: Räumliche, zeitliche und physiologische Aspekte lokaler Veränderungen der Blutoxygenierung bei funktioneller Aktivierung. Med.Diss. Berlin 1999 Holick CN, Newcomb PA, Trentham-Dietz A, Titus-Ernstoff L, Bersch AJ, Stampfer MJ, Baron JA, Egan KM, Willett WC (2008): Physical Activity and Survival after Diagnosis of Invasive Breast Cancer. Cancer Epidemiol Biomarkers Prev 17, 379-386

Hui D, Dev R, Pimental L, Park M, Cerana MA, Liu D, Bruera E (2017): Association Between Multi-frequency Phase Angle and Survival in Patients With Advanced Cancer. J Pain Symptom Manage 53, 571-577 
Jebb SA, Cole TJ, Doman D, Murgatroyd PR, Prentice AM (2000): Evaluation of the novel Tanita body-fat analyser to measure body composition by comparison with a fourcompartment model. Br J Nutr $\underline{83}, 115-122$

Jennings CL, Micklesfield LK, Lambert MI, Lambert EV, Collins M, Goedecke JH (2010): Comparison of body fatness measurements by near-infrared reactance and dualenergy X-ray absorptiometry in normal-weight and obese black and white women. $\mathrm{Br} \mathrm{J}$ Nutr $\underline{103}, 1065-1069$

Kalantar-Zadeh K, Kuwae N, Wu D, Shantouf R, Fouque D, Anker D, Block G, Kopple J (2006): Associations of body fat and its changes over time with quality of life and prospective mortality in hemodialysis patients. Am J Clin Nutr $\underline{83}, 202-210$

Kämmerer S: Entwicklung und Anwendung eines NIRS-basierten Routineverfahrens zur Analyse der relativen und absoluten Fettsäurezusammensetzung in Rind- und Schweinefleisch sowie die Untersuchung von Kandidatengenen für den bovinen Fettstoffwechsel. Naturwiss. Diss. Jena 2009

Kämmerer U, Schlatterer C, Knoll G (Hrsg.): Krebszellen lieben Zucker - Patienten brauchen Fett: Gezielt essen für mehr Kraft und Lebensqualität bei Krebserkrankungen; [Grundlagen zu Theorie und Praxis der ketogenen Ernährung]. 4. Auflage; SystemedVerlag, Lünen 2012-2013

Klement RJ, Frobel T, Albers T, Fikenzer S, Prinzhausen J, Kämmerer U (2013): A pilot case study on the impact of a self-prescribed ketogenic diet on biochemical parameters and running performance in healthy and physically active individuals. Nutrition and Medicine $1,1-27$

Knauer D: Fokalität und Frequenzabhängigkeit der vaskulären Antwort bei visueller Stimulation mit der Nahinfrarotspektroskopie. Med.Diss. Berlin 2009

Krause B-J, Beyer T, Bockisch A, Delbeke D, Kotzerke J, Minkov V, Reiser M, Willich N (2007): FDG-PET/CT in der Onkologie. Nuklearmedizin 46, 291-301

Krefting N: Einfluss von Formalinfixierung, Weichteilumgebung und Messrichtung auf Knochendichtemessungen mit der Zweienergie-Röntgen-Absorptiometrie. Med.Diss. München 2005

Kroidl RF, Schwarz S, Lehnigk B (2007): Historische Aspekte zu Belastungsuntersuchungen, speziell zur Spiroergometrie. Pneumologie $\underline{61}$, 291-294

Kroidl RF, Schwarz S, Lehnigk B, Greiwing A: Kursbuch Spiroergometrie: Technik und Befundung verständlich gemacht. 2. aktualisierte und erw. Auflage; Thieme, Stuttgart 2010 
Küpper C (2011): Die LOGI-Methode. Ernährung im Fokus 11, 488-495

Kushner RF (1992): Bioelectrical Impedance Analysis - BIA a review of principles and applications. J Am Coll Nutr $\underline{11}$ 199-209

Kyle UG, Bosaeus I, De Lorenzo AD, Deurenberg P, Elia M, Manuel Gomez J, Lilienthal Heitmann B, Kent-Smith L, Melchior J-C, Pirlich M et al. (2004): Bioelectrical impedance analysis-part II: utilization in clinical practice. Clin Nutr 23, 1430-1453

LaForgia J, Dollman J, Dale MJ, Withers RT, Hill AM (2009): Validation of DXA body composition estimates in obese men and women. Obesity $\underline{17}, 821-826$

Liedtke C, Rody A (2013): Neues zum tripelnegativen Mammakarzinom. ÄP Gynäkologie $\underline{3}, 36-39$

Linares CL, Ciangura C, Bouillot J-L, Coupaye M, Decleves X, Poitou C, Basdevant A, Oppert J-M (2011): Validity of Leg-to-Leg Bioelectrical Impedance Analysis to Estimate Body Fat in Obesity. Obes Surg 21, 917-923

Mahon AK, Flynn MG, Iglay HB, Stewart LK, Johnson CA, McFarlin BK, Campbell WW (2007): Measurement of body composition changes with weight loss in post-

menopausal women: Comparison of methods. J Nutr Health Aging 11, 203-213

Mally KC: Untersuchungen der segmentalen Bioimpedanzanalyse bei älteren Menschen unter spezieller Berücksichtigung der Aspekte Anwendbarkeit, Genauigkeit und Einflussfaktoren. Naturwissenschaftl.Diss. Kiel 2011

McDonald JA, Goyal A, Terry MB (2013): Alcohol Intake and Breast Cancer Risk: Weighing the Overall Evidence. Curr Breast Cancer Rep $\underline{5}$ Meyer T (2003): Der Respiratorische Quotient (RQ). Dtsch Z Sportmed 54, 29-30 Nunez C, Gallagher D, Visser M, Pi-Sunyer F, Wang Z, Heymsfield SB (1997): Bioimpedance analysis: evaluation of leg-to-leg system based on pressure contact foot-pad electrodes. Med Sci Sports Exerc 29, 524-531

Oehme A: Mechanisches Versagen thorakolumbaler Wirbelkörper nach Vertebro- und Kyphoplastie bei älteren Menschen - Vorhersage mittels Zwei-EnergieRöntgenabsorptiometrie (DXA) und quantitativer Computertomographie (qCT). Med.Diss. München 2013 Pandey MP, Schöggl K, Viszelj J, Widhalm K (2011): Body Composition: Dual-RöntgenAbsorptiometrie (DEXA). JEM $\underline{13}, 32-34$ 
Panotopoulos G, Ruiz JC, Guy-Grand B, Basdevant A (2001): Dual x-ray absorptiometry, bioelectrical impedance, and near infrared interactance in obese women. Medicine and Science in Sports and Exercise $\underline{33}, 665-670$

Paoli A, Grimaldi K, D'Agostino D, Cenci L, Moro T, Bianco A, Palma A (2012): Ketogenic diet does not affect strength performance in elite artistic gymnasts. J Int Soc Sports Nutr $\underline{9}, 34$

Phinney SD, Bistrian BR, Evans WJ, Gervino E, Blackburn GL (2013): The Human Metabolic Response to Chronic Ketosis Without Caloric Restriction: Preservation of Submaximal Exercise Capability with Reduced Carbohydrate Oxidation. Metabolism 32, 769776

Pietrobelli A, Formica C, Wang Z, Heymsfield SB (1996): Dual-energy X-ray absorptiometry body composition model: review of physical concepts. Am J Physiol 271, 941951

Pinto BM, Maruyama NC, Clark MM, Cruess DG, Park E, Roberts M (2002): Motivation to Modify Lifestyle Risk Behaviors in Women Treated for Breast Cancer. Mayo Clin Proc $\underline{77}, 122-129$

Pirlich M, Krüger A, Lochs H (2000): BIA-Verlaufsuntersuchungen: Grenzen und Fehlermöglichkeiten. Aktuel Ernaehr Med 25, 64-69

Prado CMM, Baracos VE, McCargar L, Reiman T, Mourtzakis M, Tonkin K, Mackey JR, Koski S, Pituskin E, Sawyer MB (2009): Sarcopenia as a Determinant of Chemotherapy Toxicity and Time to Tumor Progression in Metastatic Breast Cancer Patients Receiving Capecitabine Treatment. Clin Cancer Res 15, 2920-2926

Raschka C, Scott V, Dittmar M (2006): Ultradian comparison of different techniques for assessing body components in athletes - skinfold measurements, circumferences, near infrared interactance, and bioimpedance analysis. Med Sport 10, 58-62

Reimers C, Mersch S, Müller-Nothmann S-D (2005): Die Bioelektrische Impedanzanalyse (BIA) - Methoden zur Messung der Körperkompartimente in der Ernährungsmedizin. Schweiz Z Ganzheitsmed 17, 355-361

Reuss-Borst M: Studienprotokoll KOLIBRI-Studie 2013. Internes Protokoll, von Frau Prof. M. Reuss-Borst zugänglich gemacht, unpubliziert.

Reuss-Borst MA (2011): Ernährung und Brustkrebs - was ist Evidenz-basiert? Geburtsh Frauenheilk $\underline{71}, 1011-1013$

Robert Koch-Institut: Krebs in Deutschland 2011/2012. 10. Ausgabe; Berlin 2015 
Romieu I, Ferrari P, Rinaldi S, Slimani N, Jenab M, Olsen A, Tjonneland A, Overvad K, Boutron-Ruault M-C, Lajous M et al. (2012): Dietary glycemic index and glycemic load and breast cancer risk in the European Prospective Investigation into Cancer and Nutrition (EPIC). Am J Clin Nutr 96, 345-355

Romieu I, Scoccianti C, Chajes V, Batlle J de, Biessy C, Dossus L, Baglietto L, ClavelChapelon F, Overvad K, Olsen A et al. (2015): Alcohol intake and breast cancer in the European prospective investigation into cancer and nutrition. Int J Cancer $\underline{137}$, 19211930

Scharhag-Rosenberger F, Schommer K (2013): Die Spiroergometrie in der Sportmedizin. Dtsch Z Sportmed 64, 362-366

Schöllhorn B: Untersuchungen zur Anwendbarkeit der Dualenergie-

Röntgenabsorptiometrie (DXA) für Messung der Ganzkörperzusammensetzung bei zwei Putengenotypen. Vet.med.Diss. München 2007

Scholz AM, Baulain U (2009): Methoden zur Bestimmung der Körperzusammensetzung am lebenden Nutztier. Züchtungskunde $\underline{81}$, 86-96

Schwenk A, Beisenherz A, Römer K, Kremer G, Salzberger B, Elia M (2000): Phase angle from bioelectrical impedance analysis remains an independent predictive marker in HIV-infected patients in the era of highly active antiretroviral treatment. Am J Clin Nutr $\underline{72}, 496-501$

Sestak I, Harvie M, Howell A, Forbes JF, Dowsett M, Cuzick J (2012): Weight change associated with anastrozole and tamoxifen treatment in postmenopausal women with or at high risk of developing breast cancer. Breast Cancer Res Treat 134, 727-734

Shai I, Schwarzfuchs D, Henkin Y, Shahar DR, Witkow S, Greenberg I, Golan R, Fraser D, Bolotin A, Vardi H et al. (2008): Weight Loss with a Low-Carbohydrate, Mediterranean, or Low-Fat Diet. N Engl J Med 359, 229-241

Shukla AK, Kumar U (2006): Positron emission tomography: An overview. J Med Phys $\underline{31}, 13-21$

Spaniol U: Auswirkungen des Ernährungszustandes auf die Verträglichkeit einer Chemotherapie bei Patientinnen mit gynäkologischen Malignomen - Messungen mit der bioelektrischen Impedanzanalyse. Med. Diss. Berlin 2004

Stiedenroth N: Erhebung von Mangelernährungszuständen bei gynäkologischonkologischen Patientinnen mittels bioelektrischer Impedanzanalyse, Malnutrition Screening Tool und retrospektiver 24-Stunden Nahrungsanamnese. Med.Diss. Berlin 2009 
Stögg| T, Sperlich B (2014): Polarized training has greater impact on key endurance variables than threshold, high intensity, or high volume training. Front Physiol $\underline{5}, 1-9$ Thomas DE, Elliott EJ, Baur L (2007): Low glycaemic index or low glycaemic load diets for overweight and obesity (Review). Cochrane Database Syst Rev, CD005105

Toombs RJ, Ducher G, Shepherd JA, Souza MJ de (2012): The Impact of Recent Technological Advances on the Trueness and Precision of DXA to Assess Body Composition. Obesity $\underline{20}, 30-39$

Vehrs P, Morrow JR, Butte N (1998): Reliability and Concurrent Validity of Futrex and Bioelectrical Impedance. Int J Sports Med 19, 560-566

Voigt K: Die Unterschiede der Körperzusammensetzung bei Übergewicht in Abhängigkeit verschiedener Einflussfaktoren: Erfassung von Phasenwinkel, Resistance und Reactance sowie fettfreier Masse, Körperwasser und Fettmasse mittels Bioimpedanzanalyse bei Patienten mit BMI > 27. Med.Diss. Berlin 2009

Wally D, Velik-Salchner C (2015): Nahinfrarotspektroskopie unter kardiopulmonaler Reanimation und mechanischer Kreislaufunterstützung: Vom Operationssaal auf die Intensivstation. Med Klin Intensivmed Notfmed 110, 621-630

Westman EC, Feinmann RD, Mavropoulos JC, Vernon MC, Volek JS, Wortman JA, Yancy WS, Phinney SD (2007): Low-carbohydrate nutrition and metabolism. Am J Clin Nutr 86, 276-284

WHO: Obesity: Preventing and Managing the Global Epidemic. World Health Organization, Geneva 2000

WHO: Global Recommendations on Physical Activity for Health. World Health Organization, Geneva 2010

Williams JE, Wells JCK, Wilson CM, Haroun D, Lucas A, Fewtrell MS (2006): Evaluation of Lunar Prodigy dual-energy $\mathrm{X}$-ray absorptiometry for assessing body composition in healthy persons and patients by comparison with the criterion 4-component model. Am J Clin Nutr $\underline{83}, 1047-1054$

Wirth R (2006): Stellenwert der bioelektrischen Impedanzanalyse im Ernährungsassessment geriatrischer Patienten. Euro J Ger $\underline{8}, 96-99$

Wonisch M, Fruhwald FM, Hofmann P, Hödl R, Klein W, Kraxner W, Maier R, Pokan R, Smekal G, Watzinger N (2003): Spiroergometrie in der Kardiologie - Grundlagen der Physiologie und Terminologie. J Kardiol 10, 383-390 
Zok C (2014): Körperfettwaagen und ihr Einsatz in Sport und Medizin. Dtsch Med Wochenschr $\underline{139}, 1807$

\subsection{Internetquellen}

Becker N, Holzmeier S: Die 20 häufigsten Krebstodesursachen im Jahr 2012.

https://www.dkfz.de/de/krebsatlas/download/h20d.pdf, abgerufen am: 09.08.2016

Data Input (2015): BIA-Messung Kurzanleitung. http://www.data-

input.de/media/pdf_deutsch_2015/Data_Input_BIA-Messung_Kurzanleitung.pdf, abgerufen am: 03.05.2016

Davis PO, Paynter L (1987): Evaluation of a commercial near-infrared instrument for body composition analysis. http://www.futrex.com/wp-

content/uploads/2015/03/Evaluation-of-a-Commercial-Near-Infrared-Instrument-forBody-Composition-Analysis.pdf, abgerufen am: 08.03.2017

DCK med GmbH: Produktportfolio - FUTREX ${ }^{\circledR}$ Diagnostiksysteme. http://www.dckmed.de/produktportfolio/, abgerufen am: 28.05.2016

Deutsche Gesellschaft für angewandte Sportwissenschaft (2003): Unterscheidung zwischen der Infrarotlichtmessung (NIR) und der Bioelektrischen Impedanzanalyse (BIA). http://se75fc76ce69a0e10.jimcontent.com/download/version/1308086058/module/5 234653963/name/Studie\%20BIA\%20vs\%20NIR.pdf, abgerufen am: 08.03.2017

Deutsche Gesellschaft für Ernährung e.V. (2013): Vollwertig essen und trinken nach den 10 Regeln der DGE. https://www.dge.de/ernaehrungspraxis/vollwertigeernaehrung/10-regeln-der-dge/, abgerufen am: 27.06.2016

Deutsche Gesellschaft für Ernährung e.V. (2016a): Referenzwerte für die Nährstoffzufuhr. https://www.dge.de/wissenschaft/referenzwerte/, abgerufen am: 27.06.2016 Deutsche Gesellschaft für Ernährung e.V. (2016b): DGE-Ernährungskreis. https://www.dge.de/ernaehrungspraxis/vollwertige-ernaehrung/ernaehrungskreis/, abgerufen am: 27.06.2016

Deutsche Rentenversicherung (2009): Reha-Therapiestandards Brustkrebs: Leitlinie für die medizinische Rehabilitation der Rentenversicherung. http://www.deutscherentenversicherung.de/cae/servlet/contentblob/207070/publicationFile/32650/II_brustkrebs_downlo ad.pdf, abgerufen am: 28.11.2016 
dkfz (2011): MARIE-Studie Studienbericht. https://www.dkfz.de/de/epidemiologiekrebserkrankungen/download/MARIE_Studienbericht_2011.pdf, abgerufen am: 09.08.2016

dkfz (2013): Sport und Bewegung zur Krebsvorbeugung. https://www.krebsinformationsdienst.de/vorbeugung/risiken/sport.php, abgerufen am: 09.08.2016

dkfz (2016a): Informationsblatt: Bewegung und Sport bei Krebs. https://www.krebsinformationsdienst.de/wegweiser/iblatt/iblatt-bewegung-bei-nachkrebs.pdf, abgerufen am: 09.08.2016

dkfz (2016b): Informationsblatt: Bewegung und Sport in der Krebsvorbeugung. https://www.krebsinformationsdienst.de/wegweiser/iblatt/iblatt-bewegungkrebsvorbeugung.pdf, abgerufen am: 09.08.2016

Dotson CO (1996): Calibration of the FUTREX 6000 Body Composition Analyzers. http://www.futrex.com/wp-content/uploads/2015/03/An-Independant-Evaluation-ofthe-FUTREX-6100-by-the-University-of-Maryland.pdf, abgerufen am: 08.03.2017

Geratherm Medical AG: Die Grundlagen über die Körperzusammensetzung. http://geratherm.com/wp-content/uploads/2009/09/Grundlagen-über-dieKörperzusammensetzung-deutsch1.pdf, abgerufen am: 18.04.2016

GIT-Labor (2013): Nahinfrarotspektroskopie als schnelle, zuverlässige und kostengünstige Methode. https://www.git-labor.de/forschung/chemiephysik/nahinfrarotspektroskopie-als-schnelle-zuverlaessige-und-kostenguenstigemeth, abgerufen am: 28.05.2016

Heilmeyer P, Kohlenberg S, Dorn A, Faulhammer S, Kliebhan R (2006): Ernährungstherapie bei Diabetes mellitus Typ 2 mit kohlenhydratreduzierter Kost (LOGI-Methode). https://web.archive.org/web/20061028080046/http://www.systemed.de/bilder/Heil meyer.pdf, abgerufen am: 10.06.2016

Hochschule Luzern: Einfaktorielle Varianzanalyse mit Messwiederholung. https://www.empirical-methods.hslu.ch/entscheidbaum/unterschiede/zentraletendenz/einfaktorielle-varianzanalyse-mit-messwiederholung/, abgerufen am: 03.12.2016

Institute of Medicine (U.S.) (2011): Breast Cancer and the Environment: A Life Course Approach. https://www.nap.edu/resource/13263/BreastCancerReportbrief_2.pdf, abgerufen am: 09.08.2016 
Jones JD, Spears M (2014): Body Composition Analysis: Comparison of Bioelectrical Impedance Instruments versus Near-Infrared Instruments.

http://www.futrex.com/support/bia-vs-nir/, abgerufen am: 28.02.2017

Kämmerer U (2010): Die Ketogene Ernährung bei Krebserkrankungen: Informationen für Patienten. http://krebszellen-lieben-zucker-patienten-brauchen-fett.de/wpcontent/uploads/2012/09/InfoBroschuereKetogen1011.pdf, abgerufen am: 20.06.2016 Mau M, Kahnert S, Schaller K (2014): Adipositas und Brustkrebs. https://www.dkfz.de/de/krebspraevention/download/FzK_Adipositas_und_Brustkrebs .pdf, abgerufen am: 09.06.2016

Micro-Medical GmbH: FUTREX - Die professionellen Body Fat Analyzer. http://www.wmscdn.de/uploads/13308/13308.pdf, abgerufen am: 28.05.2016 National Cancer Institute (2009): Physical Activity and Cancer. http://www.cancer.gov/about-cancer/causes-prevention/risk/obesity/physicalactivity-fact-sheet, abgerufen am: 09.08.2016

Rosenthal RD (1991): The use of Near-IR Light to measure body fat: Presented at Scandinavian Weight Reduction Technical Conference, January 2011, Oslo. [http://www.futrex.com/wp-content/uploads/2015/03/The-Use-of-Near-IR-toMeasure-Body-Fat.pdf], abgerufen am: 29.05.2016

Schlattmann A (2005): Körperzusammensetzung: Praxis - Evidenzen - Messmethoden: Methodik der Nah-Infrarot-Messtechnik mittels Futrex. http://docplayer.org/62451425-Koerperzusammensetzung-praxis-evidenzenmessmethoden-methodik-der-nah-infrarot-messtechnik-mittels-futrex.html, abgerufen am: 28.05.2016

Schöffl V, Emmler J: Parameter Spiroergometrie. http://www.sozialstiftungbamberg.de/uploads/tx_templavoila/Parameter_Spiroergometrie_02.pdf, abgerufen am: 07.05.2016

Steindorf K (2013): Bewegung und Krebs: Die Bedeutung eines körperlich aktiven Lebensstils. NAR-Seminar, Heidelberg 2013. http://www.nar.uniheidelberg.de/md/nar/medien/pdfs/steindorf_ernaehrung_nar2013.pdf, abgerufen am: 18.04.2017

Thierbach D (2004): Wie funktioniert eine Körperfettwaage? http://www.rponline.de/panorama/wissen/wie-funktioniert-eine-koerperfettwaage-aid-1.2279896\#, abgerufen am: 26.02.2017 
Universität Zürich (2016): Einfaktorielle Varianzanalyse (mit Messwiederholung). http://www.methodenberatung.uzh.ch/de/datenanalyse/unterschiede/zentral/evaria nzmessw.html\#32, abgerufen am: 03.12.2016

Westhoff M, Lehnigk B, Rühle K-H, Greiwing A, Schomaker R, Eschenbacher H, Siepmann M: Positionspapier der AG-Spiroergometrie zu ventilatorischen und Laktatschwellen.

http://www.mesics.de/fileadmin/user/literature/Spiroergometrie/AGSpiroergometrie_Positionspapier-Schwellen.pdf, abgerufen am: 14.05.2016

Wikipedia: Lambert-beersches Gesetz. https://de.wikipedia.org/wiki/Lambertbeersches_Gesetz\#Das_Gesetz, abgerufen am: 28.05.2016

Wikipedia: Watt (Einheit). https://de.wikipedia.org/wiki/Watt_(Einheit), abgerufen am: 30.05.2016 


\section{Danksagung}

Mein Dank geht in erster Linie an Frau Prof. Dr. Monika Reuss-Borst für die Überlassung der Arbeit und die tolle Betreuung und Unterstützung bis zur Fertigstellung der Dissertation.

Des Weiteren danke ich Frau Prof. Dr. rer. hum. biol. Ulrike Kämmerer für die Betreuung und die vielen fachspezifischen Ratschläge.

Ein besonderer Dank geht an die studentischen Hilfskräfte des Instituts für Medizinische Statistik an der Georg-August-Universität Göttingen für die geduldige und ausgesprochen kompetente Unterstützung bei der statistischen Auswertung der erhobenen Daten.

Außerdem möchte ich mich bei den Mitarbeitern der Rehaklinik Am Kurpark in Bad Kissingen für die wertvolle Vorarbeit und Erhebung der Rohdaten bedanken. 